\title{
FLOW AND THERMAL FIELD MEASUREMENTS IN A COMBUSTOR SIMULATOR RELEVANT TO A GAS TURBINE AERO-ENGINE
}

\author{
Sachin S. Vakil \\ Thesis submitted to the Faculty of the \\ Virginia Polytechnic Institute and State University \\ in partial fulfillment of the requirements for the degree of
}

Master of Science

in

Mechanical Engineering

Dr. Karen A. Thole, Chair

Dr. Danesh K. Tafti

Dr. Brian Vick

November 22, 2002

Blacksburg, Virginia

Keywords: Combustor Flow and Thermal Fields, Gas Turbines, Jets in Crossflow

(C) 2002, Sachin S. Vakil 


\title{
Flow and Thermal Field Measurements in a Combustor Simulator Relevant to a Gas Turbine Aero-Engine
}

\author{
Sachin S. Vakil
}

\begin{abstract}
The highly competitive gas turbine industry has been motivated by consumer demands for higher power-to-weight ratios, increased thermal efficiencies, and reliability while maintaining affordability. In its continual quest, the industry must continually try to raise the turbine inlet temperature, which according to the well-known Brayton cycle is key to higher engine efficiencies. The desire for increased turbine inlet temperatures creates an extremely harsh environment for the combustor liner in addition to the components downstream of the combustor. Shear layers between the dilution jets and the mainstream, as well as combustor liner film-cooling interactions create a complex mean flow field within the combustor, which is not easy to model. A completely uniform temperature and velocity profile at the combustor exit is desirable from the standpoint of reducing the secondary flows in the turbine. However, this seldom occurs due to a lack of thorough mixing within the combustor. Poor mixing results in non-uniformities, such as hot streaks, and allow non-combusted fuel to exit the combustor.

This investigation developed a database documenting the thermal and flow characteristics within a combustor simulator representative of the flowfield within a gas turbine aero-engine. Three- and two-component laser Doppler velocimeter measurements were completed to quantify the flow and turbulence fields, while a thermocouple rake was used to quantify the thermal fields.

The measured results show very high turbulence levels due to the dilution flow injection. Directly downstream of the dilution jets, an increased thickness in the filmcooling was noted with a fairly non-homogeneous temperature field across the combustor width. A highly turbulent shear layer was found at the leading edge of the dilution jets. Measurements also showed that a relatively extensive recirculation region existed downstream of the dilution jets. Despite the lack of film-cooling injection at the trailing edge of the dilution hole, there existed coolant flow indicative of a horse-shoe vortex wrapping around the jet. As a result of the dilution jet interaction with the mainstream flow, kidney-shaped thermal fields and counter-rotating vortices developed. These vortices serve to enhance combustor mixing.
\end{abstract}




\section{Acknowledgments}

First, above all else, I would like to thank GOD for getting me as far as I have come in my life, and surrounding me with the best of family and friends. As long as I tried my best, I knew you would make everything turn out fine. To my advisor Dr. T, thank you for taking on a lost fellow from Virginia. You provided me with a tremendous amount of guidance and knowledge, as well as taught me to always be proud and confident of my work. Thank you for always letting me bug you with my "quick" questions and answering the countless foolish ones that I have asked in my day. I will always remember my time here at Virginia Tech and will truly miss working with you. To my family, you are my heart and soul. I love you all tremendously, thank you for always putting up with my nonsense, and pushing me to work hard and achieve as much as I can. To Dr. Tafti and Dr. Vick, thank you for your patience while I struggled to finish writing. I know that I have been telling you both I will be giving you this thesis anytime "next week". Thank you to the sponsors of my research, United Technologies and Pratt \& Whitney. To Will, Erik, Jesse, Eric, Chris, Evan, Daniel, Andy, Mike, Andrew, Lyle, and Severin. Well what can I say guys? This has been the funniest group of people I have ever worked with. Thank you for all the laughs and always making things fun around here. Big up to my KBK boys! I LOWE you guys. You have always been there and you guys will always be my bros and extended family for life. Lastly, I would like to officially put an end to this "Hokie Sac" nonsense. Come on guys, you know where the love is at. LET'S GO HOOS!

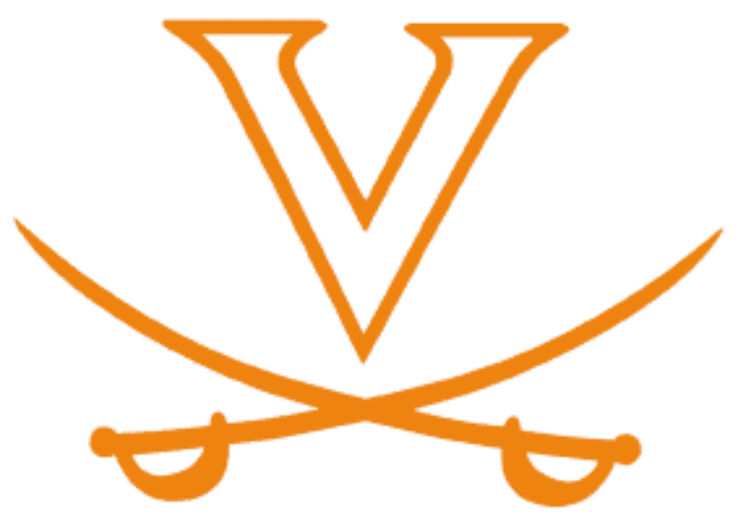




\section{Table of Contents}

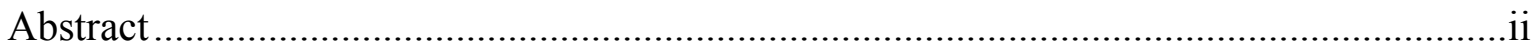

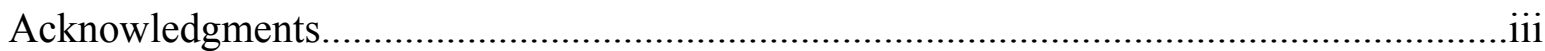

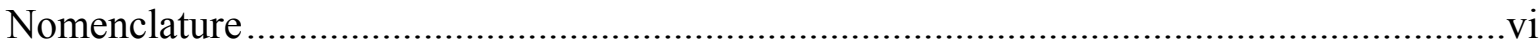

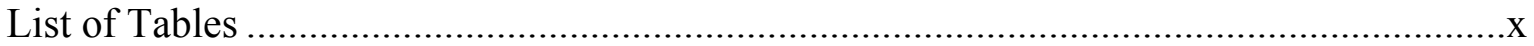

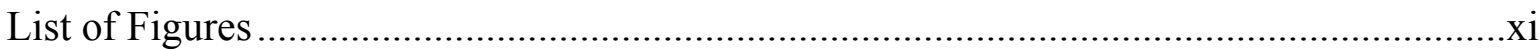

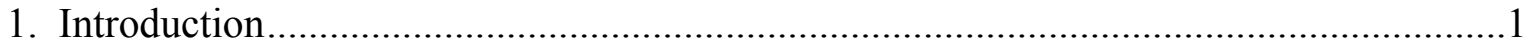

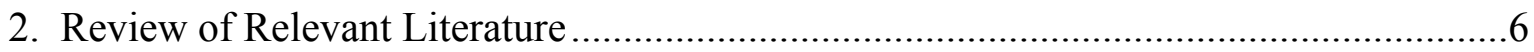

2.1 Experimental Studies and Computational Simulations for Non-Reacting Flows in Combustor Simulators ....................................................................

2.2 Reacting Combustor Experimental Studies and Computational Simulations ....12

2.3 Non-Reacting and Reacting Studies.................................................................16

2.4 Studies of Jets in Crossflow ………………………........................................17

2.5 Summary of Current Literature ……………………........................................19

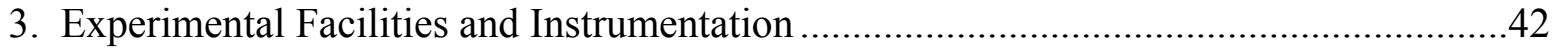

3.1 Wind Tunnel Facility.....................................................................................42

3.2 Experimental Instrumentation and Measurement Techniques ………………....51

3.3 Uncertainty Analysis ........................................................................................6

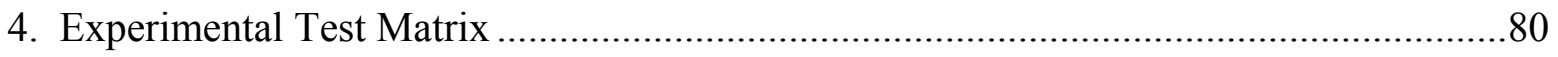

4.1 Combustor Simulator Flow and Thermal Conditions .........................................80

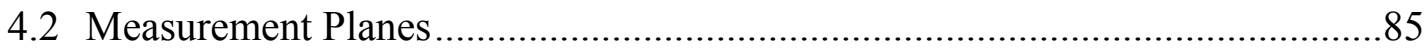

4.3 Measured Quantities and Definitions ...............................................................8

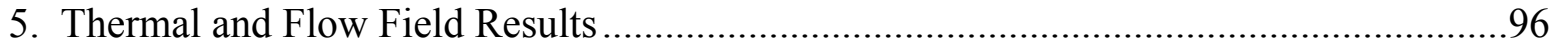

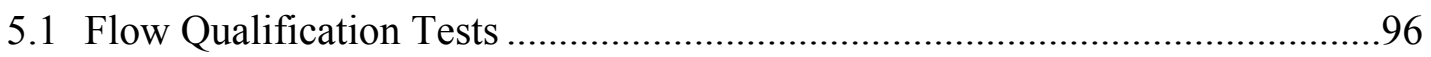

5.2 Film-Cooling Thermal Field Measurements ……………...................................98 
5.3 Thermal and Flow Field Measurements for High and Low Momentum Dilution Jets... 100

5.4 Comparisons Between Experimental and Computational Results

6. Conclusions and Recommendations for Future Work 155

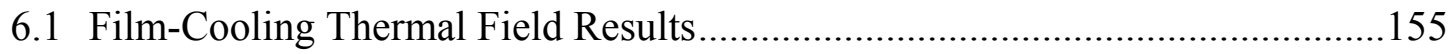

6.2 Thermal and Flow Field Results for High Momentum Dilution Jets.................156

6.3 Thermal and Flow Field Results for Low Momentum Dilution Jets .................158

6.4 Comparisons of Experimental Measurements to Computational Predictions .... 160

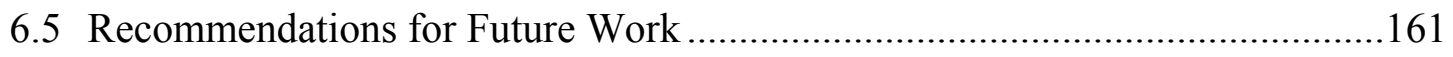

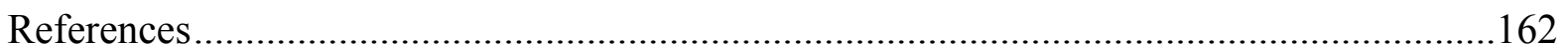

Appendix A: Characteristics of Pressure Transducers ................................................. 168

Appendix B: TSI-to-Lab Non-Orthogonal and Tilt Velocity Transformations...................179

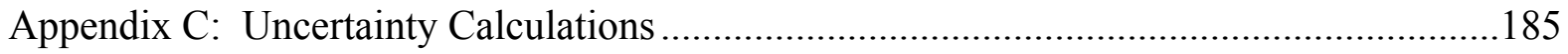

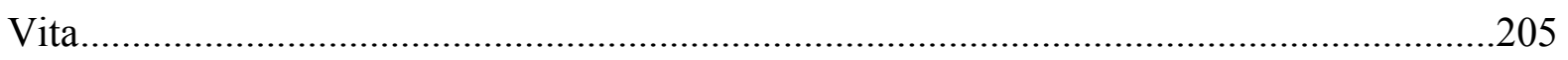




\section{Nomenclature}

$$
\begin{aligned}
& A_{c}=\quad \text { cross-sectional area of the combustor simulator } \\
& A_{\text {hole }}=\quad \text { cross-sectional area of a film-cooling or dilution hole } \\
& C_{d}=\text { discharge coefficient, } C_{d}=\frac{m_{\text {actual }}}{m_{\text {theoretical }}} \\
& d \quad=\quad \text { film-cooling hole diameter } \\
& D_{1} \quad=\quad \text { first row dilution hole diameter } \\
& D_{2} \quad=\quad \text { second row dilution hole diameter } \\
& D R=\text { density ratio, } D R=\frac{\rho_{c}}{\rho_{\infty}} \\
& H_{\text {in }} \quad=\quad \text { combustor inlet height } \\
& I=\text { momentum flux ratio, } I=\frac{\rho_{j e t} \cdot u_{j e t}^{2}}{\rho_{\infty} \cdot u_{\infty}^{2}} \\
& \text { ID } \quad=\quad \text { inner diameter } \\
& k=\quad \text { turbulent kinetic energy, } k=\frac{1}{2}\left(u_{r m s}^{2}+v_{r m s}^{2}+w_{r m s}^{2}\right) \text { for three-component } \\
& \text { flowfields, and } k=\frac{1}{2}\left(u_{r m s}^{2}+w_{r m s}^{2}\right) \text { for tow-component flowfields. } \\
& K=\text { acceleration parameter, } K=\frac{v}{u_{\infty}^{2}} \frac{d u_{\infty}}{d x} \\
& L \quad=\quad \text { combustor length } \\
& \dot{m} \quad=\quad \text { mass flow rate, } \dot{m}=\rho \cdot u \cdot A \\
& M \quad=\quad \text { mass flux ratio, } M=\frac{\rho_{j e t} \cdot u_{j e t}}{\rho_{\infty} \cdot u_{\infty}} \\
& n \quad=\quad \text { total number of velocity samples } \\
& N=\text { number of film-cooling or dilution holes within a panel or row } \\
& O D=\text { outer diameter } \\
& P_{W y e}=\quad \text { maximum power for a circuit wired in the Wye configuation, } P_{W y e}=\frac{V_{L}^{2}}{R}
\end{aligned}
$$


$P_{\text {Delta }}=\quad$ maximum power for a circuit in the Delta configuration, $P_{\text {Delta }}=\frac{3 V_{L}^{2}}{R}$

$P F \quad=\quad$ pattern factor, $P F=\frac{T_{\text {max }}-T_{\text {exit }}}{T_{\text {exit }}-T_{\text {inlet }}}$

$R \quad=\quad$ resistance of a single leg in the Delta or Wye configurations

$S_{p} \quad=\quad$ pitchwise film-cooling hole spacing

$S_{S} \quad=\quad$ streamwise film-cooling hole spacing

$T=$ local temperature

$T_{c}=$ coolant temperature

$T_{\text {exit }}=$ averaged combustor exit temperature

$T_{\infty}=$ freestream temperature

$T_{\text {inlet }}=$ averaged combustor inlet temperature

$T_{\max }=$ maximum spatial combustor exit temperature

$T L=$ turbulence level, $T L=\frac{\sqrt{\frac{1}{3}\left(u_{r m s}^{2}+v_{r m s}^{2}+w_{r m s}^{2}\right)}}{u_{i n}}$ for three-component

flowfields and $T L=\frac{\sqrt{\frac{1}{2}\left(u_{r m s}^{2}+w_{r m s}^{2}\right)}}{u_{i n}}$ for two-component measurements.

$T u=$ local turbulence level, $T u=\frac{\sqrt{\frac{1}{3}\left(u_{r m s}^{2}+v_{r m s}^{2}+w_{r m s}^{2}\right)}}{\sqrt{u^{2}+v^{2}+w^{2}}}$ for three-component flowfields and $T u=\frac{\sqrt{\frac{1}{2}\left(u_{r m s}^{2}+w_{r m s}^{2}\right)}}{\sqrt{u^{2}+w^{2}}}$ for two-component measurements.

$T u_{a i}=$ "absolute intensity" defined by Van Fossen et al. (2002), $T u_{a i}=\frac{\sqrt{\rho u_{r m s}^{2}}+\overline{\rho v_{r m s}^{2}}}{\rho u}$

$u, \bar{u}=$ mean local streamwise velocity component, $\bar{u}=\left(\frac{\sum_{i=1}^{n} u_{i} \cdot \tau_{i}}{\sum_{i=1}^{n} \tau_{i}}\right)$ 


$$
\begin{aligned}
& u_{b} \quad=\quad \text { mean measured velocity by the blue laser beam pair crossing } \\
& u_{g} \quad=\quad \text { mean measured velocity by the green laser beam pair crossing } \\
& u_{\text {in }}=\text { combustor inlet velocity } \\
& u_{\infty}=\text { local freestream } / \text { mainstream velocity } \\
& u_{\text {jet }}=\text { actual velocity of a film-cooling or dilution jet } \\
& u_{\text {jet, ideal }}=\quad \text { ideal velocity of a film-cooling or dilution jet } \\
& u^{\prime}=\text { non-tilt corrected streamwise velocity } \\
& u_{r m s}=\quad \text { root-mean-square of local streamwise velocity, } u_{r m s}=\sqrt{\left(\frac{\sum_{i=1}^{n} u_{i}^{2} \cdot \tau_{i}}{\sum_{i=1}^{n} \tau_{i}}-\bar{u}^{2}\right)} \\
& u_{v} \quad=\quad \text { mean measured velocity by the violet laser beam pair crossing } \\
& v=\text { mean local pitchwise velocity component } \\
& v^{\prime}=\text { non-tilt corrected pitchwise velocity } \\
& v_{r m s}=\text { root-mean-square of local pitchwise velocity } \\
& V_{D C}=\quad \mathrm{DC} \text { voltage } \\
& V_{L} \quad=\quad \text { line voltage } \\
& V R=\quad \text { velocity ratio, } V R=\frac{u_{j e t}}{u_{\infty}} \\
& w \quad=\quad \text { mean local spanwise (vertical) velocity component } \\
& w^{\prime}=\text { non-tilt corrected spanwise velocity } \\
& w_{r m s}=\text { root-mean-square of local pitchwise velocity } \\
& W \quad=\quad \text { one sector width (two vane pitches) } \\
& X=\text { streamwise direction } \\
& Y=\text { pitchwise direction } \\
& Z=\text { spanwise (vertical) direction }
\end{aligned}
$$




\section{Greek}

$$
\begin{aligned}
& \Delta P=\text { total-to-static pressure difference, } \Delta P=\frac{I \cdot u_{\infty}^{2} \cdot \rho_{\infty}}{2 \cdot C_{d}^{2}} \text { (panel total-to-static) or } \\
& \Delta P=\frac{u_{j e t}^{2} \cdot \rho_{j e t}}{2}(\text { pitot-tube) } \\
& v \quad=\quad \text { kinematic viscosity } \\
& \rho_{c} \quad=\quad \text { coolant density } \\
& \rho_{\text {jet }} \quad=\quad \text { jet density } \\
& \rho_{\infty} \quad=\quad \text { freestream } / \text { mainstream density } \\
& \tau=\text { residence time of a particle in the probe volume created by the laser beam } \\
& \text { pair crossing } \\
& \theta \quad=\quad \text { non-dimensional temperature, } \theta=\frac{T_{\infty}-T}{T_{\infty}-T_{c}} \\
& \theta_{g}=\quad \text { probe angle off the } Y \text {-axis applied to the two-component (blue and green) } \\
& \text { LDV probe } \\
& \theta_{\text {tilt }}=\quad \text { tilt angle applied to LDV probe } \\
& \theta_{v}=\text { probe angle off the } Y \text {-axis applied to the single-component (violet) LDV } \\
& \text { probe }
\end{aligned}
$$




\section{List of Tables}

Table 3.1 Typical Tunnel Operating Conditions for a Standard Run .62

Table 3.2 Typical Liner-Panel and Plenum Operating Conditions for a Standard Run......62

Table 3.3 Summary of Uncertainty Analysis

Table 4.1 The Number of Holes in Each Liner-Panel and Dilution Row, Including their Respective Discharge Coefficients and Diameters

Table 4.2 Sizes and Locations of the Thermal Measurement Planes

Table 4.3 Sizes and Locations of the Flow Field Planes. .91

Table 5.1 Comparison Between the Experimental and Computational Conditions for the Combustor Simulator

Table A.1 Pressure Transducer Specifications; Omega Serial Number: 60518167 169

Table A.2 Pressure Transducer Specifications; Omega Serial Number: 60518721 ...........170

Table A.3 Pressure Transducer Specifications; Setra Serial Number: $1083663 \ldots \ldots \ldots \ldots \ldots . . . .171$

Table A.4 Pressure Transducer Specifications; Setra Serial Number: $1083664 \ldots \ldots \ldots \ldots \ldots . . .172$

Table A.5 Pressure Transducer Specifications; Setra Serial Number: $1185966 \ldots \ldots \ldots \ldots \ldots . . . .173$

Table A.6 Pressure Transducer Specifications; Setra Serial Number: $1185967 \ldots \ldots \ldots \ldots . . . . . .174$

Table A.7 Pressure Transducer Specifications; Setra Serial Number: $1258460 \ldots \ldots \ldots \ldots \ldots . . .175$

Table A.8 Pressure Transducer Specifications; Setra Serial Number: $1548165 \ldots \ldots \ldots \ldots \ldots . . .176$

Table A.9 Pressure Transducer Specifications; Setra Serial Number: $1753678 \ldots \ldots \ldots \ldots \ldots \ldots . . .177$

Table A.10 Pressure Transducer Specifications; Setra Serial Number: $1753679 \ldots \ldots \ldots \ldots . . .178$ 


\section{List of Figures}

Figure 1.1 Schematic of Hero's engine, which is probably the earliest form of jet reaction (Rolls-Royce, 1992) ................................................................

Figure 1.2 A graphic of a Whittle-type turbo-jet engine (Rolls-Royce, 1992) .........4

Figure 1.3 Pratt \& Whitney's F-119 turbofan engine (courtesy of Pratt \& Whitney)

Figure 1.4 Pratt and Whitney's JT9D PW4000 combustor containing inlet swirlers, dilution holes, and film-cooling holes and slots (Pratt \& Whitney, 1988) .5

Figure 2.1 Diagram of a typical annular combustor (Pratt \& Whitney, 1988) and the damage that can occur to a first stage turbine vane.....

Figure 2.2 Schematic of the combustor simulator used by Koutmos and McGuirk (1989)

Figure 2.3 Schematic of combustor simulator used by Ames and Moffat (1990) ....23

Figure 2.4 Schematic of Stevens and Carotte (1990) test facility .24

Figure 2.5 Graph of the temperature measurements by Stevens and Carotte (1990) showing the kidney-shaped profiles

Figure 2.6 Test section used by Liou and Wu (1992) .25

Figure 2.7 Schematic showing Anacleto, Heitor, and Moreira (1996) combustor model.

Figure 2.8 Schematic of Doerr, Blomeyer, and Hennecke (1997) combustor model.

Figure 2.9

Ahmed (1998) combustor simulator model

Figure 2.10 Schematic showing Gritsch, Martiny, Schulz, Kim, and Wittig (1998) combustor simulator model.

Figure 2.11 Schematic of simulator used by Van Fossen and Bunker (2001) ...........28

Figure 2.12 Schematic side view of the combustor simulator used by Barringer, Richard, Walter, Stitzel, and Thole (2002). 
Figure 2.13 Schematic view of the combustor rig used by Van Fossen and Bunker (2002). On the right is the heat transfer model and on the left is a cross-sectional view of the rig

Figure 2.14 Schematic of Busnaina (1987) combustor simulator modeled by CFD .30

Figure 2.15 Schematic of Holdeman (1993) combustor simulator with different hole shapes, orientations, and patterns tested appearing below

Figure 2.16 Contours of the non-uniform temperature profile measured at the exit of a reacting combustor presented by Halls (1970) .................................32

Figure 2.17 Schematic of Bicen, Tse, and Whitelaw (1988) combustor model...........32

Figure 2.18 Contours of the temperature results obtained by Bicen, Tse, and Whitelaw (1988)

Figure 2.19 Schematic of the reacting combustor model used by Richardson and Samuelson (1992)

Figure 2.20 Schematic of Goebel, Abauf, Lovett, and Lee (1993) combustor simulator

Figure 2.21 Crocker, Smith, and Myers (1994) reacting Allied Signal F109 combustor. .35

Figure 2.22 Schematic of the can combustor modeled by Ebbinghaus and Swithenbank (1995)

Figure 2.23 Schematic of PW4098 combustor model created for validation of the newly created CFD system by Malecki et al. (2001).................................36

Figure 2.24 A picture of the PW6000 engine and its computational combustor model. This study used the CFD system developed by Malecki et al. (2001) to design the PW6000. Following its design, a full-scale operating test rig was constructed and the computational results were validated against experimental measurements. All design and analysis on the PW6000 was done by Snyder et al. (2001).

Figure 2.25 Combustor models used by Goldstein, Lau, and Leung (1983), which include their first combustor with the entry holes for the secondary air arranged axisymmetrically, and the General Electric Company combustor with secondary holes arranged non-symmetrically. 
Figure 2.26 Schematic of the combustor model used by Cameron, Brouwer, Wood, and Samuelson (1989)........................................................................38

Figure 2.27 Schematic of the test facility (above) used by Sinha, Bogard, and Crawford (1990) with an up-close graphic of the film-cooling test section

Figure 2.28 Schematic of the jet in crossflow model developed by Sgarzi and Leboeuf (1997).

Figure 2.29 Schematic of Rydholm (1998) test rig. .41

Figure 3.1 Overall schematic of the large-scale, low-speed, closed-loop wind tunnel facility

Figure 3.2 Perforated aluminum plate with 7.2\% open area (dimensions are in centimeters).

Figure 3.3a, b Schematics of the combustor simulator and turbine vane cascade showing (a) top view, and (b) side view (dimensions are in centimeters).

Figure 3.4 Schematics of an arbitrary liner panel showing the film-cooling hole pattern (Barringer, 2001)

Figure 3.5 Cut-away of combustor simulator showing the plenum supply chambers, and the locations of the flow control mechanisms. Also included are pictures of the flow control mechanisms for panels 3 and 4 , as well as the second row of dilution.

Figure 3.6a, b Wiring connections for a single heater bank in the (a) Wye and (b) Delta configuration $\left(R_{1}, R_{2}\right.$, and $R_{3}$ are the effective resistance of two strip heater elements in parallel)

Figure 3.7 Detailed picture of the pressure transducer box

Figure 3.8 Isometric, side and top pictures of the 24-channel, three-wafer scanivalve.

Figure 3.9a, b (a) Detailed view of the 21-probe, type E thermocouple rake and (b) a photo of the 17-probe, type E thermocouple rake

Figure 3.10a-d Data acquisition system including the (a) SCXI-1303 terminal block, (b) SCXI-1100 module, (c) SCXI-1000 chassis, and (d) NI AT-MIO16E-2 DAQ card (National Instruments, 2002). 
Figure 3.11 Overall laser Doppler velocimeter for a two-component system (TSI, 1995)

Figure 3.12 Atomized olive oil seed generator used in LDV measurements

(Radomsky, 2000)

Figure 3.13 Two-component LDV system set-up and orientation in regards to the combustor simulator.

Figure 3.14 Two-component velocity transformation diagram.

Figure 3.15 Three-component LDV system set-up and orientation in regards to the combustor simulator.

Figure 3.16 Three-component non-orthogonal to orthogonal transformation

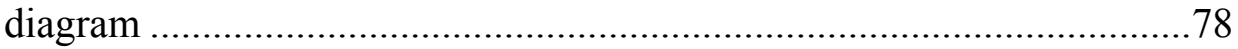

Figure 3.17 Three-component tilt transformation diagram ......................................79

Figure 4.1a, $\mathrm{b} \quad$ A graphical representation of the mass-averaged planes with graphs showing the variations in (a) velocity and (b) mass flow rates along the combustor simulator for a typical run.....

Figure 4.2 Global coordinate system within the combustor

Figure 4.3 Location of the measured thermal field planes . .94

Figure 4.4 Location of the measured flow field planes

Figure 5.1 Temperature profile showing symmetry at the trailing edge of plane $1 \mathrm{~s}$ $(X / L=0.50)$

Figure 5.2 Temperature contours showing symmetry in the pitchwise direction for plane $1 \mathrm{p}$

Figure 5.3 Velocity measurements demonstrating symmetry and periodicity between dilution jets in the pitchwise direction. Profile was taken for plane $1 \mathrm{~s}$

Figure 5.4 Proof of repeatability and velocity transformations for planes $1 p$ and $2 \mathrm{~s}$

Figure 5.5 Film-cooling thermal field measurements at $X / L=0.24$ in plane $0 \mathrm{p}$ for $I=3, M=1.8$ and $D R=1.08$ 
Figure 5.6

Figure 5.7a

Figure 5.7b

Figure 5.8

Figure 5.9

Figure 5.10

Figure 5.11a, b

Figure 5.11c

Figure 5.12

Figure 5.13

Figure 5.14

Figure 5.15
Thermal field measurements in plane $0 \mathrm{p}$ for $I=9, M=3.2$ and $D R=$ 1.12 .......

Cross-pitch temperature contours in plane $1 \mathrm{p}$, downstream of dilution row one for (a) $I=3, M=1.8$ and $D R=1.08$, and (b) $I=9, M=3.2$ and $D R=1.12$. The dilution jet injection remained the same at $I=128$ and $M=12$, while the $D R$ was equal to those of the film-cooling jets

Cross-pitch temperature contours in plane $1 \mathrm{p}$, downstream of dilution row one for (a) $I=3, M=1.8$ and $D R=1.08$, and (b) $I=9, M=3.2$ and $D R=1.12$. The dilution jet injection remained the same at $I=128$ and $M=12$, while the $D R$ was equal to those of the film-cooling jets

Streamwise temperature contours through a high-momentum dilution jet in plane $1 \mathrm{~s}$. $I=128, M=12$, and $D R=1.12$ for the dilution jet

Three-component velocity measurements for plane $1 \mathrm{p}$ showing contours of $u / u_{\text {in }}$ with secondary velocity vectors $v / u_{\text {in }}$ and $w / u_{\text {in }} . I=$ $128, M=12$, and $D R=1.12$ for the dilution jets

Secondary flow vectors of $v / u_{\text {in }}$ and $w / u_{\text {in }}$ with temperature contours within plane $1 \mathrm{p}$

Contours of the rms fluctuations in plane $1 \mathrm{p}$; (a) $u_{r m s} / u_{\text {in }}$, (b) $v_{r m s} / u_{\text {in }}$ and (c) $w_{r m s} / u_{i n} . I=128, M=12$, and $D R=1.12$ for the dilution jets

Contours of the rms fluctuations in plane $1 \mathrm{p}$; (a) $u_{r m s} / u_{i n}$, (b) $v_{r m s} / u_{\text {in }}$ and (c) $w_{r m s} / u_{i n} . I=128, M=12$, and $D R=1.12$ for the dilution jets

Contours of the normalized combustor turbulent kinetic energy, $k / u_{i n}^{2}$,

in plane $1 \mathrm{p}$.

Contours of the turbulence levels, $T L$, in plane $1 \mathrm{p}$

Contours showing the local turbulence levels, $T u$, within plane $1 \mathrm{p}$ (normalized mass-averaged velocity $u / u_{\text {in }}=1.55$ ).

Streamwise normalized temperature contours for plane $2 \mathrm{~s}$, beginning slightly before the second dilution row and extending through the combustor exit. The dilution jet has an $I=32, M=6$, and $D R=1.12 \ldots .132$ 
Figure 5.16

Temperature contours for plane $2 \mathrm{p}$ at one dilution hole diameter downstream $\left(1 D_{2}\right)$. The first row of dilution has an $I=128$ and $M=$ 12 , while the second row has an $I=32$ and $M=6$. The $D R=1.12$ for the film-cooling and both rows of dilution

Figure 5.17 Streamwise, $u / u_{i n}$, and vertical, $w / u_{i n}$, vectors for plane 2 s. $I=32, M=$ 6 , and $D R=1.12$ for the dilution jet

Figure 5.18 Vectors of $u / u_{\text {in }}$ and $w / u_{\text {in }}$ overlaying temperature contours within plane 2 s. $I=32, M=6$, and $D R=1.12$ for the dilution jet.

Figure 5.19a Contours of the rms fluctuations for the flow vectors in plane $2 \mathrm{~s}$; (a) $u_{r m s} / u_{i n}$ and (b) $w_{r m s} / u_{i n}$. The dilution jet has an $I=32, M=6$, and $D R$ $=1.12$

Figure 5.19b Contours of the rms fluctuations for the flow vectors in plane $2 \mathrm{~s}$; (a) $u_{r m s} / u_{i n}$ and (b) $w_{r m s} / u_{i n}$. The dilution jet has an $I=32, M=6$, and $D R$ $=1.12$

Figure 5.20 Contours of the normalized combustor turbulent kinetic energy, $k / u_{i n}^{2}$, in plane $2 \mathrm{~s}$

Figure 5.21 Turbulence levels, $T L$, within plane $2 \mathrm{~s}$

Figure 5.22 Contours of the local turbulence level, $T u$, within plane $2 \mathrm{~s}$

Figure 5.23 Three-component velocity measurements for plane $2 p$, post-dilution row two; contours of $u / u_{\text {in }}$ and vectors of $v / u_{\text {in }}$ and $w / u_{\text {in }}$. The second row dilution jet has an $I=32, M=6$, and $D R=1.12$, while the first row dilution jet has an $I=128, M=12$, and $D R=1.12$

Figure 5.24 Vectors of $v / u_{i n}$ and $w / u_{\text {in }}$ overlying the temperature contours within plane $2 \mathrm{p}$ (post-dilution row two). $I=32, M=6$, and $D R=1.12$ for the second row dilution jet, and $I=128, M=12$, and $D R=1.12$ for the first row dilution jet

Figure 5.25a-c Contours of the rms fluctuations for the flow field in plane 2p; (a) $u_{r m s} / u_{i n}$, (b) $v_{r m s} / u_{i n}$, and (c) $w_{r m s} / u_{i n}$ (second row $I=32, M=6$, and $D R$ $=1.12)$.

Figure 5.26 Contours of the combustor turbulent kinetic energy, $k / u_{i n}^{2}$, in plane $2 \mathrm{p}$. 144

Figure 5.27 Contours of the turbulence levels, $T L$, in plane $2 p$ 145 
Figure 5.28 Contours of the local turbulence levels, $T u$, within plane $2 p$

(normalized local mass-averaged velocity $u / u_{\text {in }}=2.60$ )

Figure 5.29 A comparison of normalized temperature contours measured

experimentally to those predicted computationally in plane $1 \mathrm{p}$

Figure 5.30 Comparison of normalized velocity plots showing contours of $u / u_{i n}$, and vectors of $v / u_{i n}$ and $w / u_{\text {in }}$ for plane $1 \mathrm{p}$

Figure 5.31a Comparison of temperature contours for plane 2s; (a) experimental, (b)

computational.

Figure 5.31b Comparison of temperature contours for plane 2s; (a) experimental, (b) computational.

Figure 5.32a Comparison of $u / u_{\text {in }}$ and $w / u_{\text {in }}$ velocity vectors plane $2 \mathrm{~s}$; (a)

experimental, (b) computational

Figure 5.32b Comparison of $u / u_{i n}$ and $w / u_{\text {in }}$ velocity vectors plane $2 \mathrm{~s}$; (a)

experimental, (b) computational

Figure 5.33 Computational predictions of the streamlines from dilution row one (Stitzel, 2001).

Figure 5.34 Comparison of normalized temperature contours for plane $2 p$

Figure 5.35 Comparison of normalized velocity plots showing contours of $u / u_{i n}$, and vectors of $v / u_{\text {in }}$ and $w / u_{\text {in }}$ for plane $2 \mathrm{p}$.

Figure B.1 TSI's recommended three-component non-orthogonal to orthogonal velocity transformation. This diagram shows their coordinate system, probe orientation, and variables (TSI, 2000)

Figure B.2 TSI's recommended three-component tilt transformation, including their specific coordinate system, probe orientation, and variables (TSI, 2000)

Figure B.3 The two-component transformation used in this study, including the specific coordinate system, probe orientation, and variables defined for the combustor simulator described in Chapters 3 and 4 (TSI, 2000)

Figure B.4 Three-component non-orthogonal to orthogonal transformation used in this study. Again, showing the coordinate system, probe orientation, and variables specific to this study (TSI, 2000). 
Figure B.5 The three-component tilt transformation used in this study, including the coordinate system, probe orientation, and variables specific to this study (TSI, 2000) ................................................................... 184 


\section{Chapter 1: Introduction}

The history of turbine propulsion dates back as early as 150 B.C. when an Egyptian philosopher and mathematician named Hero possibly invented the first steam powered engine, the aeolipile (Figure 1.1). Used as a toy, the aeolipile was a simple closed spherical vessel that was mounted on bearings, which allowed it to spin in circles. The spinning occurred from the tangential force imparted by the steam discharging from the vessel's nozzles (Wilson et al., 1998). Later in the $13^{\text {th }}$ century, historians documented the use of rockets by the Chinese to ward of their enemies. It was not until centuries later, however, in 1930 that Frank Whittle was granted his first patent for using a gas turbine, shown in Figure 1.2, to produce a propulsive jet (Rolls-Royce, 1992). Propulsion is a practical application of Sir Isaac Newton's $3^{\text {rd }}$ Law of Motion, which states that for every force acting upon a body there is an equal and opposite force. In aircraft propulsion, the body is the air that is accelerated through an engine. The force required for this acceleration imparts an equal force in the opposite direction on the airplane, thus making flight as we know it today possible.

Introduced in 1939, the first gas turbine aero-engine was capable of producing 4.9 $\mathrm{kN}$ of thrust and propelling its aircraft to maximum speeds of $700 \mathrm{~km} / \mathrm{hr}$. Today, modern gas turbine aero-engines, shown in Figure 1.3, can produce $156 \mathrm{kN}$ of thrust and enable aircrafts to reach speeds in the excess of $3200 \mathrm{~km} / \mathrm{hr}$. The highly competitive gas turbine industry has recently been driven by demands for higher power-to-weight ratios, increased thermal efficiencies, and reliability while maintaining affordability. In its continual quest, the industry must constantly try to raise the turbine inlet temperature, which according to the well-known Brayton cycle is key to higher engine efficiencies. The desire and need for increased turbine inlet temperatures creates an extremely harsh environment for the combustor liner-panels in addition to the components downstream of the combustor.

The flow within the combustor itself is no simple feat to model and understand.

Figure 1.4 shows a PW4000 engine combustor that contains inlet swirlers, dilution holes, and film-cooling holes and slots. Shear layers between the dilution jets and the mainstream, as well as combustor liner film-cooling interactions create a highly complex, 
but not random, mean flow field within the combustor. Clearly, the myriad of choices regarding film-cooling and dilution row schemes do not alleviate the situation. A completely uniform temperature and velocity profile at the combustor exit is naturally desirable from the standpoint of reducing the secondary flows; however, this is often not the case due to a lack of thorough mixing within the combustor. Poor mixing can result in non-uniformities, such as hot streaks, and allow non-combusted fuel to exit the combustor. Hot streaks can lead to premature wear and failure or turbine components, while non-combusted fuel entering into the turbine can mix with film-cooling flow leading to catastrophic failures of the engine.

Until recently, compressor, combustor, and turbine component studies were conducted independently of one another. Moreover, the flow within the first vane of the turbine is rather complex. The secondary flows that develop in this region often have adverse effects on heat transfer and lead to premature failure of the endwall and turbine vanes. These secondary flows typically develop as a result of the exiting combustor temperature and velocity profiles. Therefore, to accurately model these secondary flows within the turbine, the proper combustor exit temperature and velocity profiles are critical.

The purpose of this investigation was to develop a database documenting the thermal and flow characteristics within a combustor simulator for a typical gas turbine aero-engine. Specifically, the flow, thermal, and mixing characteristics of film-cooling and dilution flows within the combustor are of interest. This database is also valuable in benchmarking computational predictions. With a greater understanding of these phenomena, designers will better able to predict the behavior and mixing characteristics of combustors. This understanding will also aid in reducing non-uniformities before entering the turbine vane cascade. In the long run, an optimized combustor design will help to maximize the effectiveness of the coolant along the turbine vane endwall and finally provide a coherent understanding of the interaction between the two engine components.

The combustor simulator used in my study is unique in that it is a linear representation of a true Pratt and Whitney aero-engine. The simulator combines the interaction of two rows of dilution jets, which are staggered in the streamwise direction 
and aligned in the spanwise direction, with that of film-cooling along the combustor liner walls. Furthermore, the flow conditions applied in this study are representative of an actual aero-engine. The current literature does not include any data on the thermal and flow field distributions for high momentum flux dilution jets combined with low momentum flux film-cooling jets. In particular, three-component velocity measurements and corresponding temperature planes are presented in this thesis. Detailed turbulence measurements are also presented in an ongoing effort to better understand the mixing characteristics of the aero-engine combustor.

A broad literature search (Chapter 2) was conducted to organize and compile the large amounts of data already in existence for non-reacting and reacting combustor simulators, as well as the characteristics of jets injected into a crossflow. Chapter 3 presents an overview of the wind tunnel facility used for my study, including details on the combustor simulator, the thermal conditioning system, and typical operating conditions. The various measurement instruments and techniques used in this research are also described, while the last section of Chapter 3 presents an uncertainty analysis. A description of the experimental test matrix is given in Chapter 4, including flow calculations, measurement planes, the global coordinate system, and normalization techniques. Chapter 5 presents the thermal and flow field results and concludes with comparisons of experimental data to computational results obtained by Stitzel (2001). The conclusions and recommendations are finally discussed in Chapter 6. 


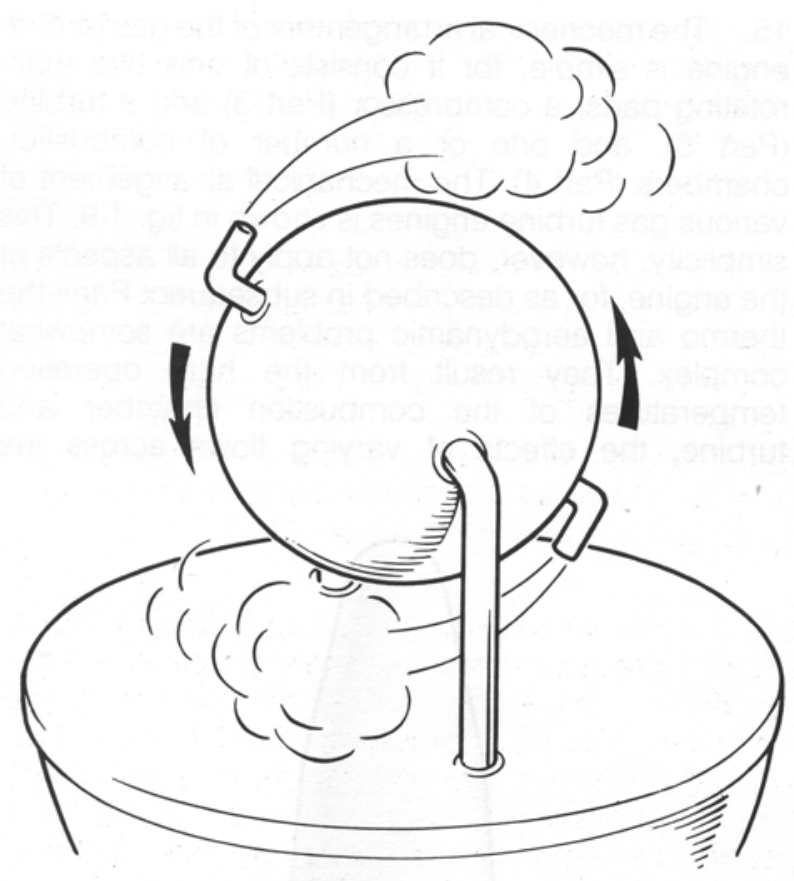

Figure 1.1 Schematic of Hero's engine, which is probably the earliest form of jet reaction (Rolls-Royce, 1992).

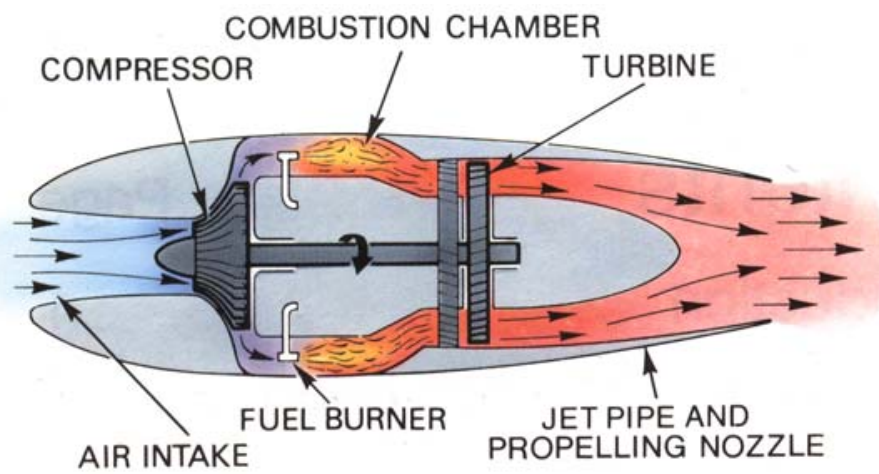

Figure 1.2 A graphic of a Whittle-type turbo-jet engine (Rolls-Royce, 1992). 


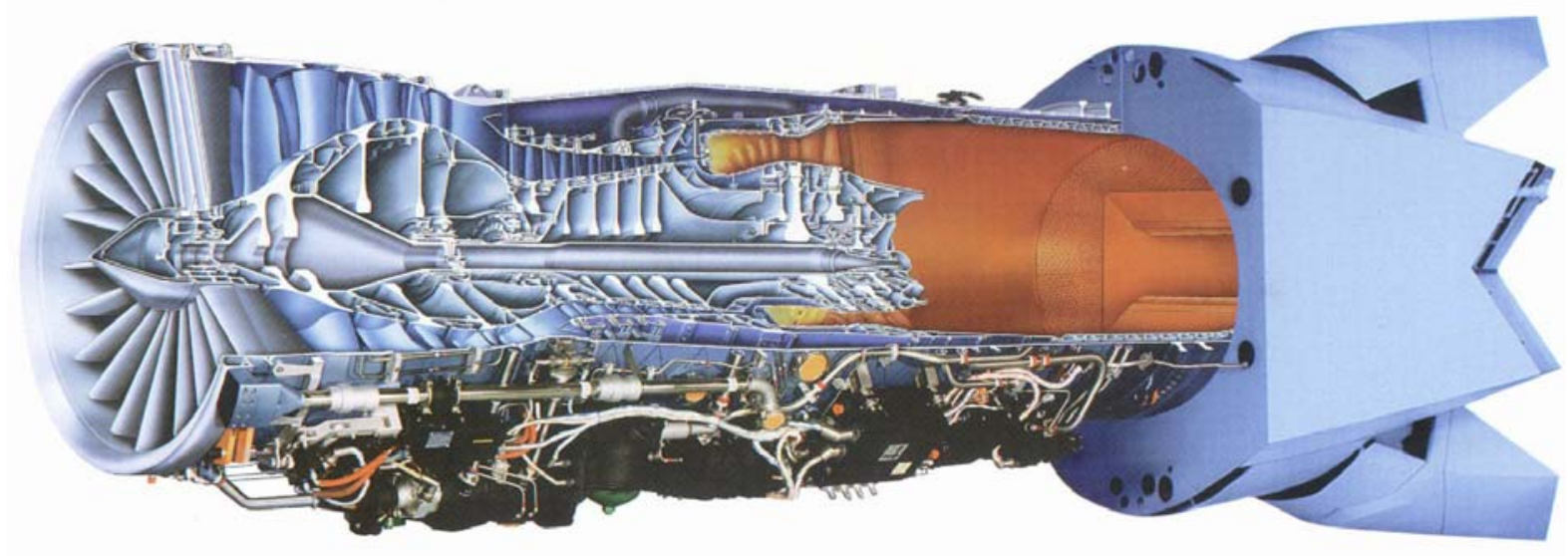

Figure 1.3 Pratt \& Whitney's F-119 turbofan engine (courtesy of Pratt \& Whitney).

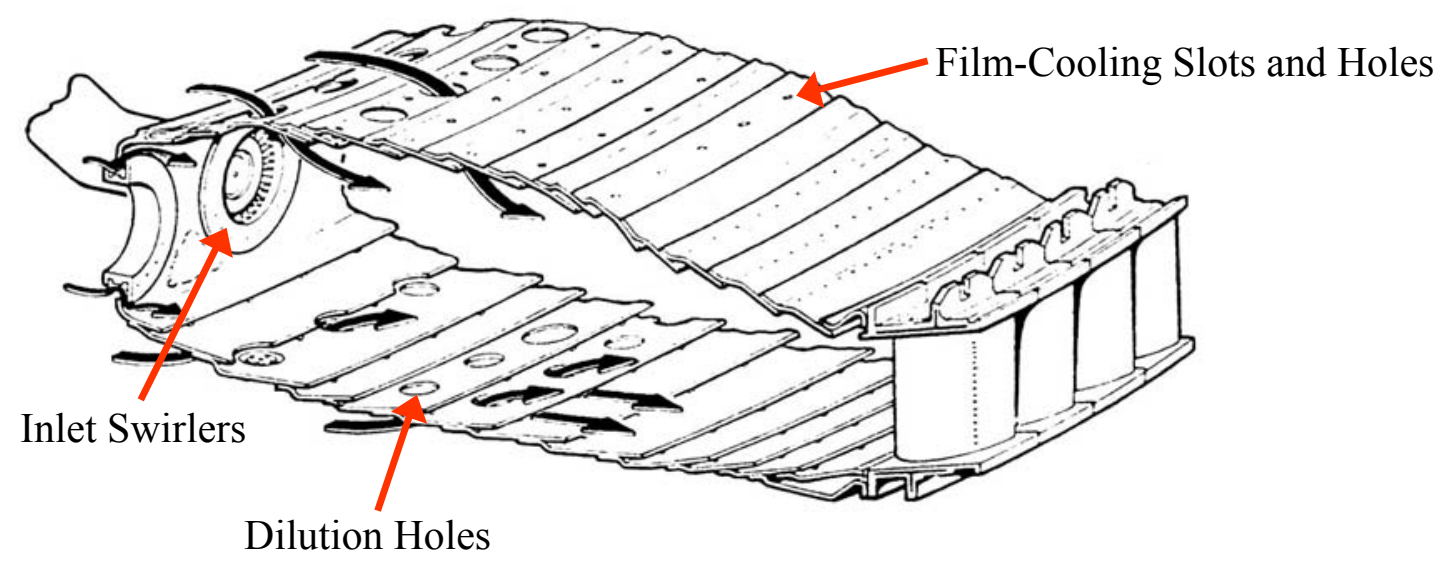

Figure 1.4 Pratt and Whitney's JT9D PW4000 combustor containing inlet swirlers, dilution holes, and film-cooling holes and slots (Pratt \& Whitney, 1988). 


\section{Chapter 2: Review of Relevant Literature}

The tasks of a combustor are by far the most complex and demanding within a gas turbine engine. Fuel, supplied by fuel spray nozzles, along with great quantities of air, provided by the compressor, must be thoroughly mixed, completely burned, and made uniform before entering the turbine. These tasks must be accomplished with a minimum loss of pressure and the maximum release of heat within the limited available space.

A number of studies have shown that the flow field exiting a combustor is highly non-uniform in pressure, velocity and, most importantly, temperature. Hot streaks amongst other non-uniformities push the thermal limits of the first stage of turbine turning vanes immediately downstream of the combustor exit. In particular, these nonuniformities can have detrimental effects on engine performance and cause a reduction in the expected life of critical components such as the turbine vanes; a pictorial representation is shown in Figure 2.1. Furthermore, studies have also shown that swirlers and the flow rates through film-cooling and dilution holes can immensely alter and influence the combustor exit profiles. A broad literature search was conducted to compile the large amounts of data in existence and to highlight the many combustor characteristics mentioned above. The first section of this chapter presents experimental and computational studies conducted in non-reacting combustor simulators. The second and third sections present studies on reacting combustors, and the fourth section presents studies on the characteristics of jets injected into a crossflow. It will quickly become apparent from the information shown in this section that the combustion characteristics are not as dominant as the influence of swirlers, film-cooling flow and dilution flow on the various profiles at the exit of the combustor. This dominance is important, for it lends credibility to our non-reacting simulations.

\subsection{Experimental Studies and Computational Simulations for Non-Reacting Flows in Combustor Simulators}

Koutmos and McGuirk (1989) studied a non-reacting water channel, shown in Figure 2.2, consisting of a swirl driven primary zone, annularly fed dilution jets, and an 
exit contraction nozzle. It was found that a reduction in the swirler exit area resulted in a decrease in the strength of the recirculation and turbulence in the primary zone due to the weak penetration by the first row of dilution jets. Also noted was the rapid and efficient mixing of the first and second rows of dilution, which led to a reduction in velocity and turbulence non-uniformities at the combustor exit. Turbulence levels between 85 and $87 \%$ were measured within the dilution region, while a reduction of $45 \%$ in the turbulence level was noted between the dilution region and the combustor exit.

Using a non-reacting, simulated combustor flow field (see Figure 2.3) that contained wall slots, two rows of in-line dilution, and a 2:1 contraction, Ames and Moffat (1990) found turbulence levels as large as 19\%. In addition integral length scales of 4 to 6 centimeters were determined. These length scales were found to be on the order of the dilution hole diameters. Lastly, both the turbulence levels and the length scales were found to have a significant impact on the heat transfer in the leading edge region of the first vane.

Stevens and Carotte (1990) conducted an experimental investigation on the development of the combustor dilution zone and jets by focusing on the downstream thermal field within a non-reacting, annular combustor simulator shown in Figure 2.4. The model contained a row of sixteen heated jets that were injected normally into a confined annular crossflow. The jets were heated to identify the trajectory and spreading of the core. Furthermore, the air was fed to the holes along a representative approach annulus. Despite the uniformity of the approach flow, the velocity profile across the exit plane of each dilution hole varied in a random manner. Temperatures distributions measured at a plane two hole diameters downstream showed the characteristic kidneyshaped profile, yet an apparent lack of symmetry existed (Figure 2.5). Velocity distributions at the same location indicated a corresponding asymmetry in the double vortex structure formed in the wake of the jet. Reasons for these variations were found to most likely be due to slight inconsistencies physically between holes, which in turn affect the exit velocity profiles and ultimately the temperature. Since each jet had its own mixing characteristics, an irregular temperature pattern around the dilution annulus was noted. 
Liou and $\mathrm{Wu}$ (1992) constructed a non-reacting combustor simulator with an isothermal flow field (Figure 2.6). The major finding from this study was that turbulence was inhomogeneous and anisotropic throughout most of the combustor.

By conducting detailed velocity measurements, Anacleto, Heitor, and Moreira (1996) studied the flow patterns in typical rich-burn, quick-quench, lean-burn (RQL) gasturbine combustor simulated with a non-reacting water model of a can-type combustor (see Figure 2.7). The study consisted of a basic flow configuration with changes in the swirl level within the rich zone, geometry of the quench holes, and the momentum of inflowing jets. A primary finding was that the impingement of opposed jets greatly enhanced turbulence production leading to large velocity fluctuations.

Doerr, Blomeyer, and Hennecke (1997) also simulated the rich-burn, quick quench, lean-burn combustion concept with the combustor model shown in Figure 2.8. This was accomplished experimentally by investigating a non-reacting multiple jet configuration with a confined crossflow. One row of opposing in-line jets was injected perpendicularly into a heated crossflow within a rectangular test-section. The spacing and size of the holes were varied and modeled after realistic combustors. The analogy between heat and mass-transfer allowed for the time-averaged temperature distributions to be deduced, which in turn served as an indicator of mixing. After determining the temperature distributions and mixing rates at several downstream locations, the best mixing in terms of the temperature uniformity was found to be highly dependent on the momentum flux of the jets. Later in 1999, using the same simulator with slight modifications, Blomeyer, Krautkremer, Hennecke, and Doerr tested the effects of swirl in addition to the high momentum flux jets. The addition of swirl to mainstream produced high turbulence in the shear layer of the jets. Furthermore, it was found that jet penetration increased with increasing ratios of duct height to jet diameter and with increased relative spacing. Again, the mixing was found to be the best for in-line jets opposing one another.

Using a two-component LDV system, Ahmed (1998) studied the effects and properties of a confined, isothermal, swirling flow field in an axisymmetric, non-reacting research combustor (Figure 2.9). Only the effects of swirling the flow at the inlet of the combustor were considered. In all, the major findings indicate that swirling enhances the 
production and distribution of turbulent energy in the combustor, which directly correlate to the degree of thorough flow mixing. At $0.75 D(D=$ swirler outer diameter $)$ downstream of the swirler, turbulence levels of $87 \%$ were measured at the combustor centerline with levels reaching as high as $92 \%$ slightly above the centerline. At $18 D$ downstream of the swirler, the turbulence levels had decayed to a maximum of $52 \%$ at the combustor centerline. However, one must keep in mind that this study did not address the effects of dilution jets coupled with swirling. Dilution jets are often used to counteract the effects of swirling at the inlet.

Gritsch, Martiny, Schulz, Kim, and Wittig (1998) determined the effects of dilution jets on slot film-cooling in a non-reacting simulated combustor liner shown in Figure 2.10. A scaled up test section was constructed consisting of a flat wall with a film-cooling slot and a perpendicular dilution jet downstream. A representative Reynolds number (5800) was used and three cases were studied. Slot-cooling momentum fluxes, $M$, and dilution momentum fluxes, $I$, were varied as follows: $M=1$ and $I=10, M=1$ and $I=7$, and $M=2$ and $I=7$. Using light sheet visualization, jet penetration was found to be the highest for the highest jet momentum flux and the lowest slot-cooling momentum flux. Furthermore, it was determined that the coolant layer lifted off of the wall downstream of the dilution jet leading to less protection in this area. The reasoning for this phenomenon was that the counter-rotating vortices induced by the jet interaction with the main flow were swooping down and lifting the coolant away from this region. Within my study an alternate reason is proposed based on two and three component LDV measurements. Finally, high heat transfer coefficients, reported downstream of the dilution jet injection, were found to gradually decrease along the remaining length of the combustor.

Using an approximately half-scale model, shown in Figure 2.11, of a can-type combustor for a low $\mathrm{NO}_{\mathrm{x}}$, ground based power-generating turbine, Van Fossen and Bunker (2001) conducted heat transfer measurements on a flat plate with a circular leading edge. The circular leading edge was intended to simulate the lead edge of a first stage turbine vane. The effects of the combustor at a plane perpendicular to the mainstream through the blades stagnation region were quantified. The non-reacting tests were conducted with room temperature air that flowed through six vane swirlers. 
Furthermore, the combustor simulator contained no dilution holes. Flow field surveys at the model stagnation point showed a single swirling vortex, produced from the six swirlers, with flow angles of up to 36 degrees at the outer edges of the test section. As a result, very high levels of freestream turbulence were generated. The spanwise averaged turbulence intensity was found to be $28.5 \%$. In addition, the turbulence generated was isotropic with the magnitudes of the fluctuating quantities in the axial and cross-stream directions being nearly identical. Average length scales were $3.3 \mathrm{~cm}$ in the axial direction and $2.6 \mathrm{~cm}$ in the cross-stream direction. Lastly, as a result of the turbulent exhaust form the DLN can combustor simulator, an increase by a factor of 1.77 in the stagnation heat transfer was noted.

Barringer, Richard, Walter, Stitzel, and Thole (2002) presented experimental results of the same combustor simulator used in my study; however, these baseline results were done without a downstream turbine vane (Figure 2.12). The focus of the results was on the effects of dilution jets with regards to the downstream velocity and thermal flow fields. The results of these experiments question the assumption of a uniform pressure and endwall turbulent boundary layer at the exit of the combustor. Namely, the total pressure profiles proved the two previously stated assumptions to be invalid. While the dilution jets did reduce the variations in the total pressure and velocity fields, there was still appreciable variation within the near-wall region. Likewise, the thermal field contours suggested that the dilution jets increased the mixing of the coolant, provided by the upstream film-cooling panels, into the mainstream flow. The mixing implied a lack of available cooling along the downstream turbine vane endwall and verified the notion of dilution jets strongly affecting the various fields exiting the combustor. Lastly, the turbulence levels entering the turbine section were between $15-18 \%$, and the turbulent length scales were on the order of the turbulence producing mechanism, i.e. the dilution hole diameter. Both of these assumptions remain consistent with observations in numerous reacting and non-reacting combustor simulator models.

Similar to their study in 2000, Van Fossen and Bunker (2002) again conducted heat transfer measurements in the stagnation region of a flat plate with an elliptical leading edge (see Figure 2.13). In this later study, the elliptical leading edge was intended to simulate the first stage of turbine vanes in a large commercial high-bypass 
turbofan engine. Unlike the can combustor, this combustor model contained two annular rows of fuel-air swirlers that were aligned in the radial direction. Heat transfer and hot wire measurements were made at two circumferential positions; one directly downstream a pair of swirlers and another directly half way between the two pairs of swirlers. The turbulence intensity reported in this study was not the traditional average kinetic energy, rather it was the "absolute intensity" defined as

$$
T u_{a i}=\frac{\sqrt{\overline{\rho u_{r m s}^{2}}+\overline{\rho v_{r m s}^{2}}}}{\rho u}
$$

where $u_{r m s}$ and $v_{r m s}$ are the fluctuating components of velocity in the axial and circumferential directions, respectively; and $u$ is the mean velocity in the axial direction. Because of this definition, the intensities measured in this study are larger than the commonly reported average kinetic energy by a factor of $\sim 1.41$. Nevertheless, the average overall turbulence level was found to be $32 \%$. The ratio of axial to circumferential fluctuations was 1.15, and the axial length scale was $1.29 \mathrm{~cm}$. Lastly, the heat transfer augmentation was found to be between 34 and 59\% over laminar levels.

The following studies concentrate on the results of computational simulations and experimental comparisons for non-reacting flows within combustors. The findings bolster the notion that although computations are beneficial in providing general flow characteristics, the lack of a true turbulence model leaves the need for experiments to truly characterize the mixing and turbulence levels within a combustor.

Busnaina (1987) determined computational results of jets injected into an isothermal combustor flow field using the $k-\varepsilon$ model (Figure 2.14). In particular, jet-tocrossflow velocity ratios were varied and different swirl strengths were investigated. Predicted computational results showed expected trends in the flows such as the jet penetration, deflection, and swirl crossflow effect. However, comparison with flow visualizations showed that while the quality of the predicted results was good, a better turbulence model was necessary. In particular, a turbulence model that better handles complicated swirling and its associated recirculation was needed. 
Holdeman (1993) presented the experimental and computational results of the mixing of single, double, and opposed rows of jets with an isothermal and variable temperature mainstream in a confined subsonic crossflow (see Figure 2.15). The principal goal was to summarize the complex 3-D flow field in the dilution zone of a combustor. The primary finding of the study was that the momentum flux ratio was the single most important parameter in dictating the temperature profiles. Furthermore, the exit temperature distributions for single-sided, single and double rows of jet injection were very similar indicating only slightly better mixing for the double row case (staggered, dissimilar, and in-line). For the cases involving inline, opposed rows of jets, the two streams mixed very rapidly and the effective mixing height was found to be half the duct height for equal momentum fluxes on both sides. For opposed rows of staggered jets, the optimum ratio of orifice spacing to duct height was twice the value for singlesided injection at the same momentum flux. As expected, computational results for opposed, impacting inline jets did not predict mixing very well. The penetration, however, of the jet was reasonably predicted.

Stitzel (2001) presented a thesis in which a computational model of the nonreacting linear combustor used in this study was constructed and simulated (Figure 2.12). The flow simulations were accomplished using the RNG $k-\varepsilon$ model in the Fluent computational package. Comparisons between these computational results and the experimental results in this study are made in Chapter 5. These comparisons will address the accuracy in the prediction of characteristics such as the penetration heights and jet mixing characterized by the non-uniformity or uniformity of the temperature and velocity profiles throughout the combustor simulator.

\subsection{Reacting Combustor Experimental Studies and Computational Simulations}

In 1970, Halls conducted one of the first reacting experiments for a Conway engine. The results, consistent with non-reacting outcomes, indicated a highly nonuniform temperature profile at the combustor exit and are shown in Figure 2.16. 
Bicen, Tse, and Whitelaw (1988) conducted experiments for two different air-fuel ratios and two different geometric arrangements to determine the combustion characteristics for an annular combustor (see Figure 2.17). The combustor consisted of two rectangular sectors that incorporated slot film-cooling and two rows of dilution jets. One geometry consisted of positioning the vaporizer in line with the primary dilution holes, which were staggered in relation to the secondary dilution holes. The other had the primary dilution holes in line with the secondary dilution holes, and both were out of line with respect to the vaporizer. Both models indicated highly non-uniform distributions of velocity and turbulence levels near $20 \%$ over the majority of the exit plane. Furthermore, localized hot spots, shown in the temperature distributions (Figure 2.18), and velocity peaks were attributed to the opposing rows of dilution jets that prevent the penetration of the mainstream crossflow. Note that these turbulence levels were consistent with those measured in non-reacting simulators; for example, Barringer et al. (2001) measured turbulence levels between $15-18 \%$ at the exit of the combustor simulator used in this study.

In 1992, Richards and Samuelsen presented results of experiments conducted to investigate the primary dilution jets and their effect on the overall performance of the combustor. Using a reacting model combustor, shown in Figure 2.19, containing entrance swirl and two roles of dilution jets, the effects of the location of the first row of dilution hole relative to the dome swirler were tested. The effect of the number of jets in the primary row was also investigated. Thermal and flow fields were measured with the principle finding being that the location of the primary holes significantly affected the percent mass recirculated into the dome region. Consistent with earlier studies, the momentum flux greatly affected the mixing, uniformity and efficiency of the combustor. Furthermore, the increase in the number of jets had a significant effect on the mixing uniformity around the duct's circumference.

Goebel, Abauf, Lovett, and Lee (1993) conducted experimental measurements of velocity, turbulence, and temperature profiles downstream of the reacting small-scale combustor simulator seen in Figure 2.20. The effects of nozzle swirl, liner mixing and dilution holes were studied, and the operating conditions, such as mass flow, pressure, and combustion, were all qualified independently. Appreciable combinations of swirl 
and dilution jets reduced the turbulence levels. Without swirl and at low swirl levels, the dilution jets significantly increased the turbulence levels. Also established was the effect of opening and closing the dilution jets. It was found that this action greatly affected the combustor exit velocity and temperature profiles as noted by Barringer et al. (2001) in a non-reacting study. Lastly, for low swirl velocities, i.e. no swirler, the turbulence levels were also found to be consistent with and without combustion. Along the centerline, the swirl turbulence intensity ranged from 5 to $5.5 \%$ with and without combustion, respectively, while the axial turbulence intensity remained constant at $\sim 5.3 \%$ for both scenarios.

Crocker, Smith, and Myers (1994) investigated ways to reduce the temperature pattern factor at the combustor exit of a reverse flow annular Allied Signal F109 combustor (Figure 2.21). The temperature pattern factor is an indication of uniformity at the combustor exit. Qualitatively, the pattern factor is the difference between the radially averaged temperature at the combustor exit divided by the difference between the radially averaged temperature at the combustor exit and the combustor inlet temperature. The equation for the temperature pattern factor is given by

$$
P F=\frac{T_{\max }-T_{\text {exit }}}{T_{\text {exit }}-T_{\text {inlet }}}
$$

where $T_{\max }$ is the maximum spatial combustor exit temperature, and $T_{\text {exit }}$ and $T_{\text {inlet }}$ are the averaged combustor exit and inlet temperatures, respectively. The baseline case indicated non-uniformities at the combustor exit. These non-uniformities were attributed to fuel flow variations. However, upon modification to the dilution jets, i.e. angling the dilution in the outer liner opposite to those on the inner liner, significant reductions in the non-uniformity at the turbine inlet were noted. This study is consistent with other claims that the dilution flow can be greatly adapted to achieve desired combustor exit profiles.

The following discussion presents studies in which computational simulations and comparisons with experimental results were conducted for reacting combustor flows. In 1993, Lawson computationally modeled a GE high-bypass, turbofan aircraft engine combustor and altered liner film-cooling and dilution flow parameters to achieve target 
combustor exit temperature profiles. In particular, two exit profiles were desired including an inboard peaked profile and a uniform exit temperature profile. The inboard peaked profile was achieved by moving 35 percent of the inner lining cooling in the baseline case to the outer lining. The flat profile was achieved by reducing the liner cooling in the baseline by 20 percent and placing that coolant into the dilution. The determined changes where then applied to actual combustor hardware and full component rig measurements were made. The computational results for the combustor exit temperature profile closely matched experimental results once again proving the effect of dilution and liner cooling on the combustor exit profile, as well as CFD's effectiveness in predicting this behavior. No conclusions, however, were drawn regarding the accuracy of the turbulence levels predicted computationally, as opposed to those measured experimentally. In all, the computational modeling of the system resulted in cost savings in the excess of $\$ 50,000$ and time savings of nearly month.

Through the use of an existing can combustor configuration, Ebbinghaus and Swithenbank (1995) were able to determine the effects of varying the number of primary, secondary, and dilution jets at each axial location throughout the simulator (refer to Figure 2.22). Through comparisons of experimental and computational results, a weakness in the numerical model was noted as an underestimation of mixing behind the dilution jets, which resulted in an over-prediction of the cyclic temperature variations immediately downstream of the jets. However, computational fluid dynamics was noted as being extremely useful in predicting trends in the overall temperature distribution of a can combustor, while the variation in the number of jets was shown to significantly alter the exit temperature profile.

In an effort to develop an aero-engine for the 100-seat aircraft market, Pratt and Whitney used computational fluid dynamics to design and construct a full annular test rig. A Lagrangian fuel spray model along with a combustion model were only two of the major components included in the CFD simulations presented in the first part of this paper by Malecki et al. (2001). Figure 2.23 shows a schematic of the PW4098 combustor model created for validation of the computational system. Upon demonstrating the accuracy of the developed CFD system for three different combustors of the PW4000 engine family, the PW6000 series combustor was designed and constructed. The results 
of the comparison between the predictions and its actual full annular test rig are presented in the second part of this paper by Snyder et al. (2001). Figure 2.24 shows the PW6000 series engine as well as the combustor model used in the computational simulations. In all, several conclusions were drawn from the entire study. The CFD analysis system demonstrated the ability to accurately predict the flow distribution, pressure drop, and exit temperature profile for the PW6000 series engine. Again, similar to many previous studies, a direct correlation between the combustor exit temperature profile and the dilution hole pattern was found. Like Lawson (1993), due to significant cost and time savings, the value and usefulness of computational modeling was once again demonstrated. Lastly, the combustor exit profile was found to be fairly insensitive to the pre-diffuser inlet pressure profiles, which are subject to change over the life of the engine.

\subsection{Non-Reacting and Reacting Studies}

In 1983, Goldstein et al. used a laser Doppler velocimeter to quantify the effects of combustion on two different combustors shown in Figure 2.25. The first combustor was designed specifically as a research tool and used a swirler to introduce the primary air. The entry holes for the secondary air were arranged axisymmetrically and their sizes were designed to obtain a proper pressure distribution. The second combustor was supplied by the General Electric Company, and used holes for the secondary air that were arranged in a non-symmetrical manner. The turbulence levels reported, based upon local velocities, ranged between approximately 30 and 40\%, without combustion, and 12 and $21 \%$, with combustion for the first combustor design. For the second combustor design the turbulence levels ranged from 18 to $29 \%$ without combustion, and 14 to $16 \%$ with combustion. The results illustrated two major findings; the turbulence levels are much greater for axisymmetrically arranged secondary flow holes, and combustion has a sizable effect on the turbulence levels.

Cameron, Brouwer, Wood, and Samuelsen (1989) took measurements of the mean and rms velocities, as well as the mean temperature in a reacting and non-reacting 
model combustor with wall injection (Figure 2.26). In the absence of liquid injection and reaction, flow splits produced a strong on-axis recirculation zone. Amidst the presence of injection and reaction, the on-axis recirculation was absent. RMS levels were significantly increased with an increase in flow velocity, which was dominated by the primary jets. Temperature fields revealed the location of a primary zone upstream the first row of jets, a secondary zone between the jets, and a dilution zone downstream of the two zones where the gasses were cooled. Hot streaks were identified due to nozzle asymmetry. Increases in air to fuel ratios resulted in more symmetric temperature profiles throughout the combustor as well as at the combustor exit.

For a number of reacting, can-type, gas turbine combustors, Moss (1992) measured turbulence levels and calculated the integral length scales. The difference in the combustors was limited mainly to the design of the burner and head region. Overall sizes and appearances were similar for all of the combustor simulators tested. The primary findings indicated good agreement between the measured length scales for the non-reacting versus reacting flows. In addition, measured turbulence levels showed little variance between the non-reacting and reacting simulations. For the combustors simulated, the non-reacting simulations produced maximum turbulence levels of 8.7 and $9.0 \%$, while for the reacting case, the turbulence levels were 9.2 and $9.3 \%$. These finding contradict those found by Goldstein et al. (1983).

\subsection{Studies of Jets in Crossflow}

Flow field measurements pertaining to gas turbine film-cooling were carried out in 1990 by Sinha, Bogard, and Crawford. In particular, the flow was characterized behind two rows of staggered holes, one of which was located 40 hole diameters downstream of the first row (see Figure 2.27). The effects of a thicker boundary layer entering a second row of film-cooling holes were examined. The experiments were conducted for jet-to-mainstream density ratios 1 and 2 . The results showed that the dominant structures appearing downstream of the first row were also present behind the second row. In general, the higher density jets were found to penetrate further into the 
flow due to the momentum flux ratio being twice that of the unit density case. Also affecting the penetration height of the second row of film-cooling holes was the approaching thicker boundary layer created by the first row of film-cooling holes. The jets exiting from the second row were less deflected by the mainstream flow due to a momentum deficiency of the approaching flow at the second row in comparison to the first. The formation of counter-rotating vortices behind the second and first rows was also noted. In addition, turbulence levels were found to be larger for the higher density jets due to an increased momentum flux and jet penetration height into the mainstream. The thicker approaching boundary layer, which increased penetration heights in the second row, also resulted in slightly higher levels of turbulence for the second row of holes as opposed to the first. Lastly, the turbulence field throughout the simulation was found to be highly anisotropic.

In 1997, Sgarzi and Leboeuf numerically investigated the different vortical structures present in the flow generated by a jet in crossflow. The computations were performed using a stationary three-dimensional Navier-Stokes code. Up to five vortices were identified and are shown in Figure 2.28. Amongst all of the vortices, the counterrotating vortices were found to be the most dominant in the downstream flow. The counter-rotating vortices resulted from the stretching and warping of the annular vorticity rings issuing from the injection pipe. Two vortices were noted on the upstream side of the jet. The first was the horse-shoe vortex, which was typical of the near-wall effects due to the blockage created by the jet in the mainstream. Specifically, the horse-shoe vortex resulted from the strong deceleration of the crossflow boundary layer as it moved towards the jet. It was convected to the low pressure region on the downstream side of the jet. A lip vortex was found upstream the jet boundary as a consequence of the complex flow topology near the jet boundary that included a pair of stagnation points. Two other wake vortices were also noted as a consequence of the crossflow boundary layer. The crossflow boundary layer was either entrained into the jet, inducing the halfwake vortex pair, or swept by the counter-rotating vortices, creating the half-wall vortex pair. The half-wall vortex pair prevented the jet from sweeping the wall, and could possibly have detrimental effects on the protection of the wall in this region. For jets injected at an angle less than $90^{\circ}$ with respect to the wall, all of the vortical structures 
were pushed towards the wall, and for some cases, the half-wall vortex pair may disappear. This comprehensive study was one of the first to qualify the wake vortices produced by the crossflow boundary layer.

Rydholm (1998) constructed and tested a combustor model of the liner cooling that fulfilled all of the scaling laws (Reynolds, Mach and Prandtl numbers). The experimental conditions were chosen to reflect those of a real effusion cooled (filmcooling by discrete holes) combustion chamber. The model consisted of one transverse row of film-cooling holes with a thirty-degree angle to the wall through which cooling air was injected into a cross flowing mainstream (Figure 2.29); note, in actual combustor liners there are numerous rows of film-cooling holes. The experiments were carried out for varying density ratios between the cooling flow and the mainstream, as well as slightly differing low momentum fluxes for the film-cooling holes. The experimental results for these small-scale film-cooling jets showed a strong three-dimensional flow field with two counter-rotating vortices on either sides of the jet. These vortices were shown to create a downwash of high-speed fluid to the wall, which transported the lowspeed fluid from the wall into the mainstream leaving behind a velocity deficit wake surrounded by a highly turbulent shear layer. An increase in the momentum flux was shown to increase the strengths of these vortices, as well as the distance between the wake and the wall. An increase in the density ratio proved to decrease the velocity gradients, which ultimately resulted in lower turbulence levels. Furthermore, measurements showed that the turbulence near the hole was highly anisotropic, and while higher momentum fluxes only increased the turbulence levels, the level of anisotropicity remained the same. Lastly, as the jet proceeded downstream, an increase in the distance between the velocity deficit wake and the wall was observed; however, the same distance with regards to the cool core of the temperature measurements hardly varied.

\subsection{Summary of Current Literature}

Many of the findings here concentrated on can-type combustors with the exception of a few annular test rigs. For the most part, a number of the non-reacting 
experimental simulators were not full combustor simulators. Many of them focused on one particular aspect of the combustor; for example, jets in crossflow, such as filmcooling and dilution rows, or swirler effects. In all, the literature indicates that the profiles exiting the combustor are highly non-uniform in pressure, velocity, and most notably temperature. The initiators of these non-uniformities were primarily due to the dilution flow within the combustor and on a smaller scale, the film-cooling holes or slots. These dilution jets were also found to considerably contribute to turbulence levels within the combustor simulators. Computational results proved to be extremely valuable in developing a reasonably accurate combustor. They provided large savings in money and time. However, in general, the computational trade-off between time and accuracy often led to gross under predictions in the mixing and turbulence levels particularly downstream of dilution jets and film-cooling.

Halls (1970) and Moss (1992) presented experimental results for reacting combustors. The primary findings were that characteristics such as the integral length scales, turbulence levels, and exit temperature profiles remained the same with and without reaction. These findings are crucial in validating the accuracy and reality of nonreacting simulations over more costly, complex, and time-consuming reacting rigs and simulations.

To date, there is still insufficient data as far as the temperature and flow fields within a full three-dimensional annular combustor model. In particular, hardly any threecomponent velocity measurements are presented. Furthermore, corresponding temperature planes within the combustor are few in number, and combined velocity and temperature fields are virtually non-existent. Turbulence measurements within the central core of the combustor are also lacking. As stated earlier, many of the nonreacting combustor studies focus only on one or two particular aspects of combustor flow. For example, Holdeman (1993) studied only the dilution jets; Stevens and Carotte (1990) examined dilution injection on only one side of the combustor.

Studies by Sinha et al. (1990) and Sgarzi and Lebouf (1997) qualified the vortical structures appearing around film-cooling injection. However, to date, few studies have been performed to identify the vortical characteristics appearing in and around large dilution jets coupled with film-cooling. 
The uniqueness of the combustor simulator used in my study is that it is a complete linear representation of a true Pratt and Whitney engine. The simulator combines the effect of two rows of streamwise staggered and spanwise aligned dilution jets with film-cooling completely along the combustor liner walls; all of this is upstream of a stator vane sector. Furthermore, the flow conditions applied in this study are representative of an actual aircraft engine. The current literature does not include any data on the temperature and flow field distribution for high momentum flux dilution jets combined with low momentum flux liner film-cooling. In addition, the current literature fails to address the effects behind two rows of dilution jets. In all, a more complete experimental understanding is needed to help engineers in determining how to efficiently and cost-effectively design gas turbine combustors. 


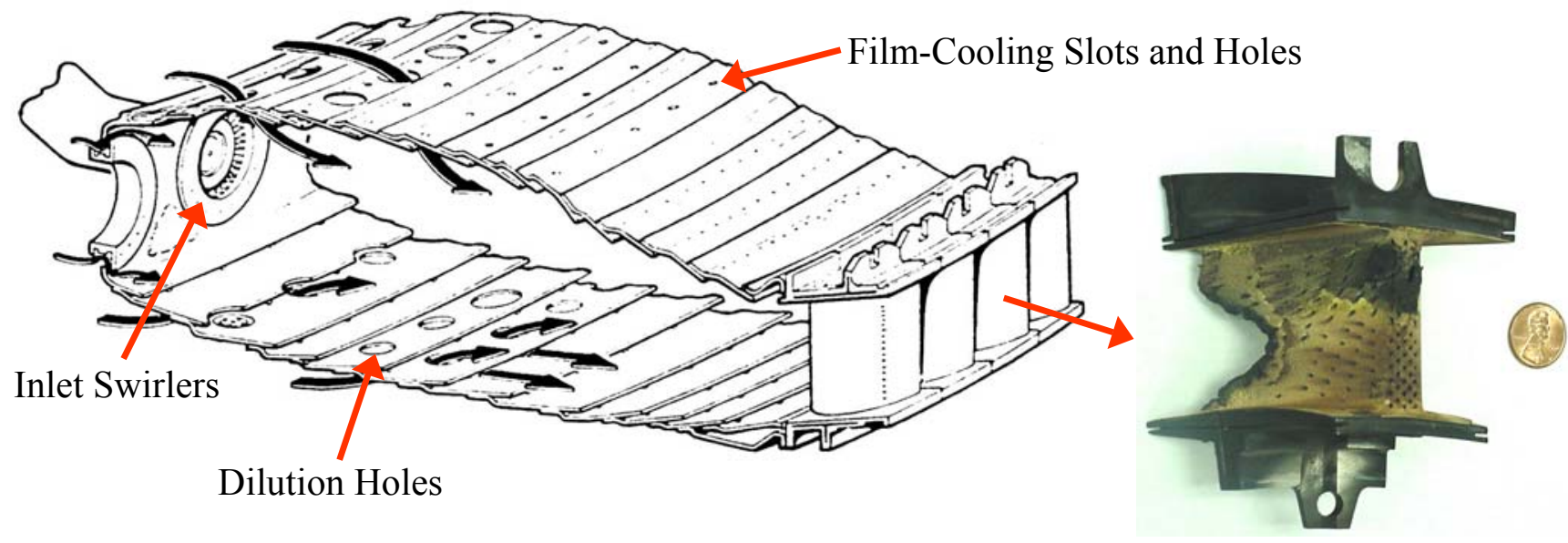

Figure 2.1 Diagram of a typical annular combustor (Pratt \& Whitney, 1988) and the damage that can occur to a first stage turbine vane.
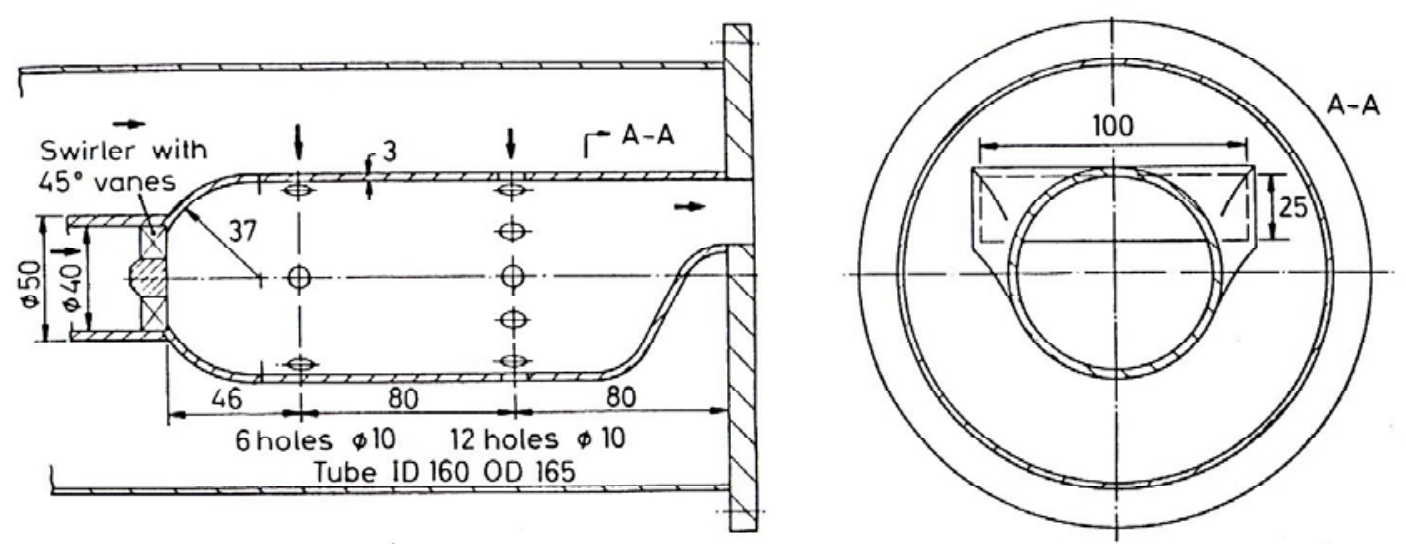

Figure 2.2 Schematic of the combustor simulator used by Koutmos and McGuirk (1989). 


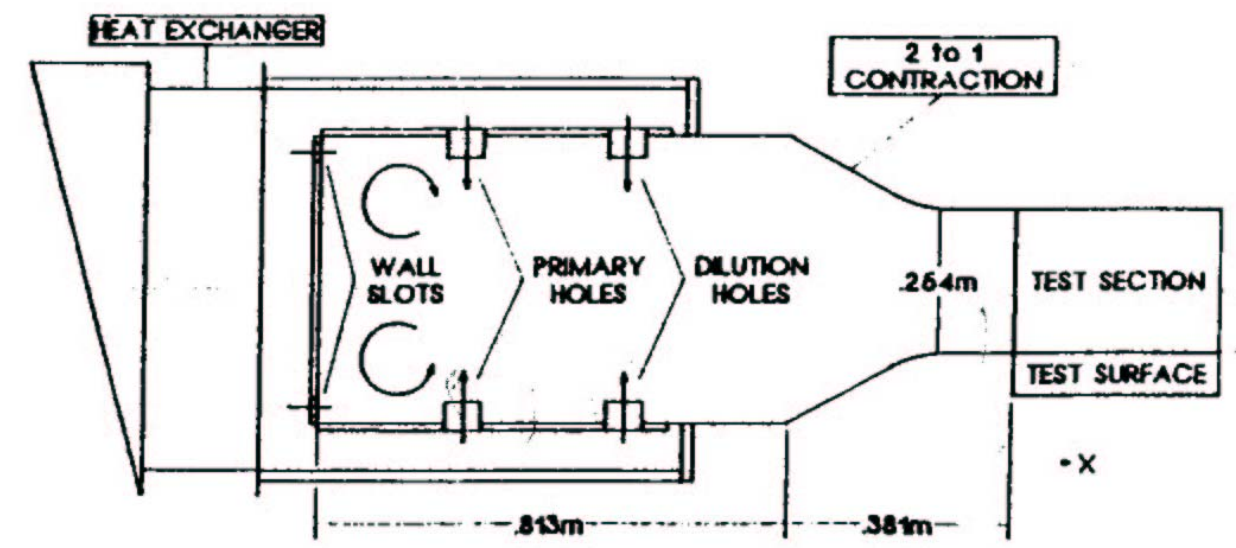

Side view of turbulence generator, geometry $* 2$

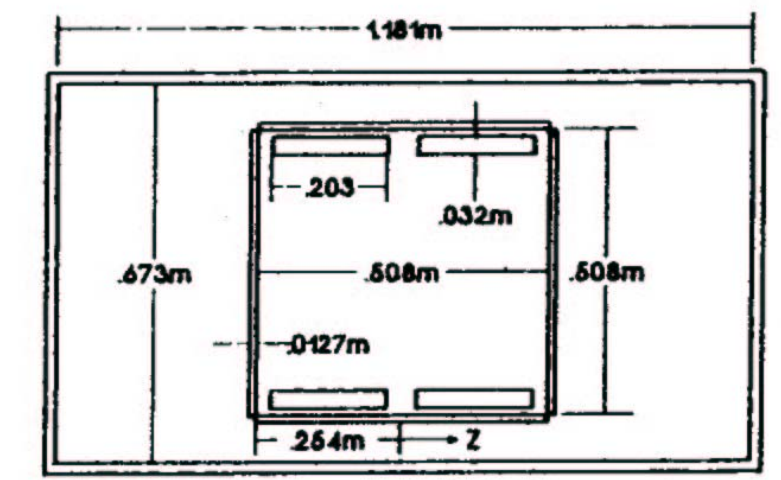

SIMULATOR END PANE AND PRESSURE BOX CROSS-SEC MONAL VIEW (BACK)

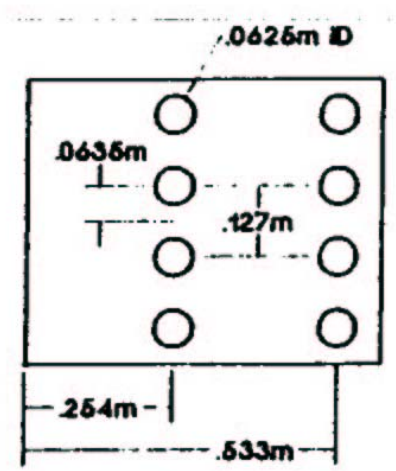

SIMUL.ATOR UNER PANE TOP \& BOTTOM)

Back view of turbulence generator and liner panel, geometry $\bullet 2$

Figure 2.3 Schematic of combustor simulator used by Ames and Moffat (1990). 


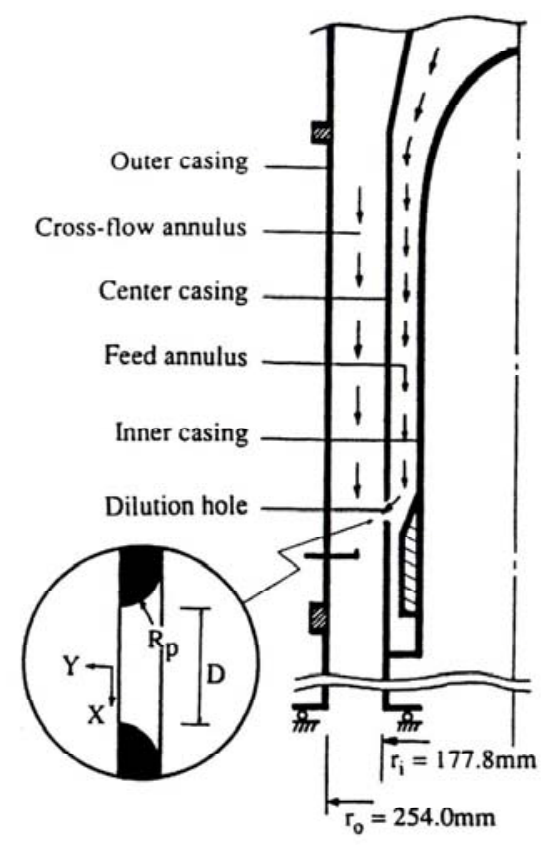

Figure 2.4 Schematic of Stevens and Carotte (1990) test facility.

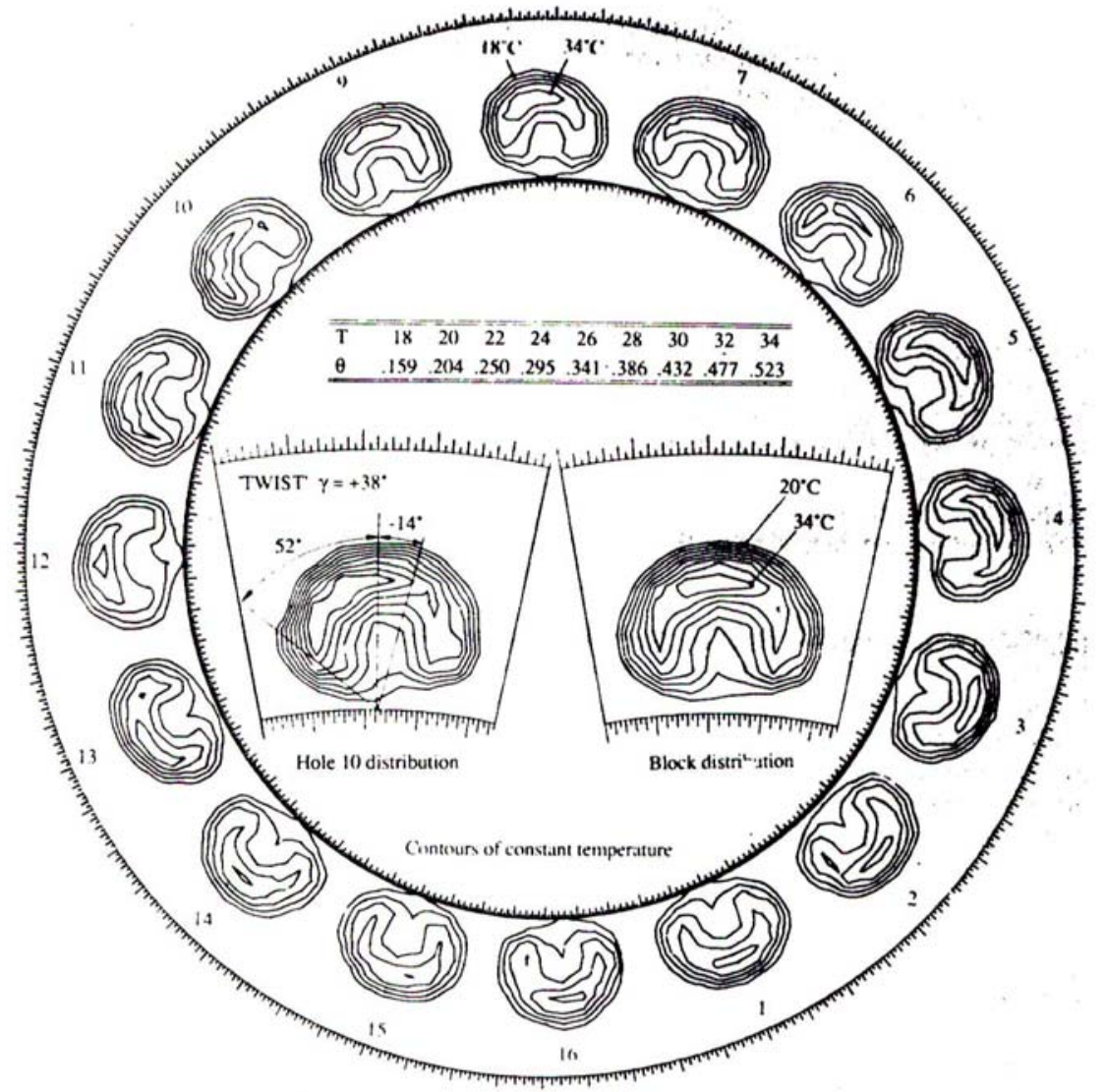

Figure 2.5 Graph of the temperature measurements by Stevens and Carotte (1990) showing the kidney-shaped profiles. 


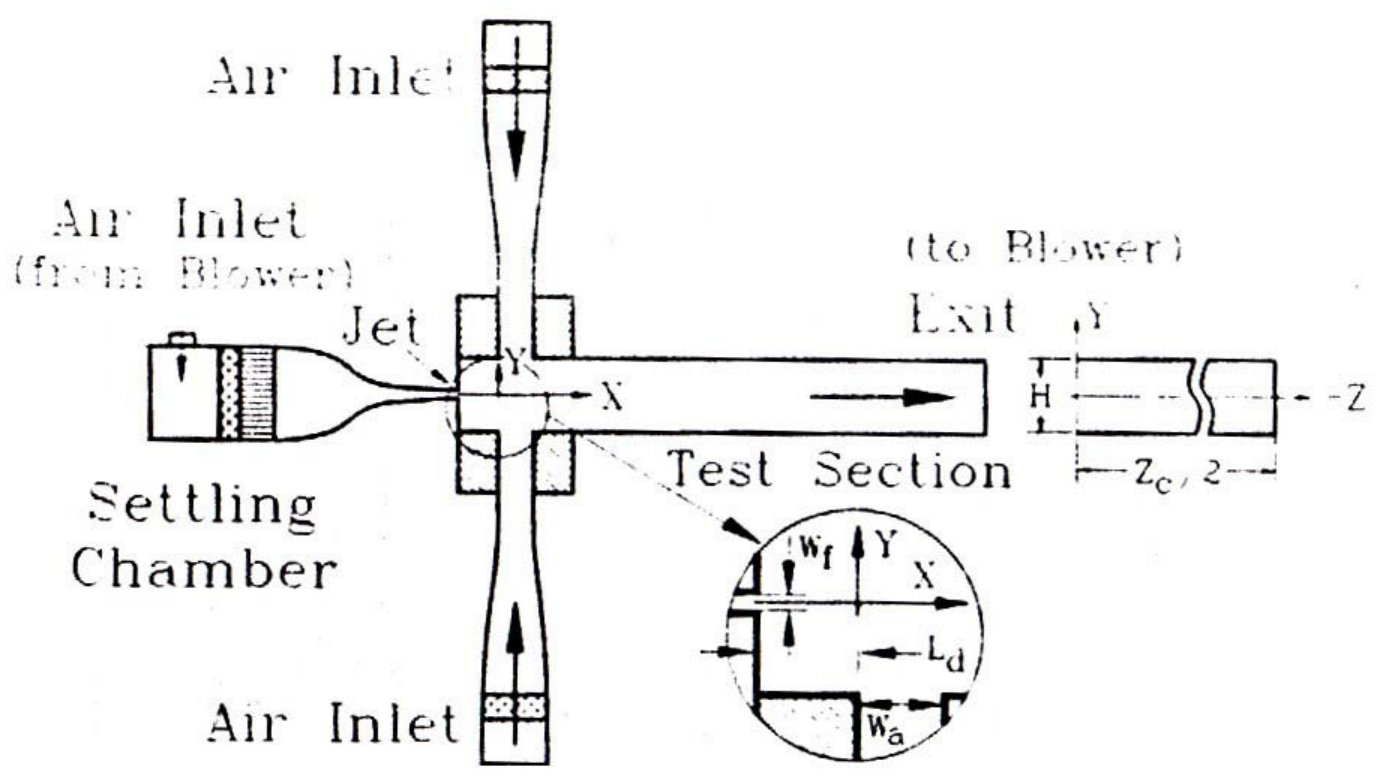

Figure 2.6 Test section used by Liou and Wu (1992). 
$\varnothing 8$
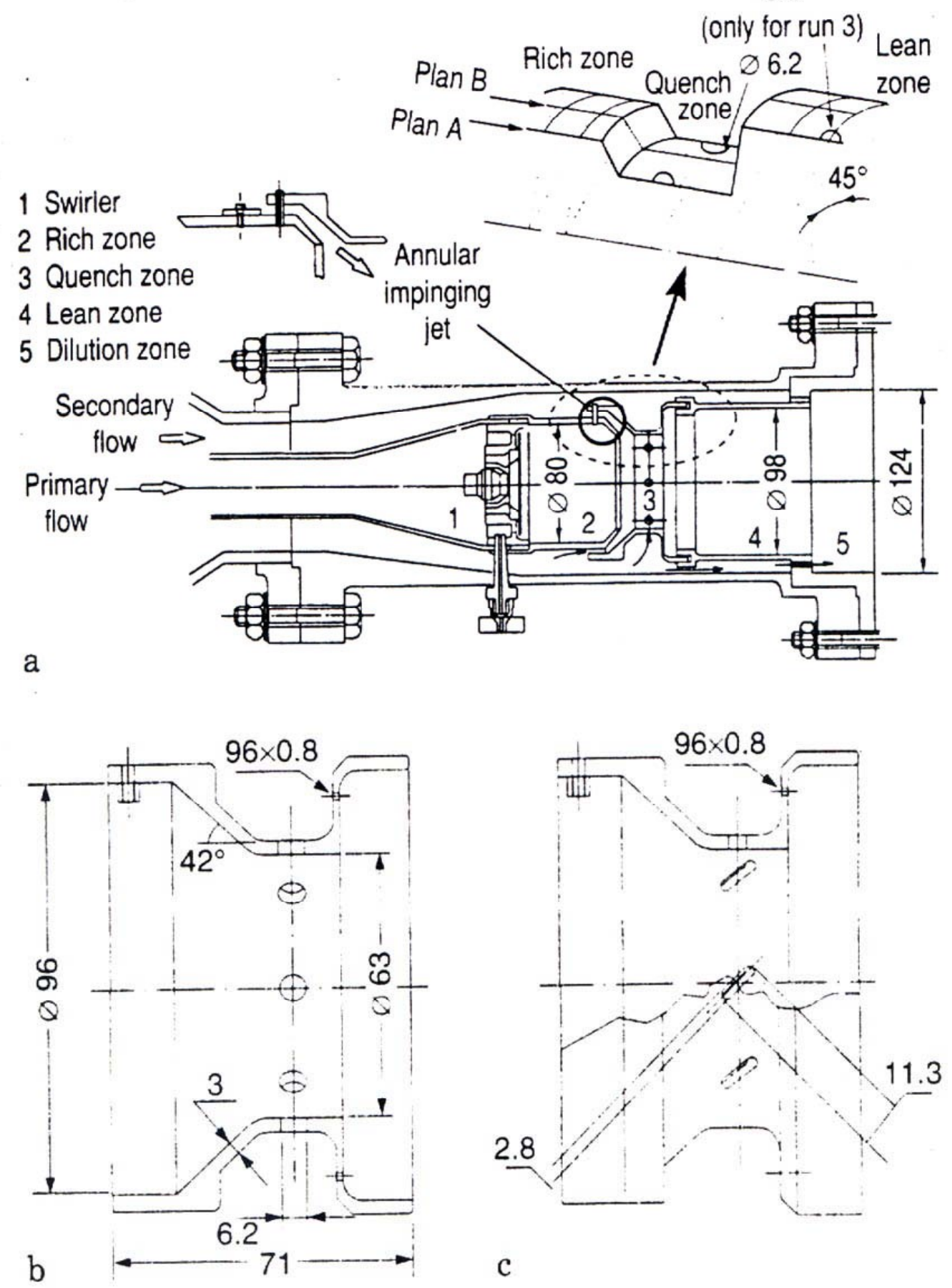

Figure 2.7 Schematic showing Anacleto, Heitor, and Moreira (1996) combustor model. 


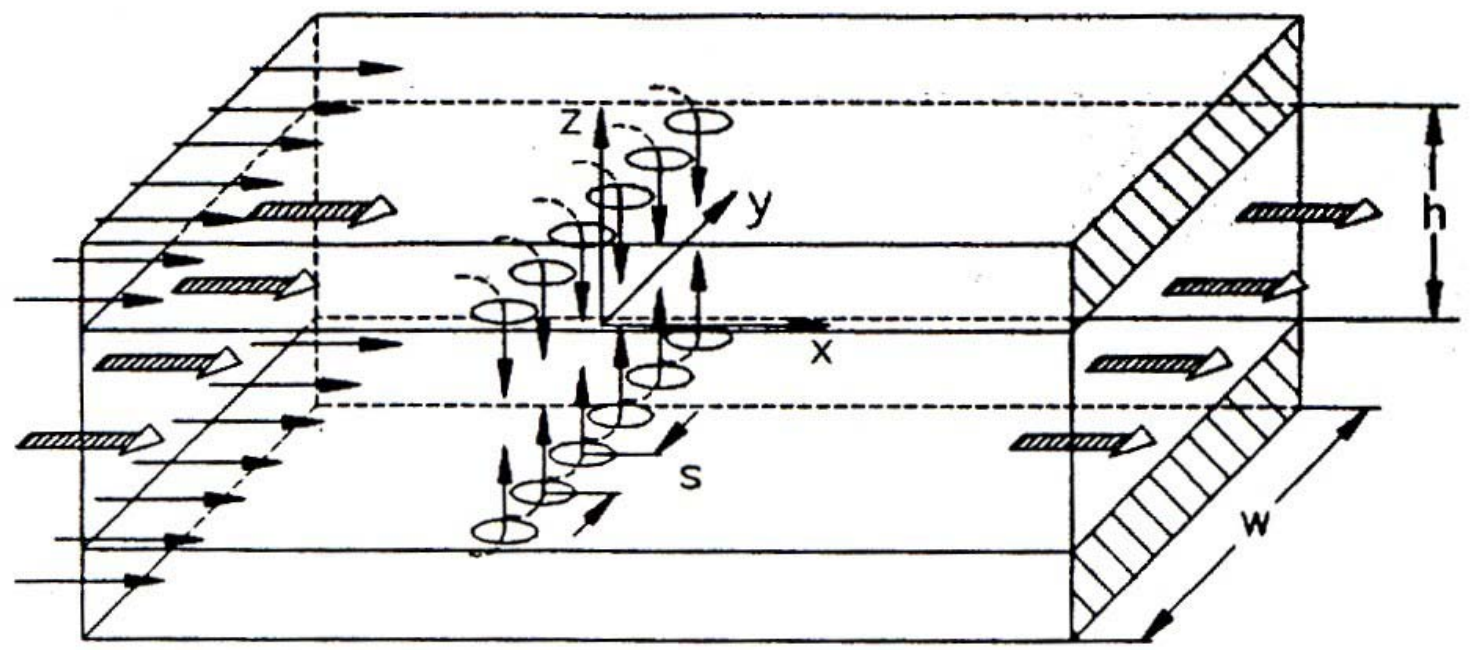

Figure 2.8 Schematic of Doerr, Blomeyer, and Hennecke (1997) combustor model.

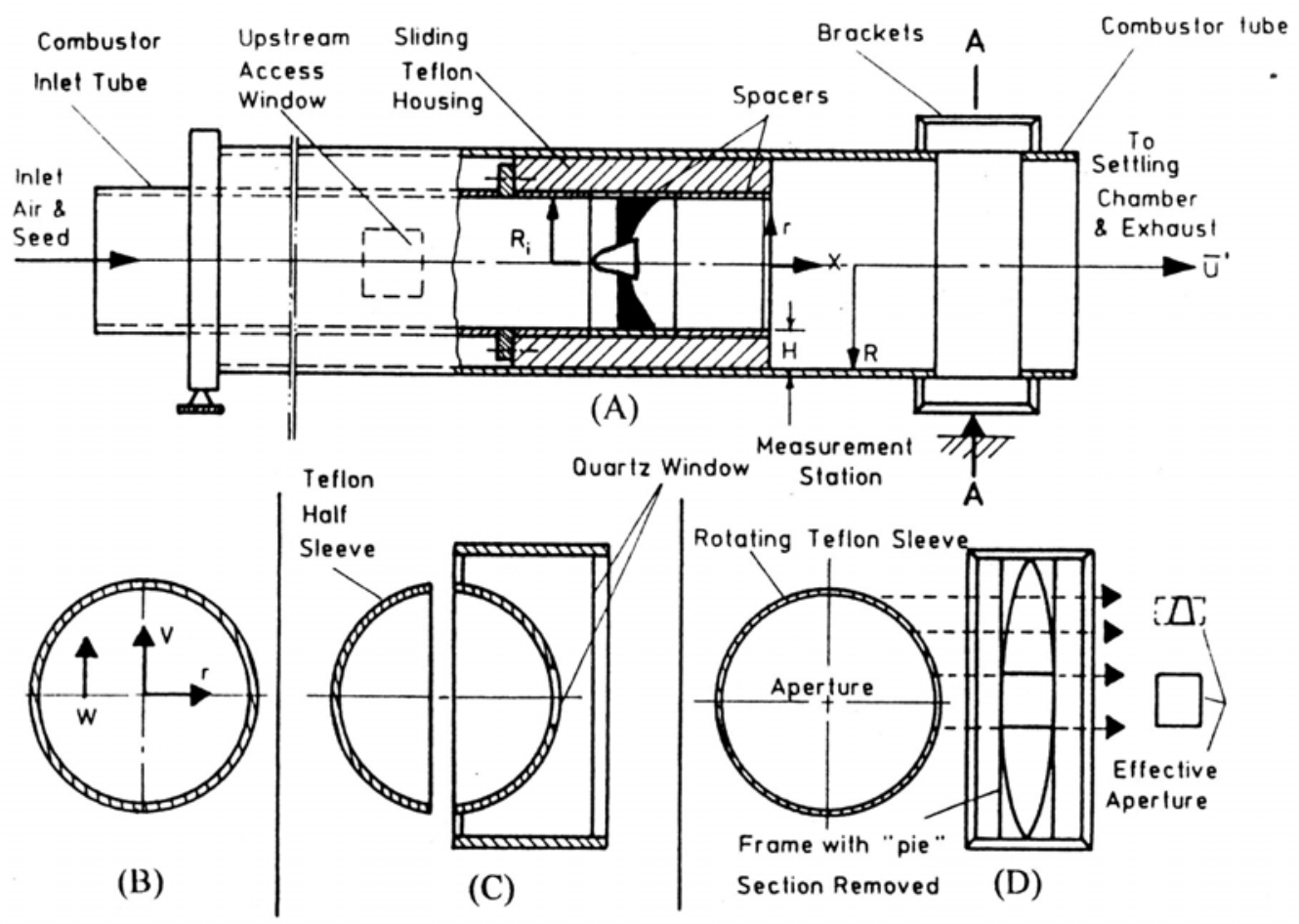

Figure 2.9 Ahmed (1998) combustor simulator model. 


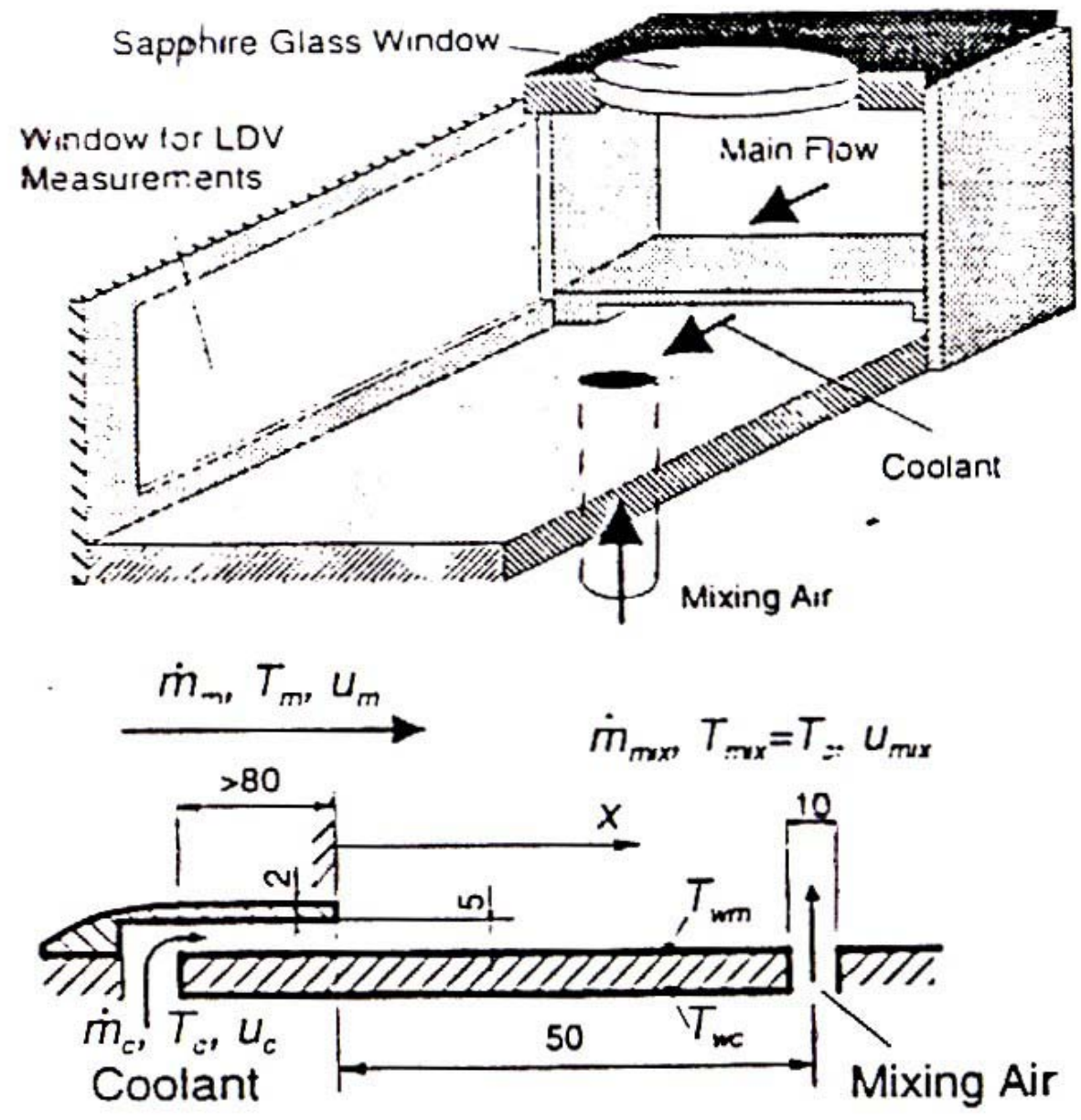

Figure 2.10 Schematic showing Gritsch, Martiny, Schulz, Kim, and Wittig (1998) combustor simulator model.

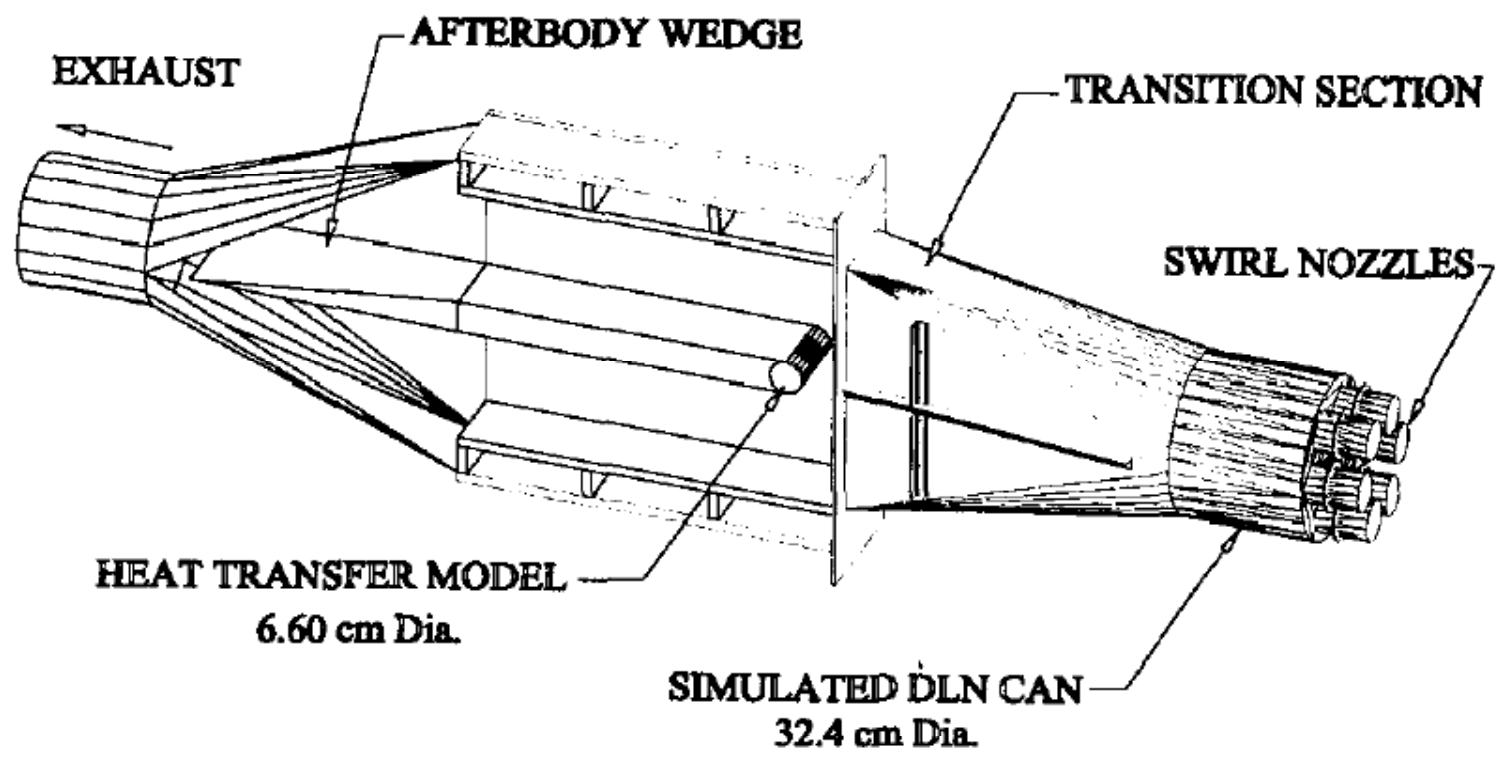

Figure 2.11 Schematic of simulator used by Van Fossen and Bunker (2001). 


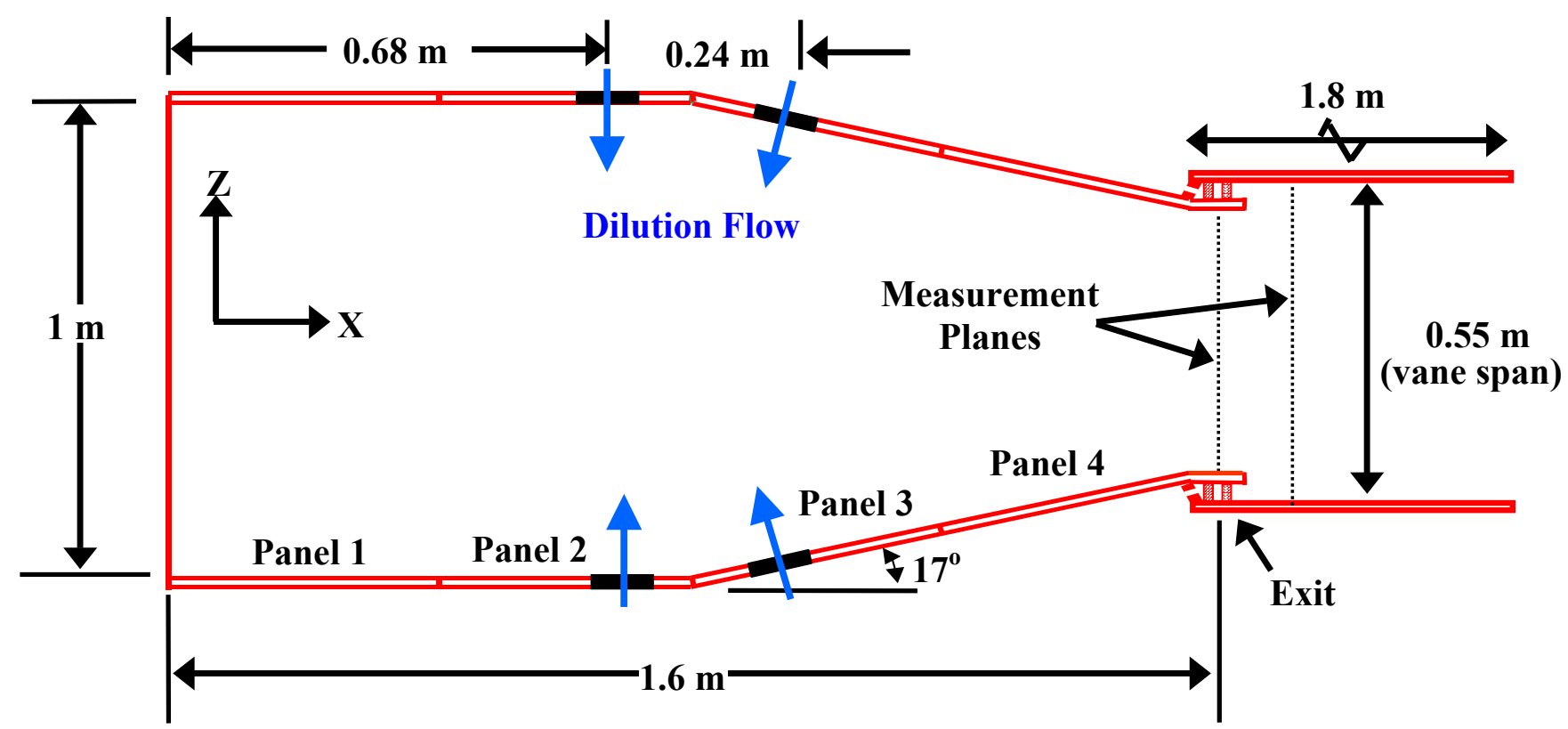

Figure 2.12 Schematic side view of the combustor simulator used by Barringer, Richard, Walter, Stitzel, and Thole (2002).
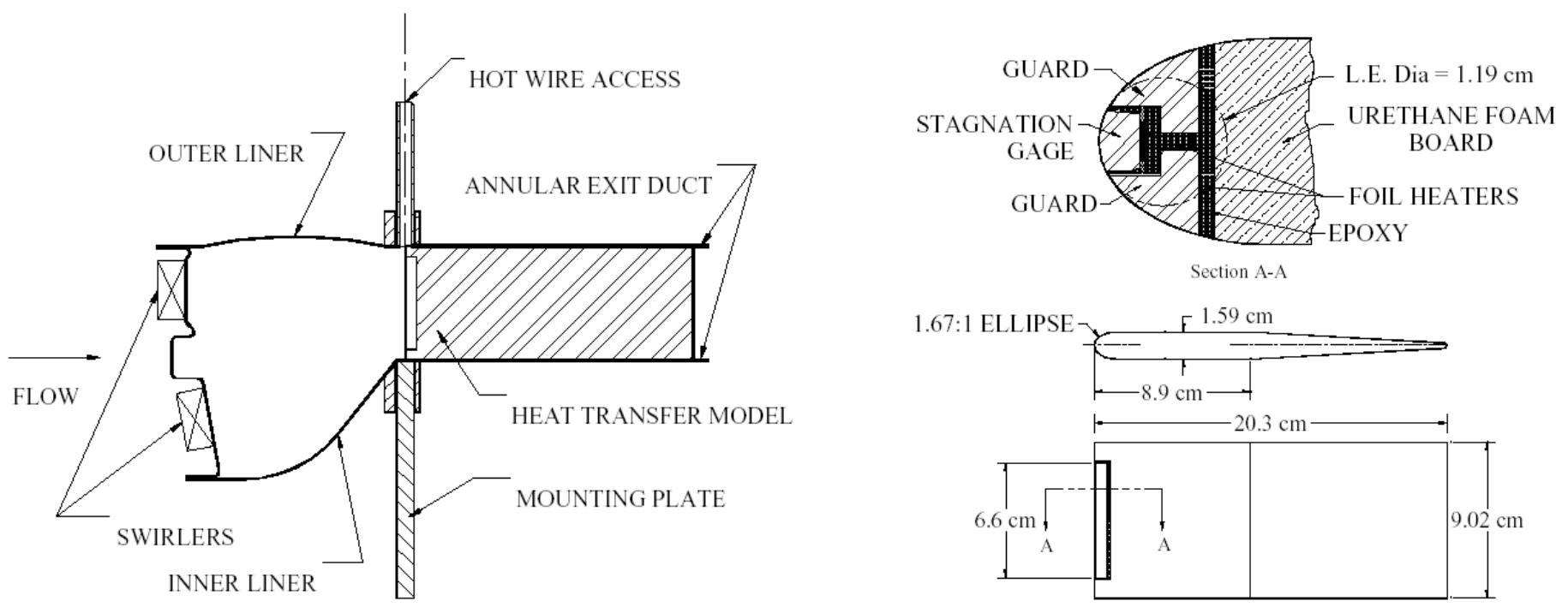

Figure 2.13 Schematic view of the combustor rig used by Van Fossen and Bunker (2002). On the right is the heat transfer model and on the left is a crosssectional view of the rig. 


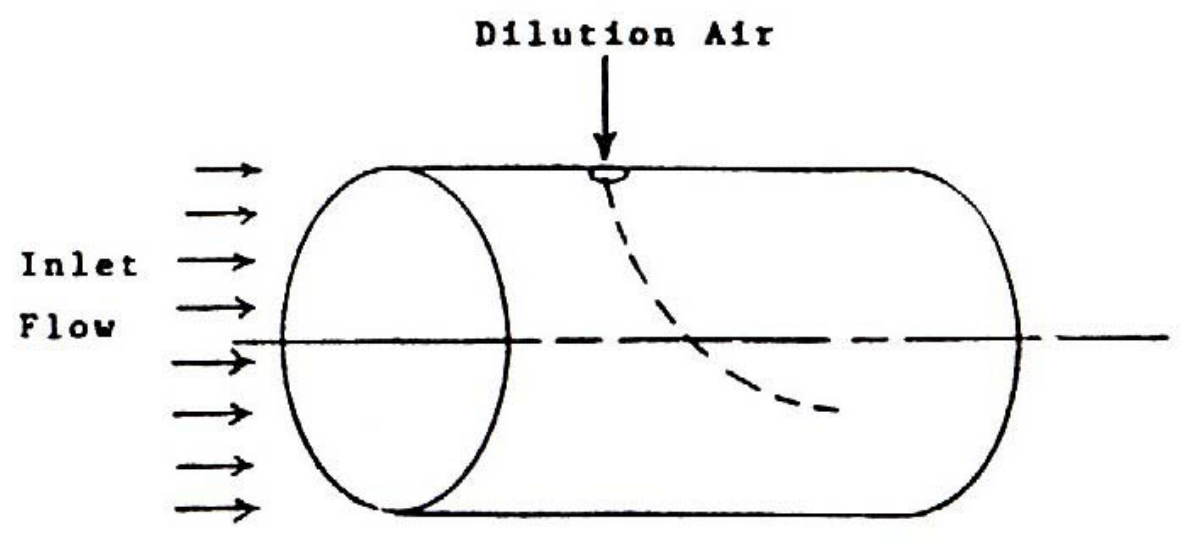

Figure 2.14 Schematic of Busnaina (1987) combustor simulator modeled by CFD. 

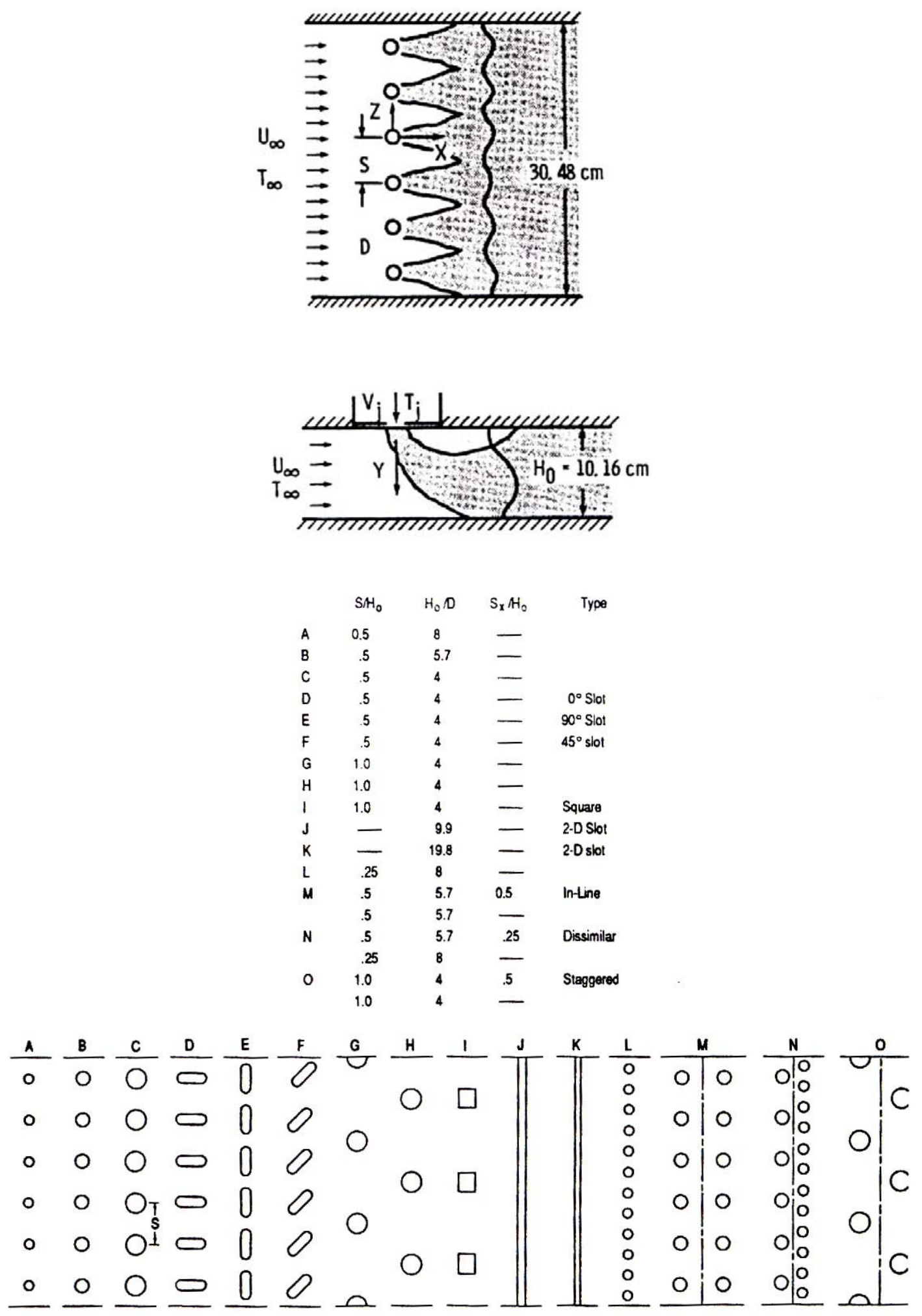

Figure 2.15 Schematic of Holdeman (1993) combustor simulator with different hole shapes, orientations, and patterns tested appearing below. 

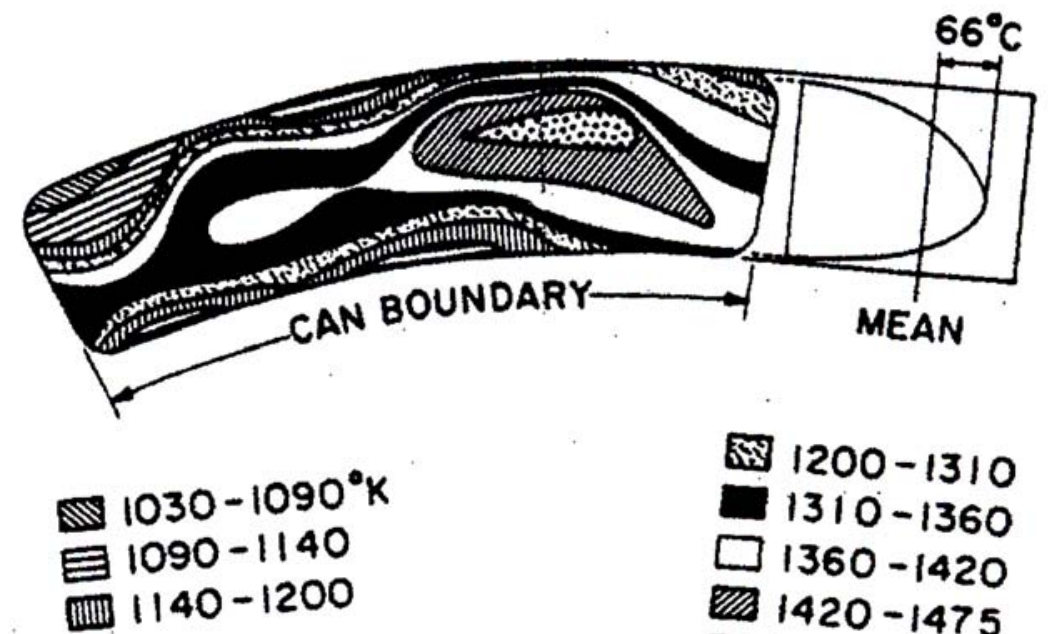

$1200-1310$

$1310-1360$

$\square 1360-1420$

$1420-1475$

$1475-1510$

Figure 2.16 Contours of the non-uniform temperature profile measured at the exit of a reacting combustor presented by Halls (1970).
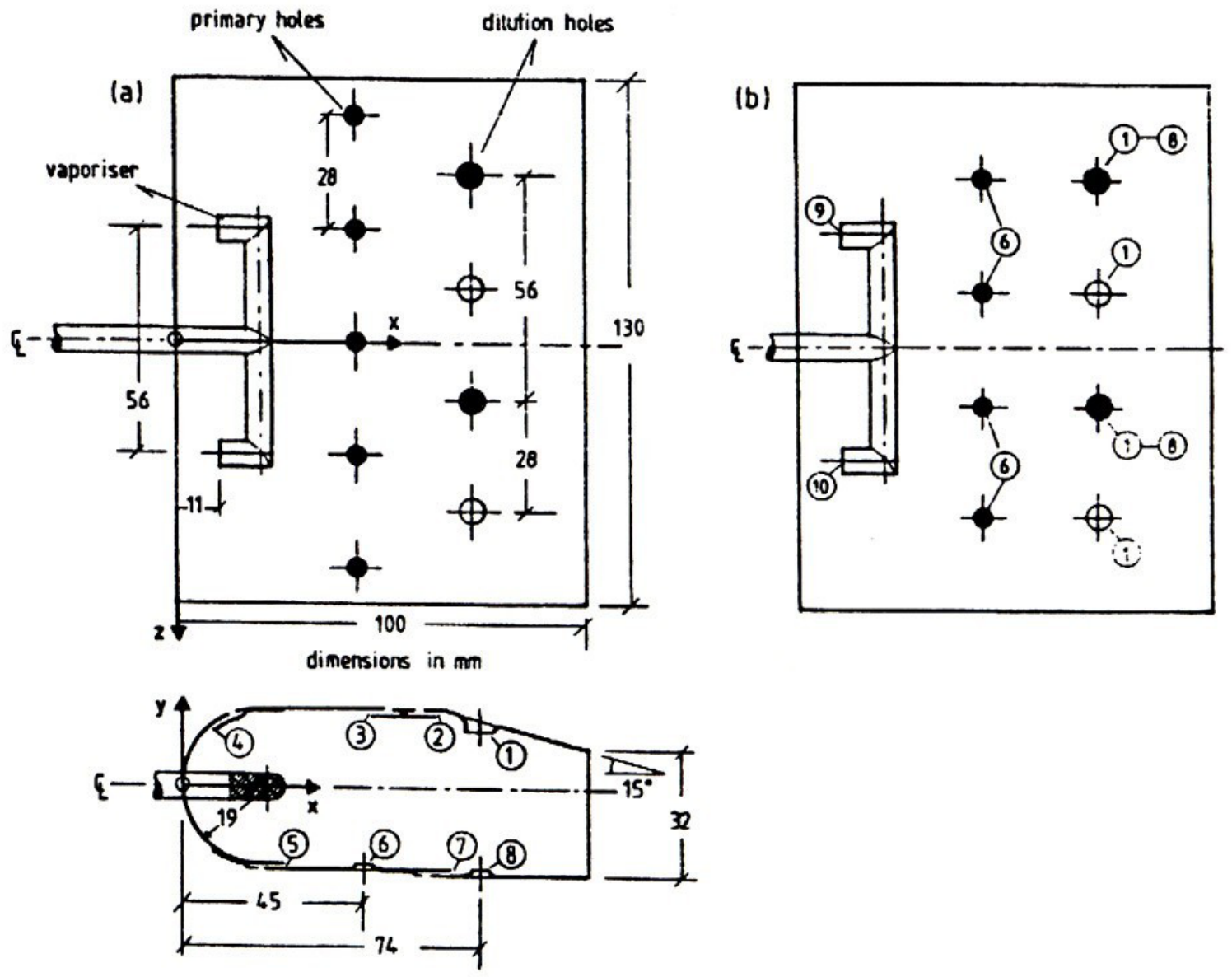

Combustor geometries: (a) Combustor 1; (b) Combustor 2 .

Figure 2.17 Schematic of Bicen, Tse, and Whitelaw (1988) combustor model. 

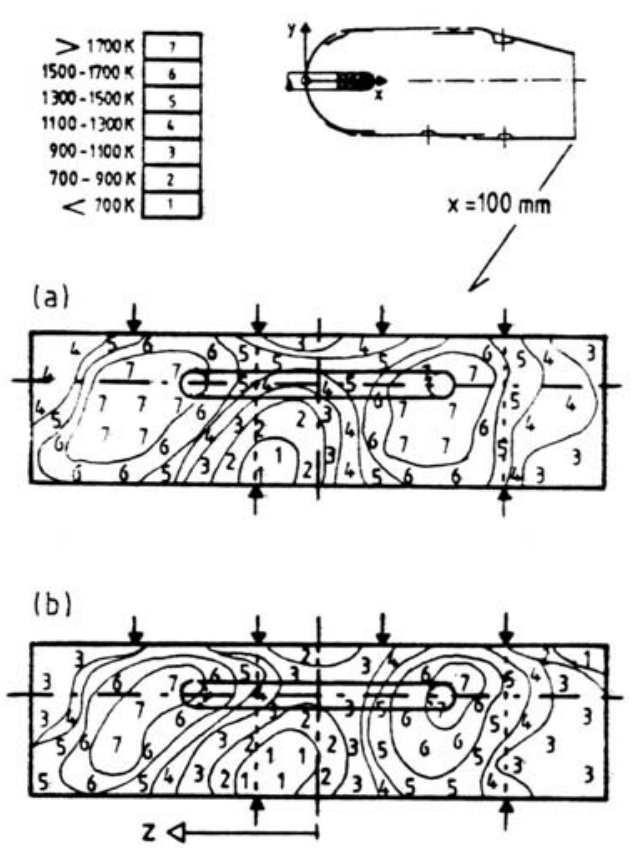

Exit-plane distributions of mean temperature with Combustor 1: (a) air-fuel ratio of 29; (b) air-fuel ratio of 37 .

(a)
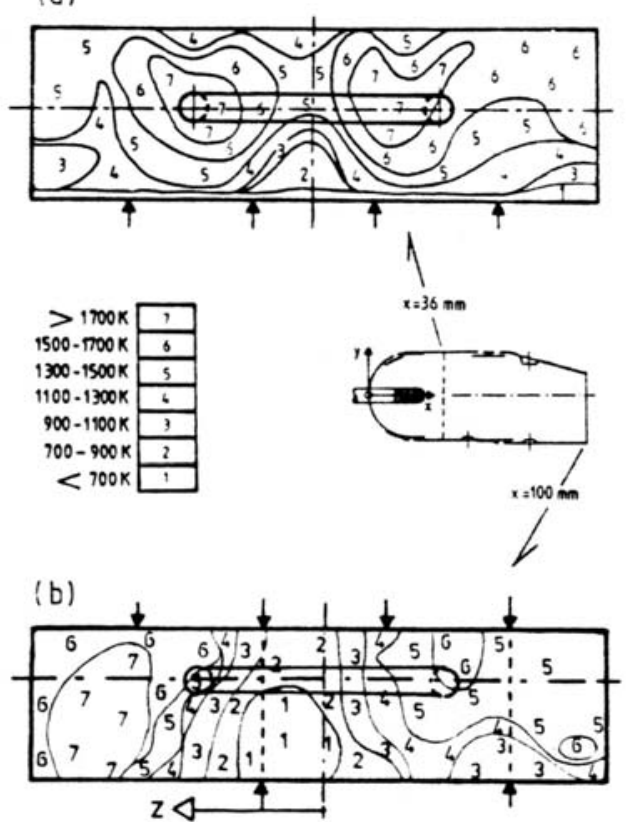

Cross-plane distributions of mean temperature with Combustor 2 and air-fuel ratio of 37: (a) primary zone: (b) exit plane.
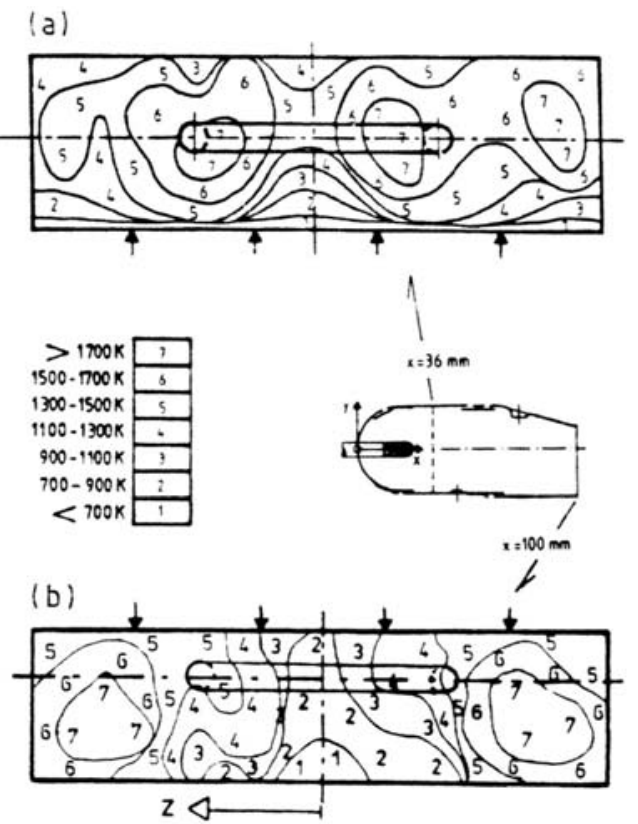

Cross-plane distributions of mean temperature with lower dilution holes of Combustor 2 blocked and air-fuel ratio of 37: (a) primary zone; (b) exit plane.

Figure 2.18 Contours of the temperature results obtained by Bicen, Tse, and Whitelaw (1988). 


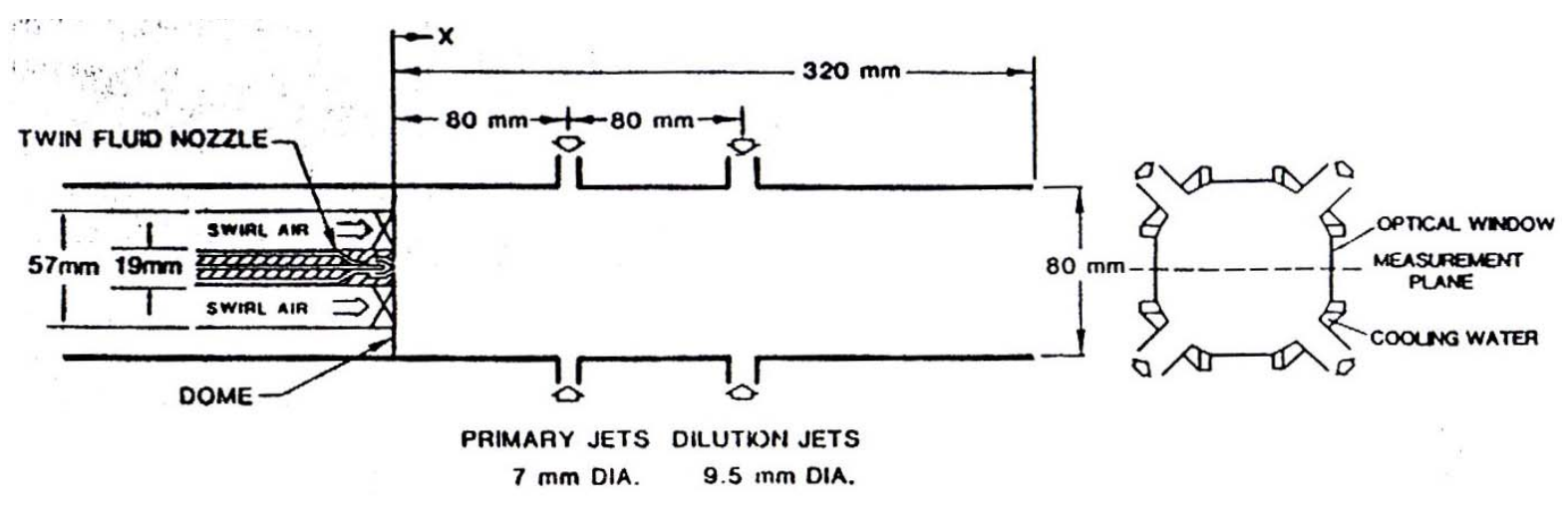

b) Module 1 .

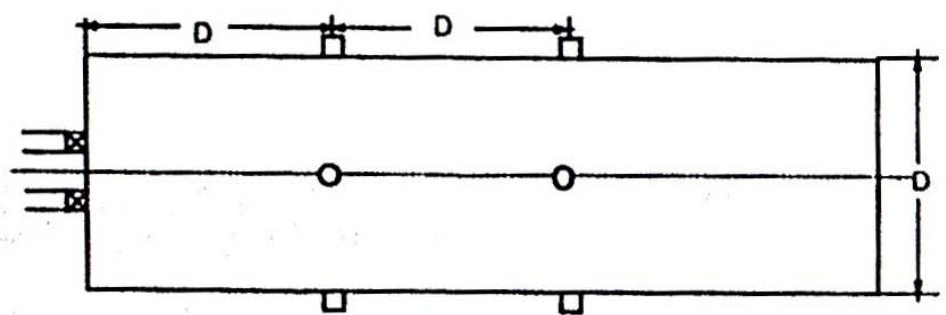

c) Module 2 .

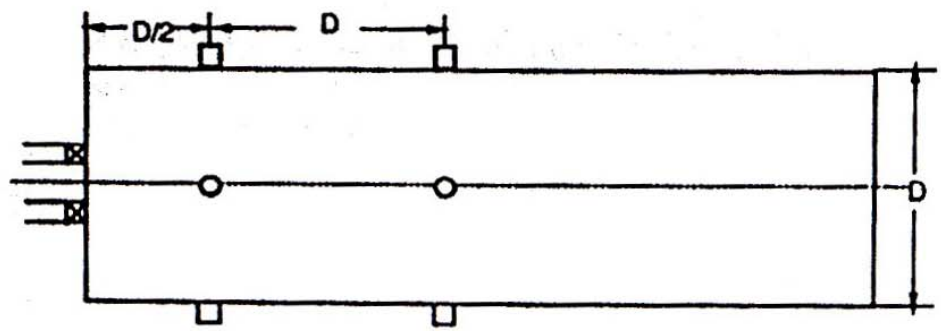

d) Module 3 .

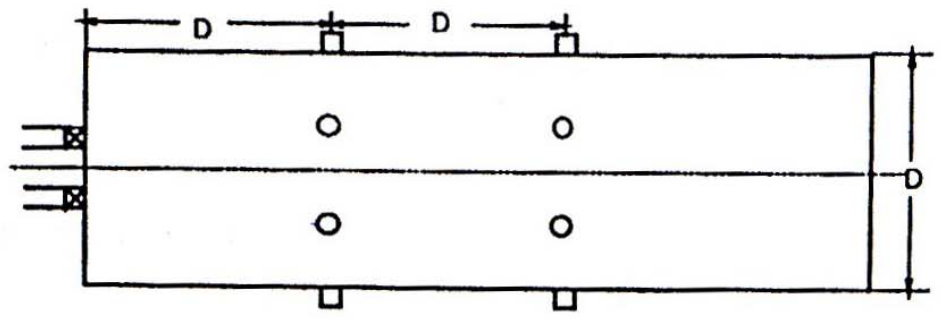

Figure 2.19 Schematic of the reacting combustor model used by Richardson and Samuelson (1992). 


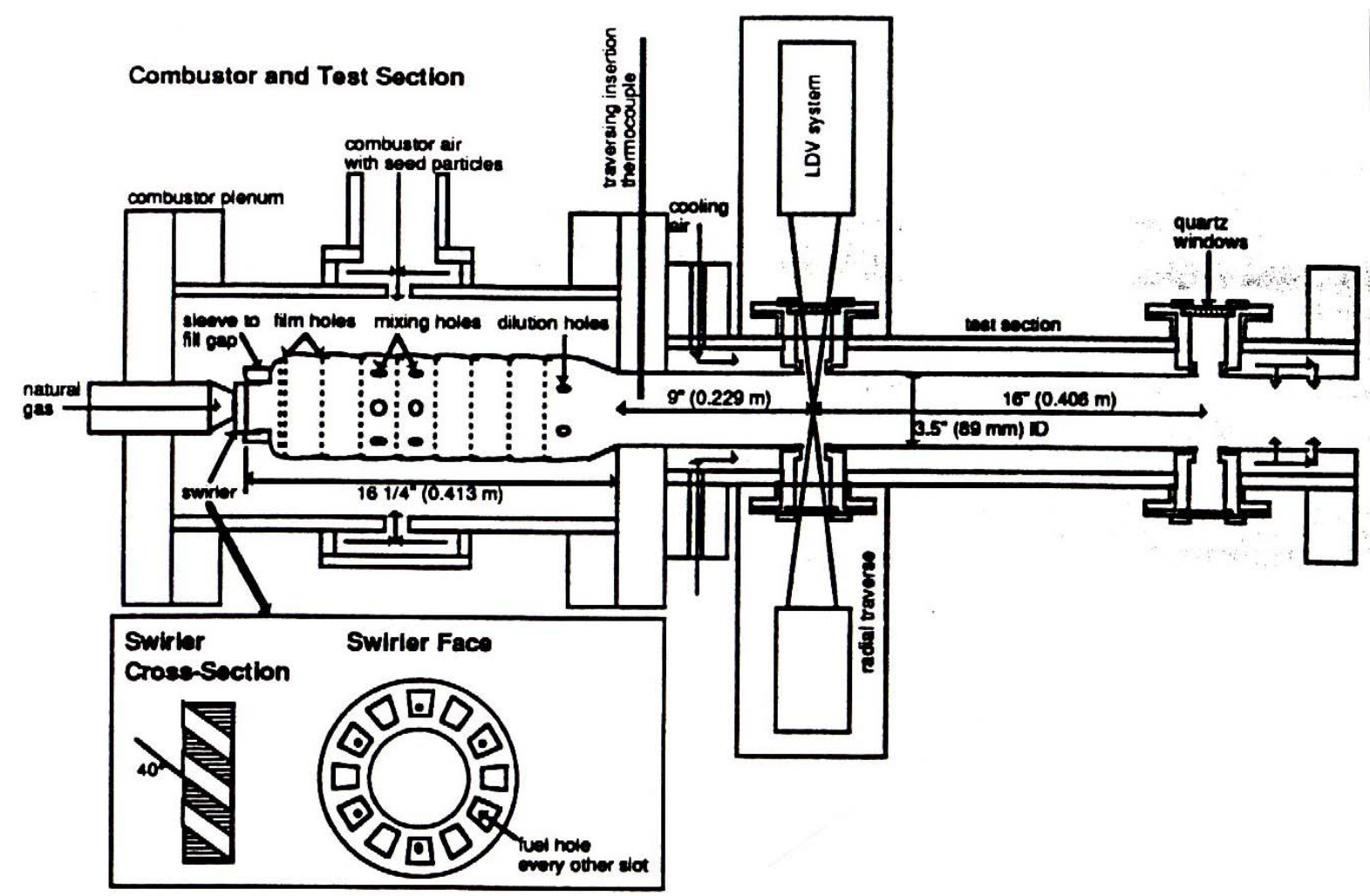

Figure 2.20 Schematic of Goebel, Abauf, Lovett, and Lee (1993) combustor simulator.

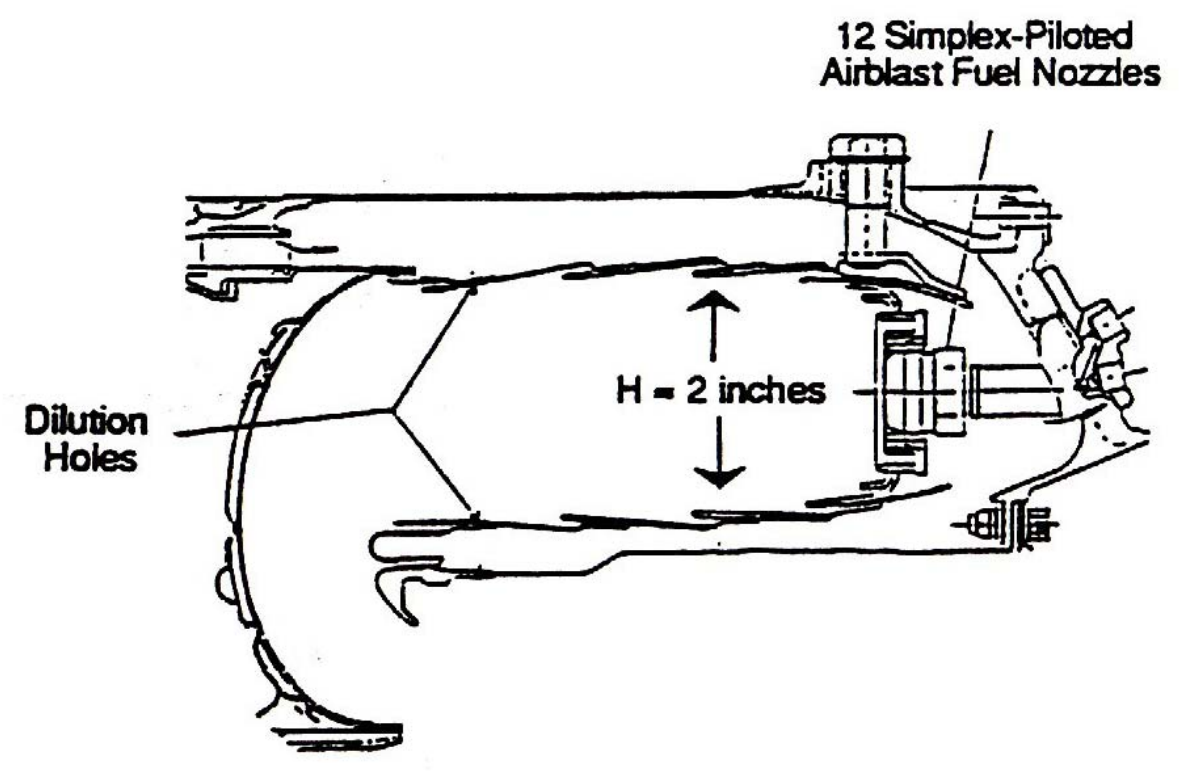

Figure 2.21 Crocker, Smith, and Myers (1994) reacting Allied Signal F109 combustor. 


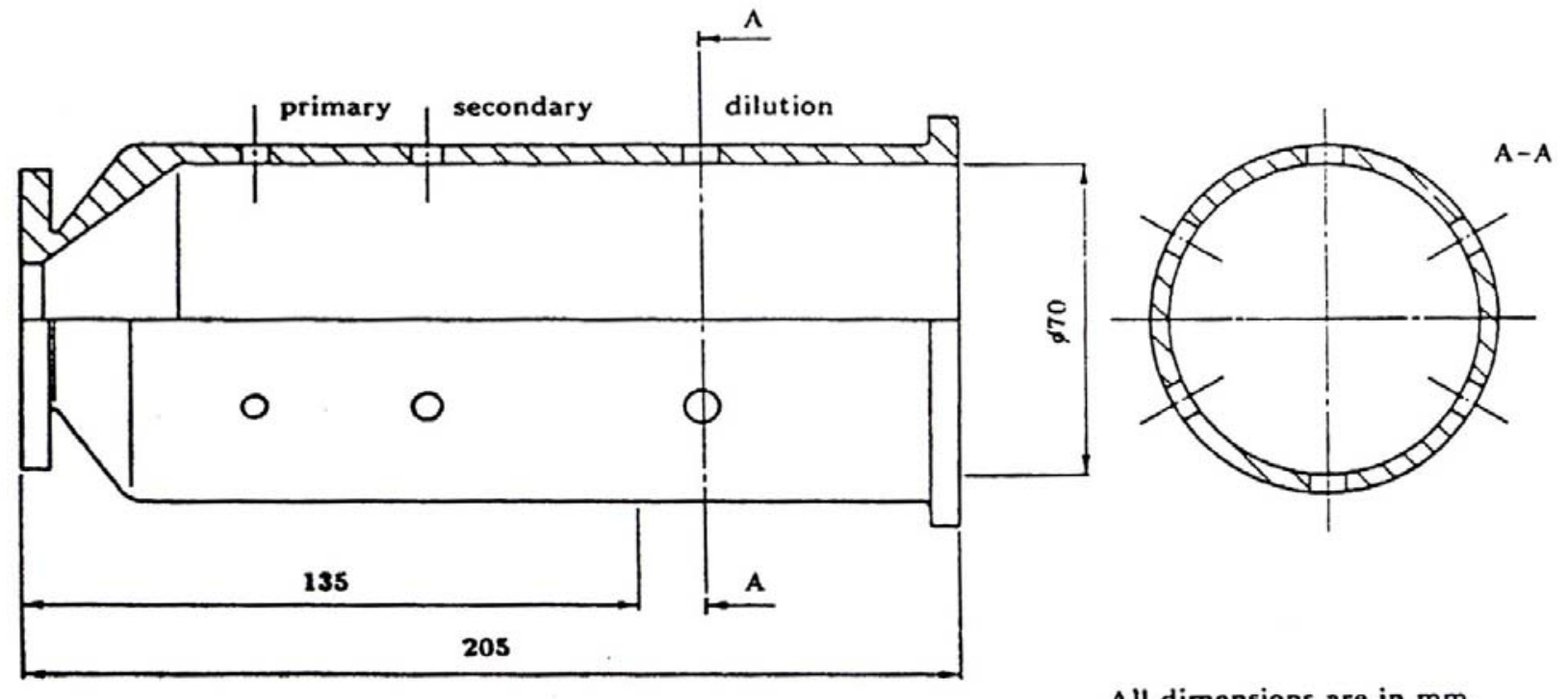

All dimensions are in $\mathrm{mm}$.

Figure 2.22 Schematic of the can combustor modeled by Ebbinghaus and Swithenbank (1995).

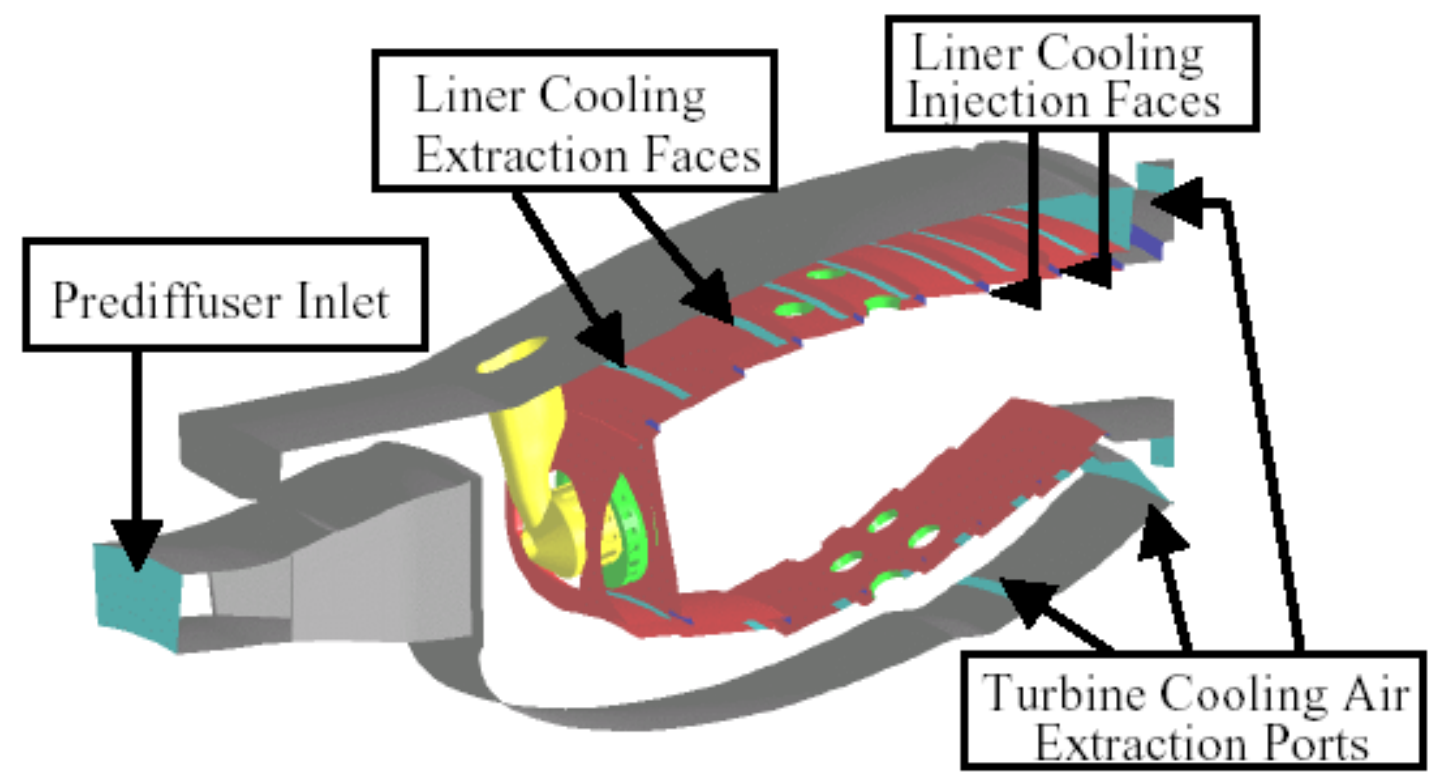

Figure 2.23 Schematic of PW4098 combustor model created for validation of the newly created CFD system by Malecki et al. (2001). 

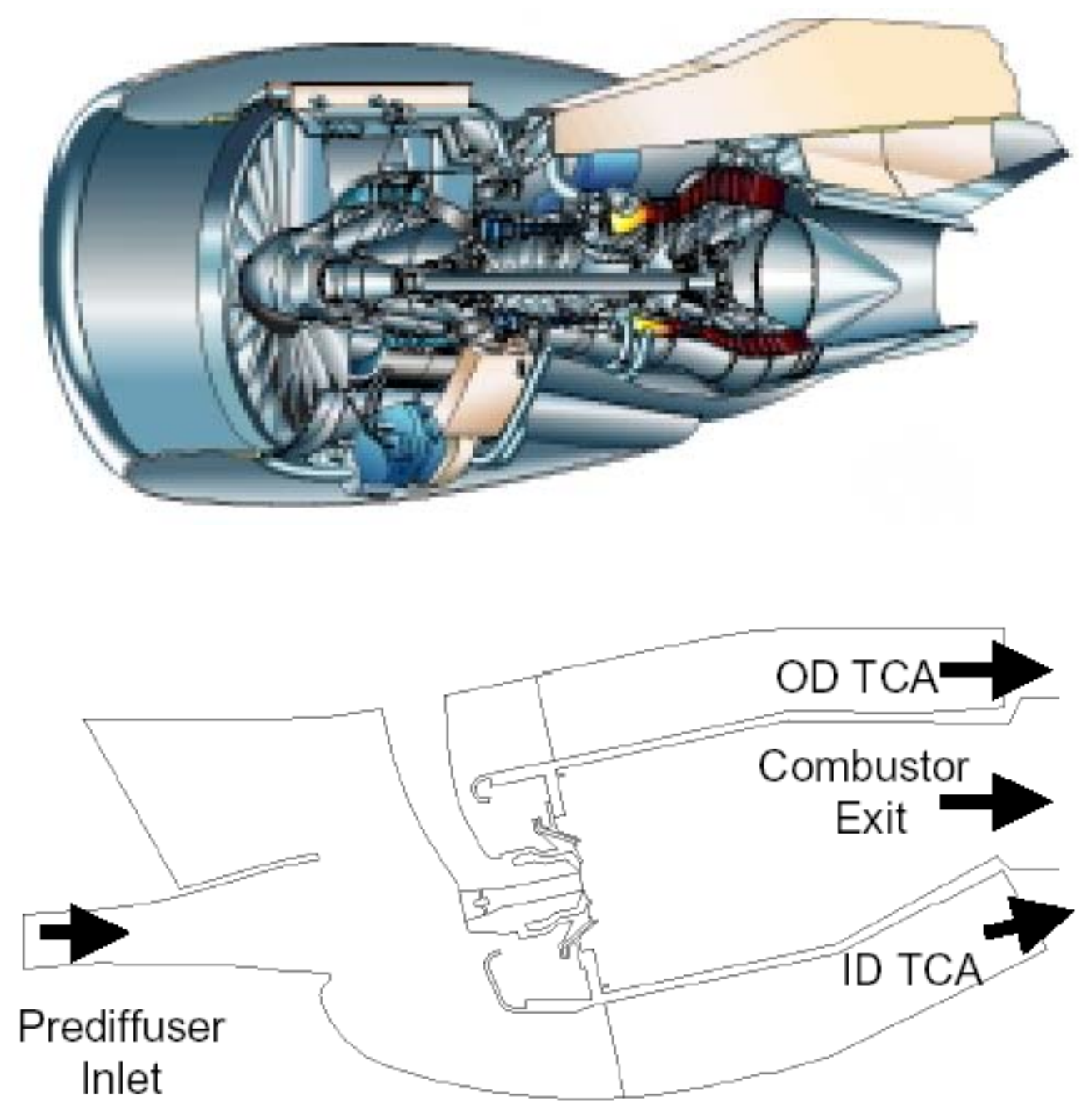

Figure 2.24 Above, a picture of the PW6000 engine and below, its computational combustor model. This study used the CFD system developed by Malecki et al. (2001) to design the PW6000. Following its design, a full-scale operating test rig was constructed and the computational results were validated against experimental measurements. All design and analysis on the PW6000 was done by Snyder et al. (2001). 

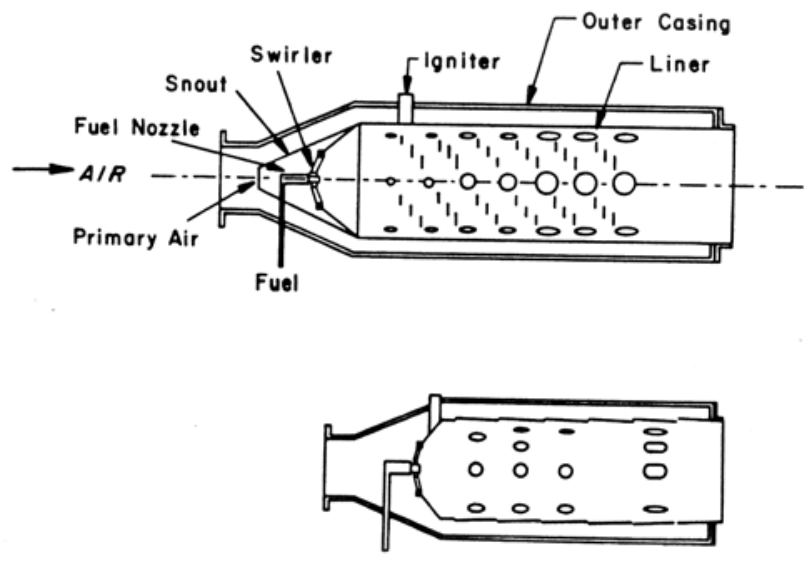

Figure 2.25 Combustor models used by Goldstein, Lau, and Leung (1983). Above, the first combustor with the entry holes for the secondary air arranged axisymmetrically. Below, the General Electric Company combustor with secondary holes arranged non-symmetrically.
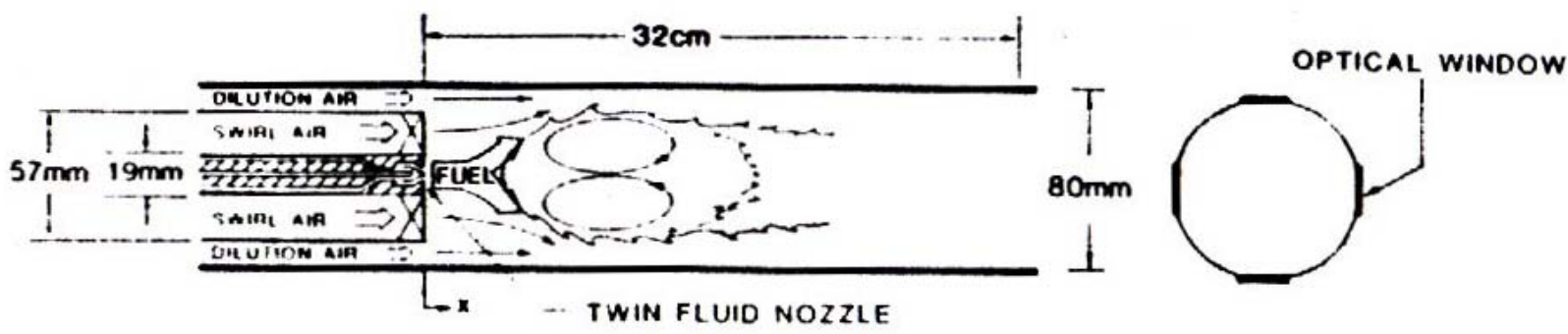

1(a) The axisymmetric can combustor (ASCC)

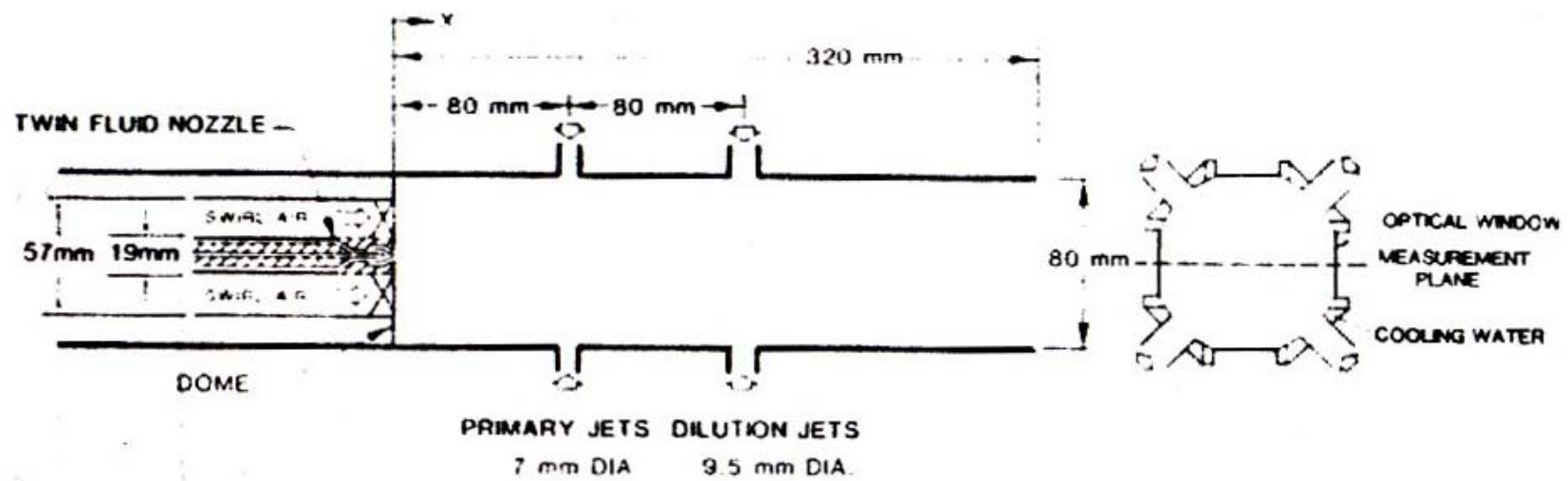

1(b) The wall jet can combustor (WJCC)

Figure 2.26 Schematic of the combustor model used by Cameron, Brouwer, Wood, and Samuelson (1989). 

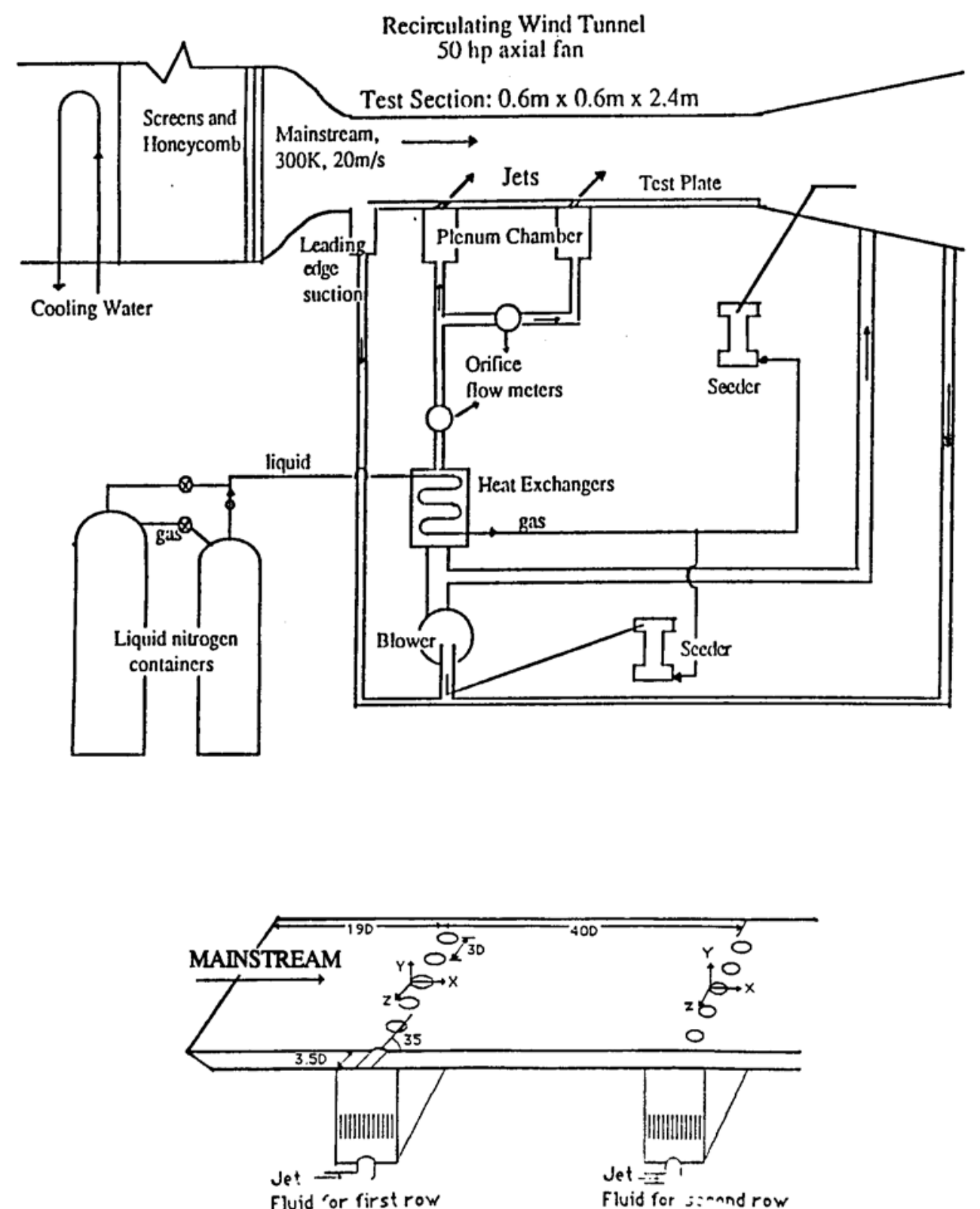

Figure 2.27 Schematic of the test facility (above) used by Sinha, Bogard, and Crawford (1990). Below, an up-close graphic of the film-cooling test section. 


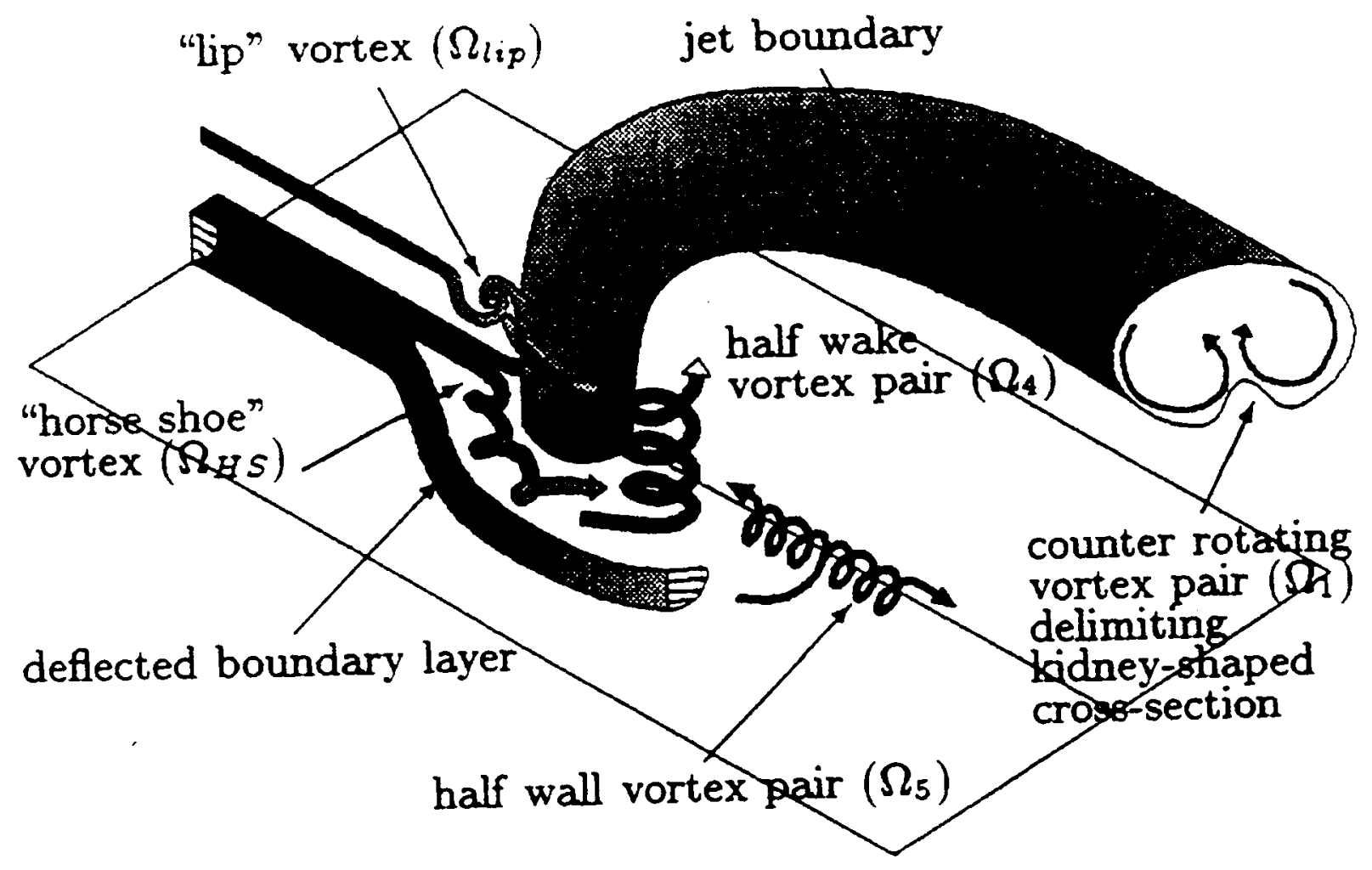

Figure 2.28 Schematic of the jet in crossflow model developed by Sgarzi and Leboeuf (1997). 


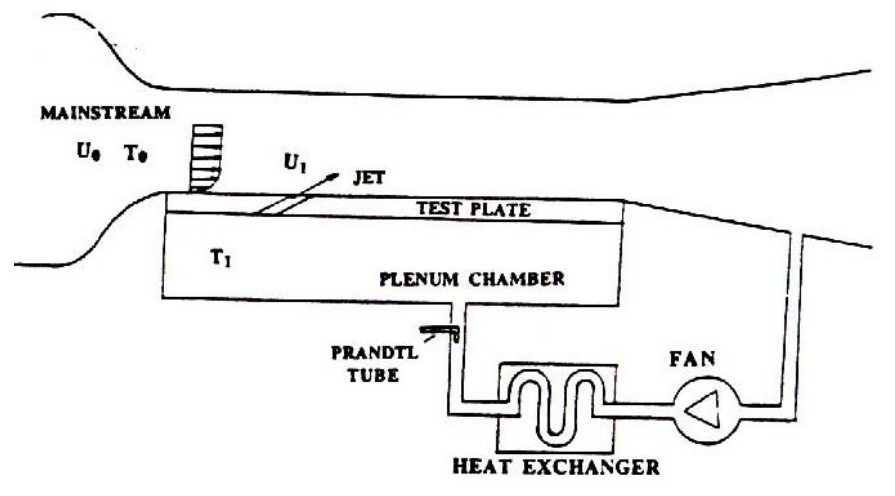

A schematic illustration of the experimental setup

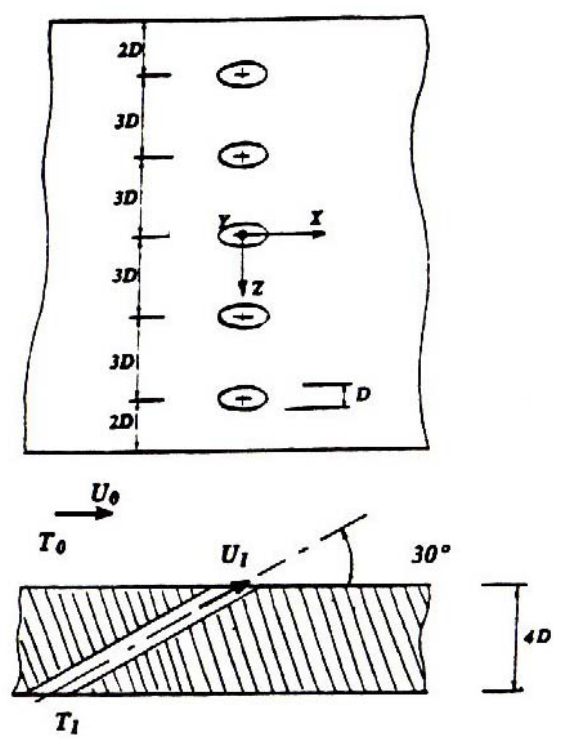

The geometry and coordinate system used

Figure 2.29 Schematic of Rydholm (1998) test rig. 


\section{Chapter 3: Experimental Facilities and Instrumentation}

To meet the goals of this study, which were to understand the complex thermal and velocity fields within a combustor, one must first become familiar with the facility in which the experiments were conducted. A key to acquiring accurate and representative data was having good measurement resolution along with the proper measurement instruments and techniques. This was accomplished through the use of a large-scale combustor simulator housed within a wind tunnel facility, and various measurement instruments including pressure transducers, thermocouple probes, and a laser Doppler velocimeter (LDV). The first section of this chapter gives an overview of the wind tunnel facility including the following: a detailed description of the combustor simulator, the thermal system, and finally typical operating conditions. The second part of this chapter describes in detail the various measurement instruments and techniques used in this research, while the last section finishes with a complete uncertainty analysis.

\subsection{Wind Tunnel Facility}

All of the experimental results presented in this study were conducted in a largescale, low-speed, closed-loop wind tunnel facility belonging to the Virginia Tech Experimental, Computational, and Convection Lab (VTExCCL) located in the basement of Randolph Hall. The purpose of this wind tunnel, shown in Figure 3.1, was to model the flow through a combustor and the first stage of turbine vanes in an actual aircraft gas turbine engine. The flow was driven by a $50 \mathrm{hp}, 0-60 \mathrm{~Hz}$ Joy Technologies axial fan. Precise control of the fan's speed, i.e. the mass flow through the closed loop, was achieved through the use of a Toshiba variable frequency inverter. Directly downstream of the fan, the air flowed through a primary finned-tube heat exchanger, which cooled the recirculating air. The flow was then split into a primary path and a secondary path, which was essential to modeling of the gas turbine engine. The primary path simulated the hot gasses flowing through the combustor, while the secondary path provided the necessary flow to simulate combustor liner cooling and dilution. 
Downstream the flow split in the primary flow path, a perforated-aluminum plate with $7.2 \%$ open-area (Figure 3.2 ) was used to provide the necessary pressure drop to direct enough flow into the secondary flow path. The perforated plate was fitted with tubes, $3.5 \mathrm{~cm}$ in diameter and $5.1 \mathrm{~cm}$ in length, which were inserted into each of its 90 holes. The tubes were constructed out of thin sheet metal and aided in straightening the flow as it came around the wind tunnel's $90^{\circ}$ bend. These tubes resulted in a uniform flow field hitting the downstream heaters, which was critical in obtaining a uniform temperature distribution in the vertical and horizontal directions as the mainstream flow entered the combustor simulator.

The heating element in the primary path was a bank of Watlow finned stripheaters, which provided the required heat to simulate the hot mainstream within the combustor simulator. Following the heater elements, the primary path flow was directed through a flow-conditioning section consisting of screens and honeycomb. This conditioning section straightened and damped out the overall turbulence level of the flow.

The secondary flow paths, shown in Figure 3.1 as the blue section, each contained a secondary finned-tube heat exchanger. This secondary heat exchanger provided the required temperature drop in the air for injection through the combustor simulator's filmcooled liner panels and dilutions jets. The secondary flow then passed through various plenums all of which had adjustable shutter mechanisms that controlled the flow rates through each of the combustor's liner panels and dilution jets. After being ejected through the liner panels and dilution jets, the flow mixed with the combustor simulator's hot primary flow and traveled through the turbine-vane cascade, completing the flowloop.

Following the combustor simulator was a linear turbine vane cascade. Although no experiments were conducted within the turbine vane cascade in this study, it is important to briefly note its main features to provide the proper background and motivation behind the combustor simulator design (Radomsky, 2000). The vane cascade constructed for this wind tunnel modeled a single sector (two pitches) of the first stage of turbine turning vanes inside of the Pratt and Whitney 6000 series aircraft engine. Furthermore, to provide exceptional measurement resolution, the model turbine vanes were scaled up by a factor of nine. While only one sector was modeled, periodicity 
between the two flow passages was maintained by bleeds on the corresponding suction and pressure side of the outer vanes (see Figure 3.3). Lastly, the Reynolds number at the turbine inlet was used to dictate the combustor exit conditions.

\section{Combustor Simulator}

Of the entire wind tunnel facility, the combustor simulator was the most integral and important segment for my study. It was the flow and thermal fields within this section that were the focus of this study; therefore, a detailed description is necessary and appropriate. The design and benchmarking of the combustor simulator were described in detail in a paper by Barringer et al (2001) and in the thesis presented by Barringer (2001); however, a number of the relevant details are repeated in this chapter for completeness.

The combustor simulator was designed for an existing linear vane cascade, and maintained the same general shape of a gas turbine aero-engine. Modeled symmetrically about the mid-span, the combustor simulator began with a region of constant crosssectional area and then underwent a contraction in the spanwise direction before mating up with the turbine section. Since the vane cascade was scaled up by a factor of nine, all of the combustor simulator features were also scaled by the same amount. Again, the motivation behind the larger scaling was to provide fine measurement resolution within the turbine vane cascade, which also translated directly to combustor simulator. Moreover, since the turbine vane cascade was constructed with flat endwalls, the combustor simulator was also constructed with flat liner panels as opposed to the true annular geometry of an actual combustor.

The two parameters that determined the overall design of VTExCCL's combustor simulator were the turbine inlet Reynolds number and a non-dimensional acceleration parameter, $K$. In the first step of the design, a turbine inlet Reynolds number, provided by Pratt and Whitney, of $\mathrm{Re}_{\text {in }}=2.2 \times 10^{5}$ was matched. The exit Reynolds number along with the inlet area of the turbine (the exit area of the combustor) dictated the massaveraged exit velocity of the combustor. The exit area of the combustor equaled the inlet area of the turbine section, which was set by maintaining the proper geometric scaling of the three-vane, two-passage, linear cascade. 
Pratt and Whitney provided a Mach number distribution along several axial locations of the combustor. By using this distribution along with a mass-averaged temperature distribution, the acceleration of the flow in the combustor was determined. The acceleration parameter is defined as follows:

$$
K=\frac{v}{u_{\infty}^{2}} \frac{d u_{\infty}}{d x}
$$

where $v$ is the kinetic viscosity, $u_{\infty}$ is the free stream velocity, and $\frac{d u_{\infty}}{d x}$ is the free stream velocity gradient in the axial direction. Knowing the exit velocity, the specified mass flow distributions through the liner-panels and dilution, and the overall axial length of the contraction, the inlet area to the contraction was determined by matching the acceleration parameter.

The final combustor simulator design resulted in an inlet of $111.8 \mathrm{~cm}$ (1.2 sectors) in width and $99.1 \mathrm{~cm}$ in height. The additional width allowed for the sidewall boundary layers to be removed from the center of the two vane passages. The overall length of the combustor was $156.9 \mathrm{~cm}$, while the contraction angle was 15.8 degrees. At the inlet, the cross-sectional area (based on the entire combustor width) was $1.11 \mathrm{~m}^{2}$, while at the exit the cross-sectional area had been reduced to $0.62 \mathrm{~m}^{2}$, giving an area contraction of 1.8. Furthermore, since $55 \%$ of the exit flow was added through the liner and dilution flow, the accelerated inviscid velocity at the combustor exit was 4 times greater than at the simulator inlet.

A global coordinate system was chosen, and all coordinates $(X, Y$, and $Z)$ were non-dimensionalized by the combustor length $(L)$, sector width $(W)$, and inlet height $\left(H_{i n}\right)$, respectively (refer to Figure 3.3). The contraction began at a $X / L$ location of 0.51 , which was roughly at the streamwise center of the combustor.

The combustor simulator consisted of a streamwise series of four film-cooled panels, which were symmetric on the top and bottom of the combustor simulator. These panels began approximately 2.7 vane chords $(\sim 1.6 \mathrm{~m})$ upstream of the turbine test section. The first two panel lengths were 39 and $41 \mathrm{~cm}$ while the third and fourth panels 
were $37 \mathrm{~cm}$ and $43 \mathrm{~cm}$. In addition, the first two panels were flat to maintain a constant cross-sectional area, while the last two panels were inclined at $15.8^{\circ}$ to give the desired contraction area at the combustor exit. The panels were constructed of $1.27 \mathrm{~cm}$ thick urethane foam, whose low thermal conductivity $(k=0.037 \mathrm{~W} / \mathrm{mK})$ allowed for adiabatic surface temperature measurements. The film-cooling hole pattern, which is shown in Figure 3.4, was configured in equilateral triangles that were spaced evenly across the panel surface. A water jet was used to cut the staggered rows of film-cooling into the urethane foam. The film-cooling holes were $0.76 \mathrm{~cm}$ in diameter and drilled at an angle of 30 degrees from the horizontal surface. Furthermore, the length of each film-cooling hole was $2.5 \mathrm{~cm}$, which resulted in a hole length to diameter ratio $(L / D)$ of 3.3 . Within an actual combustor, the purpose of the film-cooling jets is to provide a cool layer of air that protects the material of the combustor's inner and outer casing. Without this filmcooling, the material would surely begin to melt, eventually leading to catastrophic consequences.

Within the second and third panels are two rows of dilution holes that have been included by our design (see Figure 3.3). The dilution holes within the second panel (first row) were directed vertically, while the dilution holes in the third panel (second row) were angled at $15.8^{\circ}$ from the vertical axis. The diameters of the dilution holes were designed to insure that the dilution-to-mainstream momentum flux ratios, as well as the percent mass-flow addition from the dilution, matched that of an actual aero-engine. The first row of dilution holes had three evenly spaced holes with the center hole in alignment with the center of the simulator and the vane stagnation. This first row was located 0.67 $\mathrm{m}$ downstream of the beginning of the combustor liner panels $(X / L=0.43)$. The diameter of the holes in the first row of dilution was $8.5 \mathrm{~cm}$.

The second row of dilution holes was located $0.90 \mathrm{~m}$ downstream of the start of the combustor simulator $(X / L=0.57)$. The diameter for the second row dilution holes was approximately 1.4 times that of the first, at $12.1 \mathrm{~cm}$, and their centerlines were staggered with respect to those of the first row. The supply chamber for the dilution flow was required to be some distance from the exit of the hole, which resulted in an $L / D=1.5$ for both rows. Since the combustor simulator was symmetric about the mid-span, both 
rows of dilution holes were aligned with one another in the pitchwise and streamwise directions.

The dilution flow injected in the combustor's mainstream serves to properly mix the fuel and air, thus insuring reasonably uniform combustion. Many combustors are often designed with a swirler that is then followed by dilution holes. The goal is that the dilution will produce the opposite sense of rotation as the swirler and thereby reduce the overall exiting swirl. Since a swirler was not simulated in my study, the dilution holes were aligned with one another with the intentions of having only a small exit swirl. Lastly, the dilution flow helps in lowering the overall temperature of the hot gasses exiting the combustor, so that thermal limits of the first stage turbine vanes are not exceeded.

Twelve plenums supplied the flow to the combustor simulator's eight liner panels and four sets of dilution rows (four liner panels and two dilution rows on the top and bottom each). Each entity had its own plenum supply chamber, which was fitted with a shutter flow control mechanism (see Figure 3.5). As mentioned before, the flow to these plenums came through the tunnel's secondary flow path, while the necessary amount of total mass flow in the secondary path was provided by a pressure drop created by the perforated plate downstream of the flow split.

All mass flow rates within the film-cooled liner panels of the combustor simulator were measured via pressure differences between the plenum total pressure and the freestream static pressure at each panel. A pressure tap was installed in every plenum, while each liner panel had a pressure tap placed flush to its surface. The pressure taps were placed at the beginning of liner panels one and two, while they were placed in the middle of panels three and four to properly account for the acceleration effects, due to the contraction. On the other hand, since the size of the dilution holes was relatively large in comparison to the size of its plenum size, space constraints did not allow for the construction of a plenum large enough to permit the flow to stagnate. Thus, the velocity through the dilution holes was set through pitot-tubes fixed at the exits of the outer dilution holes. Although, the combustor simulator was originally designed according to the mass flow rates provided by Pratt and Whitney, the system was extremely flexible. 
By simply replacing the perforated plate with a more open or closed one and adjusting the shutters, numerous combustor flow conditions could be simulated.

Lastly, the combustor simulator was fitted with removable Plexiglas panels on the inside of the tunnel and either a Lexan wall or two piece MDF wood and float glass wall on the outside. The removable Plexiglas panels were evenly spaced and had vertical access ports between them. These access ports were either plugged with Plexiglas strips when not in use, or could be fitted with brush seals to allow for temperature and pressure probes. Furthermore, the flexibility in removing these panels allowed for easy access inside the combustor for any necessary repairs and/or modifications. The large seamless Lexan window on the outside was originally intended for LDV measurements. After trail and error, however, it was determined that the bowing of the relatively large Lexan wall was not ideal for LDV measurements (see LDV subsection within this chapter). It is for this reason that the outside window was replaced with the two piece MDF wood and float glass wall. The MDF section of the outer wall extended about $9.8 \mathrm{~cm}$ downstream of the leading edge of the first combustor liner panel. The rest of the combustor was fitted with a custom cut float glass piece that allowed optical access throughout the simulator. It was due to the size and weight of the float glass that the entire outer wall of the simulator was not fitted completely fitted with glass. The glass provided a uniform, scratch-free surface that proved to be superior to Lexan when making optical measurements.

\section{Wind Tunnel Thermal Conditioning System}

As stated before, the VTExCCL wind tunnel was a large scale, low-speed design that simulated the flows within the combustor and the first stage of turbine vanes. By no means were the actual temperatures found within an actual engine replicated. This was a limitation resulting from a number of factors such as size, equipment, power, and melting points of the tunnel materials. However, to provide the most representative data with good measurement resolution, a reasonably large temperature gradient between the hot mainstream flow and the cold secondary flow was desired.

As mentioned earlier, downstream of the perforated plate and upstream of the flow conditioning elements, the mainstream flow passed through a heater to simulate the hot gasses of an actual combustor. This heater consisted of eighteen finned Watlow 
heating elements, $75 \Omega$ each, layered horizontally and perpendicular to the flow direction. The heating elements were grouped into three separate zones, each consisting of six individual heating elements. A Watlow Series 988 Controller controlled each zone separately and allowed one to specify the percentage of total available power to send to each zone, thus permitting a constant temperature across the span to be maintained.

The power source used for these heaters was an industrial 3-phase 480-volt line. This allowed for each of the zones to be wired in either a 3-Phase Wye or a 3-Phase Delta configuration. Initially in this study, the heaters were wired incorrectly and used the 3Phase Wye configuration (see Figure 3.6a). In each leg of the Wye configuration, two strip heaters were wired in parallel, giving an effective leg resistance of $37.5 \mathrm{ohms}$. Equation 3.2 gives the maximum power available from a single heater zone:

$$
P_{W y e}=\frac{V_{L}^{2}}{R}=\frac{480^{2}}{37.5}=6,144 \text { Watts }
$$

where $V_{L}$ is the line voltage of 480 volts and $R$ is the resistance of a single leg. The Wye configuration resulted in 6,144 Watts per zone and a total of 18,432 Watts when all three zones ran at full power $(100 \%)$.

When this incorrect wiring scheme was discovered, the system was re-wired using the 3-Phase Delta configuration. The Delta configuration allowed for a significantly larger amount of heat to be added to the mainstream flow. In the Delta configuration (Figure 3.6b), each leg still contained two strip heaters wired in parallel with an effective leg resistance of 37.5 ohms. However, from the Equation 3.3, it is clear that the Delta configuration results in three times as much power per zone:

$$
P_{\text {Delta }}=\frac{3 V_{L}^{2}}{R}=\frac{3 \cdot 480^{2}}{37.5}=18,432 \text { Watts }
$$

The Delta configuration resulted in 18,432 Watts per zone, and a total of 55,296 Watts when all three zones were at full power. 
The purpose of the main heat exchanger was to initially cool the mixed-out return flow from the combustor, while the secondary heat exchangers provided the necessary cooling to simulate the liner film-cooling and dilution flows. Earlier, it was shown that of the wind tunnel's three heat exchangers, one was located immediately downstream of the fan, whereas the other two where in each leg of the secondary channels. These heat exchangers were capable of running with either tap (building) or chilled water. Initially, however, there was only building water available. Unfortunately, the temperature of this water supply was subject to the weather and varied from $\sim 24{ }^{\circ} \mathrm{C}$ in the summer to $\sim 18{ }^{\circ} \mathrm{C}$ in the winter (Colban, 2001). As a result of these variations and the desire for a cooler secondary, a $40.4 \mathrm{~kW}$ chiller manufactured by Freeze Co. System was later installed. This chiller was capable of providing water as cold as $7.2{ }^{\circ} \mathrm{C}$. However, if the heat load on the heat exchangers was not large enough, the chiller would quickly cool the water to its maximum capability. To prevent the lines from freezing up, the chiller was programmed with an internal limit of $7.2{ }^{\circ} \mathrm{C}$ for the outgoing water supply. When this limit was reached, the compressor would stop operation until the outgoing water supply heated up by a couple degrees, at which point the compressor would then resume operation. This cyclic operating characteristic created temperature fluctuations in the secondary, and made it impossible to attain a steady-state operating condition. To bypass this behavior, the heaters were operated at their maximum capability. As a result of the significantly increased heat load on the heat exchangers, the compressor operated constantly and the coldest outgoing water temperatures attainable were nominally $12{ }^{\circ} \mathrm{C}$.

Typical flow rates through the primary flow heat exchanger and the secondary flow heat exchangers were roughly 70.7 and 26.5 liters/min (20 and 7 GPM), respectively. With water from the chiller passing through all of the heat exchangers and a full heat load provided by the heaters, a maximum temperature difference of $38{ }^{\circ} \mathrm{C}$ between the mainstream and coolant air was attainable. This difference was a reasonable increase over the $24{ }^{\circ} \mathrm{C}$ attainable with the tap water and heaters running in the Wye configuration.

Table 3.1 gives a complete description of the typical wind tunnel operating conditions. Table 3.2 describes the plenum and liner-panel operating conditions including the following: density ratio $(D R)$, momentum flux ratio $(I)$, discharge 
coefficient $\left(C_{d}\right)$, total-to-static pressure differences $(\Delta P)$, velocity ratio $(V R)$, mass flux ratio $(M)$, and the percent of the total combustor exit mass flow discharged by each panel and dilution row.

\subsection{Experimental Instrumentation and Measurement Techniques}

A number of measurement devices and techniques were used in this study. The following subsections focus on describing these devices and techniques in detail. The first section discusses the pressure system used to set the proper flows within the combustor simulator. Thermocouples and rakes used to make temperature measurements are described in the second section. The third section focuses on the data acquisition system and programs used to gather and process the temperature and pressure data. Lastly, the LDV system is described in detail, including the orientation for various measurement planes, and the rotational and non-orthogonal velocity transformations.

\section{Pressure Measurement System}

All of the necessary mass flow rates through the combustor simulator liners were set using measured total-to-static pressure differences, while pitot-tube total-to-static pressure measurements were used to set the dilution jet velocities. These pressure differences were read using any of the eight different linear pressure transducers, manufactured by Setra or Omega, which were available in the following ranges: 0-62 Pa, 0-125 Pa, 0-498 Pa, 0-623 Pa, 0-1245 Pa, and 0-2491 Pa (0.25" $\mathrm{H}_{2} \mathrm{O}, 0.50 " \mathrm{H}_{2} \mathrm{O}, 2.0$ " $\mathrm{H}_{2} \mathrm{O}, 2.5^{\prime \prime} \mathrm{H}_{2} \mathrm{O}, 5.0$ " $\mathrm{H}_{2} \mathrm{O}$, and 10.0" $\mathrm{H}_{2} \mathrm{O}$,). For convenience, a box containing all of the pressure transducers was constructed as shown in Figure 3.7. This box housed and protected all of the transducers under a Plexiglas lid, on which all of the necessary transducer connections were mounted. The lid featured on/off switches, a set of high/low pressure inputs, and BNC connections for the output voltage of each of the transducers. The Setra and Omega transducers required 9-30 and 12-36 $\mathrm{V}_{\mathrm{DC}}$ of excitation, respectively. Power was supplied to all of the transducers though one input that required two 12-volt lantern batteries wired in series ( 24 volts across the power terminals). Since 
the transducers drew an extremely small amount of current, these two batteries were more than sufficient to run one or all of the transducers simultaneously for an extended period of time.

Although all of the transducers had span and zero adjustments that allowed for fine-tuning to the slope and offset calibration coefficients, these coefficients were determined and set by the manufacturer using a best-fit straight-line method. The nonlinearity errors of the transducers were within a maximum of $\pm 1 \%$ of their full-scale (i.e. the maximum pressure read by each transducer) while the majority was within $\pm 0.2 \%$ of their full-scales. Lastly, the zero pressure and full-scale output signals ranged from $0.05-$ $5 \mathrm{~V}_{\mathrm{DC}}$ with errors in these voltages no greater than $\pm 1 \%$ of full-scale. For the $0-2491 \mathrm{~Pa}$ pressure transducer, the output signals ranged from $0.05-10 \mathrm{~V}_{\mathrm{DC}}$ with errors no greater than $\pm 0.5 \%$ of full-scale See Appendix A for a complete listing of transducer models, serial numbers, coefficients, and non-linearity.

As already mentioned, each liner panel and its plenum were fitted with a static and total pressure tap, while the dilution jets had pitot-probes. All of the static and total pressure taps were dedicated to their own channel on a 24-channel, three-wafer scanivalve shown in Figure 3.8. In all, there were twelve differential pressures required including eight liner panels and four rows of dilution (top and bottom). It was the output from each of the dedicated wafers, one for all the static pressures and another for all of the total pressures, which was connected to the low and high inputs of the pressure transducer. This simple configuration allowed one to switch through each channel and set the desired mass flows quickly and efficiently.

\section{Thermal Field Measurements}

Thermocouples were utilized to monitor temperatures in the secondary, the inlet temperatures in the mainstream, and in taking various temperature fields within the combustor. All of the temperature measurements in this study were made using 30-gage, type E thermocouples that were connected to the data acquisition box using 20-gage thermocouple extension wire. The thermocouple beads were made using a Tigtech argon-gas thermocouple welder, which insured that no third metal was introduced. The introduction of a possible third metal could result in erroneous readings for it introduces 
another junction that was not accounted for in calibration. Furthermore, by using a thermocouple welder, uniform and extremely small spherical beads were formed resulting in a smaller thermal mass and minimal response time. The spherical beads ranged in diameter from a minimum of $0.8 \mathrm{~mm}$ to a maximum of $1 \mathrm{~mm}$.

Two thermocouple rakes were used to quantify the thermal fields, however, the majority of the temperature fields were taken using a 21-probe thermocouple rake. The rake spanned a total distance of 10.2 centimeters with thermocouples evenly spaced every $5.1 \mathrm{~mm}$. Each thermocouple probe on the rake consisted of a $5.1 \mathrm{~cm} \mathrm{long,} 2.5 \mathrm{~mm}$ outer diameter aluminum shaft that provided rigidity and allowed for the thermocouple wire to be encapsulated. The approximate flow blockage through the rake's cross-sectional area (between each probe and not including the base) was 50\%; however, since the thermocouples were placed a reasonable distance from the rectangular wooden base of the rake (the largest flow obstruction), the disruption to the flow created by this blockage was considered to be minimal. Each thermocouple bead was fixed approximately 6.4 $\mathrm{mm}$ from the end of the aluminum shaft to minimize heat conduction effects from the aluminum rod to the thermocouple. Figure 3.9a shows the typical construction of the thermocouple rakes used in this study.

It is important to note that some of the later temperature fields were taken using a newly constructed rake. The new rake was very similar to the original 21-probe rake, with only minor changes. Like the older rake, the new rake spanned a total of 10.2 centimeters; however, this rake was fitted with only 17 thermocouples that were evenly spaced every $6.4 \mathrm{~mm}$. In addition, the new rake utilized $6 \mathrm{~cm}$ long, $1.6 \mathrm{~mm}$ outer diameter brass tubes that had its thermocouple beads placed approximately $3.2 \mathrm{~mm}$ from the end of each tube (see Figure 3.9b). Through experimentation and comparison with the older rake, it was determined that a $6.4 \mathrm{~mm}$ spacing between probes was sufficient to resolve the thermal gradients while reducing the flow blockage to 24 percent. Lastly, an airfoil shaped piece (as opposed to the rectangular wooden piece) was chosen for the base of this new rake to insure that it was as aerodynamically non-intrusive as possible. Another VTExCCL student, Andrew Lethander, performed measurements using a single probe and compared those to the temperatures read by the multiple probe rake. His findings indicated that both gave the same temperature profiles. In all, the improvements 
in the new rake over the old were primarily to make it as non-intrusive as possible. This proved to be important in very turbulent (i.e. large velocity fluctuations) regions, but made little to no difference in other regions of the tunnel. Furthermore, although there are benefits of the new rake over the old, the effects on the resultant temperature fields taken by each was minimal, as shown in the uncertainty analysis of this chapter.

\section{Data Acquisition System}

A data acquisition system manufactured by National Instruments was used in gathering all of the temperature and pressure data in this study. This system consisted of three SCXI-1303 terminal blocks, three SCXI-1100 modules, a SCXI-1000 chassis, and a model NI AT-MIO-16E-2 data acquisition card (DAQ).

The SCXI-1303 terminal blocks (Figure 3.10a) served as the immediate connection for all of the pressure transducers and thermocouples. Each block had 32 available input channels and of the three blocks, two were dedicated for thermocouples and one for pressure transducers. Each terminal block was directly plugged into their individual SCXI-1100 module (Figure 3.10b). The SCXI-1100 module's main purpose was to serve as a multiplexer and an amplifier. The SCXI-1100 switched between the 32 input voltages it received from its terminal block and then sent out all of the signals on a single line. Furthermore when detecting extremely small voltages, such as those read by thermocouples, the module had an adjustable gain that amplified the small thermocouple voltages before they were sent to the DAQ card in the computer. For the pressure measurements, the adjustable gain was set to unity while for the temperature measurements the gain was 2,000. The three modules were housed within the SCXI1000 chassis (Figure 3.10c). This rugged, low noise chassis' main function was to power the SCXI modules and handle all timing, triggering and signal routing between the DAQ card in the computer and the modules. Finally, the signals were sent to the NI AT-MIO16E-2 (Figure 3.10d) data acquisition card via a shielded cable. This card was capable of accepting as many as 16 single-ended analog inputs from multiple SCXI chasses. Its primary function was to read the analog signals received from the chassis, convert them to digital signals, and send them out for processing. The NI AT-MIO-16E-2 DAQ card 
was capable of processing 500 kilo-samples per second, while giving 12-bit resolution (National Instruments, 2002).

On the user end, the software interface used to acquire and process all of the data was National Instruments' LabView program. This program allowed the user to custom build his or her own visual interface (vi) to display and manipulate the data in any fashion he or she may choose. The vi's used in this study were relatively straightforward. For both the pressure and temperature vi's, the main inputs were the module address, number of channels to scan, total number of samples per point, and the sampling frequency. When setting the mass flow rates within the combustor, pressures were sampled at a rate of 1000 samples/second with a total of 4000 samples. This resulted in a pressure reading every four seconds, which was fairly quick in response while providing accurate results.

When taking temperature measurements, 10,000 samples at a rate of 1,000 samples per second were generally taken. This sampling time of ten seconds was adequate in non-turbulent regions, i.e. before the dilution flow. However, it was later determined that when taking data in or around dilution flow affected regions (turbulent regions), a slightly higher sampling time was required in order to average out the large fluctuations created by turbulent eddies, vortices, etc. In these turbulent regions, the number of total samples was changed to 5,000 and the sampling rate was dropped to 330 samples per second, resulting in a sample time of approximately 15 seconds; the overall sample size was reduced because there was no difference in temperatures between 10,000 and 5,000 samples (addressed in Section 3.3). Furthermore, it is worth mentioning that many of the planes were done using an older computer to which the DAQ system was connected. Due to limitations in the speed of the computer's processor, the processing times for a single temperature measurement were often on the order of $\sim 14-15$ seconds in non-turbulent regions and around $\sim 20$ seconds in turbulent regions. However, this problem has been remedied with the replacement of the older computer, and the processing times now remain true.

\section{Laser Doppler Velocimeter System}

Detailed flow field mapping was conducted using a three-component fiber optic laser Doppler velocimeter (LDV). This LDV, shown in figure 3.11, consisted of a five 
Watt Spectra Physics argon-ion coherent Laser, a TSI model 9201 Colorburst beam separator, TSI model 9832 two-component (green and blue lasers) and single-component (violet laser) fiber optic probes, a TSI model 9230 ColorLink Multicolor Receiver, and a TSI model IFA 755 Digital Burst Correlator. Shift magnitudes, signal filters, traverse control, data gathering, and time weighted bias corrections and matrix manipulations of gathered data were all conducted through TSI's FIND software.

The multi-mode laser beam was transmitted to the Colorburst where it was split into three colors of light: green $(514.5 \mathrm{~nm})$, blue $(488 \mathrm{~nm})$, and violet $(476.5 \mathrm{~nm})$. This light then traveled through the optical fibers from the Colorburst to the fiber-optic probe. The probe focused the light to form a measurement volume, as well as gathered scattered light from particle passing through this measurement volume. The ColorLink served two main purposes. First, it provided the necessary excitation voltage to the Bragg cell within the Colorburst to implement shifting. Second, it received the scattered light signals from the fiber optic probe and converted them to a voltage that was then sent to the IFA 755 digital burst correlator. The IFA 755 was a signal processor, whereby it extracted the Doppler frequency information by digitizing the voltages received from the ColorLink into a format for the FIND software to process and provide final statistical data analysis/display.

The seed used in all of these experiments was olive oil. The seeder was a design recommended by TSI and can be seen in Figure 3.12 (Radomsky, 2000). The olive oil seeder consisted of a pre-built spray paint tank. The lid of the tank was constructed out of aluminum and had inlet holes for the compressed air, and an outlet for the atomized olive oil. The compressed air was injected through six rods that were attached to the aluminum lid and placed into the vat of olive oil. At the end of the six rods were four 0.1 $\mathrm{cm}$ diameter holes through which the air was injected. Located $0.05 \mathrm{~cm}$ above the holes was a disk with the same four holes drilled through it. According to TSI's direction, it was critical that the holes in the disk lined-up with those in the tube for the system to work properly. When air was injected through this system, the olive oil was atomized and the seeded air flowed past a deflector plate before it exited the pre-built spray paint tank. The function of the deflector plate was to block any abnormally large particles form passing through the system. This seeding method created olive oil droplets that 
were nominally $1 \mu \mathrm{m}$ in diameter (TSI, 1999). The advantages of olive oil over other conventional seed mediums, such as aluminum dioxide, was that it produced extremely light particles resulting in smaller amounts of needed seed, it was non-toxic or harmful to one's health, it did not create large deposits, and simple soap and water were all that were required for clean-up.

Movement of the probe/probes throughout the measurement planes was done using a Velmex traverse system. The system consisted of a Velmex VP9000 motor controller and two Unislide traverses. One traverse was for the vertical movement and the other was for either the streamwise or cross-pitch movement. Both of the traverses had a $0.95 \mathrm{~cm}$ diameter screw with a pitch of 10 turns $/ \mathrm{cm}$, and the smallest increment of translation was $10 \mu \mathrm{m}$.

In taking the streamwise flow field, only a two-component LDV setup was needed due to the flow symmetry (Figure 3.13). The two-component set-up used a 350 $\mathrm{mm}$ focusing lens without a beam expander and had a measurement volume of $90 \mu \mathrm{m}$ in diameter and $1.3 \mathrm{~mm}$ in length. The plane was acquired with the probe perpendicular to the outer wall surface. This allowed for the direct measurement of the streamwise, $u$, velocity component. However, to take measurements near the surface of the liner panel, the probe had to be tilted approximately 7.3 degrees (see Figure 3.14). Thus, to achieve the accurate vertical, $w$, component of velocity, a transformation had to be used. The actual velocities are given by equations 3.4 ,

$$
\begin{aligned}
& u=u^{\prime} \\
& w=\cos \left(\theta_{\text {tilt }}\right) \cdot w^{\prime}
\end{aligned}
$$

where $u$ and $w$ are the actual velocity quantities, $u^{\prime}$ and $w^{\prime}$ are the non-tilt corrected quantities, and $\theta_{\text {tilt }}$ is the tilt angle of the probe (TSI, 2000). Note that for the twocomponent case $u^{\prime}$ and $w^{\prime}$ are equal to the measured values by the green and blue laser crossing, $u_{g}$ and $u_{b}$.

For the two cross-pitch planes, where three-component velocity measurements were made, a two-probe set-up was used. To allow the measurement volume of the probes to reach the mid-pitch of the combustor simulator, a 2.6 magnification beam 
expander along with a $750 \mathrm{~mm}$ focusing lens was used. With the use of the beam expander, a reduction in the laser's diameter occurred, which in turn reduced the overall measurement volume by approximately 57 percent. For the $750 \mathrm{~mm}$ focusing lens, the measurement volume was $73 \mu \mathrm{m}$ and $1.3 \mathrm{~mm}$ in diameter and length, respectively. Ideally, the TSI model 9832 two-component and single component probes would be arranged perpendicular to one another, thereby allowing the direct measurement of $u, v$, and $w$ velocities. However, due to the location of the test section measurement window and the availability of only one traverse system, this was not possible. All measurements had to be conducted through a non-orthogonal system set-up, which was then later transformed to the actual components $u, v$, and $w$ using the FIND software. Furthermore, as with the two-component measurements, for the measurement volume to reach the combustor surface, an applied tilt to both probes was required. The actual threecomponent system set-up can be seen in Figure 3.15. Both probes were turned $13.3^{\circ}$ degrees inwards, while the vertical tilt angle was set again to $7.3^{\circ}$. The green and violet laser beam pairs resided in the tilted horizontal plane while the blue laser beam pair was in the tilted vertical plane and perpendicular to the green beam pair. In this nonorthogonal scheme (refer to Figure 3.16), contributions to the streamwise and pitchwise velocities were coupled within the measured green and violet quantities. The transformation equations used to back out the actual $u, v$, and $w$ velocities are given by equations 3.5:

$$
\begin{aligned}
& u^{\prime}=\frac{u_{g} \sin \theta_{v}+u_{v} \sin \theta_{g}}{\sin \left(\theta_{g}+\theta_{v}\right)} \\
& v^{\prime}=\frac{-u_{g} \cos \theta_{v}+u_{v} \cos \theta_{g}}{\sin \left(\theta_{g}+\theta_{v}\right)} \\
& w^{\prime}=u_{b}
\end{aligned}
$$

where $u^{\prime}, v^{\prime}$ and $w^{\prime}$ are the non-tilt-corrected orthogonal velocities; $u_{g}, u_{b}$, and $u_{v}$ are the probe measured velocities; and $\theta_{g}$ and $\theta_{v}$ are the $13.3^{\circ}$ turn angles, off the $Y$-axis, applied to the probes (TSI, 2000). Having applied the non-orthogonal to orthogonal correction, the $u^{\prime}, v^{\prime}$ and $w^{\prime}$ quantities were then corrected for the tilt angle applied to the probes. It is 
important to note, that the measured quantities were no longer used in the tilt transformation; it was solely the orthogonal quantities that were now of interest. Figure 3.17 shows that the tilt had no effect on the streamwise velocity, $u$; however, transformations to $v$ and $w$ must be applied and are given by equations 3.6

$$
\begin{aligned}
& u=u^{\prime} \\
& v=v^{\prime} \cos \theta_{\text {tilt }}+w^{\prime} \sin \theta_{\text {tilt }} \\
& w=-v^{\prime} \sin \theta_{\text {tilt }}+w^{\prime} \cos \theta_{\text {tilt }}
\end{aligned}
$$

where $u, v$, and $w$ are the true velocities within the combustor simulator and $\theta_{\text {tilt }}$ is the tilt angle applied to the probes (TSI, 2000). Equations 3.4-3.6 were altered to fit the probe orientation and variables used for the lab coordinate system unique to this study. Appendix B gives a detailed description of the original non-orthogonal and tilt transformations given by TSI, as well as figures documenting their original coordinate system, probe orientation, and variables. Additional figures and descriptions show the adjustments made to TSI's transformations, thus allowing the calculation of $u, v$ and $w$.

To accurately characterize the flow fields within the turbulent regions in and around the dilution flow, it was essential that the proper sample sizes and times were observed. In all of the velocity planes presented in this study, generally a coincidence window of 1 second was used to achieve reasonable data rates. The nominal sampling time was $\sim 40$ seconds with 15,000 data points taken for each component. However, due to the turbulent nature of the measured regions and the longer $750 \mathrm{~mm}$ focusing lens, fast data rates were often difficult to achieve. Most measurement locations had an average sampling time of approximately 100 seconds for 15,000 points in each component. A number of times, the data rates dropped so radically that the overall sample size was reduced to as low as 10,000 points in each component, and the resulting sampling time was up to 250 seconds. Regardless, the sample size was not the most important factor in obtaining representative data; the difference between measured velocities resulting from 15,000 points or 10,000 points was minimal (refer to Section 3.3), as long as an adequate sampling time was used. 
Of the three flow fields, the two cross-pitch flow fields were measured through a glass window on the outside of the test section, while the streamwise flow field was taken using a Lexan window. It was determined that when shooting any of the shifted/unshifted laser pairs perpendicular to the Lexan surface, occasional drops in data rate were experienced do to some bowing and imperfections in the Lexan surface. These two factors adversely affected the beam crossing, causing the beams to not perfectly overlap one another. For the two cross-stream planes, the beam-expanders had to be put on the probes. When using a longer length focusing lens, the available measurement volume is inherently reduced, making the precision of the beam-crossing the most important factor for attaining good data rates. It turned out that the effect of a longer length focusing lens along with shooting through the Lexan at an angle was unfavorable. The uniformity, lack of scratches and other numerous imperfections gave glass superior optical qualities in comparison to the Lexan allowing for good data rates while not having to repeatedly steer the beams within the probe on a regular basis.

\subsection{Uncertainty Analysis}

An uncertainty analysis was performed on each of the major thermal and flow variables presented in this study. The partial derivative method, described by Moffat (1988) was used to complete the uncertainty analysis. The total uncertainty in any given variable was defined as the square root of the sum of the squares of the precision uncertainty and the bias uncertainty.

The precision uncertainties were based on a 95\% confidence interval ( 2 standard

deviations). For the temperature measurements, the precision uncertainty was determined for a single thermocouple as well as for the thermocouple rake. To determine the precision uncertainty of the thermocouples, five measurements were taken in a high and low turbulence region. The standard deviation, based on a $95 \%$ confidence interval, of the five measurements at each location determined the precision uncertainty. The average of the precision uncertainties in the high and low turbulence regions then gave the overall precision uncertainty for the thermocouple. The precision uncertainty values 
were taken from a study conducted earlier by another VTExCCL student, Andrew Lethander. Since the data acquisition system and thermocouples used in Lethander's study were the same as those used in this study, this was assumed to be valid. Lastly, the bias uncertainty for the thermocouple measurements was $0.2{ }^{\circ} \mathrm{C}$.

In determining the precision uncertainty for the LDV measurements, five data points were taken in a highly turbulent field with dominant velocities in the streamwise and spanwise directions, as well as in a turbulent field with only a dominant streamwise velocity. Again, the standard deviations of the mean velocities were determined and then averaged together to give the overall uncertainty. The precision uncertainty determined from the streamwise velocity measurements was used for the green and violet beam pairs, while the spanwise precision uncertainty was used for the blue beam pair. A total of 15,000 points over a period of 50 to 100 seconds were taken for these measurements as well as most of the flowfield measurements. The same methodology described above was used in determining the precision uncertainties in the root-mean-square values. The bias uncertainty for all of the LDV measurements was $1.0 \%$ of the mean measured quantity. Table 3.3 gives a summary of the uncertainty analysis results. Detailed equations and calculations can be found in Appendix C. 
Table 3.1 Typical Tunnel Operating Conditions for a Standard Run

\begin{tabular}{|c|c|}
\hline Atmospheric Pressure $(\mathbf{M P a})$ & 98.82 \\
\hline Freestream Temperature, $\boldsymbol{T}_{\boldsymbol{c}}\left({ }^{\mathbf{}}{ }^{\mathbf{C}}\right)$ & 59.04 \\
\hline Coolant Temperature, $\boldsymbol{T}_{\boldsymbol{c}}\left(\mathbf{(}^{\mathbf{0}} \mathbf{C}\right)$ & 22.55 \\
\hline Freestream Density, $\boldsymbol{\rho}_{\boldsymbol{c}}\left(\mathbf{k g} / \mathbf{m}^{\mathbf{3}}\right)$ & 1.00 \\
\hline Coolant Density, $\boldsymbol{\rho}_{\boldsymbol{c}}\left(\mathbf{k g} / \mathbf{m}^{\mathbf{3}}\right)$ & 1.12 \\
\hline Fan Speed $(\mathbf{H z})$ & 40.00 \\
\hline Inlet Velocity, $\boldsymbol{u}_{\boldsymbol{c}}(\mathbf{m} / \mathbf{s})$ & 1.62 \\
\hline
\end{tabular}

Table 3.2 Typical Liner-Panel and Plenum Operating Conditions for a Standard Run

\begin{tabular}{|l|c|c|c|c|c|c|c|}
\hline & $D R$ & $I$ & $C_{d}$ & $\Delta P(P a)$ & $V R$ & $M$ & $\begin{array}{c}\text { \% of Exit Mass Flow } \\
\text { (bottom panel or row) }\end{array}$ \\
\hline Panel 1 & 1.12 & 9 & 0.739 & 21.53 (Plenum) & 2.83 & 3.18 & 1.19 \\
\hline Panel 2 & 1.12 & 9 & 0.733 & 24.21 (Plenum) & 2.83 & 3.18 & 2.96 \\
\hline Dilution Row 1 & 1.12 & 128 & 0.829 & 185.28 (Pitot-Tube) & 10.67 & 11.99 & 8.73 \\
\hline Panel 3 & 1.12 & 9 & 0.733 & 54.11 (Plenum) & 2.83 & 3.18 & 3.82 \\
\hline Dilution Row 2 & 1.12 & 32 & 0.912 & 103.50 (Pitot-Tube) & 5.34 & 6.00 & 8.82 \\
\hline Panel 4 & 1.12 & 9 & 0.739 & 154.27 (Plenum) & 2.83 & 3.18 & 2.05 \\
\hline
\end{tabular}


Table 3.3 Summary of Uncertainty Analysis

\begin{tabular}{|c|c|c|c|c|c|c|c|}
\hline Variable & $\begin{array}{c}\text { LDV } \\
\text { Components }\end{array}$ & $\begin{array}{c}\text { High } \\
\text { Value }\end{array}$ & $\begin{array}{c} \pm \text { Total } \\
\text { Uncertainty }\end{array}$ & $\begin{array}{c}\text { Percent } \\
\text { Uncertainty }\end{array}$ & $\begin{array}{c}\text { Low } \\
\text { Value }\end{array}$ & $\begin{array}{c} \pm \text { Total } \\
\text { Uncertainty }\end{array}$ & $\begin{array}{c}\text { Percent } \\
\text { Uncertainty }\end{array}$ \\
\hline$\theta$ & - & 0.92 & 0.016 & - & 0.044 & 0.016 & - \\
\hline$u(\mathrm{~m} / \mathrm{s})$ & 2 & 9.97 & 0.16 & $1.6 \%$ & -2.87 & 0.13 & $4.5 \%$ \\
\hline$u / u_{\text {in }}$ & 2 & 5.97 & 0.46 & $7.8 \%$ & -1.72 & 0.15 & $8.8 \%$ \\
\hline$w(\mathrm{~m} / \mathrm{s})$ & 2 & 12.67 & 0.15 & $1.2 \%$ & -1.30 & 0.075 & $5.7 \%$ \\
\hline$w / u_{\text {in }}$ & 2 & 7.59 & 0.58 & $7.7 \%$ & -0.78 & 0.074 & $9.5 \%$ \\
\hline$u^{\prime}(\mathrm{m} / \mathrm{s})$ & 3 & 6.88 & 0.11 & $1.5 \%$ & -0.96 & 0.092 & $9.6 \%$ \\
\hline$u(\mathrm{~m} / \mathrm{s})$ & 3 & 6.88 & 0.11 & $1.5 \%$ & -0.96 & 0.092 & $9.6 \%$ \\
\hline$u / u_{\text {in }}$ & 3 & 4.12 & 0.32 & $7.7 \%$ & -0.57 & 0.070 & $12.3 \%$ \\
\hline$v^{\prime}(\mathrm{m} / \mathrm{s})$ & 3 & 2.95 & 0.43 & $14.5 \%$ & -4.51 & 0.46 & $10.2 \%$ \\
\hline$v(\mathrm{~m} / \mathrm{s})$ & 3 & 3.26 & 0.43 & $13.1 \%$ & -4.15 & 0.46 & $11.0 \%$ \\
\hline$v / u_{\text {in }}$ & 3 & 1.95 & 0.30 & $15.1 \%$ & -2.48 & 0.33 & $13.4 \%$ \\
\hline$w^{\prime}(\mathrm{m} / \mathrm{s})$ & 3 & 5.79 & 0.094 & $1.6 \%$ & -2.00 & 0.077 & $3.8 \%$ \\
\hline$w(\mathrm{~m} / \mathrm{s})$ & 3 & 5.80 & 0.11 & $1.9 \%$ & -1.81 & 0.096 & $5.3 \%$ \\
\hline$w / u_{\text {in }}$ & 3 & 3.47 & 0.11 & $7.8 \%$ & -1.09 & 0.10 & $9.3 \%$ \\
\hline$u_{r m s} / u_{\text {in }}$ & - & 1.91 & 0.16 & $8.4 \%$ & 0.11 & 0.070 & $61.7 \%$ \\
\hline$v_{r m s} / u_{\text {in }}$ & - & 3.02 & 0.24 & $7.9 \%$ & 0.18 & 0.071 & $38.8 \%$ \\
\hline$w_{r m s} / u_{\text {in }}$ & - & 2.44 & 0.20 & $8.1 \%$ & 0.13 & 0.072 & $55.3 \%$ \\
\hline
\end{tabular}




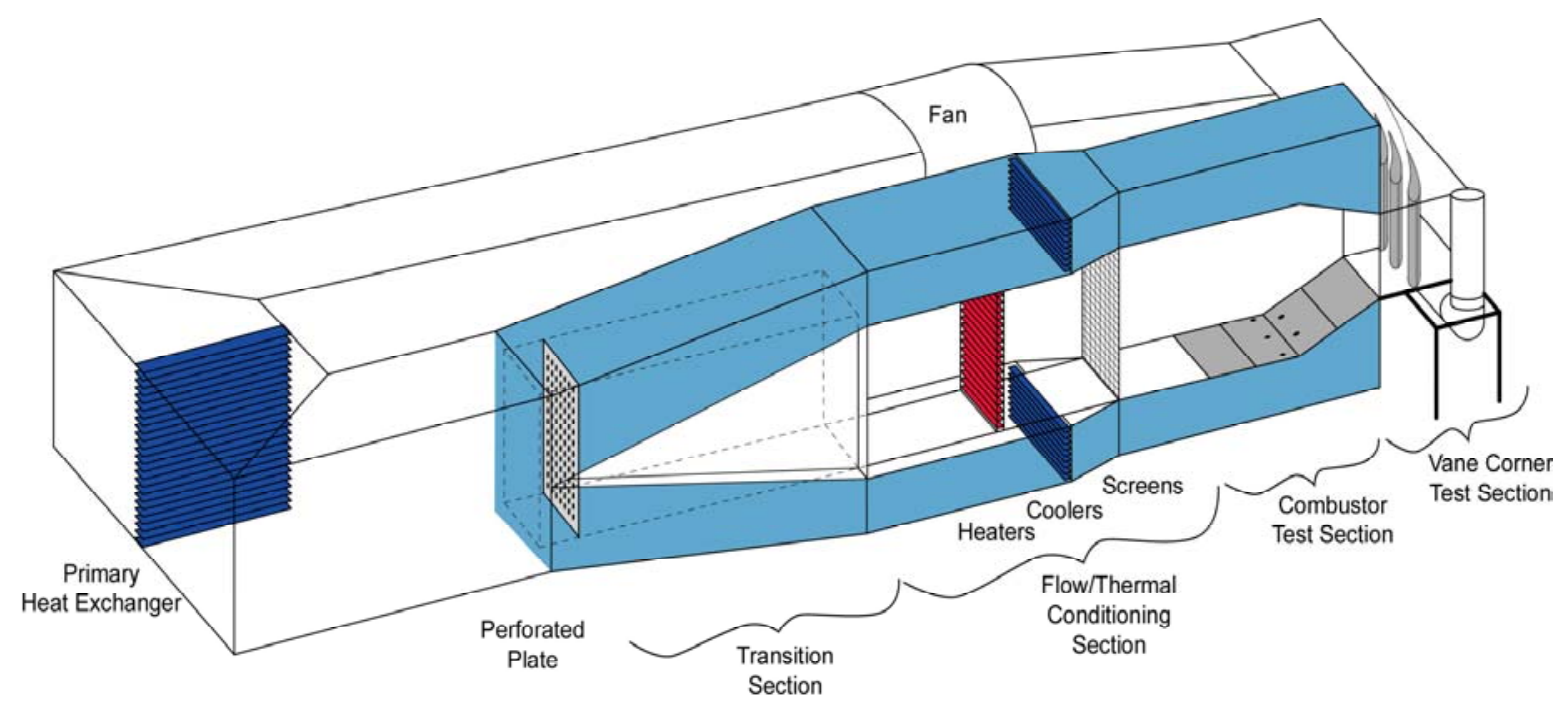

Figure 3.1 Overall schematic of the large-scale, low-speed, closed-loop wind tunnel facility.

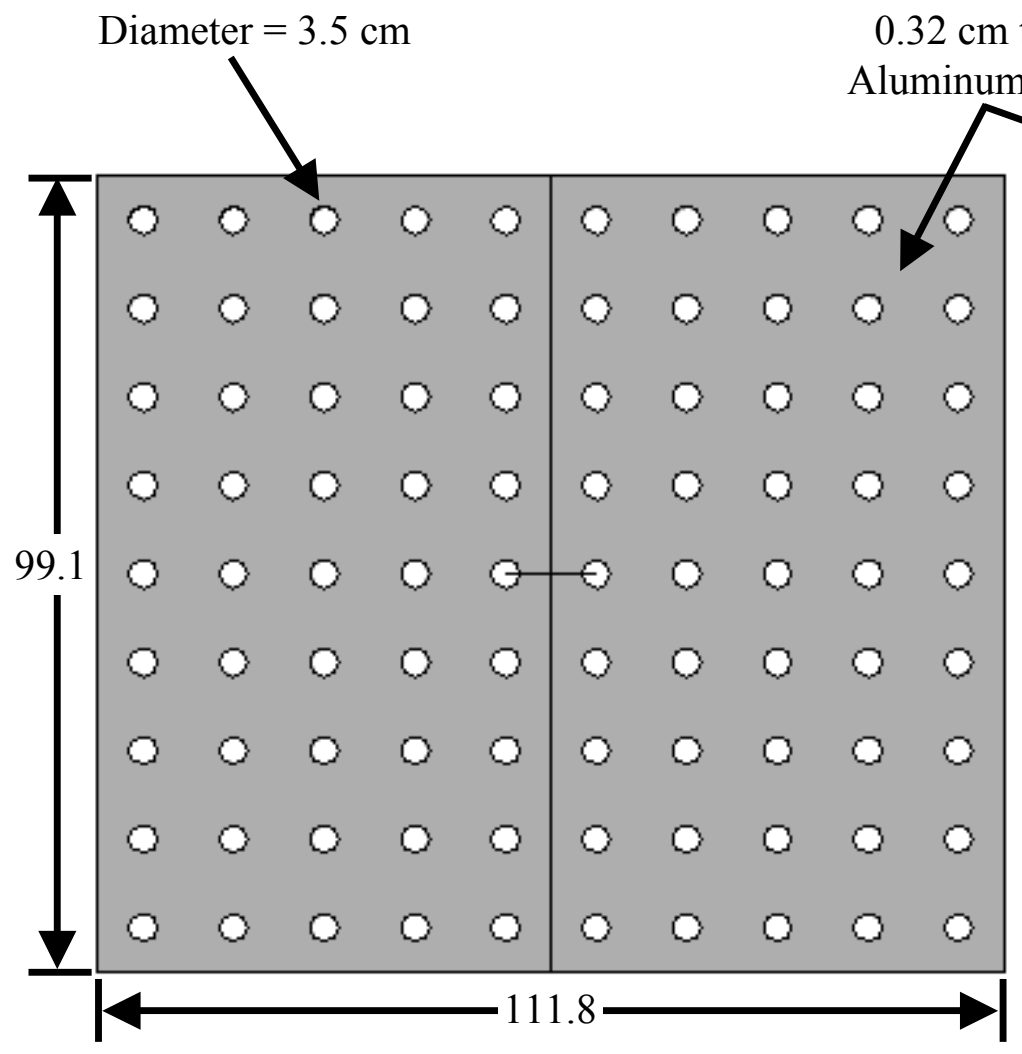

Front View thick

Aminum Sheet

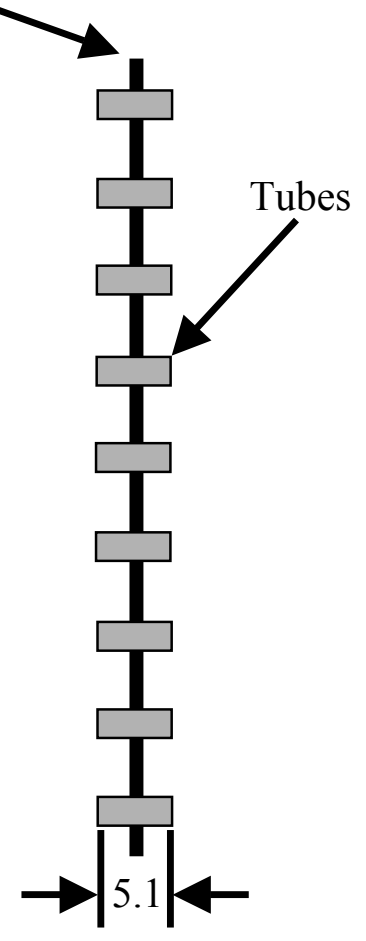

Side View

Figure 3.2 Perforated aluminum plate with $7.2 \%$ open area (dimensions are in centimeters). 


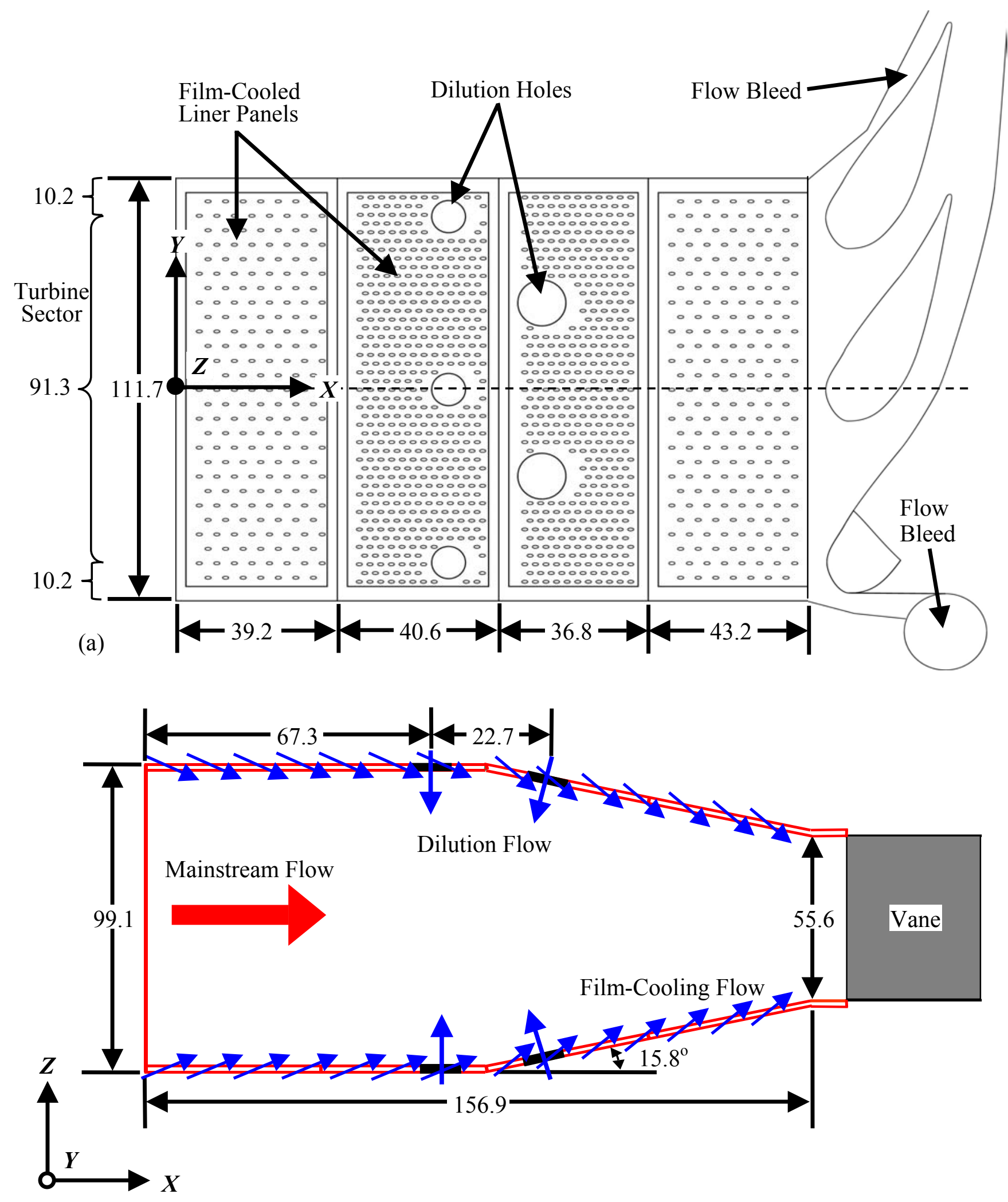

(b)

Figure 3.3 Schematics of the combustor simulator and turbine vane cascade showing (a) top view, and (b) side view (dimensions are in centimeters). 


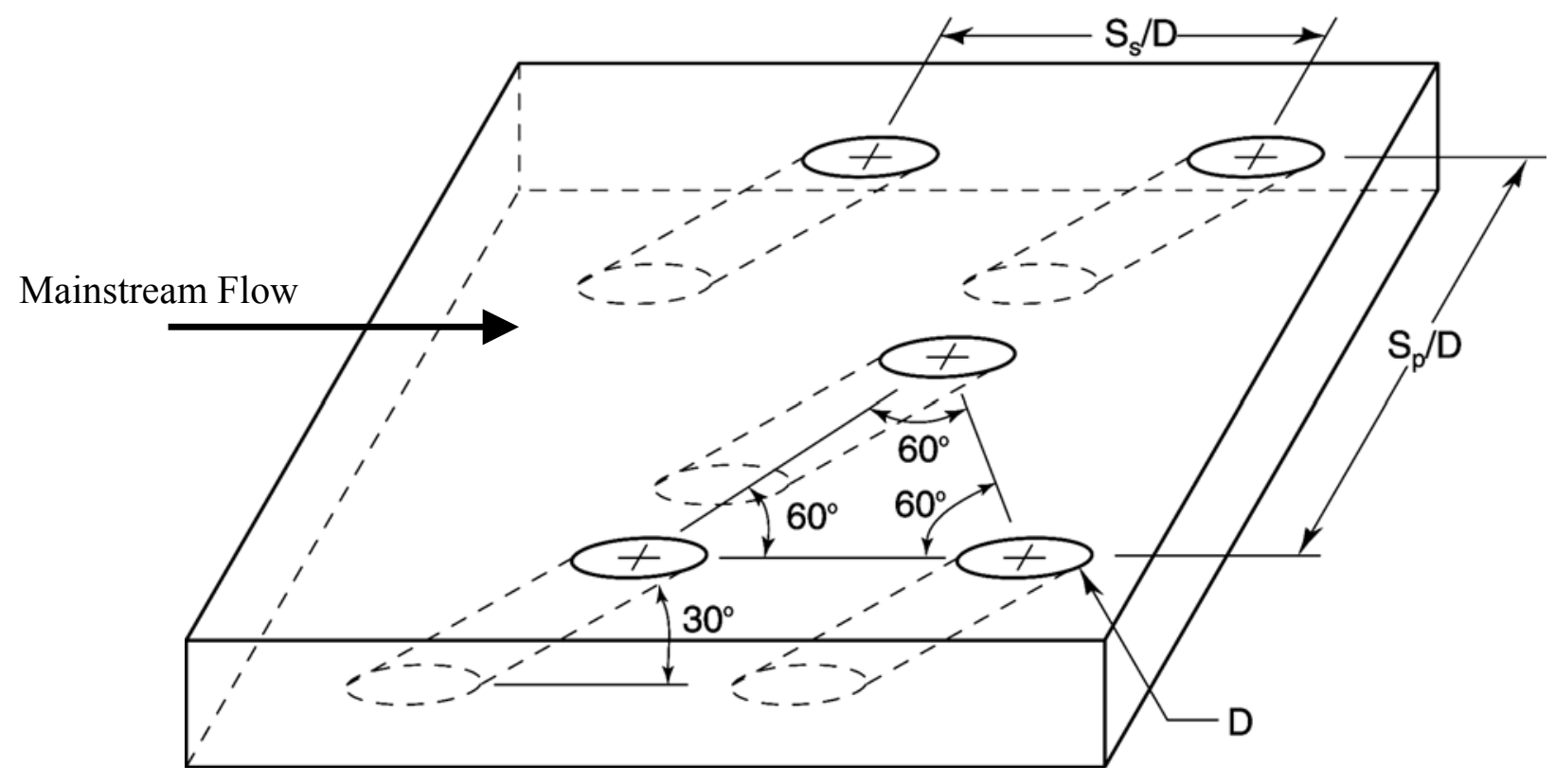

\begin{tabular}{|l|c|c|}
\hline & $\boldsymbol{S}_{\boldsymbol{p}} / \boldsymbol{D}$ & $\boldsymbol{S}_{\boldsymbol{s}} / \boldsymbol{D}$ \\
\hline Panel 1 & 10.1 & 5.8 \\
\hline Panel 2 & 6.1 & 3.5 \\
\hline Panel 3 & 6.1 & 3.5 \\
\hline Panel 4 & 10.1 & 5.8 \\
\hline Dilution 1 & $60\left(5.4 D_{l}\right)$ & $90\left(8.1 D_{l}\right)$ \\
\hline Dilution 2 & $60\left(3.8 D_{2}\right)$ & $121\left(7.6 D_{2}\right)$ \\
\hline
\end{tabular}

Figure 3.4 Schematics of an arbitrary liner panel showing the film-cooling hole pattern (Barringer, 2001). 

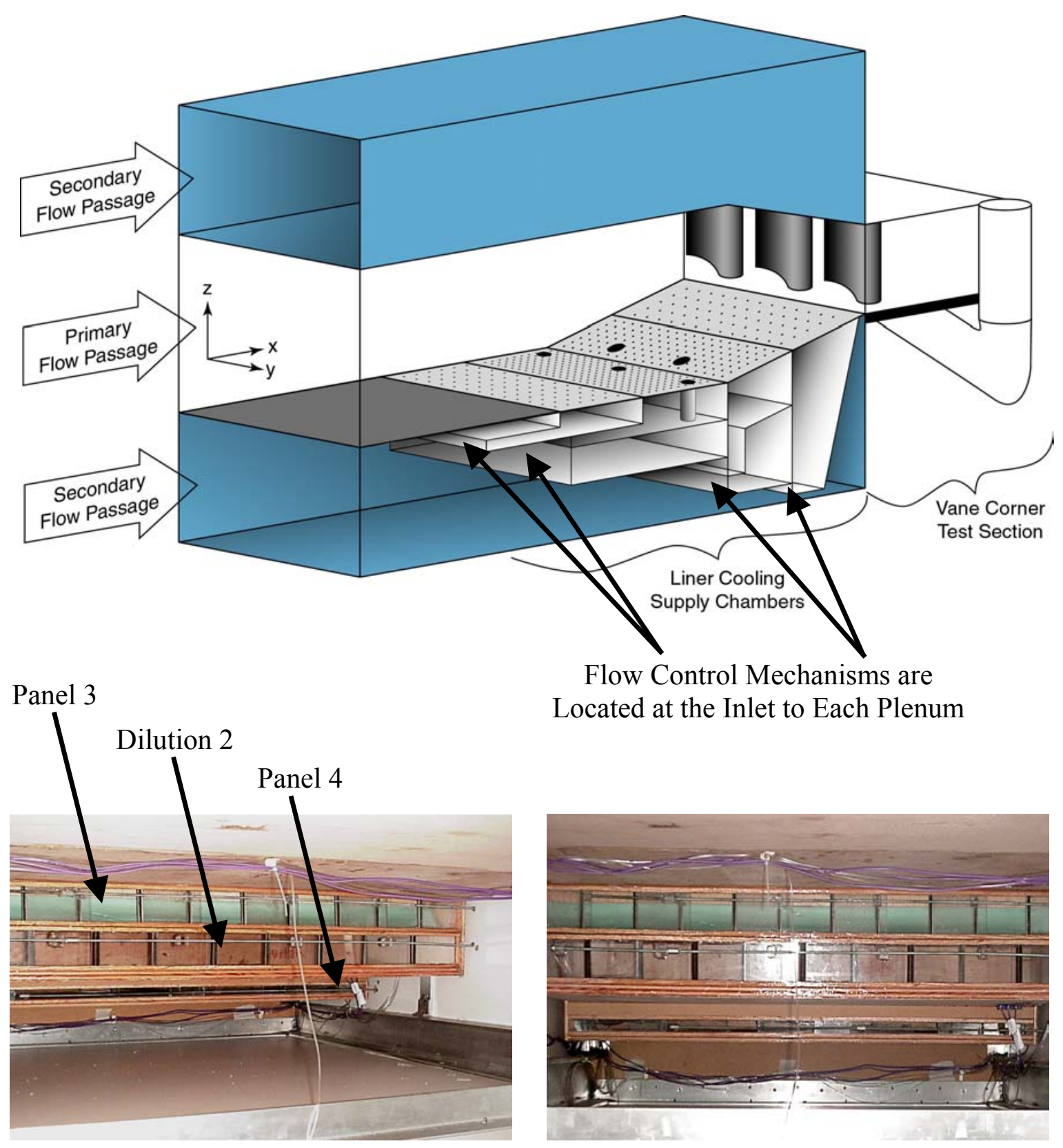

Figure 3.5 Cut-away of combustor simulator showing the plenum supply chambers, and the locations of the flow control mechanisms. Below are pictures of the flow control mechanisms for panels 3 and 4, as well as the second row of dilution. 

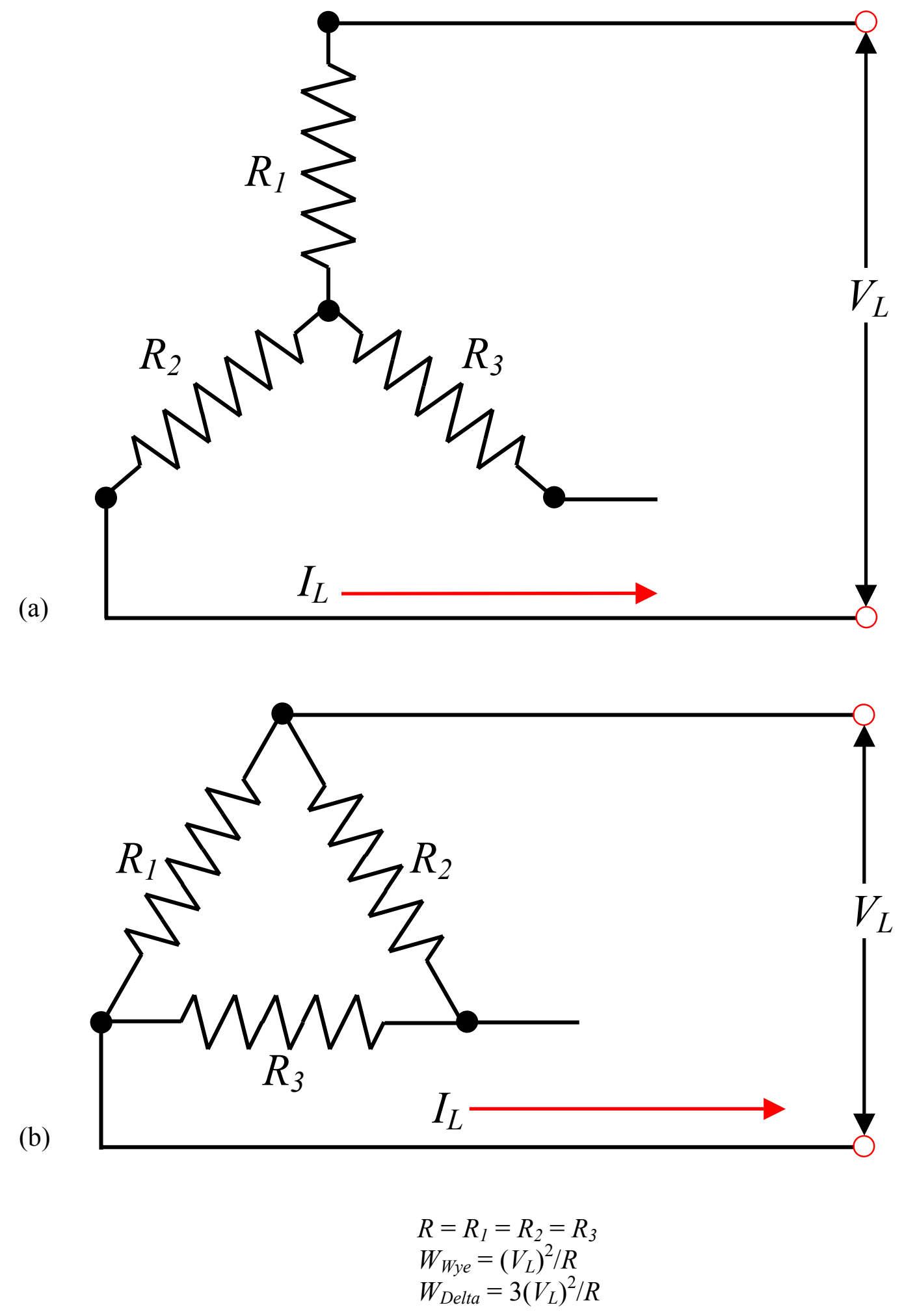

Figure 3.6 Wiring connections for a single heater bank in the (a) Wye and (b) Delta configuration $\left(R_{1}, R_{2}\right.$, and $R_{3}$ are the effective resistance of two strip heater elements in parallel). 


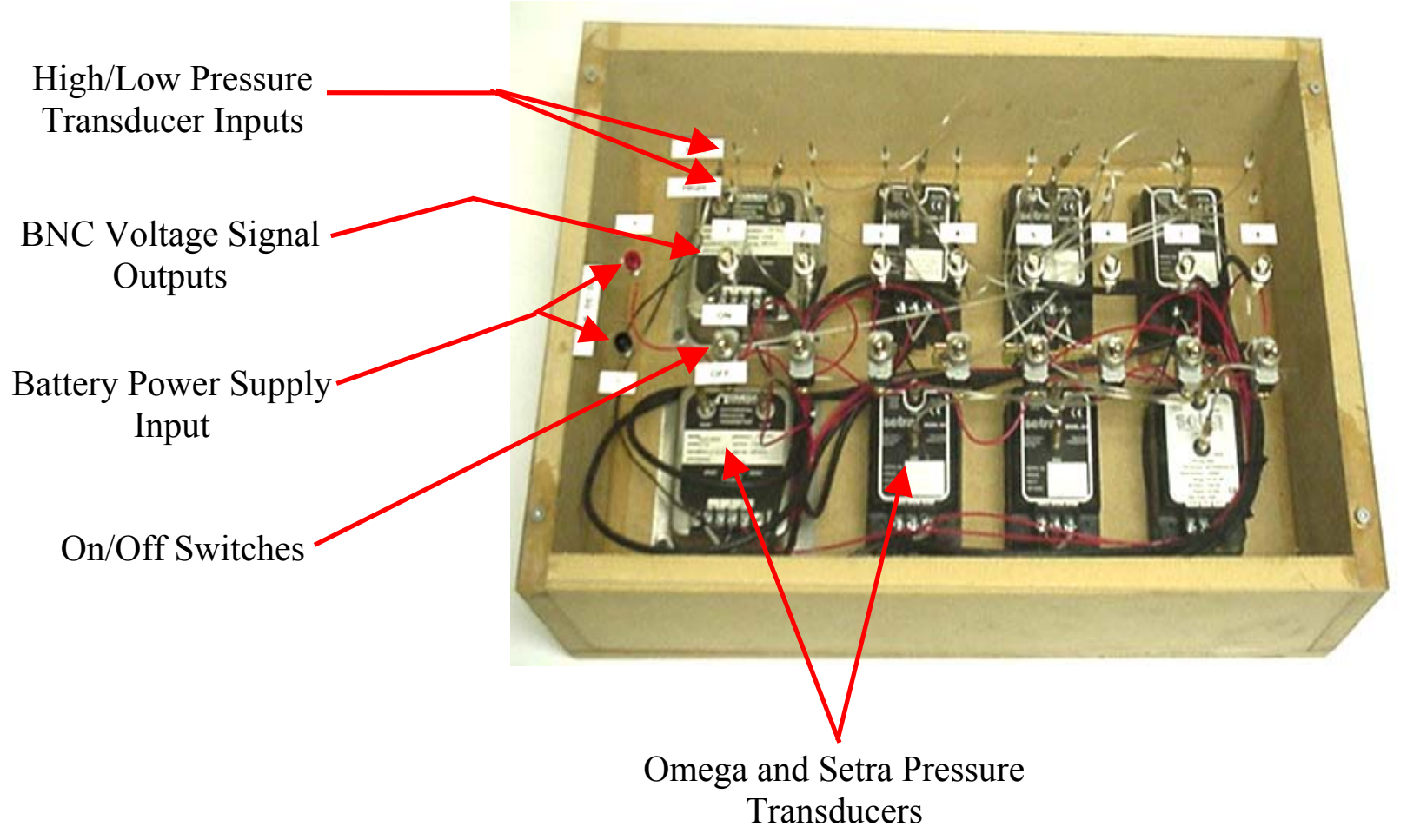

Figure 3.7 Detailed picture of the pressure transducer box. 

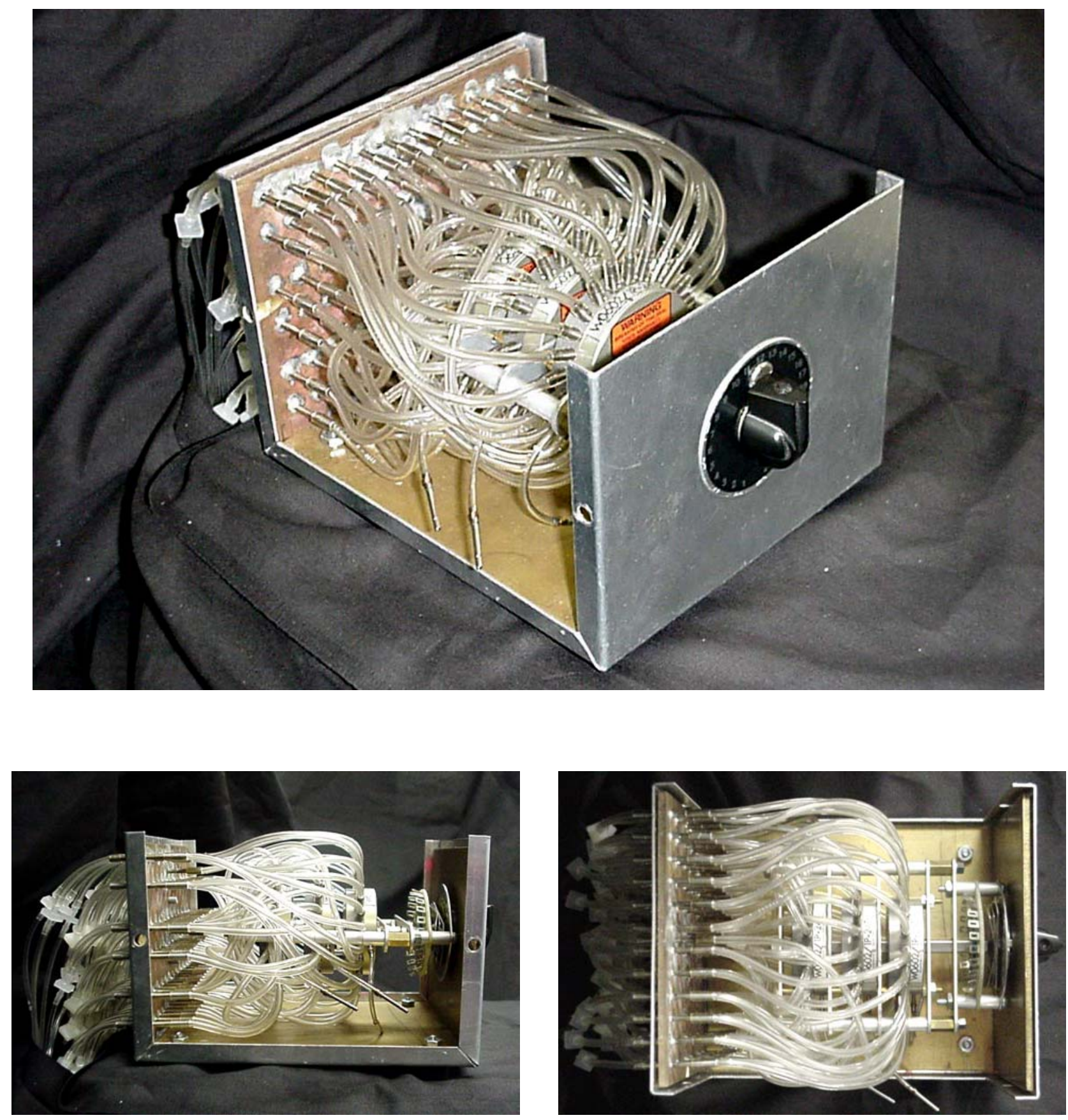

Figure 3.8 Isometric, side and top pictures of the 24-channel, three-wafer scanivalve. 


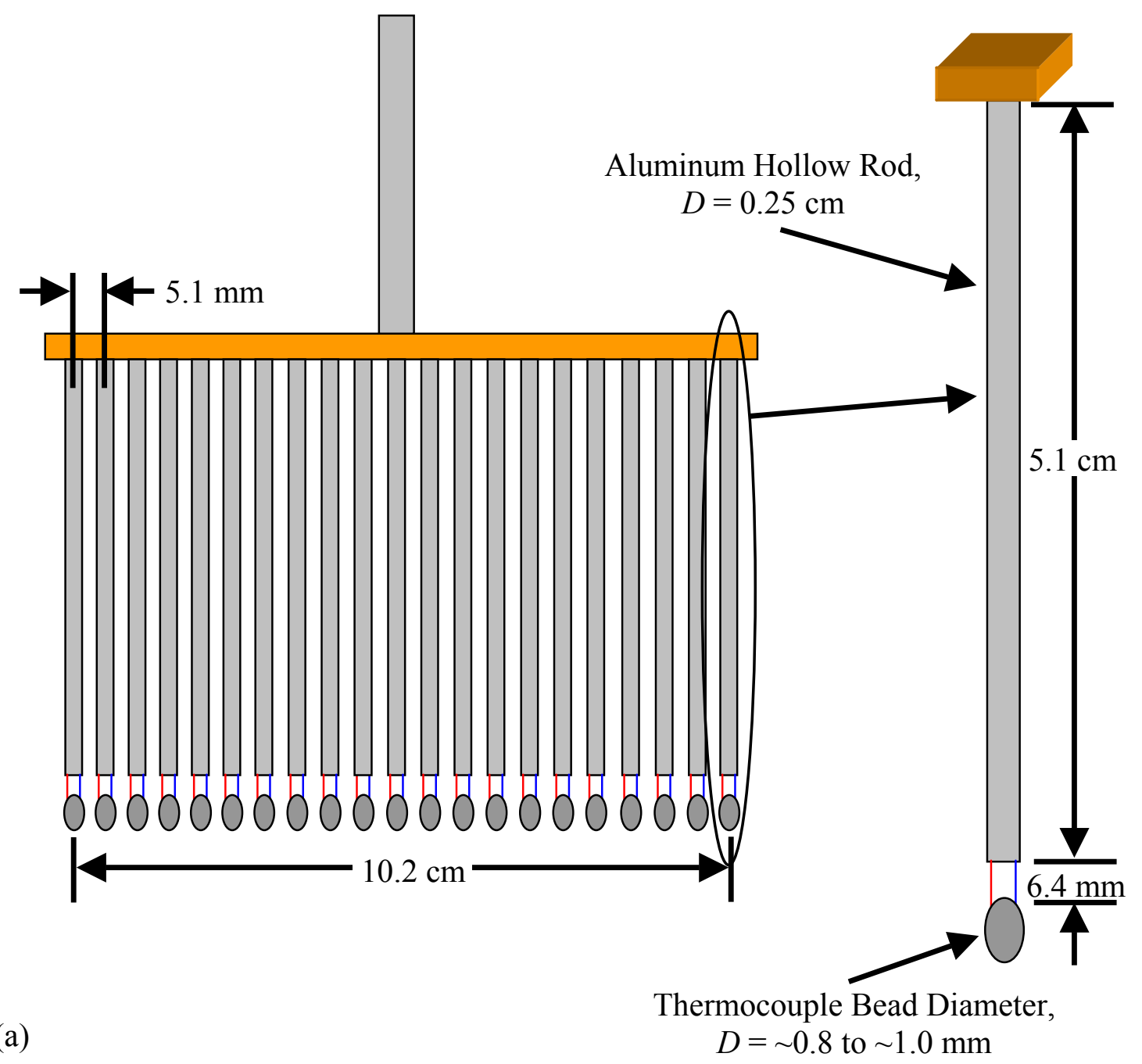

(b)

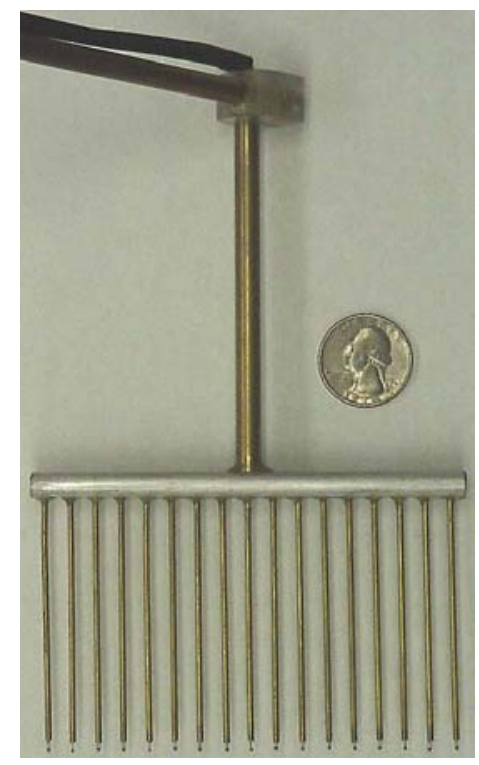

Figure 3.9 (a) Detailed view of the 21-probe, type E thermocouple rake and (b) a photo of the 17-probe, type E thermocouple rake. 


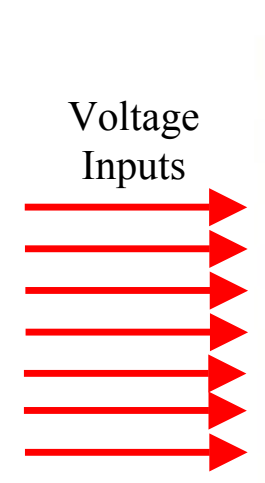

(a)
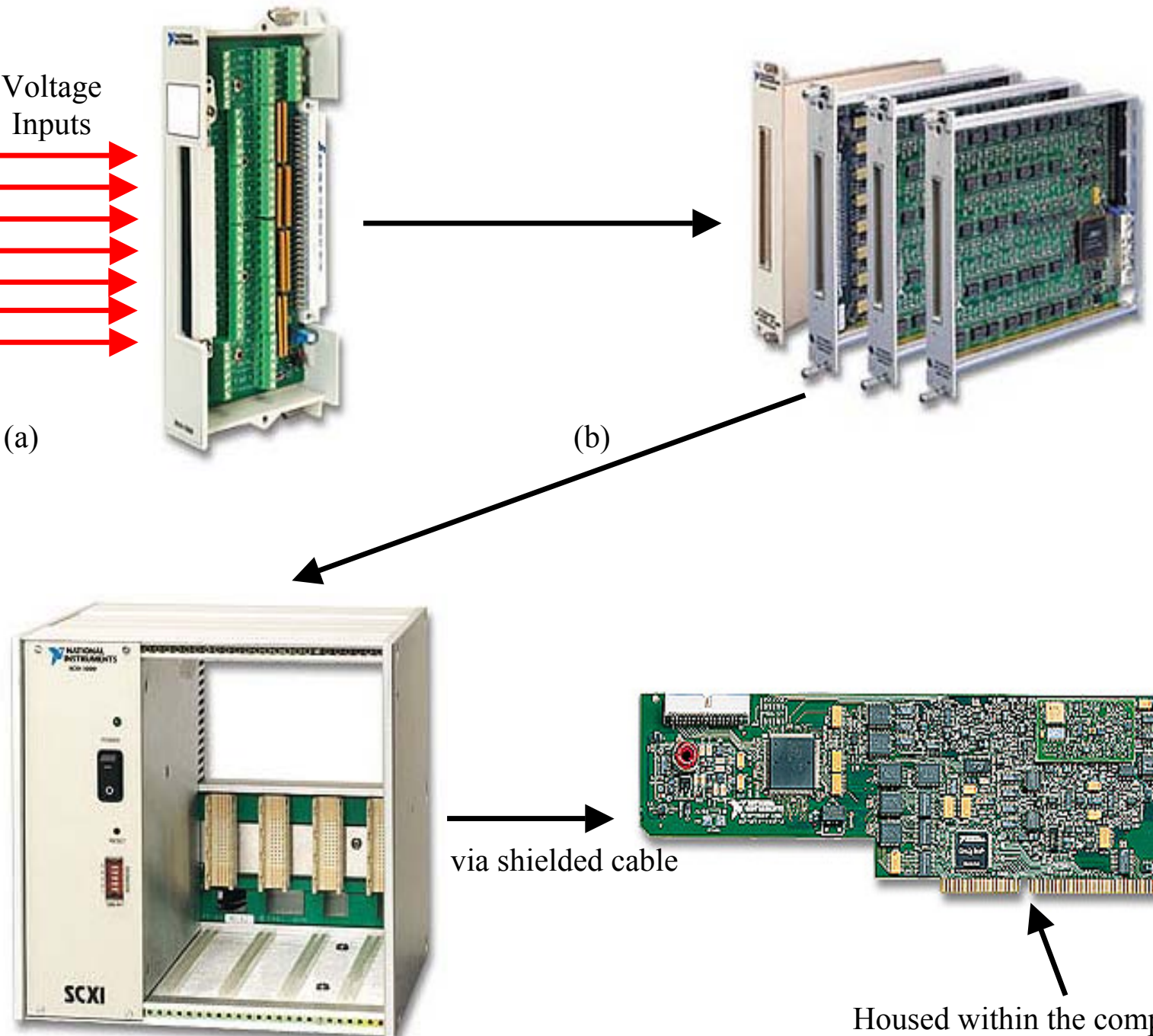

(c)

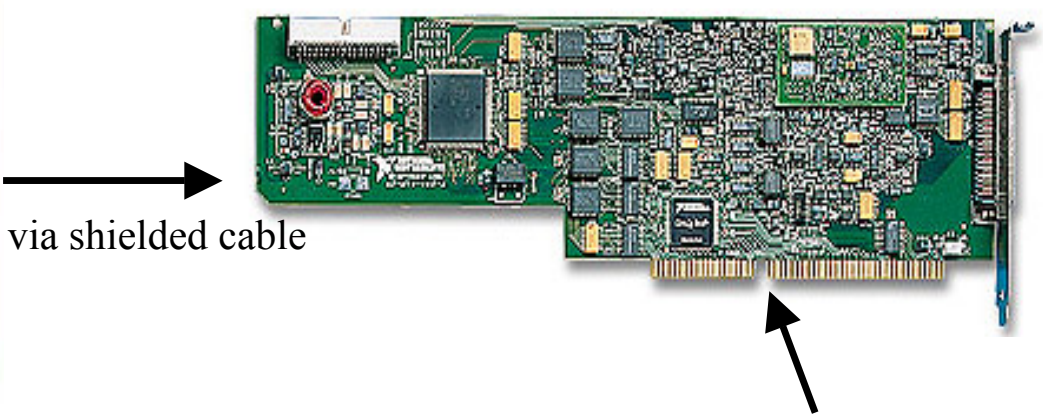

Housed within the computer.

(d)

Figure 3.10 Data acquisition system including the (a) SCXI-1303 terminal block, (b) SCXI-1100 module, (c) SCXI-1000 chassis, and (d) NI AT-MIO-16E-2 DAQ card (National Instruments, 2002). 


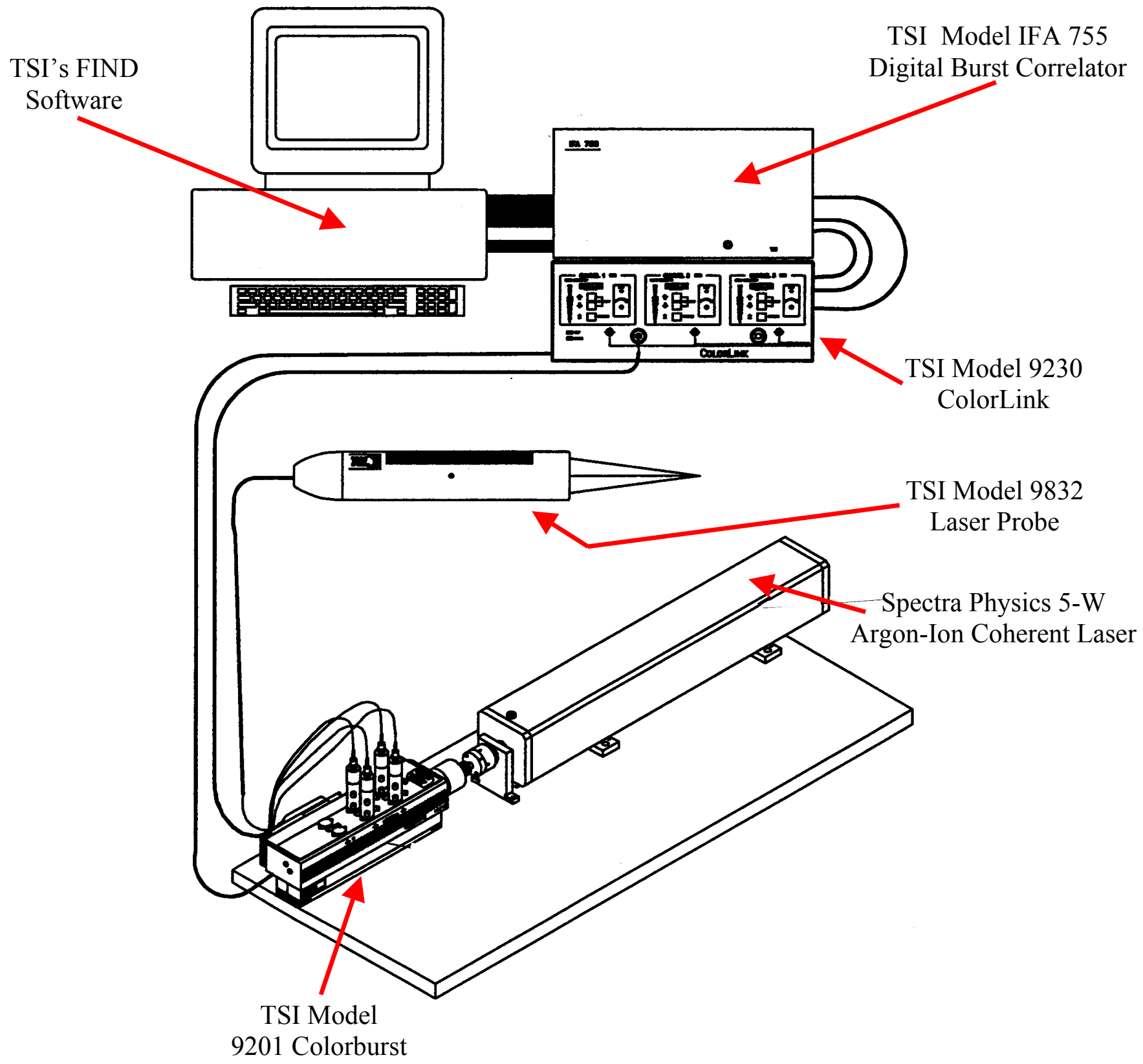

Figure 3.11 Overall laser Doppler velocimeter for a two-component system (TSI, 1995). 


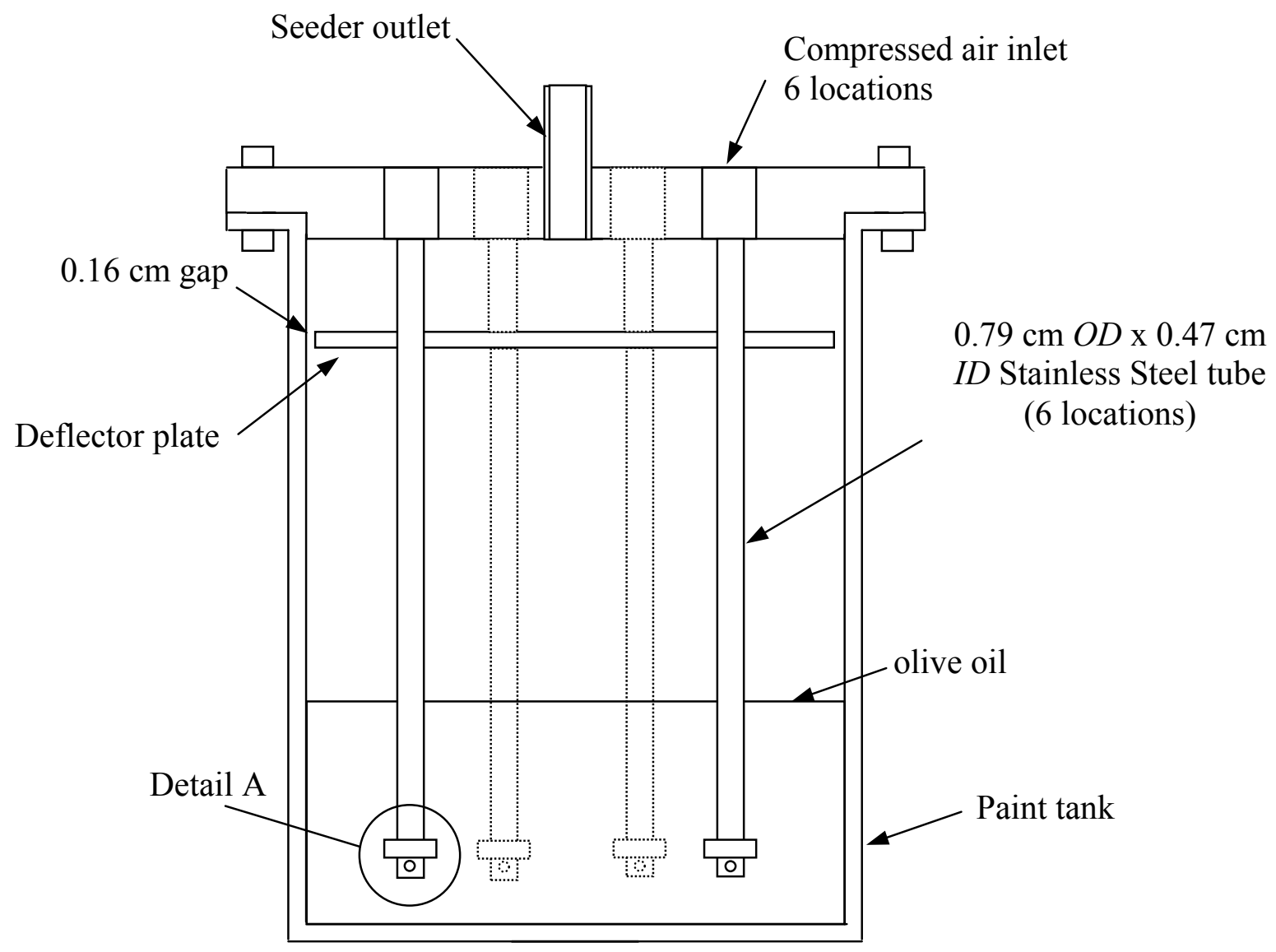

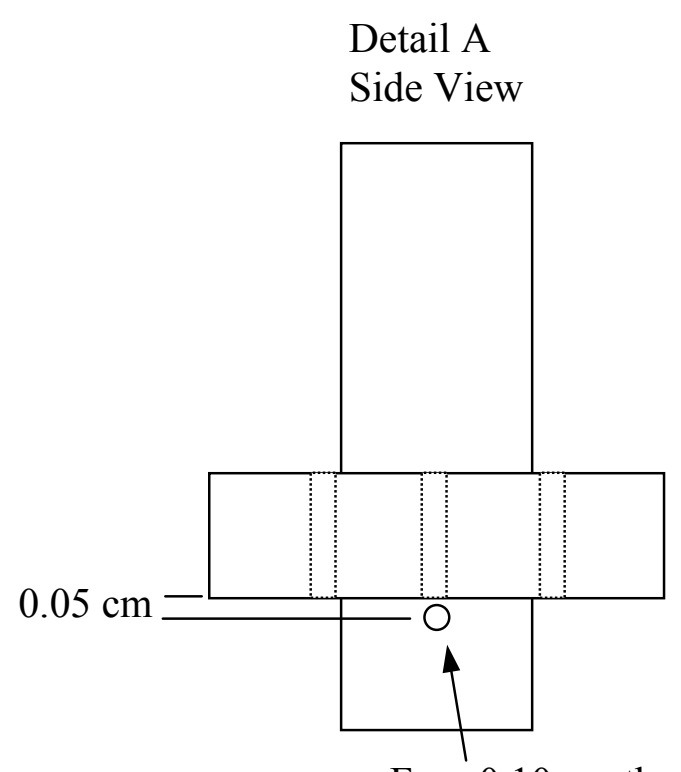

Four $0.10 \mathrm{~cm}$ thru-holes 90-degrees apart
Detail A Top View

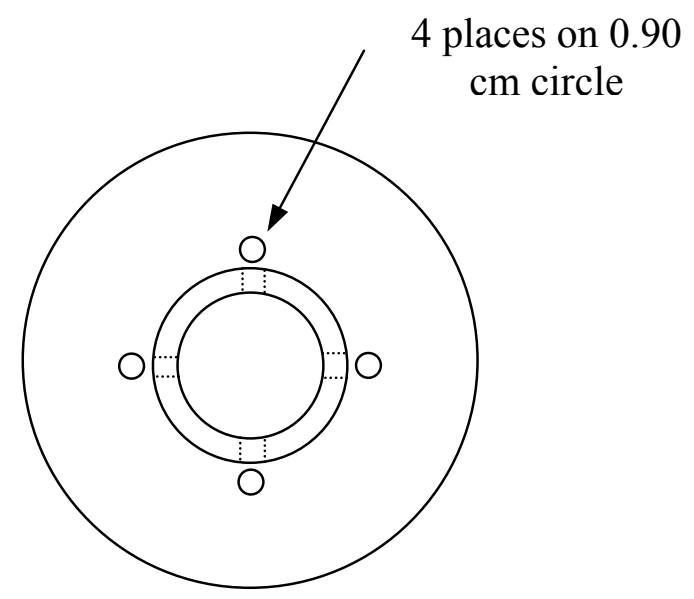

Holes in tube and disk must be aligned

Figure 3.12 Atomized olive oil seed generator used in LDV measurements (Radomsky, 2000). 


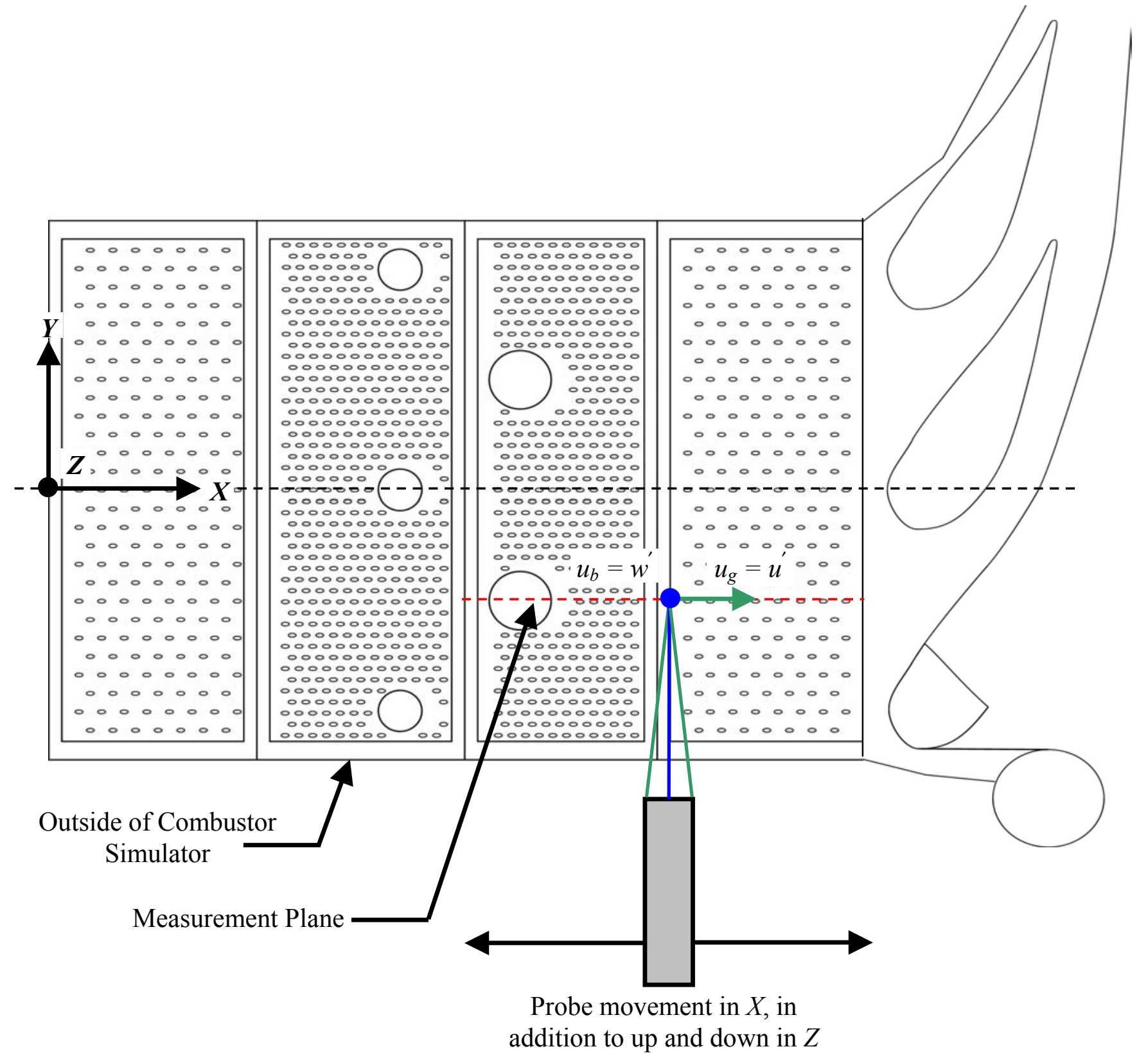

Figure 3.13 Two-component LDV system set-up and orientation in regards to the combustor simulator. 


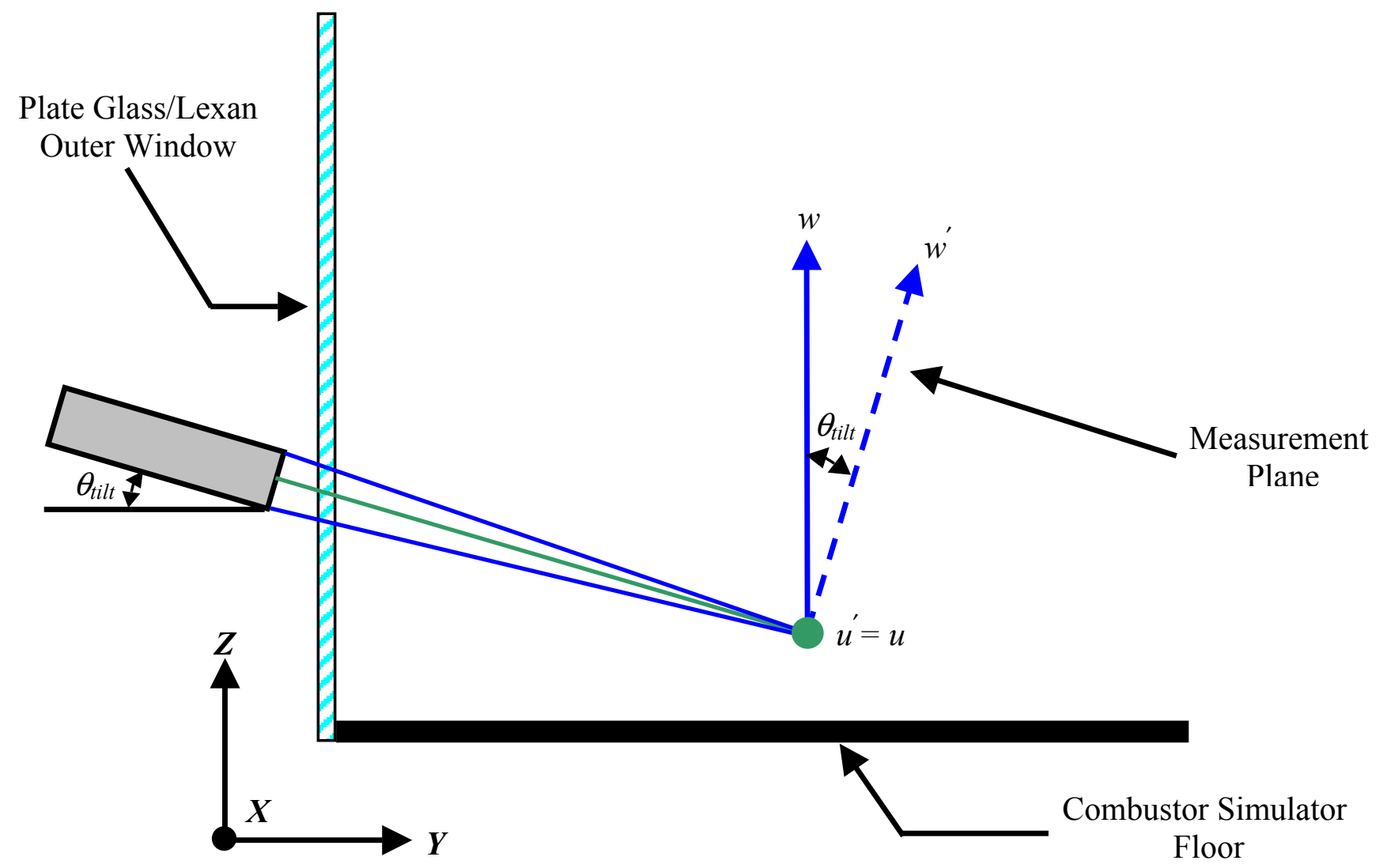

Figure 3.14 Two-component velocity transformation diagram. 

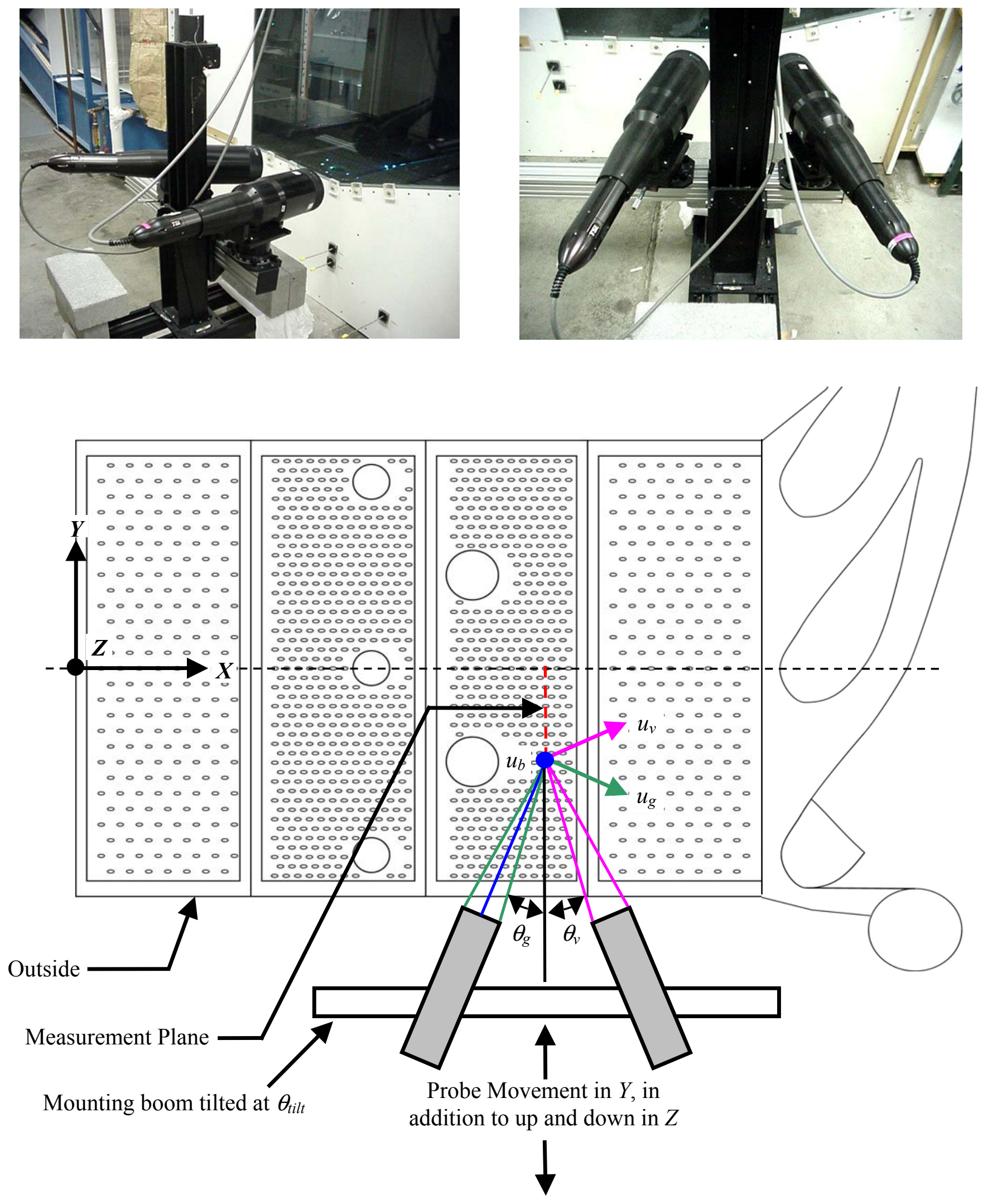

Figure 3.15 Three-component LDV system set-up and orientation in regards to the combustor simulator. 


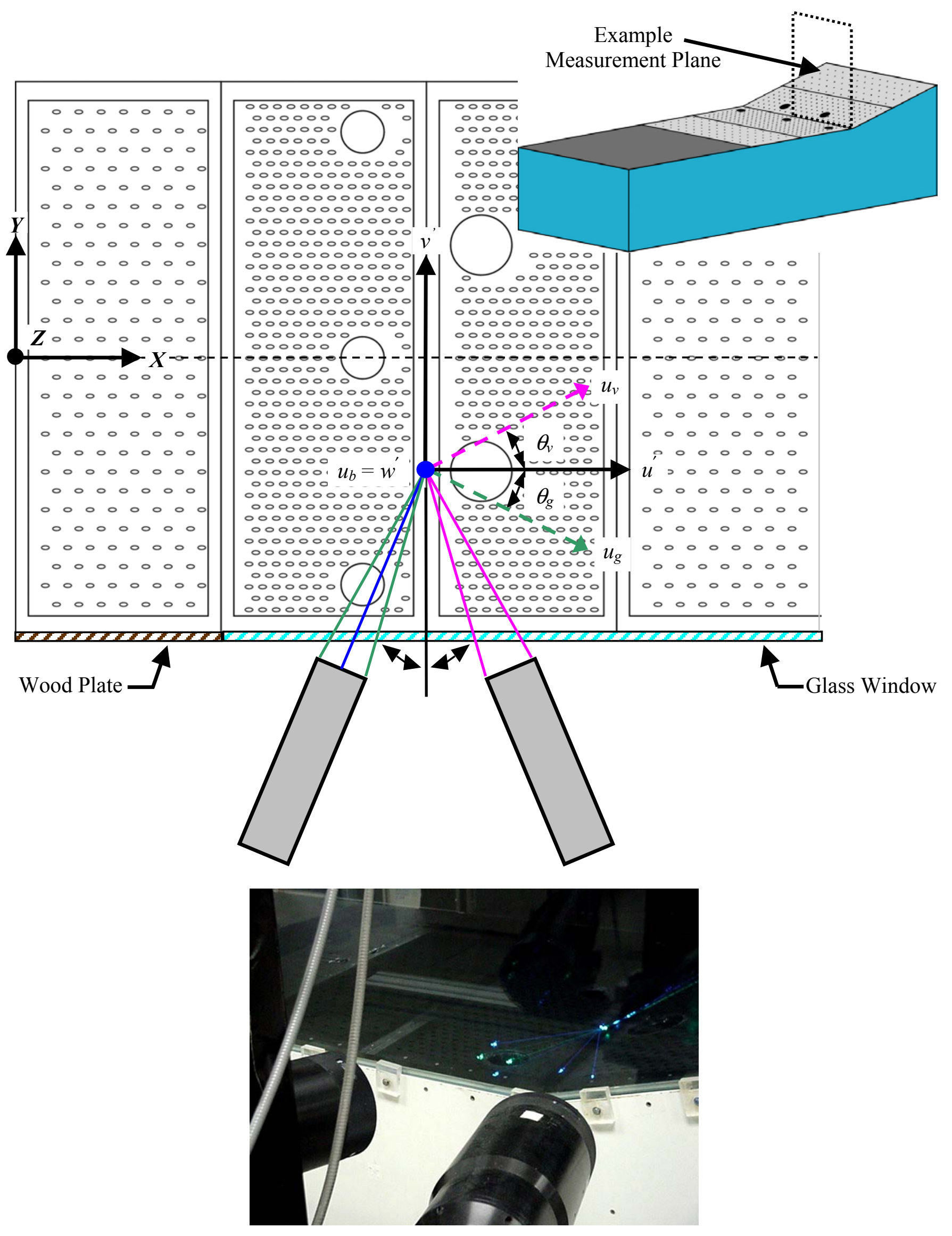

Figure 3.16 Three-component non-orthogonal to orthogonal transformation diagram. 


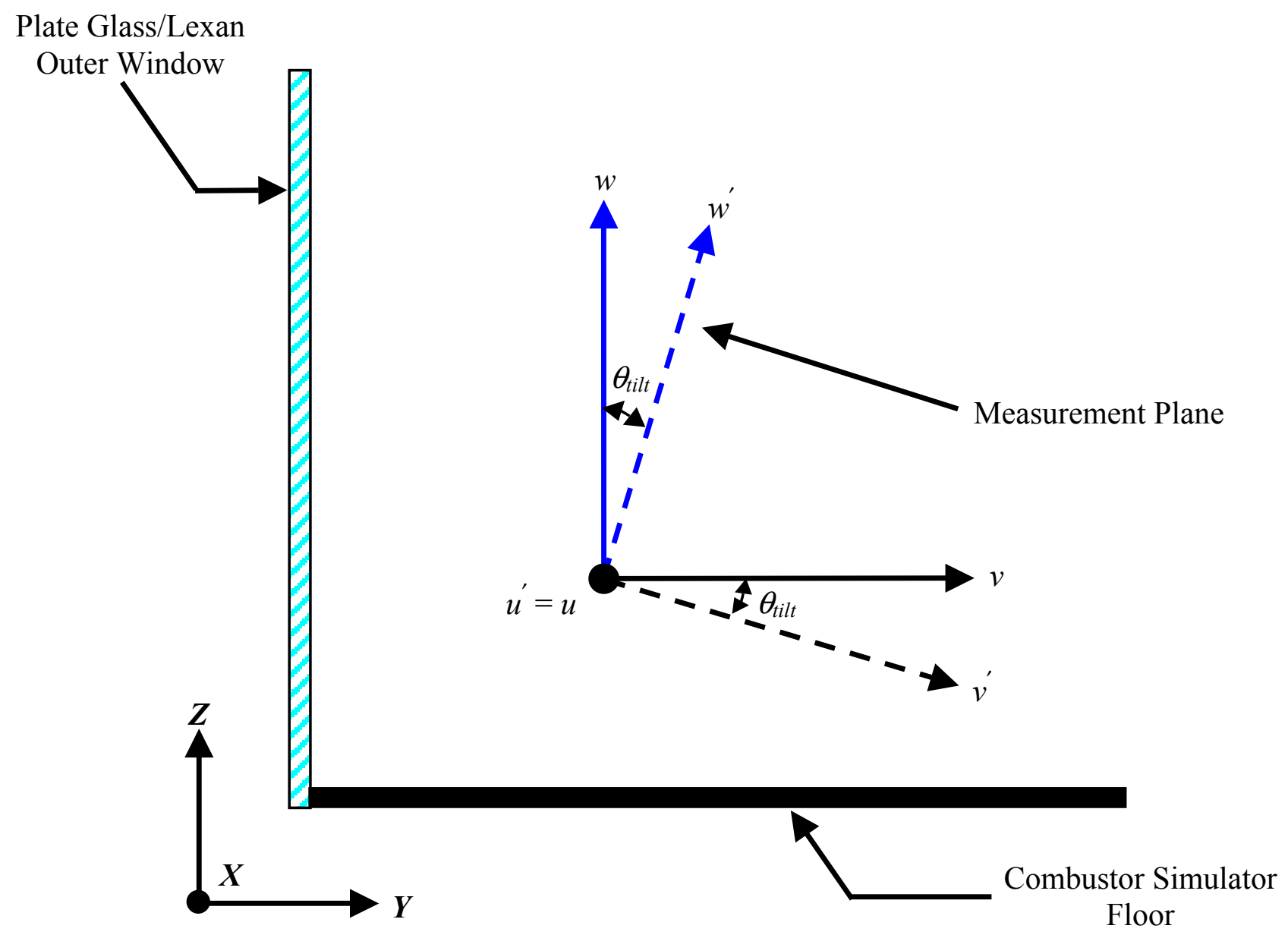

Figure 3.17 Three-component tilt transformation diagram. 


\section{Chapter 4: Experimental Test Matrix}

Defining a proper test matrix was a necessity to accomplish the goals set forth at the beginning of this study. The test matrix included the specific locations, flow conditions, and measurements to be taken in the characterization of the film-cooling and dilution flow of the combustor simulator. This chapter is divided into three subsections. The first section describes the calculations behind setting the various flow conditions within the combustor. The second section illustrates the various measurement planes taken and defines the global coordinate system. Lastly, measured variables and normalization techniques are defined.

\subsection{Combustor Simulator Flow and Thermal Conditions}

In designing the combustor simulator studied here, the purpose was to match relevant flow parameters of an actual aero-engine combustor. However, in setting these parameters, a number of choices needed to be made. An obvious example is the countless number of mass flow rates that can be chosen for the film-cooling and dilution

flow. For our particular combustor, a scaling factor of nine was chosen and the Reynolds number at the exit, as well as the acceleration parameter, $K$, within an actual combustor were matched to determine the overall geometry of the combustor. Coolant flow conditions were determined by matching the momentum flux ratio, $I$, of the film-cooling and dilution holes. The momentum flux ratio was chosen over the velocity ratio because of many studies in literature showing that the trajectory of a jet in crossflow is strongly correlated with the momentum flux ratio. The momentum flux ratio is the ratio of the jet momentum to that of the freestream flow. This is quantitatively described in the following equation:

$$
I=\frac{\rho_{j e t} \cdot u_{j e t}^{2}}{\rho_{\infty} \cdot u_{\infty}^{2}}
$$


where $\rho_{j e t}$ and $\rho_{\infty}$ are the jet and mainstream air densities, while $u_{j e t}$ and $u_{\infty}$ are the jet and local mainstream velocities. Lastly, since the combustor simulator used here was not a reacting combustor, the typically high density ratios of an actual combustor could not be matched. Instead, a density ratio of 1.12 was simulated as opposed to the typical $D R=3$ for an aero-engine combustor.

The momentum flux ratios for the film-cooling panel flows were set within the combustor by measuring the difference between the total pressure within the supply plenum and the external static pressure on top of the liner-panel. For the dilution holes, this measurement proved to be extremely difficult and is discussed in further detail at the end of this section. In setting the dilution flow, pitot-tubes placed at the dilution hole exits were used to adjust the momentum flux ratios of the dilution jets.

To fully understand how the coolant settings were achieved, it is necessary to show how the momentum flux, $I$, is related to the total-static pressure difference. Applying Bernoulli's equation to a pathline that travels from within a supply plenum to the exit of a film-cooling hole results in equation 4.2:

$$
\left(P_{\text {plenum }}-P_{\text {static }}\right)=\Delta P=\frac{1}{2} \rho_{\text {jet }}\left(u_{\text {jet }, \text { ideal }}\right)^{2}
$$

where $u_{\text {jet,ideal }}$ is the theoretical velocity of the flow exiting the film-cooling hole.

The discharge coefficient, equation 4.3 , is defined as the ratio of the actual mass flow rate to the theoretical mass flow rate.

$$
C_{d}=\frac{m_{\text {actual }}}{m_{\text {theoretical }}}
$$

In this case, the actual mass flow rate was that which was desired experimentally, while the theoretical mass flow rate was calculated from Bernoulli's equation. Previous experiments reported by Barringer (2001) fully documented how these discharge coefficients were achieved. In short, however, to experimentally determine the discharge coefficients, a $5 \mathrm{Hp}$ fan was used to direct coolant flow through a laminar flow element 
into a large plenum chamber directly attached to a film-cooling panel. The measured experimental quantities included the flow rate across the laminar flow element, the total pressure within the plenum, and the pressure at the exit of the holes. This data allowed for the actual mass flow rate to be deduced, while Bernoulli's equation gave the theoretical mass flow rate. Applying the relationship in equation 4.3, the discharge coefficient was then calculated.

Substituting the expressions for the respective mass flow rates into equation 4.3 results in

$$
C_{d}=\frac{N \cdot \rho_{\text {jet }} \cdot A_{\text {hole }} \cdot u_{\text {jet }}}{N \cdot \rho_{\text {jet }} \cdot A_{\text {hole }} \cdot u_{\text {jet }, \text { ideal }}}
$$

where $N$ is the number of film-cooling holes within the panel and $A_{\text {hole }}$ is the crosssectional area of each hole. Canceling the common terms and re-writing expression 4.4 allows one to relate the actual film-cooling jet velocity to the theoretical exit velocity as

$$
u_{j e t}=C_{d} \cdot u_{\text {jet }, \text { ideal }}
$$

Substituting equation 4.5 into Bernoulli's equation (4.2) results in the true total-to-static pressure difference needed:

$$
\left(P_{\text {plenum }}-P_{\text {static }}\right)=\Delta P=\frac{1}{2} \rho_{\text {jet }}\left(\frac{u_{\text {jet }, \text { ideal }}}{C_{d}}\right)^{2}
$$

Recalling that equation 4.1 defined the momentum flux ratio, solving this for the actual jet velocity and substituting into equation 4.6 results in expression 4.7 , which relates the pressure difference to the momentum flux, $I$ :

$$
\Delta P=\frac{I \cdot u_{\infty}^{2} \cdot \rho_{\infty}}{2 \cdot C_{d}^{2}}
$$


From equation 4.7, all of the desired quantities are initially known at the combustor inlet and the inlet velocity, $u_{\infty}$, was measured using an LDV. A summary of the number of holes in each liner-panel and dilution row, as well as their respective discharge coefficients and diameters can be found in Table 4.1.

The calculation of the required pressure drop to achieve the desired momentum flux in the first liner panel was straightforward. However, determining the required pressure drops for the remaining three panels and two rows of dilution was a slightly more involved process.

As the flow progressed down the combustor, the average velocity was constantly increasing due to added mass flow from the combustor liner panels and dilution, as well as the area contraction. Consequently, when determining the required pressure differences to match the desired momentum flux ratios, one cannot simply use the freestream velocity measured at the inlet. The inlet freestream velocity was only valid for the first set of liner panels. A mass-averaged velocity was used for the remaining liner panels and rows of dilution. The mass-averaged velocity was defined at the leading edge of each liner-panel and was directly calculated from the inlet mass flow rate plus any additional mass flow across the entire combustor width. For example, the mass flow rate at the leading edge of liner panel three was the inlet mass flow plus the mass flow added by the first and second liner panels as well as the first row of dilution jets.

To illustrate this quantitatively, consider the desired quantity to be the freestream mass-averaged velocity at the leading edge of liner panel two. First, the velocity exiting each of the film-cooling holes must be determined. Knowing the required momentum flux out of the liner-panels, the velocity exiting each film-cooling hole may be deduced from equation 4.1. The total mass-flow out of panel one is given by

$$
m_{1}=N \cdot \rho_{j e t} \cdot A_{\text {hole }} \cdot u_{j e t}
$$

With the mass flow rate, $m_{1}$, out of the first liner-panel known, twice that quantity (top and bottom panels) was added to the mass flow rate at the inlet of panel one, which is given by equation 4.9 


$$
m_{\text {inlet }}=\rho_{\infty} \cdot A_{c} \cdot u_{1}=\rho_{\infty} \cdot A_{c} \cdot u_{\infty}
$$

where $A_{c}$ is the cross-sectional area of the combustor at the leading edge of the panel. At the leading edges of panels 1,2 , and $3, A_{c}$ was $1.11 \mathrm{~m}^{2}$, while at the leading edge of panel 4 and the combustor exit, the cross-sectional areas were $0.88 \mathrm{~m}^{2}$ and $0.62 \mathrm{~m}^{2}$, respectively. The total mass flow rate at the start of panel two may now be determined by adding the results of equations 4.8 and 4.9 together. Using equation 4.10 , the massaveraged velocity at the inlet of panel two was determined:

$$
u_{2}=\frac{m_{\text {inlet }}+m_{1}}{\rho_{\infty} \cdot A_{c}}
$$

This repetitive process was repeated at the leading edge of panels three and four. In all, the proper mass-averaged velocities lead to the required pressure difference in equation 4.7 to be set within each plenum. A graphical representation of the relevant massaveraged planes, as well as a graph showing the qualitative variation in the freestream velocity and the mass-flow rates can be found in Figure 4.1.

Lastly, as mentioned in Chapter 3, since the size of the dilution holes was relatively large in comparison to its plenum size, space constraints did not allow for the construction of a plenum large enough to permit the flow to stagnate. Therefore, it was inaccurate to take a total pressure reading, $P_{\text {plenum }}$, inside of the plenum and use this along with the static pressure, $P_{\text {static }}$, on the external side of the panels, to set the mass flow rate through the dilution rows. Instead, a more accurate method was to use a pitot-tube to set the required velocity, thus achieving the necessary mass flow. The pressure drop read across the pitot-tube is given by

$$
\Delta P=\frac{u_{j e t}^{2} \cdot \rho_{j e t}}{2}
$$


where again, the desired jet velocity, $u_{j e t}$, was determined by equation 4.1 . Recall that equation 4.1 is the desired momentum flux ratio of the jet to that of the local freestream.

\subsection{Measurement Planes}

As mentioned previously, the goals of this study were to understand the complex thermal and velocity flow fields within the combustor. Another objective was to compare experimental results with those results computationally predicted by Stitzel (2001). For this reason, the selection of the proper measurement planes was an important aspect of this study.

For this study, it was important to establish a global coordinate system within the combustor to keep the measurement locations within the same frame of reference. The origin was selected at the mid-pitch of the first combustor liner panel on the bottom of the simulator and can be seen in Figure 4.2. The streamwise direction was denoted by $X$, the pitchwise direction denoted by $Y$, and the span by $Z$. Lastly, all of the locations were non-dimensionalized by their respective characteristic lengths within the combustor, which included the following: the combustor length, $L$, was used for the streamwise direction; the sector width (two vane pitches), $W$, was used for the pitch; and the combustor inlet height, $H_{\text {in }}$, for the span.

The thermal measurement planes included five different locations in the combustor simulator. Plane 0p (pitchwise), shown in Figure 4.3, was selected directly downstream of the first combustor film-cooling liner panel and was located two filmcooling hole diameters $(15.2 \mathrm{~mm})$ from the downstream edge of the last row of holes at $X / L=0.24$. The choice for this plane was to quantify the effects of varying the filmcooling momentum flux. The width of the measurement plane covered a minimum of five to a maximum of eight film-cooling holes. Secondly, plane $1 \mathrm{p}$ was selected immediately downstream of the first row of dilution to quantify the film cooling interaction with dilution flow as well as the interaction of the dilution jets with each other. Plane $1 \mathrm{p}$ extended across one full-pitch in width and to the combustor mid-span in 
height. This plane was located two film-cooling hole diameters $(15.2 \mathrm{~mm})$ from the downstream edge of the last row of holes in panel $2(X / L=0.50)$.

One might ask, what was the standard by which the remaining measurement planes were selected? The answer lies in a model proposed by Sgarzi and Leboeuf (1997). Figure 2.28 shows this model and its key features. The partial motivation in selecting the remaining planes was to try and capture the thermal effects of these vortical structures appearing in and around the jet in crossflow. Planes 1s and 2s (streamwise), shown in Figure 4.3, were selected to characterize the streamwise behavior of the first and second rows of dilution jets. Plane $1 \mathrm{~s}$ was located directly at mid-pitch $(Y / W=0)$ and encompassed an area that went from a $X / L$ location of 0.35 to 0.50 and extended slightly higher than the mid-span. The thermal plane $2 \mathrm{~s}$ was located at a $Y / W$ of 0.25 and covered from $X / L=0.46$ to $X / L=1.02$. The measurements in plane $2 \mathrm{~s}$ also extended slightly above the mid-span. Lastly, plane $2 \mathrm{p}$ was located one diameter (second row dilution hole) downstream of the trailing edge of a second row dilution hole. The intent of this plane was to characterize the downstream interaction of the first and second rows of dilution. The streamwise location of this cross-pitch plane was $X / L=0.69$. Plane $2 \mathrm{~s}$ covered from $Y / W=-0.10$ to $\mathrm{Y} / \mathrm{W}=0.34$ and extended slightly above the midspan. A summary of the extent of the thermal measurement planes and their locations can be found in Table 4.2.

As stated previously, the selection of the measurement planes was partially driven by Sgarzi and Leboeuf's model of a jet in crossflow. In the thermal fields, the aim was to see the effects of the various vortices on the dilution flow and the film-cooling. In the flow field planes, the visualization of these specific vortices was the primary ambition. In all, a complete thermal and velocity data set was to be acquired.

Only planes $1 \mathrm{p}, 2 \mathrm{p}$, and $2 \mathrm{~s}$ were taken with the LDV. Plane $1 \mathrm{p}$, located approximately two film-cooling hole diameters $(15.2 \mathrm{~mm})$ downstream from the trailing edge of the last row of film-cooling, was intended to capture the horseshoe, half-wake, and counter-rotating vortex pair seen in Figure 2.28. Likewise, plane $2 p$ was chosen to show the interaction of the counter-rotating vortex pairs from dilution one and two, while the streamwise plane 2 s would depict the lip, half-wake, or half-wall vortices. Unlike the temperature planes, the two cross-pitch planes, $1 \mathrm{p}$ and $2 \mathrm{p}$, were acquired on the basis of 
symmetry. Moreover, instead of taking plane 2 s at a $Y / W$ of -0.25 , it was done at a $Y / W$ of -0.25 , since this allowed optical access. Table 4.3 summarizes the sizes and locations and Figure 4.4 provides a visualization of the flow fields within the combustor.

\subsection{Measured Quantities and Definitions}

To understand the various thermal and flow field results presented in Chapter 5, the numerous non-dimensional quantities must be defined. The first part of this study consisted of numerous thermal measurements within the combustor. Conditions such as the coolant or inlet temperatures varied daily. For example, changes in the daily tap water or temperature of the laboratory could cause inlet and coolant temperatures to vary as much as $3-8{ }^{\circ} \mathrm{C}$ from day to day. To allow for the direct comparison of thermal field results throughout the combustor with each other, a non-dimensional temperature was defined. The definition for the non-dimensional temperature, $\theta$, is given as

$$
\theta=\frac{T_{\infty}-T}{T_{\infty}-T_{c}}
$$

Here, $T$ is the local measured temperature, and $T_{\infty}$ and $T_{c}$ are the combustor inlet freestream and coolant temperatures, respectively. It is important to note, with the convention used in equation 4.12, a value of zero represents the hottest temperatures while a value of one represents the coldest temperatures. Also, the coolant temperature, $T_{c}$, was measured in the plenum intrinsic to the specific plane of measurement.

Although the velocity was not as irregular as the temperature on a day-to-day basis, non-dimensional definitions were equally important in providing a direct comparison between measurement locations. To begin, all mean velocity quantities along with their corresponding root-mean-square (rms) levels for the velocity fluctuations were normalized by the inlet freestream velocity, $u_{i n}$. The probability of obtaining a sample was proportional to the speed of the flow. As a result, statistical particle bias corrections were applied to the data by weighting each individual sample based on the 
residence time of a particle in the probe volume. The definitions for the normalized mean and normalized rms levels are given below in equations 4.13 and 4.14, respectively:

$$
\begin{aligned}
& \frac{\bar{u}}{u_{i n}}=\frac{\left(\frac{\sum_{i=1}^{n} u_{i} \cdot \tau_{i}}{\left.\sum_{i=1}^{n} \tau_{i}\right)}\right.}{u_{i n}} \\
& \frac{u_{r m s}}{u_{i n}}=\frac{\sqrt{\left(\frac{\sum_{i=1}^{n} u_{i}^{2} \cdot \tau_{i}}{\sum_{i=1}^{n} \tau_{i}}-\bar{u}^{2}\right)}}{u_{i n}}
\end{aligned}
$$

Here, $u_{i}$ is the actual velocity sample, $\tau$ is corresponding residence time of that particle in the probe volume, and $n$ is the total number of samples taken (TSI, 2000). These definitions have been defined for the streamwise velocity component, $u$, however, the same definitions apply for the pitchwise and vertical velocity components, $v$ and $w$. A normalized combustor turbulent kinetic energy, $\mathrm{k} / \mathrm{u}_{i n}^{2}$, was then defined as

$$
\frac{k}{u_{i n}^{2}}=\frac{\frac{1}{2}\left(u_{r m s}^{2}+v_{r m s}^{2}+w_{r m s}^{2}\right)}{u_{i n}^{2}}
$$

for the three-component velocity measurements, and

$$
\frac{k}{u_{i n}^{2}}=\frac{\frac{1}{2}\left(u_{r m s}^{2}+w_{r m s}^{2}\right)}{u_{i n}^{2}}
$$

for the two-component velocity measurements. In addition to a normalized turbulent kinetic energy, a combustor turbulence level was also defined. This turbulence level was 
based on the individual rms values at each measurement location and the inlet velocity to the combustor. Equations 4.17 and 4.18 give the turbulence levels for the threecomponent and two-component measurements, respectively:

$$
\begin{aligned}
& T L=\frac{\sqrt{\frac{1}{3}\left(u_{r m s}^{2}+v_{r m s}^{2}+w_{r m s}^{2}\right)}}{u_{i n}} \\
& T L=\frac{\sqrt{\frac{1}{2}\left(u_{r m s}^{2}+w_{r m s}^{2}\right)}}{u_{i n}}
\end{aligned}
$$

Lastly, a local turbulence level was defined using the magnitude of the rms values at each location and dividing them by the local velocity magnitude. The equations for the local turbulence are given below. Equation 4.19 is for the three-component measurements while equation 4.20 is for the two-component measurements:

$$
\begin{aligned}
& T u=\frac{\sqrt{\frac{1}{3}\left(u_{r m s}^{2}+v_{r m s}^{2}+w_{r m s}^{2}\right)}}{\sqrt{u^{2}+v^{2}+w^{2}}} \\
& T u=\frac{\sqrt{\frac{1}{2}\left(u_{r m s}^{2}+w_{r m s}^{2}\right)}}{\sqrt{u^{2}+w^{2}}}
\end{aligned}
$$

In all, these various flow field measurement quantities, particularly the turbulence levels, provide a good means of comparing the experimental results measured within this study to those prevalent in the literature. 
Table 4.1 The Number of Holes in Each Liner-Panel and Dilution Row, Including their Respective Discharge Coefficients and Diameters

\begin{tabular}{|l|c|c|c|}
\hline & Number of Holes & Discharge Coefficient, $\boldsymbol{C}_{\boldsymbol{d}}$ & Diameter (cm) \\
\hline Panel 1 & 202 & 0.739 & 0.76 \\
\hline Panel 2 & 478 & 0.733 & 0.76 \\
\hline Dilution Row 1 & 3 & 0.829 & 8.51 \\
\hline Panel 3 & 413 & 0.733 & 0.76 \\
\hline Dilution Row 2 & 2 & 0.912 & 12.12 \\
\hline Panel 4 & 130 & 0.739 & 0.76 \\
\hline
\end{tabular}


Table 4.2 Sizes and Locations of the Thermal Measurement Planes

\begin{tabular}{|c|c|c|}
\hline Measurement Plane & $\begin{array}{c}\text { Location } \\
\left(\boldsymbol{X} \boldsymbol{L}, \boldsymbol{Y} / \boldsymbol{W}, \boldsymbol{Z} / \boldsymbol{H}_{\text {in }}\right)\end{array}$ & $\begin{array}{c}\text { Size } \\
(\text { Non-Dimensional })\end{array}$ \\
\hline $\begin{array}{c}0 \mathrm{p} \\
(\text { Film-Cooling } I=3)\end{array}$ & $\begin{array}{c}(0.24,0.09,0) \\
\text { Bottom Inside Corner }\end{array}$ & $\begin{array}{c}0.39 \times 0.06 \\
Y / W \times / H_{\text {in }}\end{array}$ \\
\hline $0 \mathrm{p}$ & $(0.24,0.11,0)$ & $0.21 \times 0.06$ \\
$($ Film-Cooling $I=9)$ & Bottom Inside Corner & $Y / W \times Z / H_{\text {in }}$ \\
\hline $1 \mathrm{p}$ & $(0.50,0.26,0)$ & $0.53 \times 0.50$ \\
& Bottom Inside Corner & $Y / W \times Z / H_{\text {in }}$ \\
\hline $1 \mathrm{~s}$ & $(0.35,0,0)$ & $0.15 \times 0.56$ \\
& Upstream Bottom Corner & $X / L \times Z / H_{\text {in }}$ \\
\hline $2 \mathrm{p}$ & $(0.69,0.10,0.08)$ & $0.44 \times 0.52$ \\
& Bottom Inside Corner & $Y / W \times Z / H_{\text {in }}$ \\
\hline $2 \mathrm{~s}$ & $(0.46,0.25,0)$ & $0.56 \times 0.56$ \\
& Upstream Bottom Corner & $X / L \times Z / H_{\text {in }}$ \\
\hline
\end{tabular}

Table 4.3 Sizes and Locations of the Flow Field Planes

\begin{tabular}{|c|c|c|c|}
\hline $\begin{array}{c}\text { Measurement } \\
\text { Plane }\end{array}$ & $\begin{array}{c}\text { Location } \\
\left(\boldsymbol{X} / \mathbf{L}, \boldsymbol{Y} / \boldsymbol{W}, \boldsymbol{Z} / \boldsymbol{H}_{\text {in }}\right)\end{array}$ & $\begin{array}{c}\text { Size } \\
(\text { Non-Dimensional })\end{array}$ & $\begin{array}{c}\text { LDV } \\
\text { Component }\end{array}$ \\
\hline Plane $1 \mathrm{p}$ & $\begin{array}{c}(0.50,0,0) \\
\text { Bottom Inside Corner }\end{array}$ & $\begin{array}{c}0.50 \times 0.50 \\
Y / W \times Z / H_{i n}\end{array}$ & 3 \\
\hline Plane $2 \mathrm{p}$ & $(0.69,0,0.08)$ & $0.25 \times 0.43$ & 3 \\
& Bottom Inside Corner & $Y / W \times Z / H_{\text {in }}$ & \\
\hline Plane 2s & $(0.46,-0.25,0)$ & $0.47 \times 0.50$ & 2 \\
& Upstream Bottom Corner & $X / L \times Z / H_{\text {in }}$ & \\
\hline
\end{tabular}


(a)
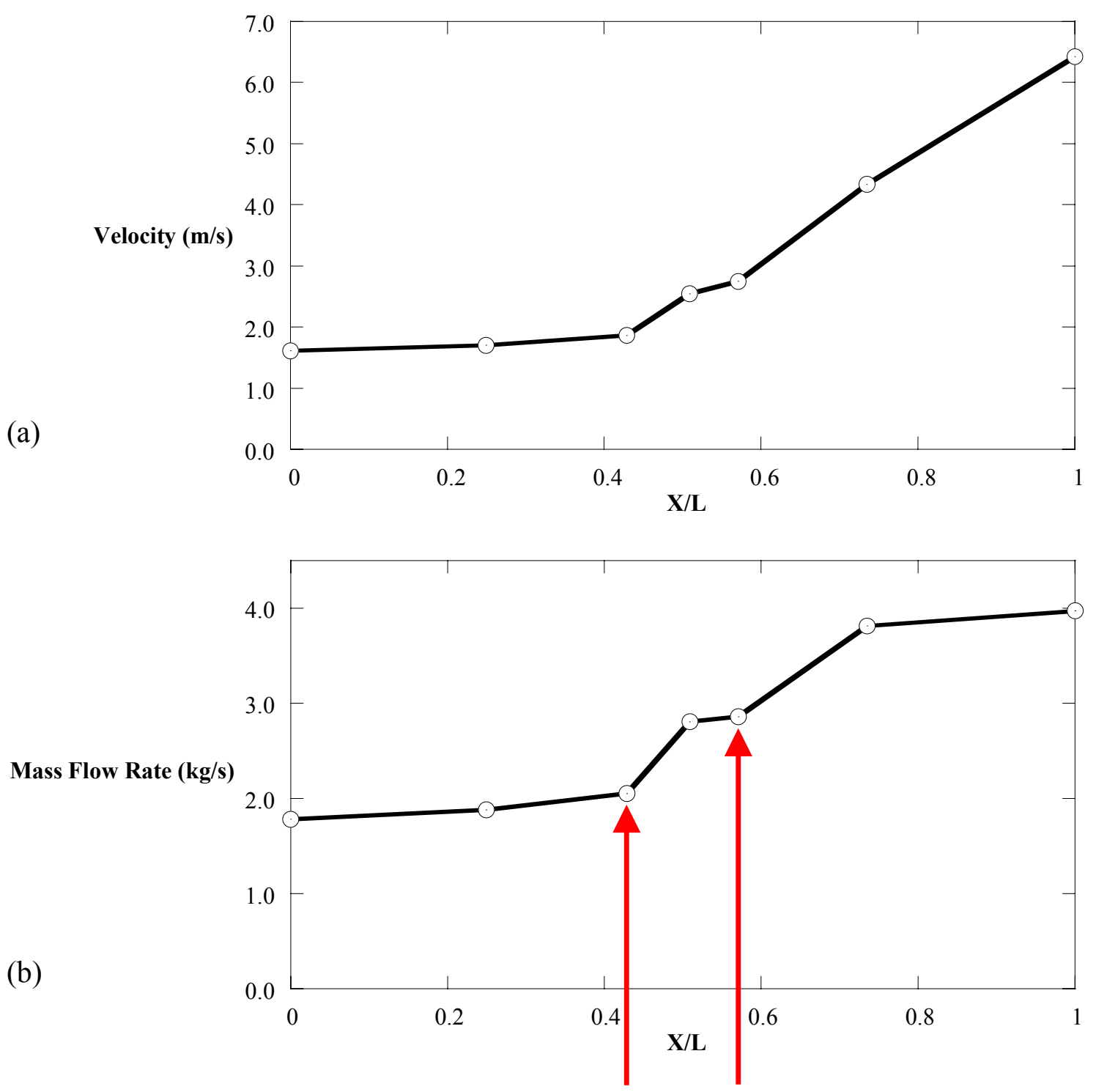

Dilution Injection

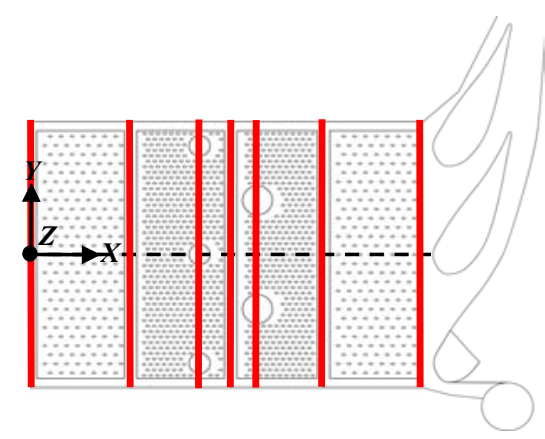

Figure 4.1 A graphical representation of the mass-averaged planes with graphs showing the variations in (a) velocity and (b) mass flow rates along the combustor simulator for a typical run. 


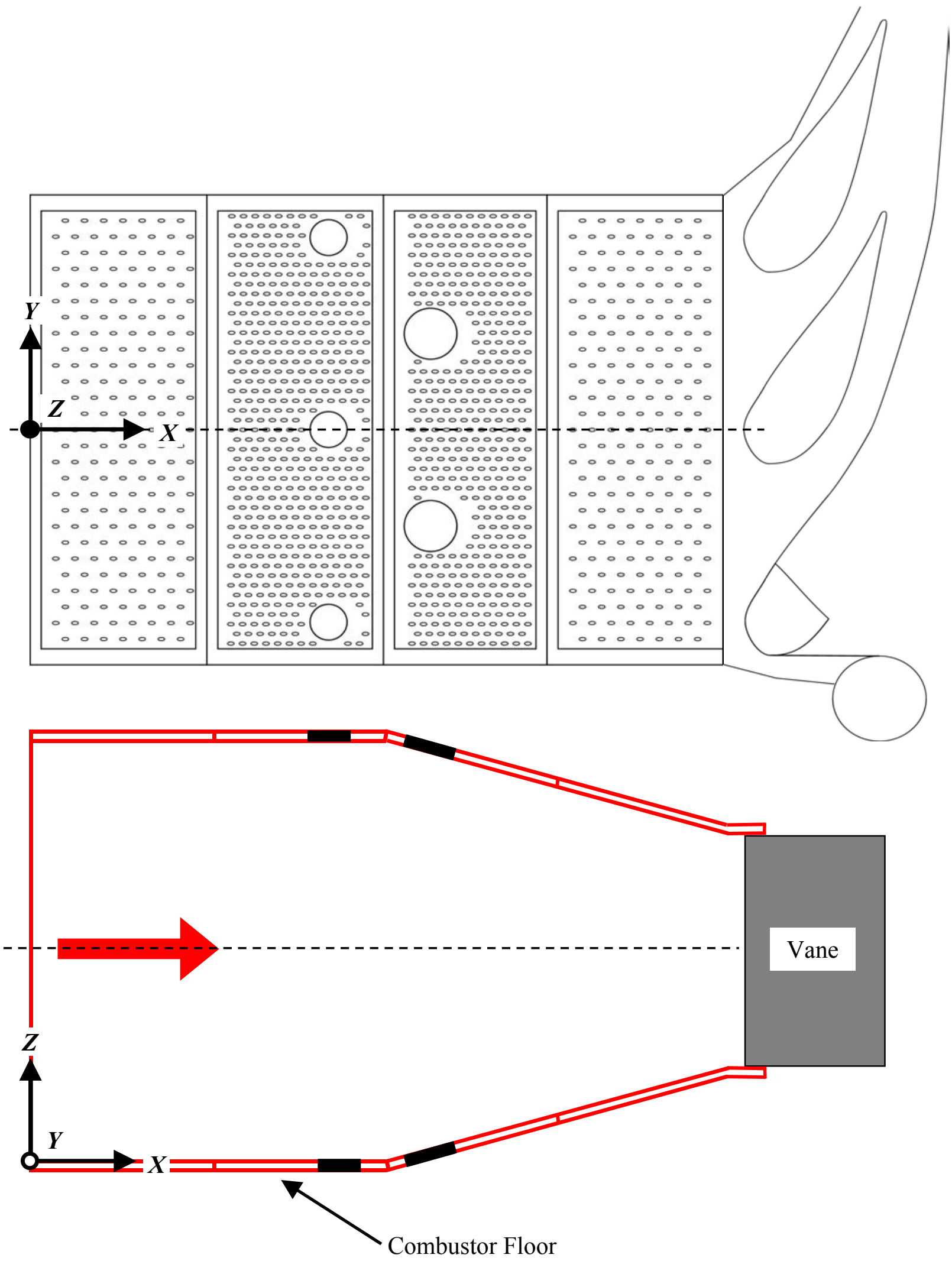

Figure 4.2 Global coordinate system within the combustor. 


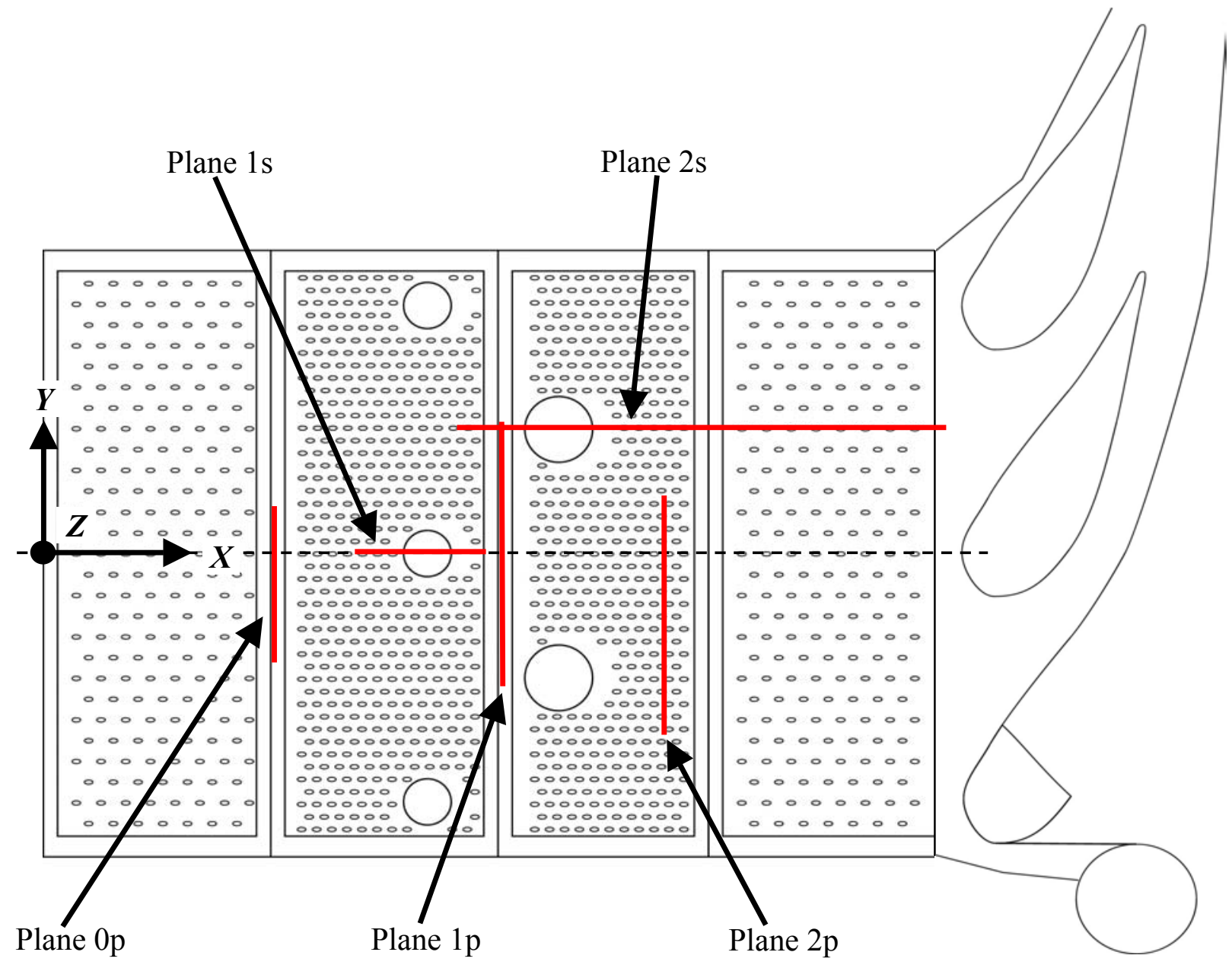

Figure 4.3 Location of the measured thermal field planes. 


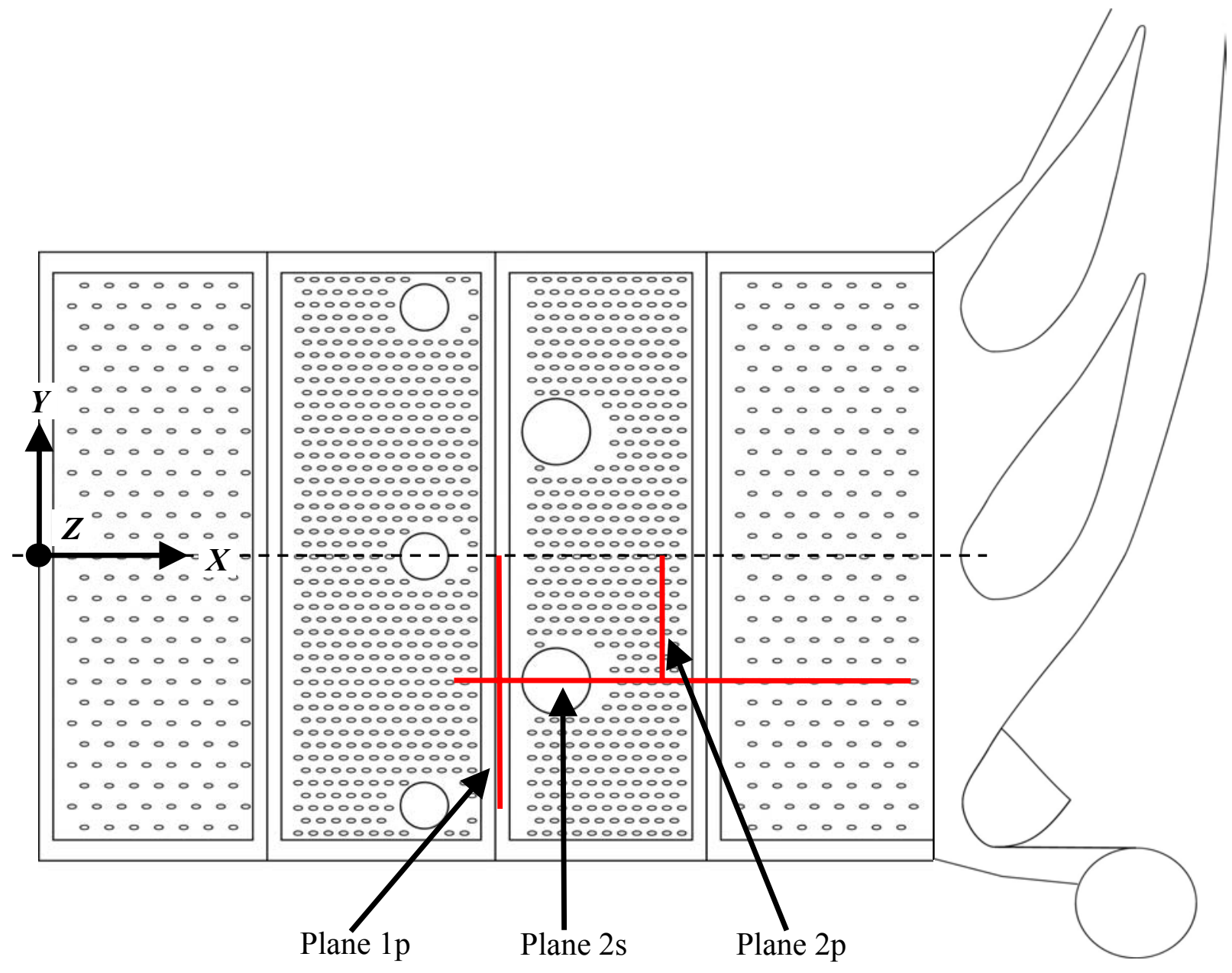

Figure 4.4 Location of the measured flow field planes. 


\section{Chapter 5: Thermal and Flow Field Results}

Having developed the proper test matrix, presented in Chapter 4, thermal and flow field measurements were performed to quantify and qualify the characteristics within the combustor. The first section of this chapter discusses various flow qualifications tests that were completed to verify flow periodicity and symmetry within the combustor simulator. The following section discusses the thermal field measurements performed for multiple rows of film-cooling, and addresses the effects behind varying the momentum flux ratio. The third section begins with detailed thermal and flow field measurements for high momentum flux dilution jets. Along with normalized temperature plots, the flow field results are presented in the form of secondary velocity vectors and contours of velocity, root-mean-square fluctuations, turbulent kinetic energy, turbulence intensity, and local turbulence intensity. The same thermal and flow field results are then presented for low momentum dilution jets, which are staggered and downstream of the high momentum jets. Recall that the sizes and locations of the thermal and velocity fields were described in Chapter 4 and can be seen in Figures 4.3 and 4.4, respectively. Finally, for both the high and low momentum dilution jets, thermal and flow field comparisons are made with a computational study previously completed by Stitzel (2001).

\subsection{Flow Qualification Tests}

Since much of the thermal and flow field data presented in this study was taken using the assumption that symmetry applied within the combustor simulator, it is important that this be demonstrated experimentally. This assumption was verified by assessing the results of four tests in particular. Figure 5.1 shows a vertical temperature profile taken at the trailing edge of plane 1 s and extending over approximately $83 \%$ of the total inlet height. One can see the downstream footprints of both dilution jets and, although this figure does not capture the film-cooling temperature profile at the top of the simulator, good symmetry about the mid-span is clearly visible. 
Measurements in plane $1 \mathrm{p}$ were taken directly downstream of the middle dilution hole in the first row of dilution jets. This particular hole lies centered directly at midpitch within the combustor simulator, and the thermal field results are shown in Figure 5.2. Below the thermal field contours is an indication of the measurement location, as in all of the figures within this chapter. As stated in Chapter 4, plane 1p extended approximately from $-0.27<Y / W<0.26$ in the pitchwise direction, therefore covering one-half of a sector (one vane pitch). Looking at Figure 5.2, as the dilution jet surges up, the center of the dilution jet is reasonably centered about mid-pitch $(Y / W=0)$. Note that there is slightly more jet spreading for $Y / W<0$ than for $Y / W>0$, which may be due to the jet interaction with the top row of jets. However, the kidney-shaped cooler region is centered about the mid-pitch. In all, these measurements indicate good symmetry across the pitch of the combustor simulator. Symmetry within the thermal fields implies symmetry within the velocity flow fields. In fact, the thermal fields are quite sensitive to variations in velocity. Obscurities in the thermal results serve as blatant indications to variations in velocity across the pitch or the span.

Figure 5.3 shows the experimental results for the three-component velocity measurements downstream of the first row of dilution (plane 1p). This particular plane spanned from the centerline of the tunnel and extended one full pitch towards the outer sidewall of the combustor simulator. The symmetry location was positioned at $Y / W=-$ 0.25. A dominant vortex, rotating in the counter-clockwise direction and downstream of the center dilution jet, is clearly illustrated within this figure. Also visible is another dominant vortex, rotating clockwise and belonging to the outer dilution jet that is centered about a $Y / W$ location of -0.5 . Having already demonstrated symmetry about the mid-pitch, these two vortices help to visually demonstrate periodicity between dilution jets within the combustor simulator.

In addition to periodicity, it is important that repeatability and the correct velocity transformations, particularly for the three-component measurements, are achieved. Agreement between different measurement planes serves as a test to verify that the measurement equipment is in proper working condition and is being used correctly by the experimenter. Figure 5.4 compares the profiles of the normalized streamwise, $u / u_{i n}$, and normalized pitchwise, $w / w_{i n}$, components of velocity at the intersection of planes $1 \mathrm{p}$ and 
2s. From Chapter 4, plane 1p was taken using a three-component LDV set-up, therefore allowing for all three components of the velocity to be deduced at each location. Plane $2 \mathrm{~s}$ was taken using only a two component LDV set-up, thus allowing for the determination of $u$ and $w$ only. Looking at the comparison of the intersection of planes $1 \mathrm{p}$ and $2 \mathrm{~s}$, excellent concurrence between both components of velocity is observed. Since both planes were taken weeks apart, repeatability within the simulator is proven. In addition to repeatability, Figure 5.4 validates the transformation equations used for in the threecomponent measurement planes.

\subsection{Film-Cooling Thermal Field Measurements}

Initial tests performed in this study were to document the thermal fields downstream of multiple rows of film-cooling jets. The objective was to determine the effect of tripling the momentum flux of the jets being injected from the combustor liner panels. The two cases studied were a momentum flux ratio, $I$, of 3 and 9 . Note that these momentum flux ratios are the average ratios for a given liner-panel. Recall from Chapter 4 that these momentum fluxes are set by measuring the difference between the plenum supply total pressure and the external static pressure. While the momentum flux ratios are tripled between the two cases tested, the mass flux ratio is only 1.8 times higher for the $I=9$ case than for the $I=3$ case. Thermal field measurements were made in two planes: plane $0 \mathrm{p}$, located immediately downstream of combustor liner panel one; and plane $1 \mathrm{p}$, downstream of dilution row one. For the $I=3$ case, $27 \%$ less mass flow was injected through the liner-panels than for the $I=9$ case. Also, the total-to-static pressures in the higher momentum flux case were 1.9 times higher than those for the lower momentum flux ratio.

Figures 5.5 and 5.6 show the results of the thermal field measurements made for plane $0 \mathrm{p}$. Recall that $\theta$ was defined as the difference in the freestream and the local measured temperature divided by the difference in the freestream and coolant temperature (equation 4.12). The measurement plane was located at $X / L=0.24$, which was two film-cooling hole diameters $(2 d)$ downstream of the trailing edge of the last row 
of cooling holes in panel one. Since the liner panels were made to represent a staggered film-cooling pattern, the dashed arrows have denoted the upstream film-cooling holes, whereas the solid arrows show the downstream holes. There were a total of 15 rows of staggered film-cooling holes present in panel 1 , with the hole spacing as indicated in Figure 3.4.

The jets closest to the measurement plane (the last row on panel 1) have their coolest region near the wall with a secondary cool region directly above. This secondary cool region is a remnant of the upstream film-cooling jet that exits from the aligned cooling hole. This effect is not noticed in the upstream holes (dashed arrows) because the two regions have mixed out by this location. Also at this location, it is apparent that the core of the jets from the upstream row is further away from the wall that the downstream row. As the jets progress downstream, the momentum flux of the jets results in a jet trajectory moving away from the wall.

A noticeable difference, however, between Figure 5.5 and 5.6 is the thickness of the film-cooling layers. For the $I=3$ case, the thickness of the film-cooling layer reaches a maximum of $Z / d=5.8$, which is $4.5 \%$ of the total combustor inlet height. For the $I=9$ case, the maximum $Z / d=7.4$, which is $5.7 \%$ of the inlet height. Clearly, this implies the penetration depth for the $I=9$ case is greater than for the $I=3$ case. However, the thicker film-cooling layer for the higher momentum flux does not automatically suggest that it is desirable. Although the film-cooling layer is thicker, the core of the jets is nominally at the same temperature level $(0.75<\theta<0.80)$ for both momentum fluxes. Furthermore, upon closer investigation, it looks as though the temperatures between the jets and near the wall are cooler for the $I=9$ case $(0.25<\theta<0.35)$ as opposed to the $I=$ 3 case $(0.15<\theta<0.25)$. The trade-off between using less coolant versus the slightly cooler near-wall temperatures is a decision to be made by the combustor designers. The results here suggest that by using less coolant, designers could divert the extra coolant to other areas within the combustor or turbine, perhaps improving the overall efficiency of the engine.

Lastly, Figures 5.7a and 5.7b show the effect of increasing the combustor liner momentum flux relative to the interaction with the downstream dilution jets. Note that while the film-cooling was significantly increased between these two cases, the dilution 
jet injection remained the same. Figure $5.7 \mathrm{~b}$ again shows slightly higher $\theta$ levels near the wall for the $I=9$ case relative to the $I=3$ case. However, no major cooling improvements are experienced along the liner wall. Just downstream of the dilution jet, the thermal field contours indicate that the film-cooling is being entrained by the upward motion of the dilution jet. Also the core of the kidney-shaped structure is slightly larger, has a higher penetration depth, and is slightly cooler ( $\theta=0.5$ higher $)$ at the core for the $I$ $=9$ case as opposed to the $I=3$ case.

\subsection{Thermal and Flow Field Measurements for High and Low Momentum Dilution Jets}

The primary function of a dilution jet within an aircraft gas turbine engine is to thoroughly mix with the combustor main flow to reduce $\mathrm{NO}_{\mathrm{X}}$ emissions and achieve high combustor efficiencies. It is desirable that these processes occur over the shortest combustor length possible, thereby decreasing the overall weight of the aero-engine. As one would expect, an accurate description of the temperature, velocity, and relative turbulence parameters is essential. As previously mentioned, this subsection will begin with the characterization of the high momentum dilution jets ( $I=128$ for the first row), which will then be followed up by the lower momentum dilution jets $(I=32$ for the second row).

\section{High Momentum Dilution Jets}

Figure 5.8 is a streamwise temperature profile through a first row dilution jet that was $8.51 \mathrm{~cm}$ in diameter $\left(D_{1}\right)$ and centered at $X / L=0.43$ and $Y / W=0$. The mass flux ratio, density ratio, and momentum flux ratio for this dilution jet were $12,1.12$, and 128 , respectively. Between $0.41<\mathrm{X} / \mathrm{L}<0.45$, one can see the coolest core of the dilution jet as it enters into the mainstream. The contours indicate that the jet reaches about $15 \%$ of the total combustor inlet height before the mainstream begins to bend its trajectory. The core of the jet penetrates up to $Z / H_{\text {in }}=0.3\left(3.5 D_{1}\right)$ before losing half of its thermal potential, and by the midspan $\theta$ has been reduced to 0.25 . An overall bulk reduction in 
the combustor's core temperature of $\theta=0.25$ was measured after passing through the first row of dilution.

Of particular interest is the film-cooling cooling interaction with the dilution jet itself. At the leading edge of the dilution jet, one sees a relatively thick layer of filmcoolant stagnating onto and being carried away by the dilution jet. Directly at the trailing edge, much of the film-coolant is entrained and carried away by the dilution jet, thus leaving behind a thinner film of coolant. This entrainment or recirculating region behind the dilution jet was also noted in Figure 5.7. When a dilution jet is injected into a crossflow, naturally a flow blockage is created and stagnant flow is left downstream of the jet. The shear forces at the trailing edge of the jet take advantage of the stagnate flow by entraining it, consequently creating what is known as the entrainment or recirculating region behind the dilution jet. Although no streamwise velocity measurements were made for this plane, the entrainment effects will quickly become apparent in the results of plane $2 \mathrm{~s}$, which was for a lower momentum dilution jet. In addition to these observations, temperature gradients at the jet-mainstream interface are quite large at the leading edge of the dilution jet. At the trailing edge of the jet, one can see these thermal contours spreading due to turbulent mixing induced by the dilution flow.

Although the top and bottom dilution jets were arranged inline with one another, the jets did not impact each other directly above their dilution hole centerlines. Instead, Figure 5.8 shows that the jets impact each other about $1.3 D_{1}$ downstream of the dilution jet center at $X / L=0.5$ (also refer to Figure 5.9). For clarity, the penetration depth of the dilution jet's core does not extend to the combustor midspan. The impact region at the midspan is a mixed out combination of the mainstream and film-coolant, transported up by the shear forces at the leading edge of the dilution jet, in addition to the jet itself.

Also shown in Figure 5.8 is the penetration and jet trajectory predicted by a correlation given by Lefebvre (1999). This correlation, for jets injected normal to the crossflow, is defined in equation 5.1:

$$
\frac{Z}{D}=0.82 \cdot I^{0.5} \cdot\left(\frac{X}{D}\right)^{0.33}
$$


where $D$ is the jet hole diameter. If the jet is injected into the crossflow at an angle $\theta$ less than $90^{\circ}$, the trajectory is obtained by merely multiplying $Z / D$ by $\sin (\theta)$. The solid line shows that the correlation over-predicts the trajectory of the jet and under-predicts the jet bending. A probable reason for these inaccurate predictions may be because of the inline, opposing dilution jets and that the momentum flux ratio for this particular jet is especially high $(I=128)$.

As discussed previously, Figure 5.7 shows the temperature profile nearly $8 d$ (6.2 $\mathrm{cm})$ downstream of the trailing edge of a first row dilution hole $(X / L=0.50)$.

Immediately noticeable is the large mushroom shaped temperature profile. This profile is the result of the counter rotating vortex pair described by Sgarzi and Leboeuf's (1997) model of jets in cross-flow. However, because this temperature profile is just downstream of two in-line, impacting jets, the counter-rotating vortex pair has spread further apart than would be expected for a single jet in crossflow. The centers of the mushroom shaped profile's two lobes are spaced at a $Y / W$ distance of 0.19 (38\% of a full pitch or 2 dilution hole diameters) apart. The overall width of the mushroom shaped structure is roughly $66 \%$ of one pitch or 3.5 dilution hole diameters, while there seems to be little interaction between the adjacent dilution holes, which are located at $Y / W=0.5$ and -0.5 .

It is important to note that the presence of ample film-cooling behind the dilution jet, even though there is only one row of film-cooling jets downstream the dilution hole. It is quite plausible that a horse-shoe vortex at the leading edge of the dilution flow has transported film-coolant from upstream to the trailing edge of the dilution jet (Sgarzi and Leboeuf, 1997). Furthermore, the entrainment of the film-cooling at the trailing edge of the dilution hole is clearly visible about the centerline of the dilution jet. However, these entrainment effects weaken with increasing distance from the core of the jet. This is confirmed as the non-mixed-out, cool cores of the numerous film-cooling jets become increasingly visible with growing distance from the jet centerline. On either side of the dilution jet, between $Z / H_{\text {in }}=0.07$ and 0.15 , no mixing of the hot combustor gasses with the dilution jet or the film-cooling is apparent, thus allowing a hot streak of gas to pass through the first row of dilution. 
Figure 5.9 shows the three-component velocity measurements for plane $1 \mathrm{p}$; in particular, contours of $u / u_{\text {in }}$ and cross-span vectors of $v / u_{\text {in }}$ and $w / u_{i n}$. As mentioned in Chapter 4 , all of the velocity quantities were normalized by the inlet velocity and the vectors, whose scale is given above the plots, were scaled to fit reasonably on the page. This measurement plane covered one entire pitch with one dilution jet centered at a $Y / W$ $=0$ and the other at $Y / W=-0.5$. However, the velocity data was concentrated about the first row, middle dilution jet $(Y / W=0)$.

The most immediate feature of Figure 5.9 is the counter-rotating vortex pair, seen in detail for the middle dilution jet, created as a result of the shear produced due to the dilution jet-mainstream interaction. This counter-rotating vortex pair was also illustrated by the model of Sgarzi and Leboeuf (1997). In addition, the centers of the counterrotating vortex pair seem to spread further apart, again due to the in-line, opposing rows of dilution jets impacting one another. The size of the counter-rotating vortex pair is quite large, extending over most of the vertical cross-sectional area within the measurement plane.

Near the center of the counter-rotating vortex exist the largest streamwise velocities with values as high as 3.25 times the inlet velocity (see Figure 5.9). Coincidentally, the contours of the streamwise velocity components are also mushroom shaped suggesting that the counter-rotating vortex formed by the jets travel downstream within the combustor simulator while dissipating into the mainstream. Along the centerlines of each dilution hole, particularly for the center dilution hole $(Y / W=0)$ between $Z / H_{\text {in }}=0.08$ and 0.24 , the streamwise velocity components are negative, which is indicative of the reverse flow in the recirculating region just downstream of the jet. These negative velocities are consistent with temperature planes $1 \mathrm{p}$ and $1 \mathrm{~s}$, where the film-cooling is entrained towards the dilution jet.

Near the dilution jet centerline, the opposite directions of the vectors slightly above and below midspan $\left(Z / H_{\text {in }}=0.5\right)$ confirm that the dilution jets, with the entrained film-coolant and mainstream flow, are impacting one another. The streamwise velocity magnitude is near zero at the midspan, suggesting that a stagnation area is created due to the impact region of the top and bottom dilution jets. 
The features of the outer dilution hole are somewhat distorted. This is clearly visible in the streamwise velocity contours where the mushroom shaped region lies lower and has a faster core than that of the middle dilution jet. In addition, the entrainment region behind the dilution hole is not as large. These discrepancies are believed to be a consequence of the combustor simulator walls, which are located at $Y / W=0.6$ and -0.6 . These walls on the combustor simulator are slightly constraining, for they did not allow for the outer dilution jets (located at $Y / W=0.5$ and -0.5 ) to fully develop in the pitchwise direction.

Figure 5.10 shows vectors of $v / u_{\text {in }}$ and $w / u_{\text {in }}$ over the temperature contours within plane $1 \mathrm{p}$. The flow and thermal fields are consistent with one another, showing the dominance of the counter-rotating vortex.

Figures 5.11a-c show contours of the streamwise, vertical, and spanwise rms levels for the velocity fluctuations for plane $1 \mathrm{p}$, which were all normalized by the inlet velocity. Within all three of the plots, the counter rotating vortex is clearly visible. In the streamwise direction (Figure 5.11a), the largest velocity fluctuations, $u_{r m s} / u_{i n}$, are found along the dilution jet centerline $(Y / W=0)$ at a $Z / H_{\text {in }}=0.28$. This location is where the core of the dilution jet begins to sharply bend over as it is swept away by the mainstream (see Figure 5.8). Values of the maximum streamwise fluctuations are as high as 1.9, while the lowest fluctuations can be found between the two dilution jets, at a $Y / W$ of 0.25 , ranging in value from 0.3 to 0.6 . Furthermore, since the midspan does correspond to the region of stagnation between the two in-line, impacting dilution jets (see Figure 5.9), extending as much as a $Y / W=0.15$ in either direction of the dilution jet centerlines $(Y / W=0$ and -0.5$)$, fairly large fluctuations ranging between 0.9 and 1.4 are found.

In Figure 5.11b, the largest pitchwise rms fluctuations are found slightly to the right of the center of the counter-rotating vortex, where the largest streamwise velocity components exist. Of the three fluctuations, the pitchwise fluctuations are the largest in magnitude reaching a maximum of $v_{r m s} / u_{i n}=3$. Note that these levels are quite different from the streamwise fluctuations indicating the highly anisotropic nature of the turbulence. Moreover, similar to the streamwise fluctuations, the lowest fluctuations are found between the two dilution jets while fairly large fluctuations exist at the midspan. 
In Figure $5.11 \mathrm{c}$, interestingly enough, the general shape still resembles that of a mushroom. However, unlike its two counterparts, the largest vertical fluctuations are found at the midspan over the dilution holes with values up to $w_{r m s} / u_{\text {in }}=2.5$. Since the top and bottom dilution jets are aligned, one would expect the largest fluctuations to be in the vertical component of the velocity at the area of impact.

Figures 5.12 and 5.13 are contour plots of the normalized combustor turbulent kinetic energy $\left(k / u_{i n}^{2}\right)$ and the turbulence levels $(T L)$, respectively, in plane 1p. Recall that the definitions for these quantities were given earlier in equations 4.15 and 4.17. Much like all of the rms fluctuation plots, the contours resemble the invariable mushroom shape. Furthermore, the highest turbulent kinetic energies and turbulence levels are yet again found slightly to the right of the center of the counter-rotating vortex, where the largest streamwise velocity components exist, in addition to the impact region between the two opposed dilution jets. For the turbulent kinetic energies, values as high as $k / u_{i n}^{2}$ $=6.8$ are found near the center of the counter-rotating vortex, and $\mathrm{k} / u_{\text {in }}^{2}=5.7$ within the impact region. Likewise, the turbulence levels are as high as $T L=2.1$ near the vortex center and $T L=2$ within the impact area.

Contours of the local turbulence $(T u)$, which was defined by equation 4.19 , are shown in Figure 5.14. Since the turbulent kinetic energy and turbulence levels were nondimensionalized according to the inlet velocity, it is difficult to determine the most turbulent regions with relation to the local velocity. The local turbulence level is normalized point by point with respect to the local velocity magnitude. This contour provides the exact location of the most turbulent region within each measurement plane. For example, in an extremely high velocity region, the local turbulence level may seem high with respect to the local mass averaged velocity; however, when calculated with respect to the local velocity, the turbulence level will be significantly less. According to Figure 5.14, the highest local turbulence levels are found at the midspan, on the dilution jet centerline where the impact region dominates and the velocities are the lowest. In general, turbulence levels are still quite high at an average of $24 \%$ over most of the plane. 


\section{Low Momentum Dilution Jets}

While the first row of dilution jets was directed vertically, the second row was angled at $15.8^{\circ}$ from the vertical axis. The dilution jets were injected near the start of the contraction section in the combustor simulator, and located $0.90 \mathrm{~m}$ downstream of the start of the combustor simulator at $X / L=0.57$. The second row of dilution holes were approximately 1.4 times larger in diameter than the first, at $12.1 \mathrm{~cm}$, and their centerlines were staggered with respect to those of the first row. The second row contained two dilution jets on the top and bottom, and like the first row. the top and bottom rows were aligned with one another in the pitchwise and streamwise directions. The momentum flux for the second row of dilution jets was significantly smaller than the first at $I=32$, while the mass flux and density ratios were 6 and 1.12, respectively.

Figure 5.15 illustrates contours of temperature in a streamwise plane through the centerline of the inner dilution hole $(Y / W=0.25)$ within the second row. Plane $2 \mathrm{~s}$ begins slightly before the second row of dilution and extends through the end of the combustor simulator. The primary purpose of the second row of dilution is to complete combustion before the flow enters the first stage turbine vanes. For this reason, the momentum flux of the second row was reduced to $I=32$ while nearly maintaining the same mass flow addition across the entire row ( $8.8 \%$ of the exit mass flow) as for the first row of dilution.

The core of the dilution jet indicates a change in trajectory from initially towards the upstream and then switching towards the downstream. When comparing the respective penetration heights of the $\theta=0.90$ temperature contour for the first and second rows of dilution, a large discrepancy is not readily apparent. Looking at the $0.9<\theta<$ 0.95 temperature contour, the first row penetrated a $Z / H_{\text {in }}=0.10$ while the second row, relative to the injection location, penetrated a total $\Delta Z / H_{\text {in }}=0.13$. On the other hand, when relating this height to the respective dilution hole diameters, opposite results are noted. The first row penetrated to a $Z / D_{l}=1.2$, whereas the second row only penetrated to a $Z / D_{2}=1.1$.

Interestingly, at the beginning of plane $2 \mathrm{~s}$, the freestream temperature is the same as the inlet temperature. The spreading of the jets from the first row of dilution is not apparent until a $X / L=0.55$, where the counter-rotating vortices of the first row of dilution jets have had a chance to expand and mix out the flow in the pitchwise direction. At $X / L$ 
$=0.55$ and along the midspan $\left(Z / H_{\text {in }}=0.5\right)$, the combustor core "bulge" in temperature is due to the first row of dilution.

The trailing edge of the dilution jet injection hole was located at $X / L=0.61$, while the next film-cooling injection location was not until approximately $8 d$ downstream at $X / L=0.65$. The presence of film-cooling at the trailing edge of the dilution hole in Figure 5.15 is again indicative of a possible horse-shoe vortex at the wrapping around the leading edge of the dilution jet. Recall that this phenomenon was also seen in the pitchwise thermal field behind the first row of dilution jets (Figure 5.7).

As mentioned earlier, the temperature results at the trailing edge of the first row dilution jet (plane 1s) indicated a recirculating region where much of the film-cooling was entrained and carried away by the dilution jet leaving behind a thinner film of coolant. This phenomenon is also evident in the thermal results of plane $2 \mathrm{~s}$. At an $\mathrm{X} / \mathrm{L}=$ 0.65 , a thin layer of film-coolant is visible, while downstream of the second dilution row, the film thickness grows substantially due to the high turbulence levels (shown later in this section) produced by the dilution jets.

Lastly, the solid line on Figure 5.15 indicates the dilution jet trajectory predicted by Lefebvre's correlation (1999). Like the higher momentum first row of dilution jets, the correlation predicts the trajectory of the flow fairly precisely near the liner panel, but underpredicts the bending of the jet as it enters into the mainstream. Again, this missprediction may be due to the effects of the opposing jets. Furthermore, although this correlation applied to jets injected at various angles and not only to jets injected perpendicularly to a crossflow, the solid line shows no indication of the shift in trajectory from towards the upstream, near the wall, to the downstream, at $Z / H_{i n}>0.18$. It is worth noting, however, that overall this correlation appears to fit better for this lower momentum dilution jet as opposed to the higher momentum jets (Figure 5.8).

Figure 5.16 shows contours of temperature in plane $2 p$, which was located one dilution hole diameter $\left(1 D_{2}\right)$ downstream of the trailing edge of a dilution two hole. This plane almost covers one full pitch and shows both the first and second rows of dilution in reasonable detail. On the right, the middle first row of dilution hole, which is denoted by the dashed lines and arrow, is clearly visible. At this point in the combustor, the mushroom shaped temperature profiles have mixed together, and a warmer region near 
the midspan has emerged. It is likely that this warm region results from the unmixed warmer fluid flowing around the stagnation region, which was created by the impact of the opposing dilution jets in the first row. The warmer region $(0.25<\theta<0.3)$ seen along $Y / W=0$ and a height of $Z / H_{\text {in }}=0.15$ is the footprint of the warm fluid trapped in the recirculating region downstream of the first row of dilution jets.

On the left of Figure 5.16, the thermal contours of the second row of dilution (depicted by the solid lines and arrow) are visible. The second row dilution jet has turned at this point, and is being swept down the remaining length of the combustor (see Figure 5.15 also). Overwhelmingly apparent, however, is the lack of uniformity within the combustor exiting profile at this point. At the midspan, the remnants of trapped fluid behind the first row dilution jet show core temperatures ranging between $0.3<\theta<0.35$. The second row dilution jet's core is almost twice as cold with values between $0.55<\theta<$ 0.6 located $Y / W=-0.25$ and $Z / H_{\text {in }}=0.3$. With only $31 \%$ of the combustor's length left and no more rows of dilution, a streaky, non-uniform temperature profile at the combustor exit is highly likely. A non-uniform temperature field, which implies a lack of proper mixing, is clearly undesirable for a number of reasons including burnouts on the turning vanes and unburned fuel exiting the combustor.

Vectors of $u / u_{\text {in }}$ and $w / u_{i n}$, taken in the streamwise direction along the centerline of the outer second row dilution hole $(Y / W=-0.25)$, are shown in Figure 5.17. Consistent with the thermal field results (Figure 5.15), between $0.54<X / L<0.60$, the ejection of flow through the dilution hole is obvious. Beginning at $Z / H_{\text {in }}=0.3$, the core of the dilution jet is deflected sharply by the combustor mainstream. Most notable, however, is what happens at the leading and trailing edges of the dilution hole. At the leading edge, the flow is angled downward as it impacts the dilution jet. The most probable explanation for this behavior is that the flow is being deflected under the stagnation region created at the midspan by the first row of dilution jets. Furthermore, as the flow stagnates onto the dilution flow, it is quickly deflected up by the jet. Downstream of the jet ejection, the vectors clearly illustrate the separated, recirculating region behind the dilution jet where some of the film-coolant flow is entrained by the shear forces created by the dilution jet. 
Downstream of the dilution hole, the flow is continuously accelerated along the combustor surface due to the contraction. Specifically, the flow field vectors show an acceleration in the near wall region, due to added film-cooling flow, and above the jet injection at the mid-span, due to flow bypassing the dilution jet blockage. In general, as the flow is accelerated, the velocity profiles become more uniform towards the combustor exit. However, traces of the faster film-cooling and midspan regions are still visible in the streamwise velocity profiles at the combustor exit.

Lastly, Figure 5.18 shows the measured flow field superimposed on the thermal field in plane $2 \mathrm{~s}$. The velocity vectors out of the dilution hole, between $0.55<X / L<$ 0.60 , follow the trajectory of the dilution jet core, shown by the temperature contours, rather precisely. Most notable, however, is the entrainment of the film-cooling at the trailing edge of the dilution jet. The vectors show the effect of the shear forces at the trailing edge of the dilution jet. As the flow is entrained into the jet, the temperature contours immediately behind the jet show a thinner layer of film-cooling. As the entrainment effects weaken further away from the jet, the flow reverses and the vectors seem to follow along the temperature contours, which are growing thicker due to the enhanced turbulence behind the dilution jet.

Normalized contours of the $u_{r m s}$ and $w_{r m s}$ fluctuations can be seen in Figure 5.19. In the streamwise component of velocity, the largest fluctuations are centered at $X / L=$ 0.61 and $Z / H_{\text {in }}=0.25$. This location corresponds directly to the flow reversal region at the trailing edge of the dilution jet, where the entrainment effects are overcome by the bending of the dilution jet as it is swept away by the mainstream. The largest fluctuations in the vertical component of velocity $\left(w_{r m s}\right)$ occur along the leading edge of the dilution jet where a strong shear layer is present due to the jet-mainstream interaction. In addition, a high fluctuation region is also apparent at the trailing edge of the jet, near the combustor surface $\left(Z / H_{\text {in }}=0.1\right)$, where the dilution jet begins to entrain much of the filmcooling flow. Lastly, large fluctuations exist for both velocity components along the liner wall where the film-cooling flow is ejected, while in the mainstream the fluctuations decay as the flow is accelerated through the combustor test section. 
Figures 5.20 and 5.21 show contours of the normalized combustor turbulent kinetic energy $\left(\mathrm{k} / \mathrm{u}_{\text {in }}^{2}\right)$ and the turbulence levels $(T L)$, respectively, for plane 2s. The definitions for these two quantities were given previously in Chapter 4 by equations 4.16 and 4.18. In the case of plane $2 \mathrm{~s}$, large values of the combustor turbulent kinetic energy and turbulence levels are found throughout the dilution jet, especially at the leading and trailing edges, and at the film-cooling hole exits. Recall that at the leading and trailing edges of the jet, the largest shear layers exist due to the interaction of the jet with the mainstream and the large recirculation region. Figure 5.22 shows the local turbulence levels $(T u)$ and indicates the largest levels in two primary regions. The first region is near the trailing edge of the dilution jet $(X / L=0.63)$ in the recirculation zone. The second region is located at $X / L=0.68$ and $Z / H_{\text {in }}=0.27$, where the entrainment effects vanish, the flow redirects itself towards the downstream, and the dilution jet does not further penetrate vertically into the flow. The average local turbulence level near the combustor simulator exit $(X / L=0.92)$ was found to be $24 \%$. Barringer (2001) also made turbulence measurements at the exit of the combustor simulator used in this study. His measurements, also along the centerline of a second row dilution hole, indicated nearly the same local turbulence levels of approximately $18 \%$ at the midspan, as opposed to the $22 \%$ found at the midspan by these experiments.

A study by Pietrzyk (1989) noted similarly high regions of turbulence around film-cooling jets in crossflow. The inclined film-cooling jets were injected into a crossflow at a $35^{\circ}$ angle. For the case considered here, the density ratio and the jet-tofreestream velocity ratio were both unity. In all, the geometry and velocity ratio of Pietrzyk's jets varied considerably from the dilution jet studied in plane 2s. For this reason the large recirculation zone behind the dilution jet was not experimentally captured behind the film-cooling jets. Instead a wake region behind the trailing edge of the jet was noted, and the interaction of the shear layer over the wake region was similar to the shear layer interactions seen atop the recirculating region noted in this study. Specifically, Pietrzyk noted high levels of turbulence (18\%) generated at the leading edge of the jet-to-mainstream interaction. He found that these levels of turbulence dissipated quickly downstream of the trailing edge of the hole. He also noted the largest levels of 
turbulence $(20 \%)$ in the shear layer over the wake region in the lee of the jet. Two filmcooling hole diameters downstream of this region, the vertical rms level was found to be slightly higher (by $2 \%$ ) than the streamwise rms levels. The regions of high turbulence found by Pietrzyk are consistent with those noted in this study for a low momentum dilution jet.

Three component velocity measurements are presented for plane $2 p$ in Figure 5.23. This plane was taken one dilution hole diameter $\left(1 D_{2}\right)$ downstream of the trailing edge of the outer dilution hole in the second row $(\mathrm{X} / \mathrm{L}=0.69)$. The flow field plane is slightly smaller than the thermal plane, extending from the centerline of middle dilution hole in row one to the centerline of the outer dilution hole in row two $(-0.25<Y / W<0)$. Surprisingly, these results hardly resemble those of plane $1 \mathrm{p}$, primarily because the strong kidney vortex seen behind the first row of dilution is not evident at this location. Slight traces of it can be seen near the combustor mid-span, however it is most likely that the high turbulence levels, as will be illustrated in the upcoming figures, have caused considerable reductions in the swirl velocities. The $v / u_{\text {in }}$ and $w / u_{\text {in }}$ vectors indicate a strong upward flow near the combustor wall due to the contraction within the combustor. Furthermore, the streamwise contours of $u / u_{\text {in }}$ show that the flow has accelerated to 3 times the inlet velocity near the midspan, and 4 times the inlet along the liner panels due to the film-cooling ejection.

One major characteristic that is the same in Figure 5.23 as in Figure 5.9 is that the three-component flow field just behind the first row of dilution jets, is the recirculating region behind the dilution jet. At $Z / H_{\text {in }}=0.25$, slightly negative streamwise velocities are visible, while along the film-cooling panel the $v / u_{\text {in }}$ and $w / u_{\text {in }}$ vectors exhibit a leftward sweeping motion. Both of these characteristics are indicative of the entrainment behind the dilution jet. Furthermore, the non-uniformity in the streamwise velocity component asserts the non-uniform combustor exit profile, which was clearly apparent in the temperature contours for plane $2 p$ (Figure 5.16). The streamwise velocity outside of the dilution jet affected region (along $Y / W=0$ ), varies as much as $\Delta \mathrm{u} / \mathrm{u}_{\mathrm{in}}=0.5$ from a $Z / H_{\text {in }}=$ 0.2 to midspan. Lastly, Figure 5.24 shows the $v / u_{\text {in }}$ and $w / u_{\text {in }}$ velocity vectors superimposed on top of the thermal field contours in plane $2 \mathrm{p}$. The sweeping of the coolant toward the second row dilution jet as well as the acceleration near the combustor 
wall is visible. However, the vectors show little indication, such as dominant vortical structures, that may aid in further mixing out the non-uniform temperature field.

Comparatively, the rms-fluctuations within this plane, although still high, are relatively low in comparison to plane $1 \mathrm{p}$ due to the acceleration of the flow by the contraction. Figures 5.25a-c show the $u_{r m s} / u_{i n}, v_{r m s} / u_{i n}$, and $w_{r m s} / u_{i n}$ contours for plane $2 \mathrm{p}$ and illustrate the anisotropic nature of the turbulence created by dilution jets. The largest fluctuations in the streamwise velocity (Figure 5.25a) are found near a $Z / H_{\text {in }}=0.3$. This peaked region is located slightly above where the direction of the velocity vectors changes within the flow reversal region. It is also where the jet's trailing edge mixes into the mainstream and the streamwise jet velocity component has the same magnitude as the mainstream. In Figure 5.25b, the largest $v_{r m s} / u_{\text {in }}$ levels are again found within the recirculating region, particularly near the combustor surface $\left(Z / H_{\text {in }}=0.12\right)$ and the top of the region where the flow is changing direction $\left(Z / H_{i n}=0.35\right)$. Lastly, the $w_{r m s} / u_{\text {in }}$ levels are the lowest of the three (see Figure 5.25c). Naturally, the highest values are found along the dilution hole centerline atop the recirculating region $\left(Z / H_{i n}>0.3\right)$. However, these fluctuations are the lowest due in part to the fact that the $w$-component of velocity is extremely small, with the exception being near the exit of the film-cooling holes.

Contours of the normalized combustor turbulent kinetic energy $\left(k / u_{i n}^{2}\right)$ and the turbulence levels (TL) are shown in Figure 5.26 and 5.27, respectively. The largest values of these quantities are found near the combustor surface at a $Z / H_{\text {in }}=0.12$, and above the recirculating region at a $Z / H_{\text {in }}=0.32$. As previously mentioned, the shear forces in these turning regions produce the highest turbulence within the flow. Figure 5.28 shows that the local turbulence levels $(T u)$ are the highest at the top of the recirculating region $\left(Z / H_{\text {in }}=0.26\right)$ where the largest velocity gradients and smallest velocity magnitudes are present. The turbulence levels, however, in relation to the rest of the flow field are not as large because of the high streamwise and vertical velocity components near the combustor simulator exit. 


\subsection{Comparisons Between Experimental and Computational Results}

To compare measured results with computational predictions, temperature and flow field data was extracted from a computational study completed by Stitzel (2000). This data was extracted for planes $1 \mathrm{p}, 2 \mathrm{~s}$, and $2 \mathrm{p}$. Table 5.1 is a comparison between the mass-flow conditions studied here and those she simulated computationally. Although these conditions were not exactly the same, they are well within reason to provide a more than adequate and practical comparison.

Figure 5.29 is a comparison of normalized temperature contours in plane $1 \mathrm{p}$. Both plots seem to exhibit the same characteristics; however, there are two major differences. The half-mushroom shaped temperature contour in the computational results is much thinner and more reminiscent of a streak rather than a cloud, while the entrainment of the film-cooling in the recirculating region is not as evident.

Turbulence modeling is a necessity resulting from a closure problem in the averaged continuity and transport equations. Therefore, inherently exact solutions to turbulent flows are not available. Secondly, recent advancements in turbulence modeling, such as large eddy simulations, require incredibly powerful computers to provide even more accurate solutions. Often, the trade off between time and accuracy is minimal in its overall benefit. The model used for this computational study was the RNG $K-\varepsilon$ model. This model is a statistical model derived using renormalization group theory, and includes an additional term to the dissipation equation that accounts for rapid strain and streamline curvature effects. Although this model is relatively robust and provides reasonable accuracy while being computationally economical, it is still not completely precise as shown in Figure 5.29. This particular model especially lacks accuracy in complex flows were streamline curvature, swirl rotation, and rapid changes in strain rates are present; i.e. the impact of two in-line dilution jets. As a result, computations tend to incorrectly predict mixing, thereby leading to an underprediction in the turbulence. This effect is clearly present in the computational temperature results where one can see a spike of cold fluid rather than a mixed-out mushroom-shaped cloud (Figure 5.29).

Figure 5.30 is a comparison of the velocity vectors and contours predicted computationally and measured experimentally in plane 1p. Again, the counter-rotating 
vortex and recirculating region directly behind the jet are present. For the computational case, however, the contour-rotating vortex is much stronger, as evidenced by the larger $v / u_{\text {in }}$ and $w / u_{\text {in }}$ vectors especially near the midspan. The recirculating region is larger in size and lies higher than for the experimental case. Lastly, at the midspan and along the dilution jet centerline $(Y / W=0)$, the streamwise velocity is approximately three times the inlet velocity. For the experimental results, the streamwise velocity magnitude in the same region is nearly zero, indicative of a stagnation region. In all, these discrepancies indicate that the computational results underpredict the mixing of the two dilution jets and overpredict the trajectory and velocity of the streamlines exiting the dilution hole.

Experimental and computational thermal results for plane $2 \mathrm{~s}$ are given in Figures 5.31a and 5.31b. As stated in the previous section, due to the RNG $K$ - $\varepsilon$ model's underprediction of the turbulence, the computational results often appear much less diffuse than in the experiments. This is clearly evident in Figure $5.31 \mathrm{~b}$ where the core of the computational dilution jet is much more concentrated and penetrates much further than the experimental results. High thermal gradients are seen at the leading edge of the dilution jet in both figures. However, the entrainment of film-coolant directly downstream of the dilution jet is fairly weak in the computational results while the thickness of the film-cooling behind the dilution jet is much greater for the experimental results than for the computational predictions. This comparison is consistent with the large underprediction of turbulence by the computational model.

Figures 5.32a and 5.32b show a comparison of the flow field vectors for plane 2s. The entrainment behind the dilution jet and the acceleration of the flow through the combustor are present in both plots. The penetration of the dilution jet is much higher for the computational results as compared with the experimental results. Also present is the downward flow at the leading edge of the dilution jet. This downward flow, however, appears to be overpredicted in the computational results. Recall that the experimental results showed the same downward motion, which is most probably the result of the mainstream flow being deflected under the stagnation region at the midspan of the first row dilution jets. The underprediction of the mixing at the impact of the first row dilution jets is attributed to the overprediction of the downward velocities as compared with the experimental results. Figure 5.33 shows streamlines exiting the first row of 
dilution holes. The predicted impact of the jets causes the streamlines turn a full 180 degrees. The streamlines exiting the dilution hole initially move towards the upstream flow direction (at the midspan) and then towards the downstream as the flow is swept away by the mainstream. Following the streamlines downstream indicates that the jet flow is being directed away from the dilution jet centerline. Furthermore, Figure 5.33 indicates that the dilution jets are stronger and more coherent, thus a larger recirculation zone is predicted. This larger zone in turn indicates an underpredication in mixing at the impact region of the first row dilution jets. In all, the larger recirculation zone and the mainstream flow sweeping under this flow blockage are therefore exaggerated at the leading edge of the jet. The size of the impact region is overpredicted when compared with experiments.

Of all the experimental and computational comparisons, the largest discrepancies exist in plane 2p. Figure 5.34 shows thermal field comparisons for plane 2p. Quickly apparent is the large non-uniformity of the computational thermal field in comparison to the experimental. The major features are still apparent, particularly the entrainment behind the dilution jets. It is interesting, however, that the computationally predicted thermal field behind dilution row two slightly resembles a mushroom shaped profile suggesting the presence of a counter-rotating vortex.

Figure 5.35 shows a comparison of $u / u_{\text {in }}$ contours and $v / u_{\text {in }}$ and $w / u_{\text {in }}$ vectors measured experimentally with those calculated computationally. For the streamwise contours, the general characteristics remain; that is, the entrainment of the flow behind the dilution jet is dominant. In the velocity vectors, however, the large discrepancy becomes obvious. The computational results by Stitzel (2001) show the flow near the surface being entrained by the dilution jet injection. As a result, much of the flow is clearly drawn to the left and is consistent with the experimental observations. However, it is the three primary vortices shown by the computational results that point out a clear difference. These vortices are most probably the remnants of the first row of dilution jets interacting with the mainstream and second row of dilution jets. As stated before, the CFD has grossly underpredicted the turbulence, thus allowing the vortical structures to remain coherent up to this location. This becomes very evident near the combustor exit where, in reality, many of the vortices visible in the computations have been diffused. 
Although for the most part the CFD predictions show similar behaviors, the need for experimental verification is proven evident by the comparisons made in this section. 
Table 5.1 Comparison Between the Experimental and Computational Conditions for the Combustor Simulator

\begin{tabular}{|l|c|c|}
\hline & $\begin{array}{c}\text { \% of Exit Mass Flow } \\
\text { (Experimental) }\end{array}$ & $\begin{array}{c}\text { \% of Exit Mass Flow } \\
\text { (Computational) }\end{array}$ \\
\hline Panel 1 & 1.19 & 1.50 \\
\hline Panel 2 & 2.96 & 3.50 \\
\hline Dilution Row 1 & 8.73 & 8.75 \\
\hline Panel 3 & 3.82 & 3.50 \\
\hline Dilution Row 2 & 8.82 & 8.75 \\
\hline Panel 4 & 2.05 & 1.50 \\
\hline
\end{tabular}




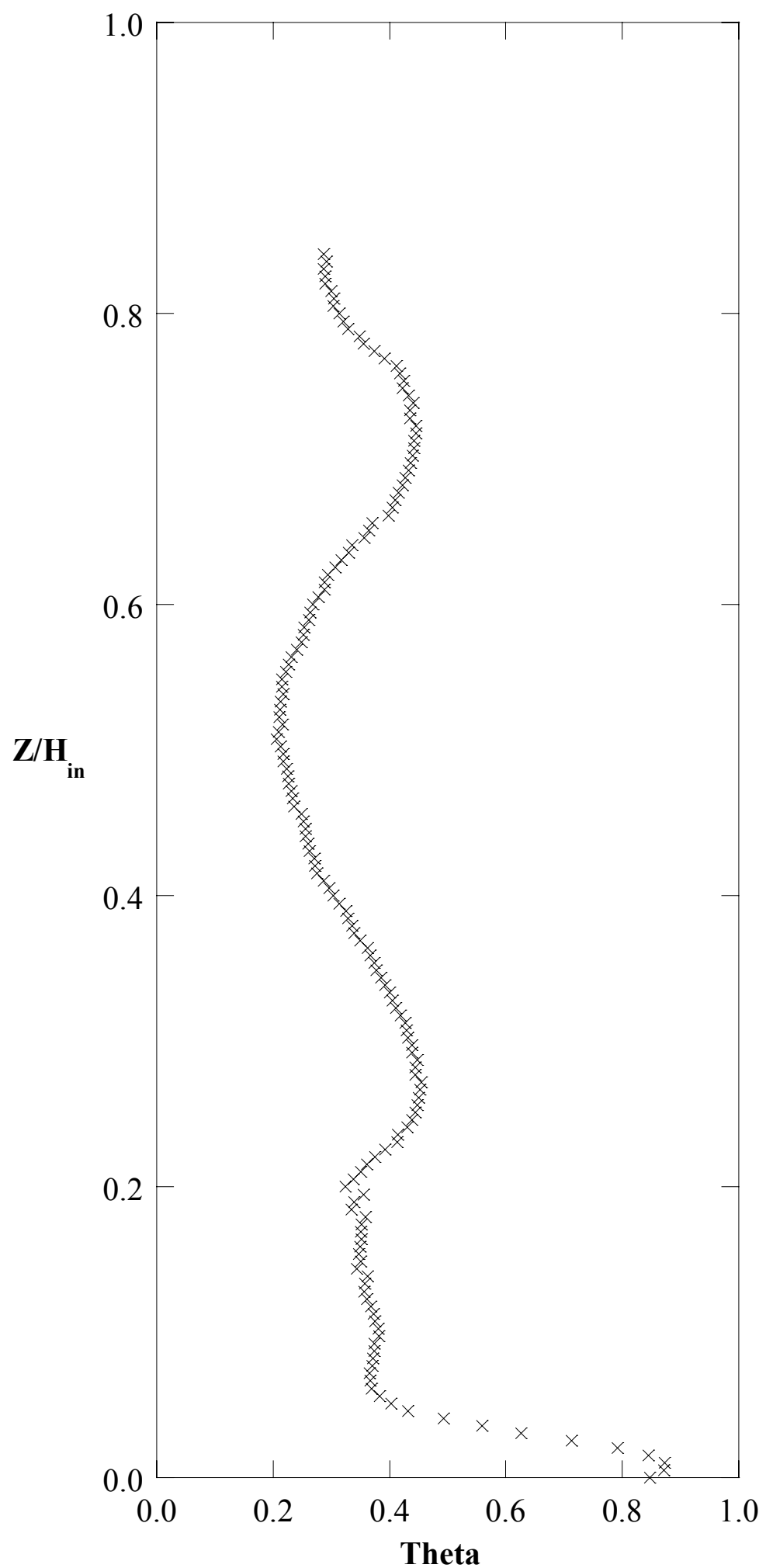

Figure 5.1 Temperature profile showing symmetry at the trailing edge of plane $1 \mathrm{~s}$ $(X / L=0.50)$. 


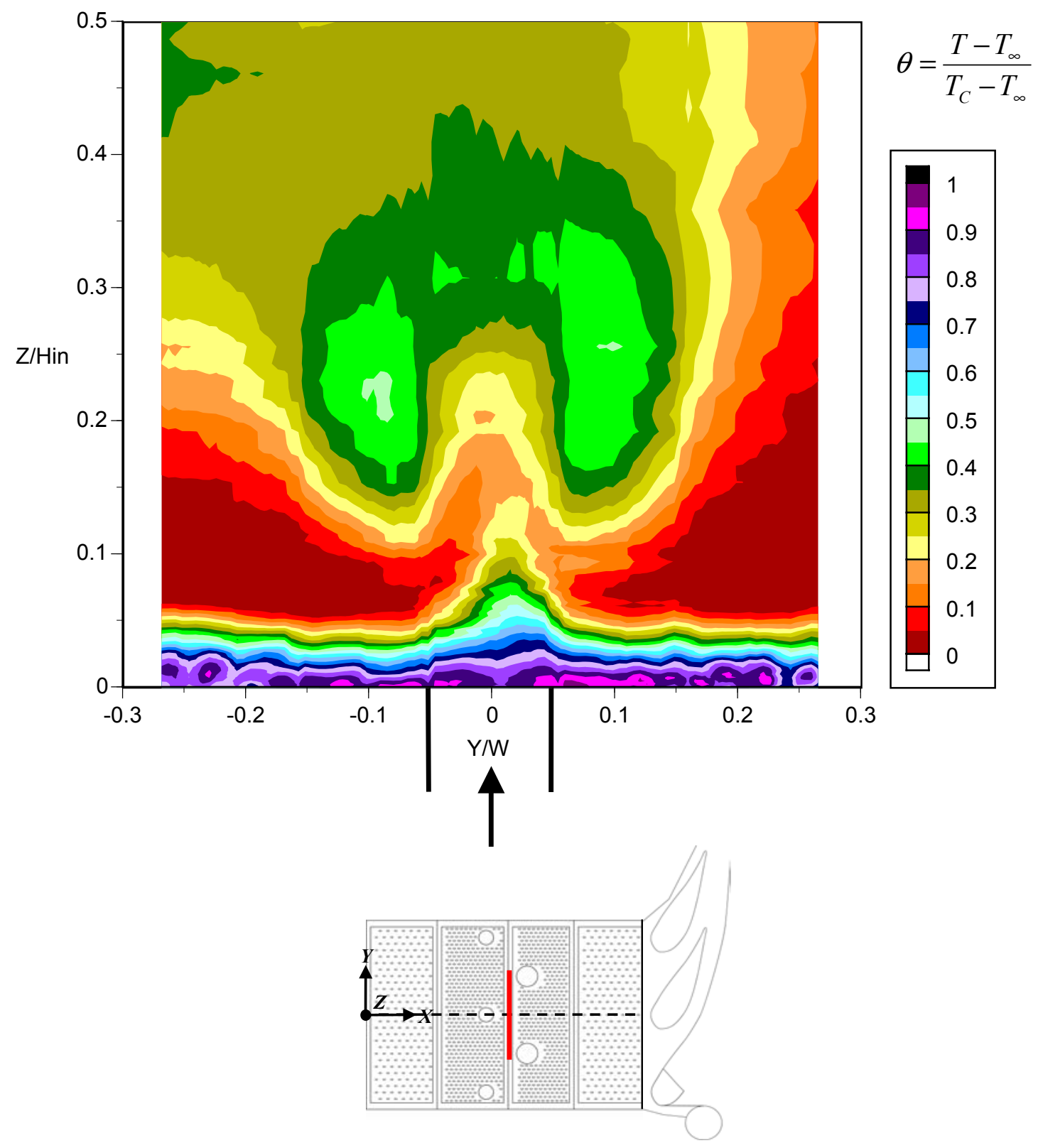

Figure 5.2 Temperature contours showing symmetry in the pitchwise direction for plane $1 \mathrm{p}$. 


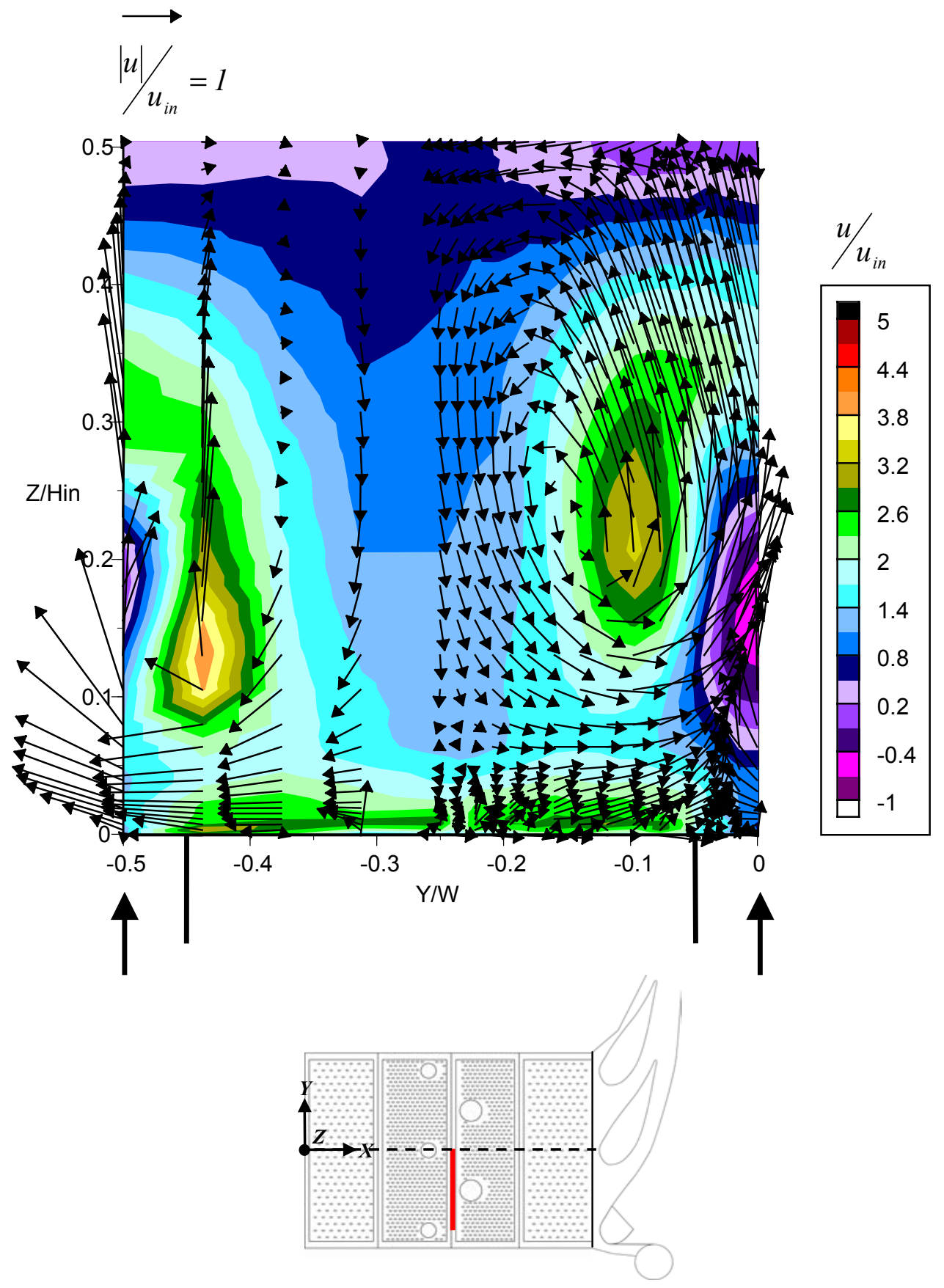

Figure 5.3 Velocity measurements demonstrating symmetry and periodicity between dilution jets in the pitchwise direction. Profile was taken for plane 1s. 

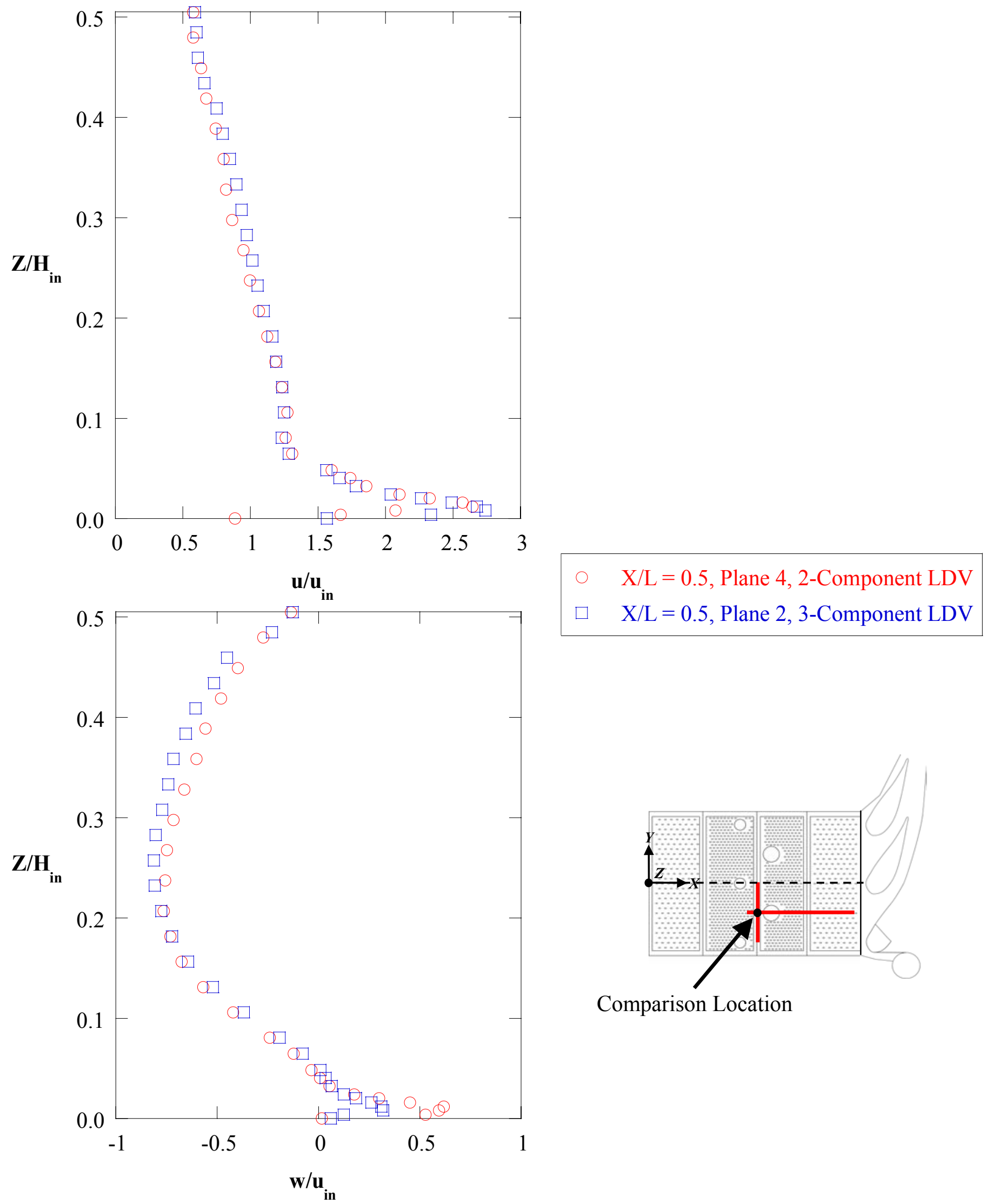

Comparison Location

Figure 5.4 Proof of repeatability and velocity transformations for planes $1 \mathrm{p}$ and $2 \mathrm{~s}$. 


$$
\theta=\frac{T-T_{\infty}}{T_{C}-T_{\infty}}
$$
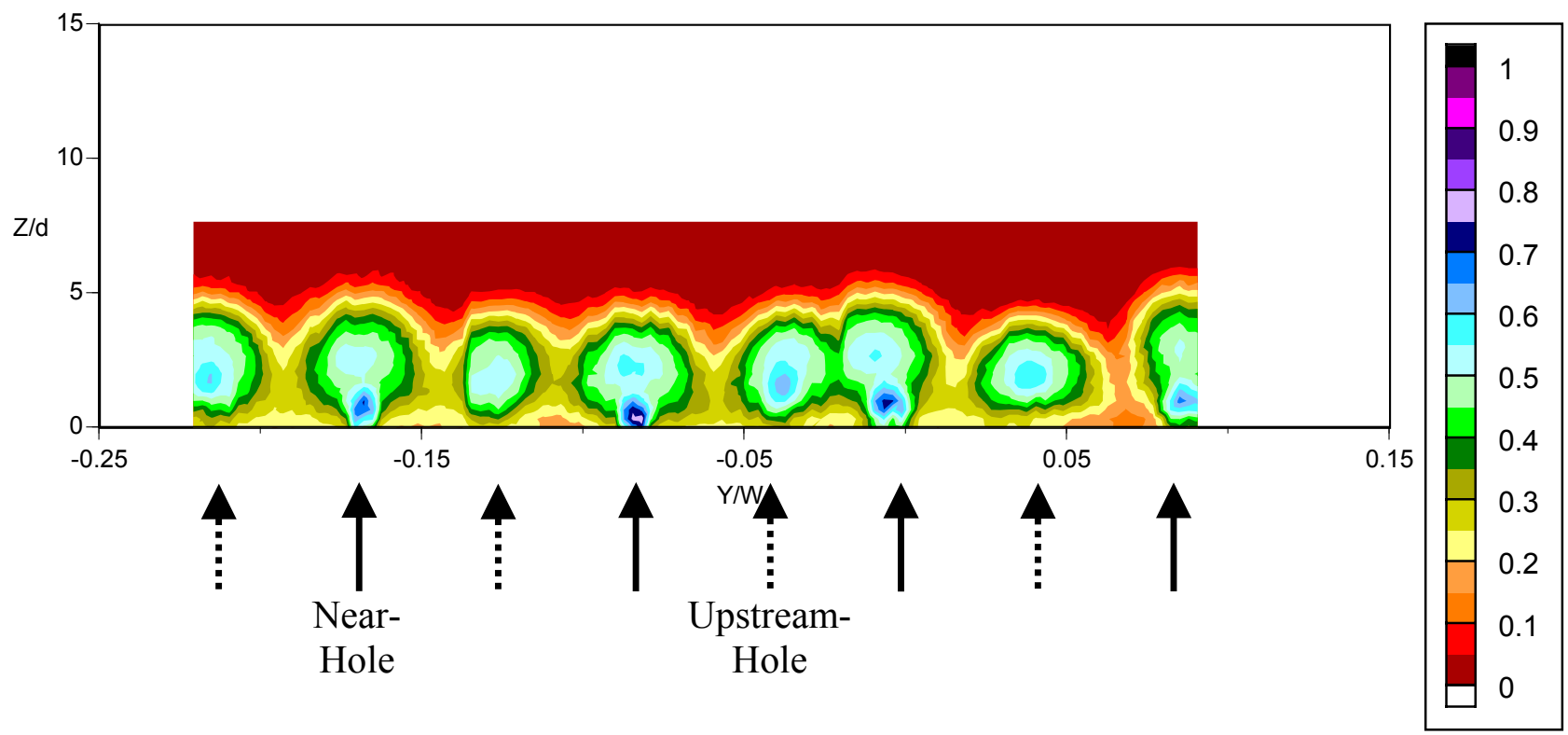

Figure 5.5 Film-cooling thermal field measurements at $X / L=0.24$ in plane $0 \mathrm{p}$ for $I=3, M=1.8$ and $D R=1.08$.

$$
\theta=\frac{T-T_{\infty}}{T_{C}-T_{\infty}}
$$
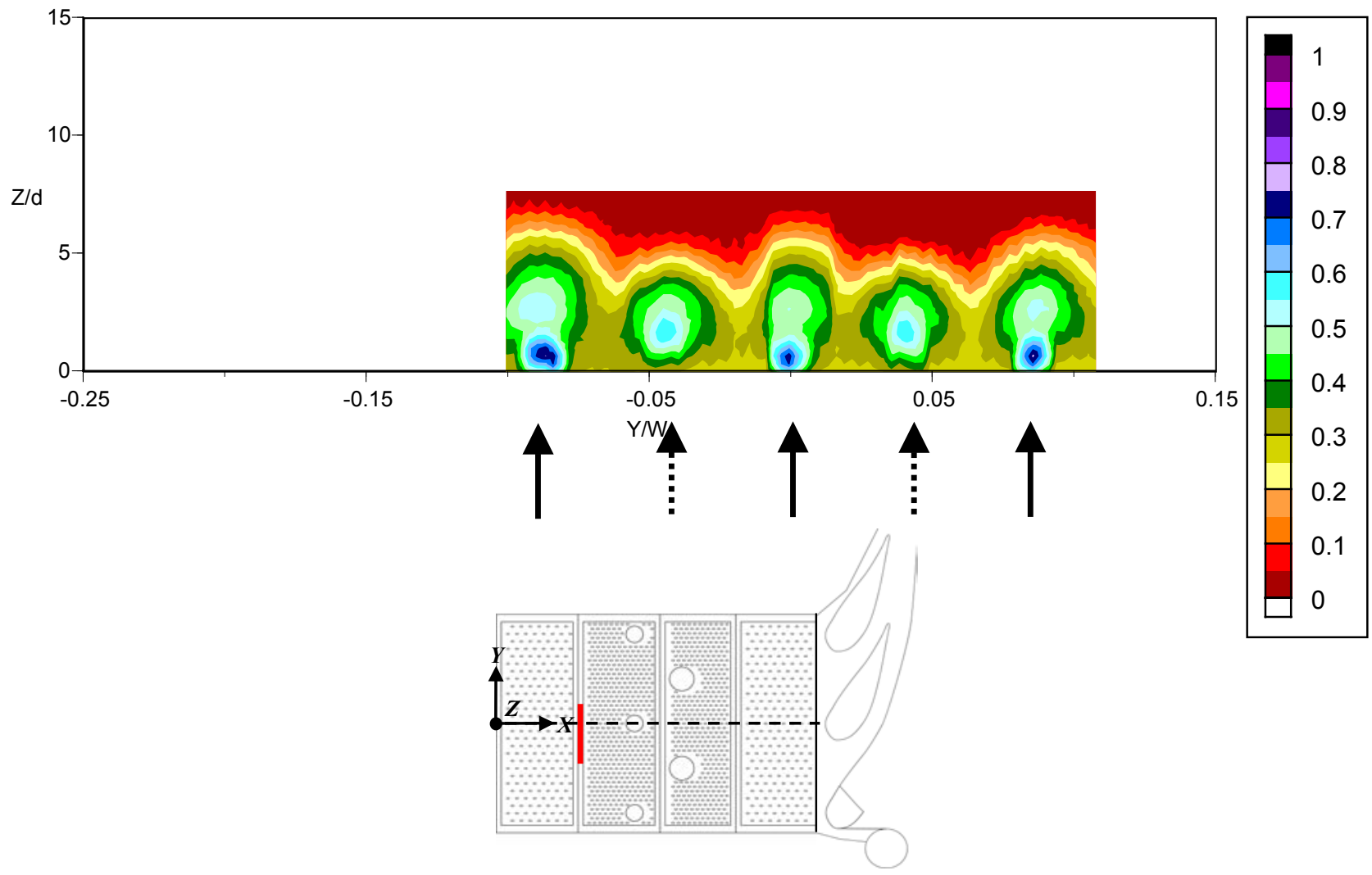

Figure 5.6 Thermal field measurements in plane $0 \mathrm{p}$ for $I=9, M=3.2$ and $D R=1.12$. 


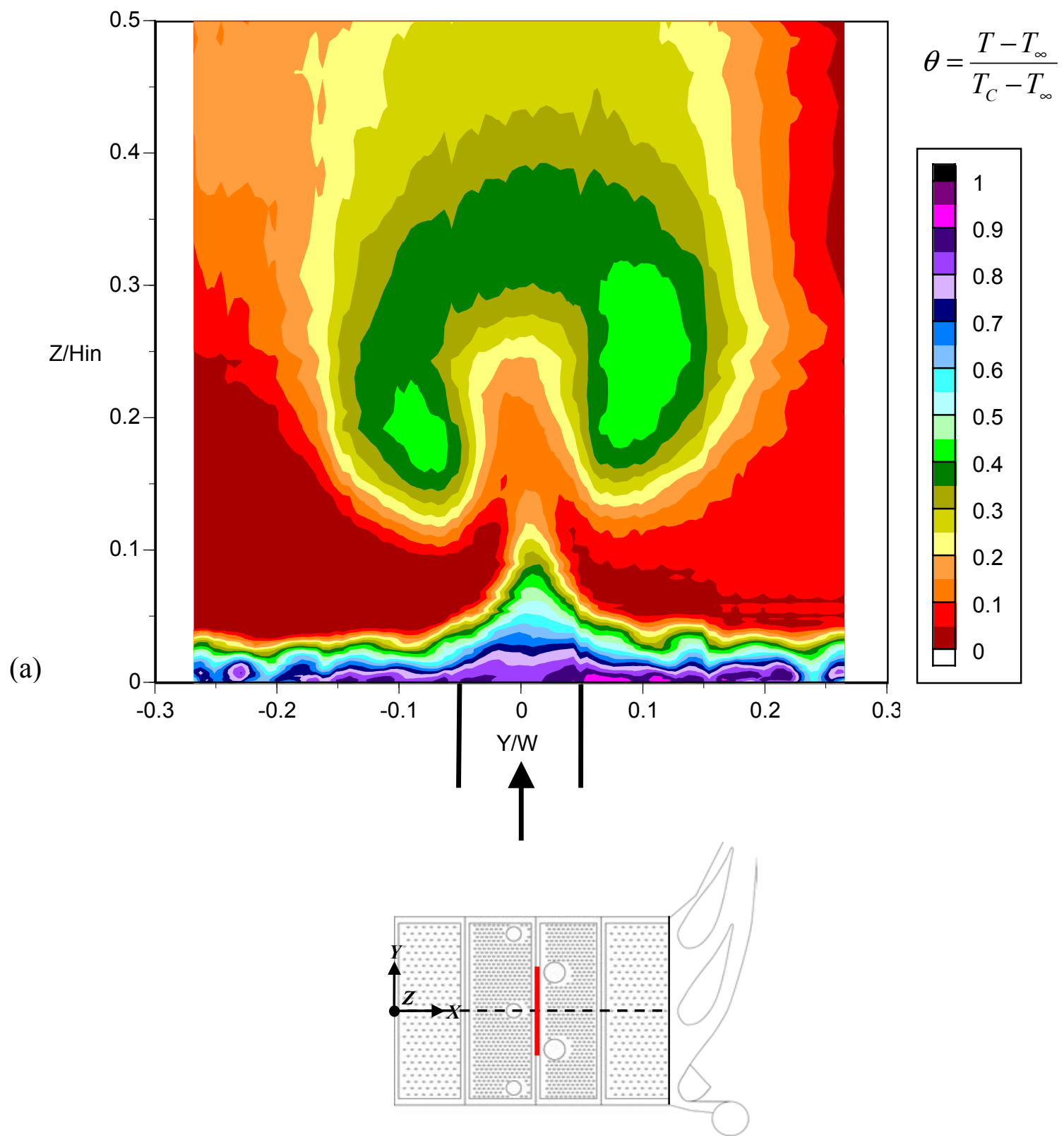

Figure 5.7 Cross-pitch temperature contours in plane 1p, downstream of dilution row one for (a) $I=3, M=1.8$ and $D R=1.08$, and (b) $I=9, M=3.2$ and $D R=$ 1.12. The dilution jet injection remained the same at $I=128$ and $M=12$, while the $D R$ was equal to those of the film-cooling jets. 


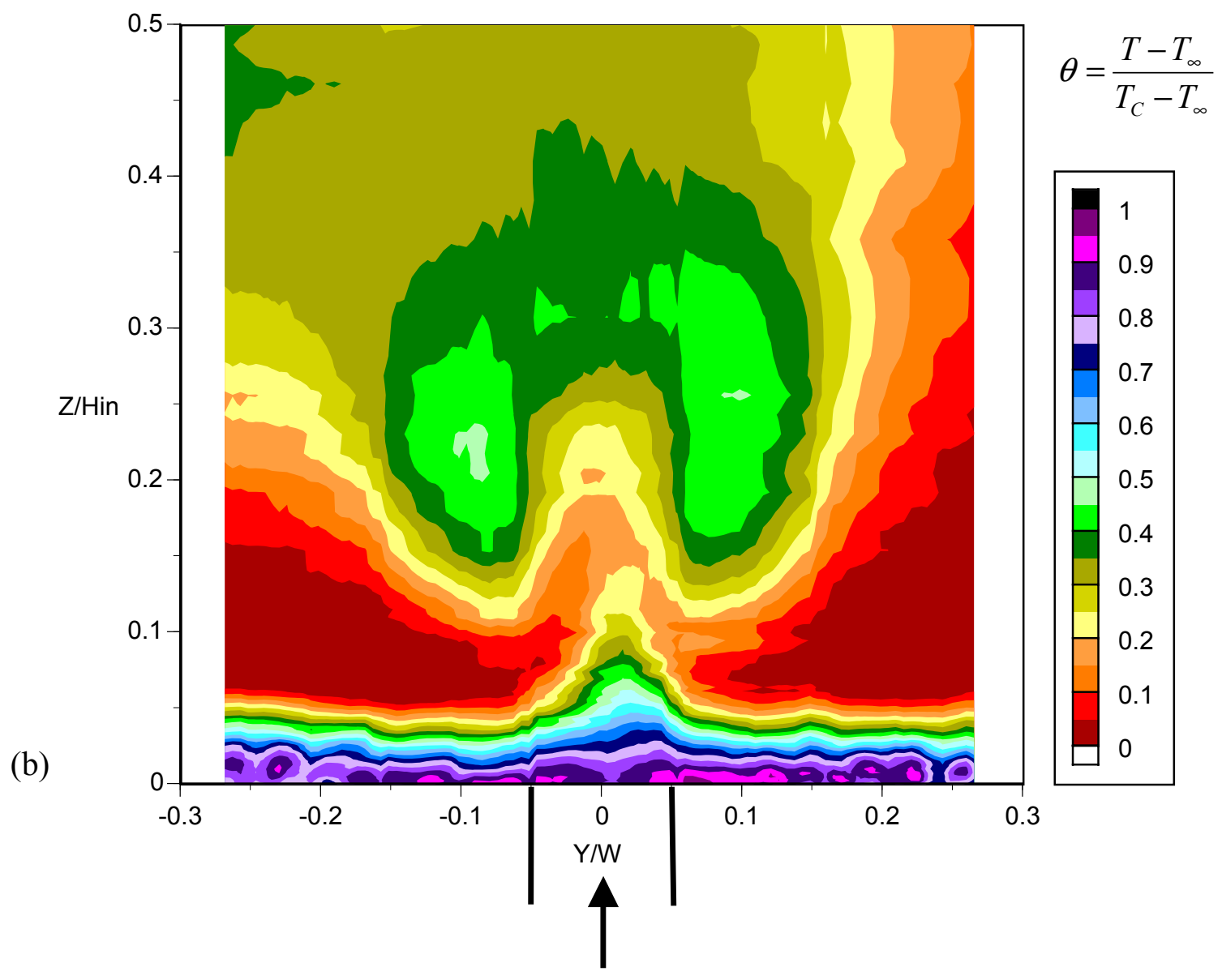

Figure 5.7 Cross-pitch temperature contours in plane $1 \mathrm{p}$, downstream of dilution row one for (a) $I=3, M=1.8$ and $D R=1.08$, and (b) $I=9, M=3.2$ and $D R=$ 1.12. The dilution jet injection remained the same at $I=128$ and $M=12$, while the $D R$ was equal to those of the film-cooling jets. 


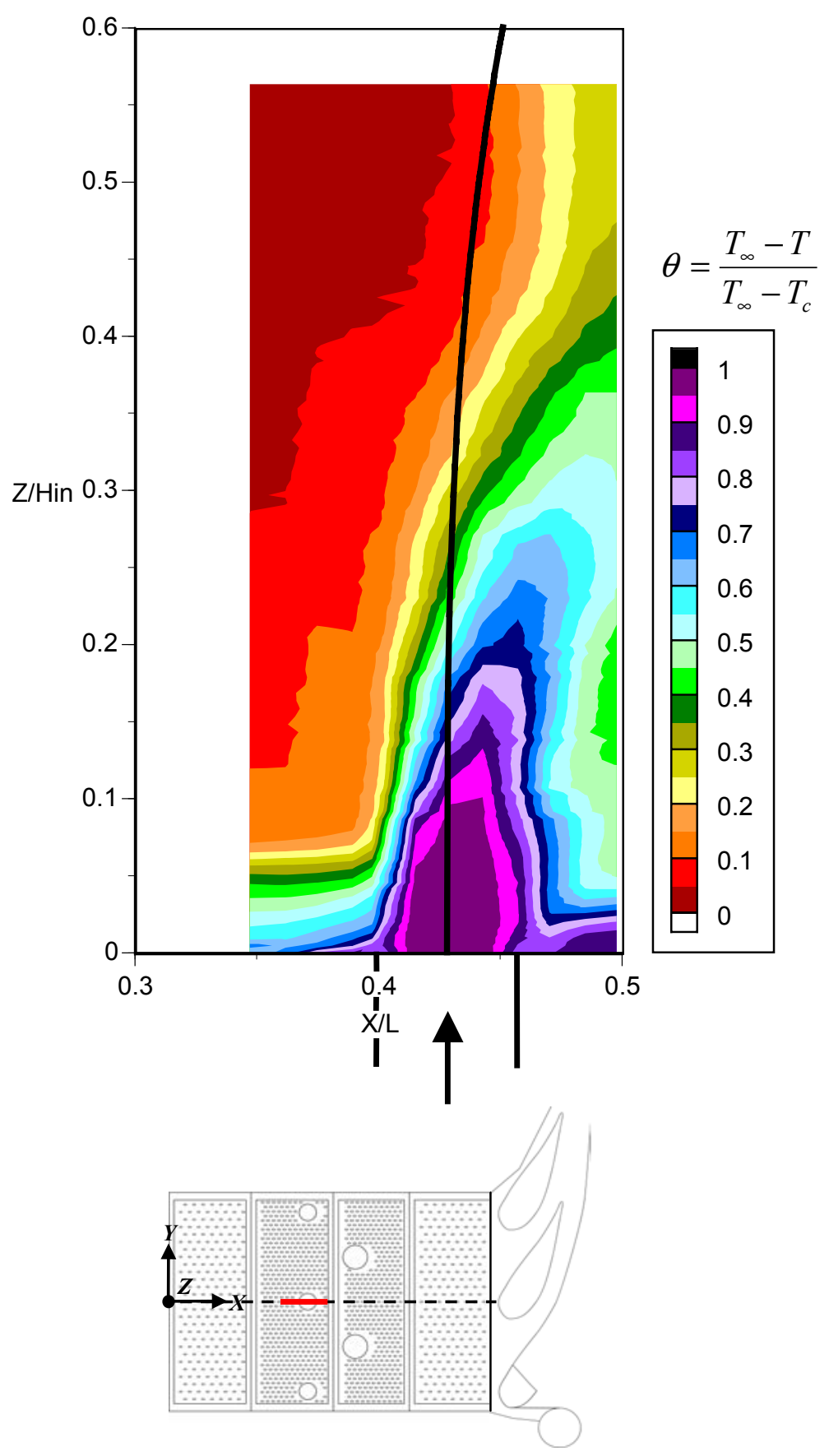

Figure 5.8 Streamwise temperature contours through a high-momentum dilution jet in plane 1s. $I=128, M=12$, and $D R=1.12$ for the dilution jet. 


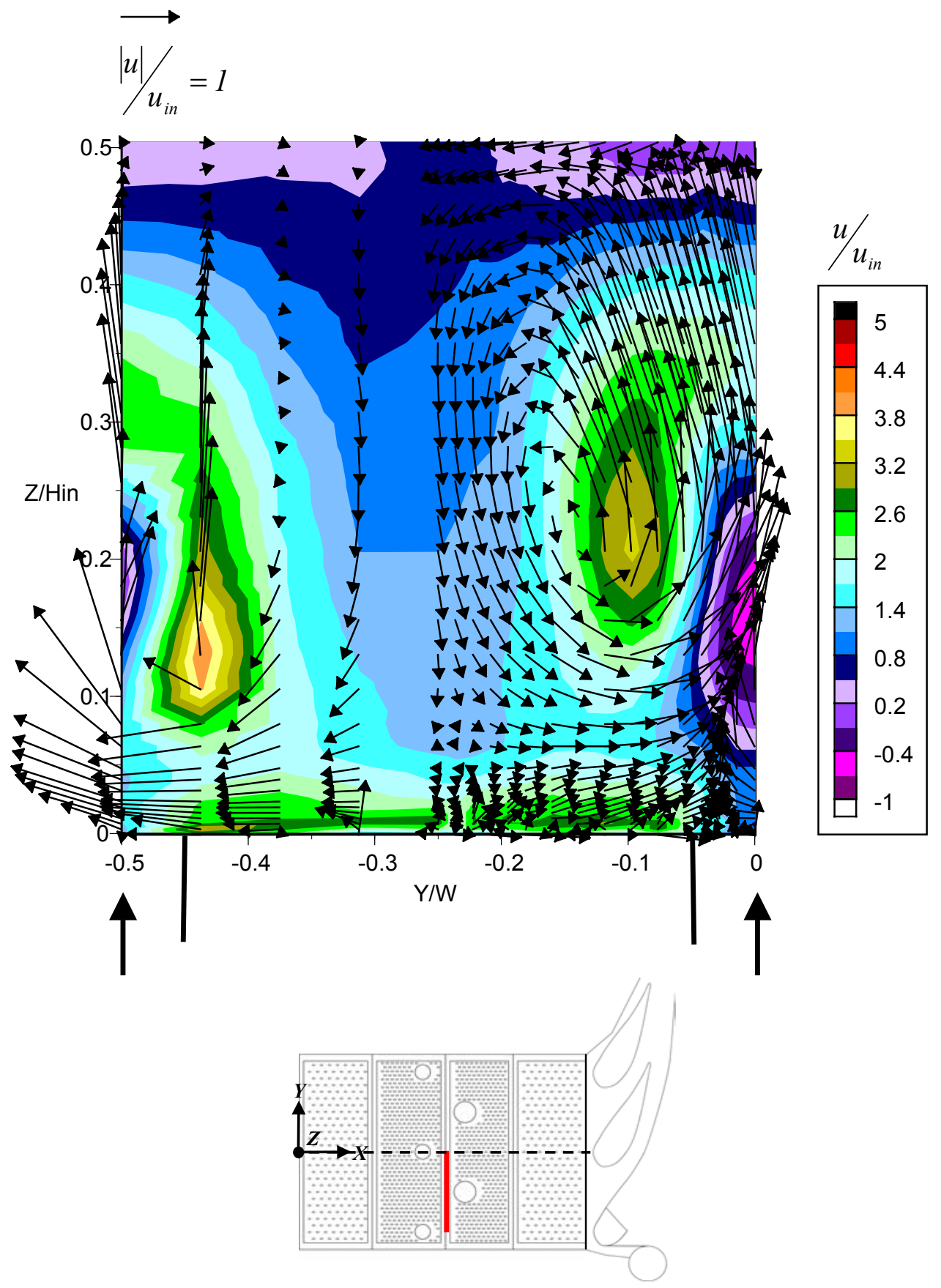

Figure 5.9 Three-component velocity measurements for plane $1 \mathrm{p}$ showing contours of $u / u_{\text {in }}$ with secondary velocity vectors $v / u_{\text {in }}$ and $w / u_{i n} . I=128, M=12$, and $D R=1.12$ for the dilution jets. 


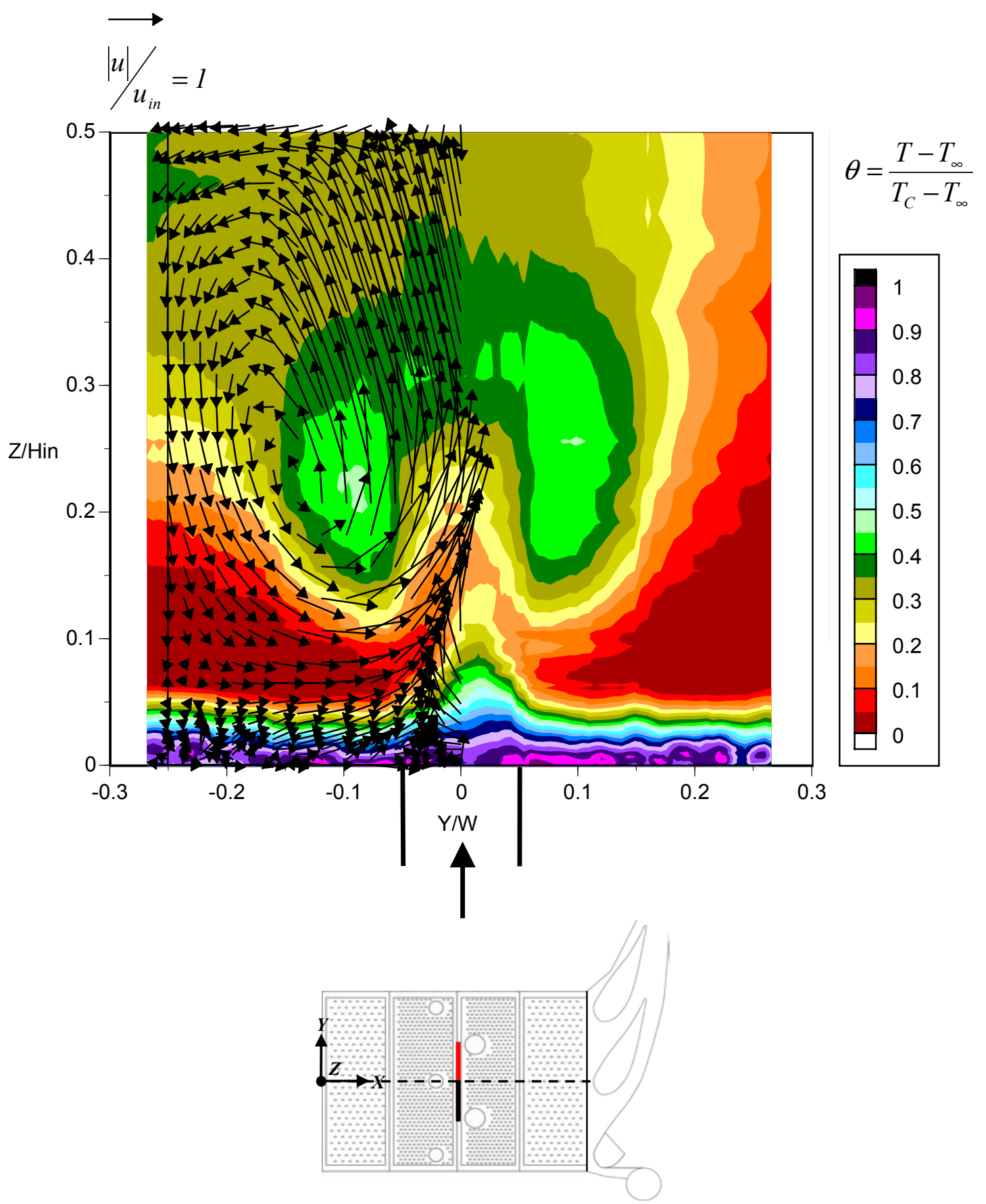

Figure 5.10 Secondary flow vectors of $v / u_{\text {in }}$ and $w / u_{\text {in }}$ with temperature contours within plane $1 \mathrm{p}$. 

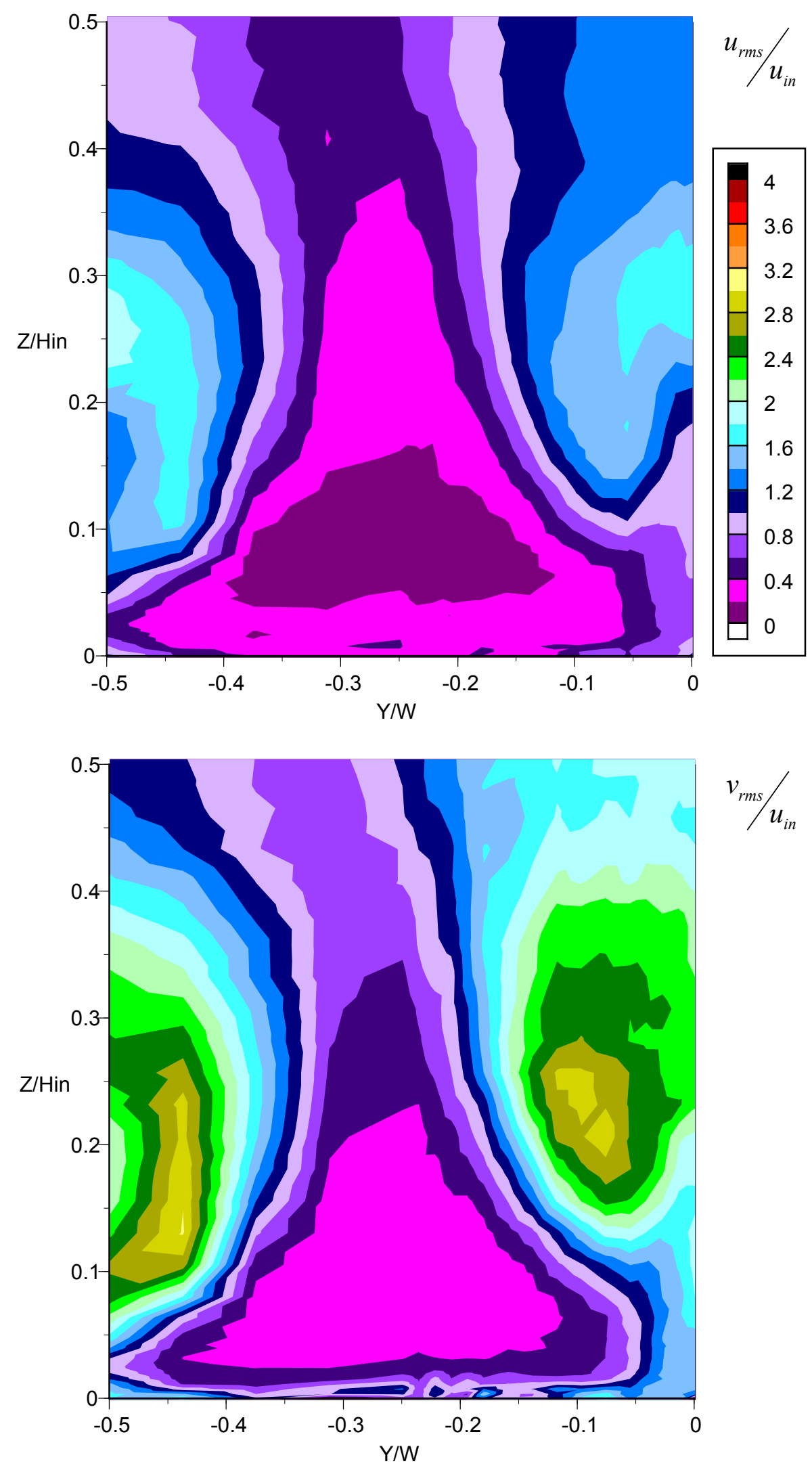

Figure 5.11 Contours of the rms fluctuations in plane $1 \mathrm{p}$; (a) $u_{r m s} / u_{i n}$, (b) $v_{r m s} / u_{\text {in }}$ and (c) $w_{r m s} / u_{i n} . I=128, M=12$, and $D R=1.12$ for the dilution jets. 


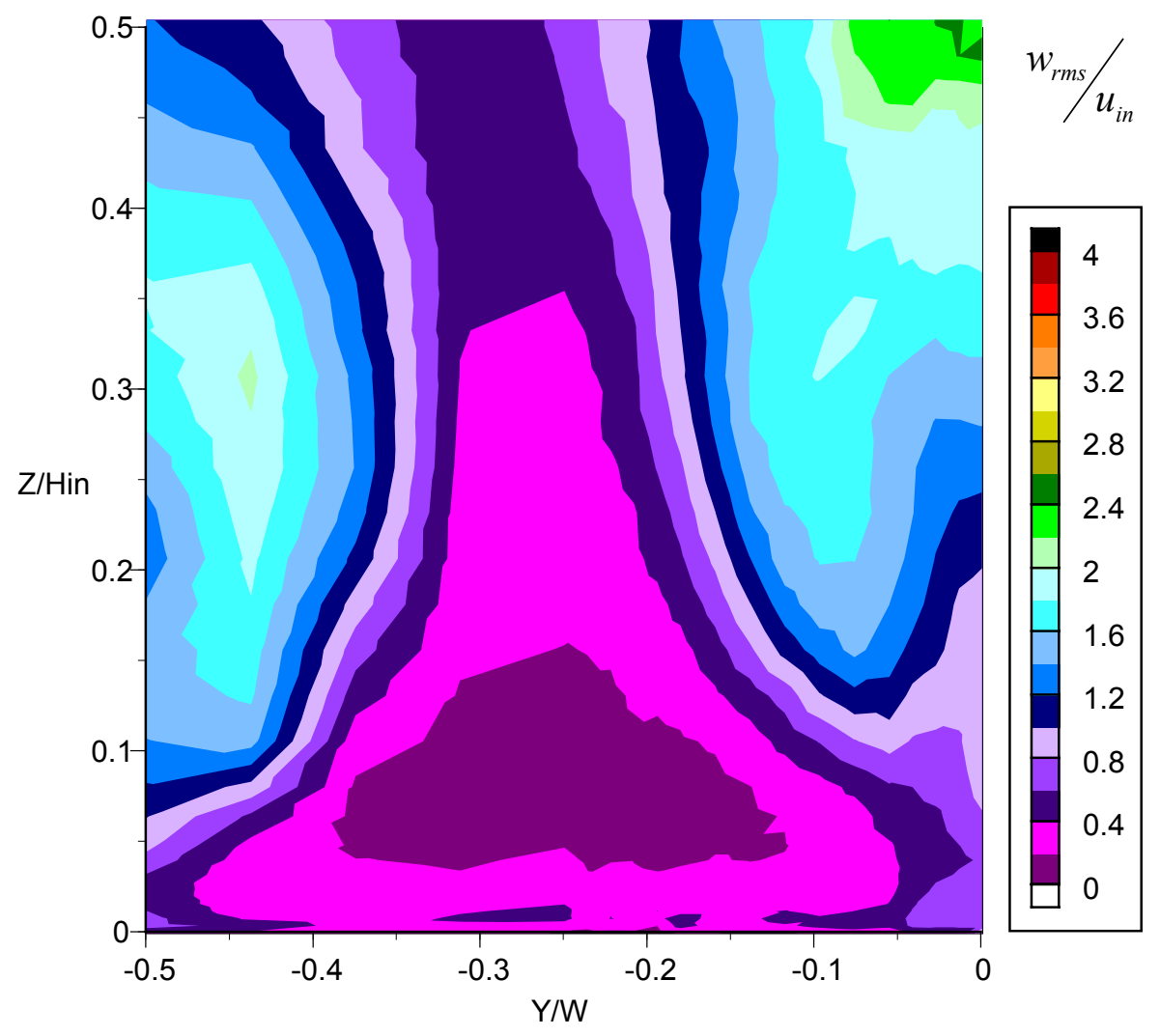

Figure 5.11 Contours of the rms fluctuations in plane 1p; (a) $u_{r m s} / u_{i n}$, (b) $v_{r m s} / u_{\text {in }}$ and (c) $w_{r m s} / u_{i n} . I=128, M=12$, and $D R=1.12$ for the dilution jets. 


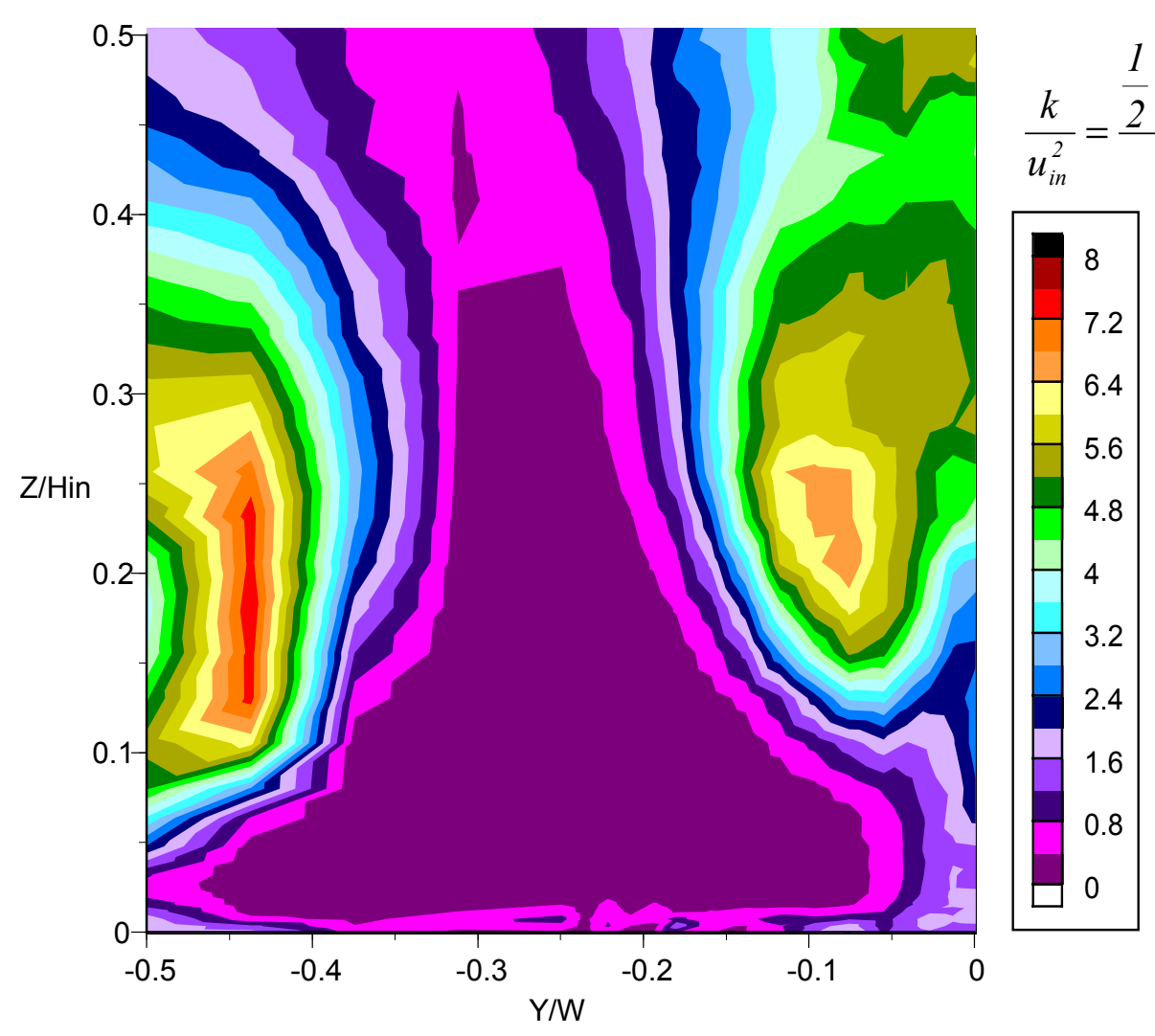

Figure 5.12 Contours of the normalized combustor turbulent kinetic energy, $k / u_{i n}^{2}$, in plane $1 \mathrm{p}$. 


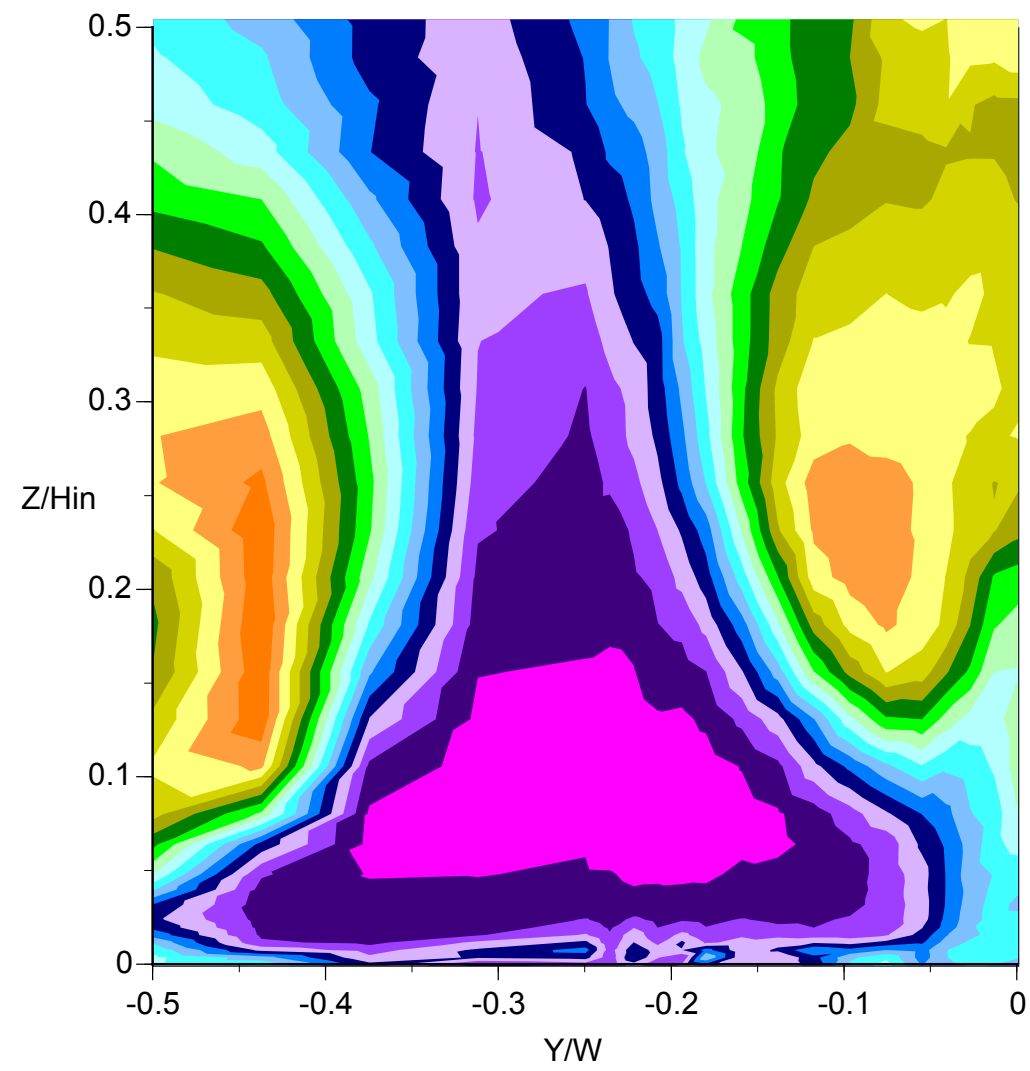

$T L=\frac{\sqrt{\frac{1}{3}\left(u_{r m s}^{2}+v_{r m s}^{2}+w_{r m s}^{2}\right)}}{u_{i n}}$

\begin{tabular}{|l|l|}
2.5 \\
2.25 \\
2 \\
1.75 \\
1.5 \\
1.25 \\
1 \\
0.75 \\
0.5 \\
0.25 \\
0 \\
\hline
\end{tabular}

Figure 5.13 Contours of the turbulence levels, $T L$, in plane 1p.

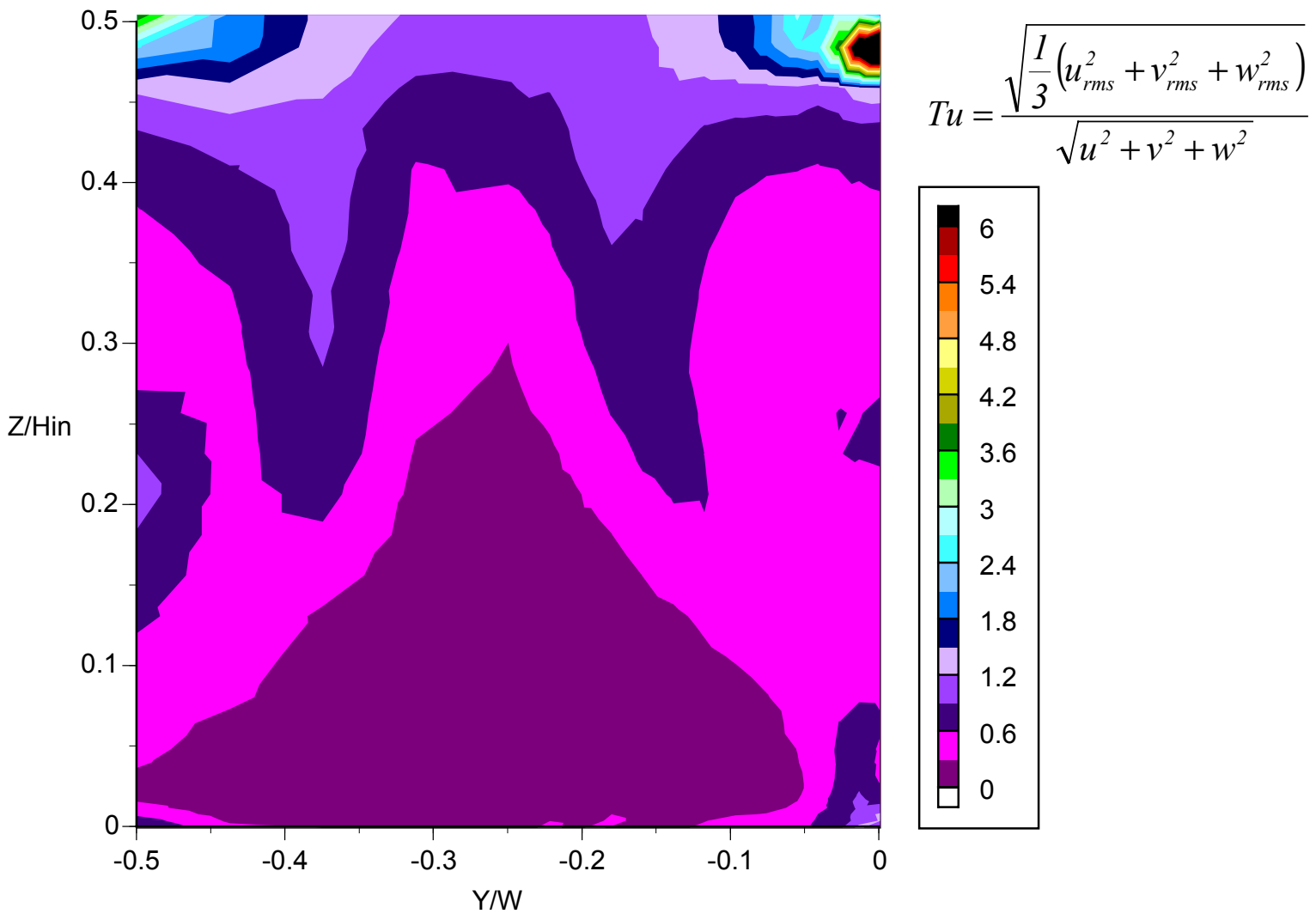

Figure 5.14 Contours showing the local turbulence levels, $T u$, within plane $1 \mathrm{p}$ (normalized mass-averaged velocity $u / u_{i n}=1.55$ ). 


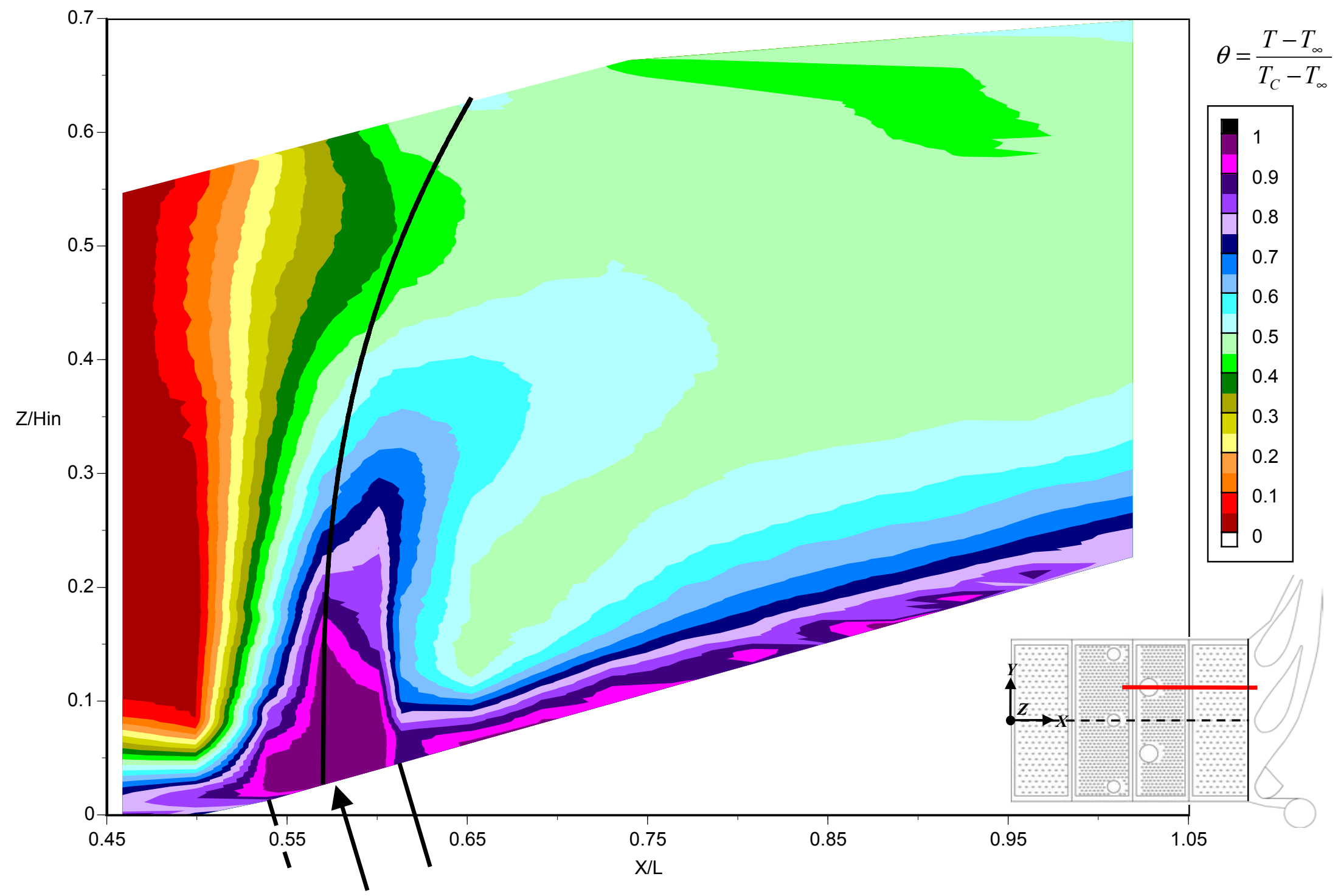

Figure 5.15 Streamwise normalized temperature contours for plane $2 \mathrm{~s}$, beginning slightly before the second dilution row and extending through the combustor exit. The dilution jet has an $I=32, M=6$, and $D R=1.12$. 


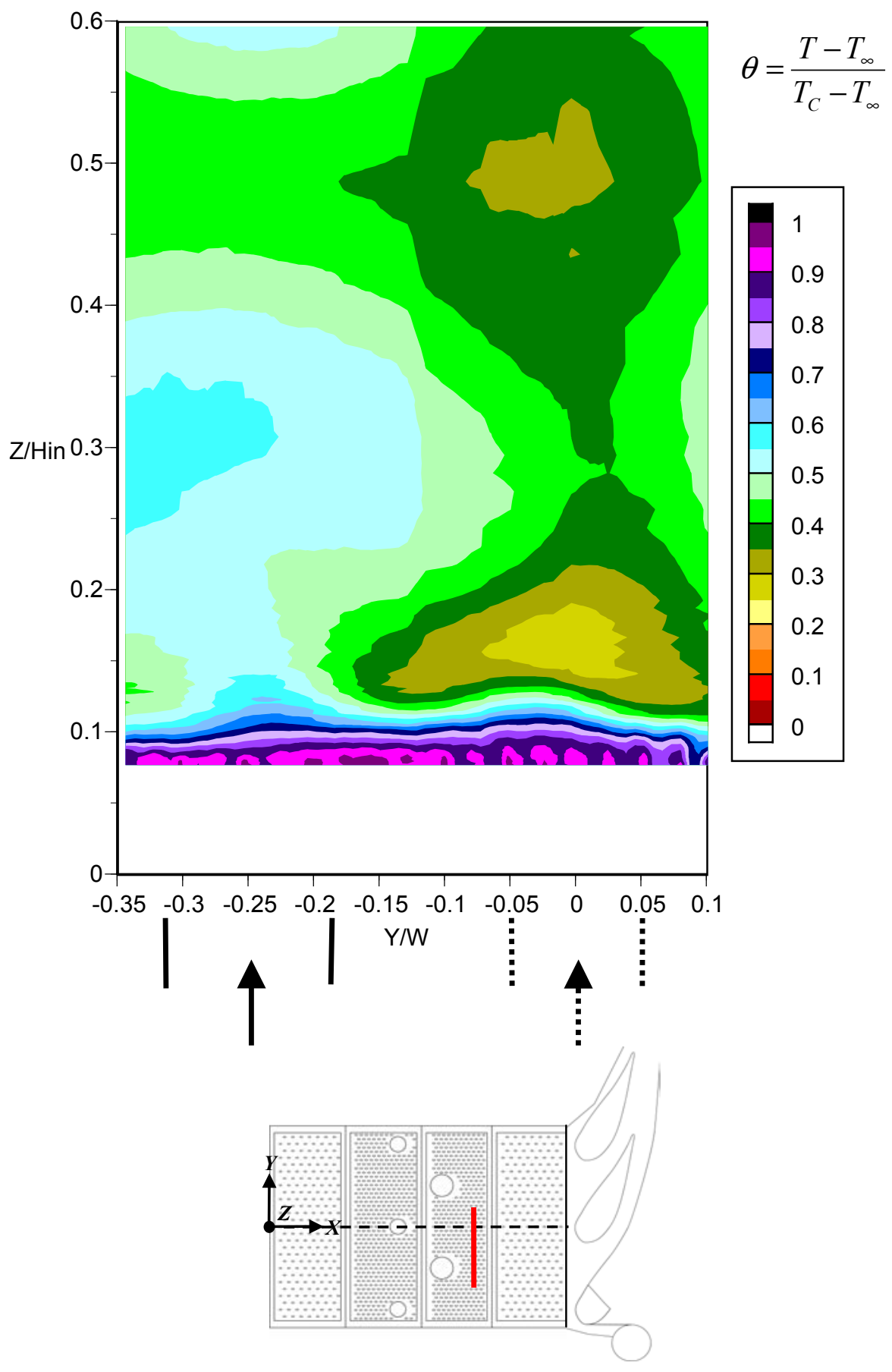

Figure 5.16 Temperature contours for plane $2 \mathrm{p}$ at one dilution hole diameter downstream $\left(1 D_{2}\right)$. The first row of dilution has an $I=128$ and $M=12$, while the second row has an $I=32$ and $M=6$. The $D R=1.12$ for the filmcooling and both rows of dilution. 


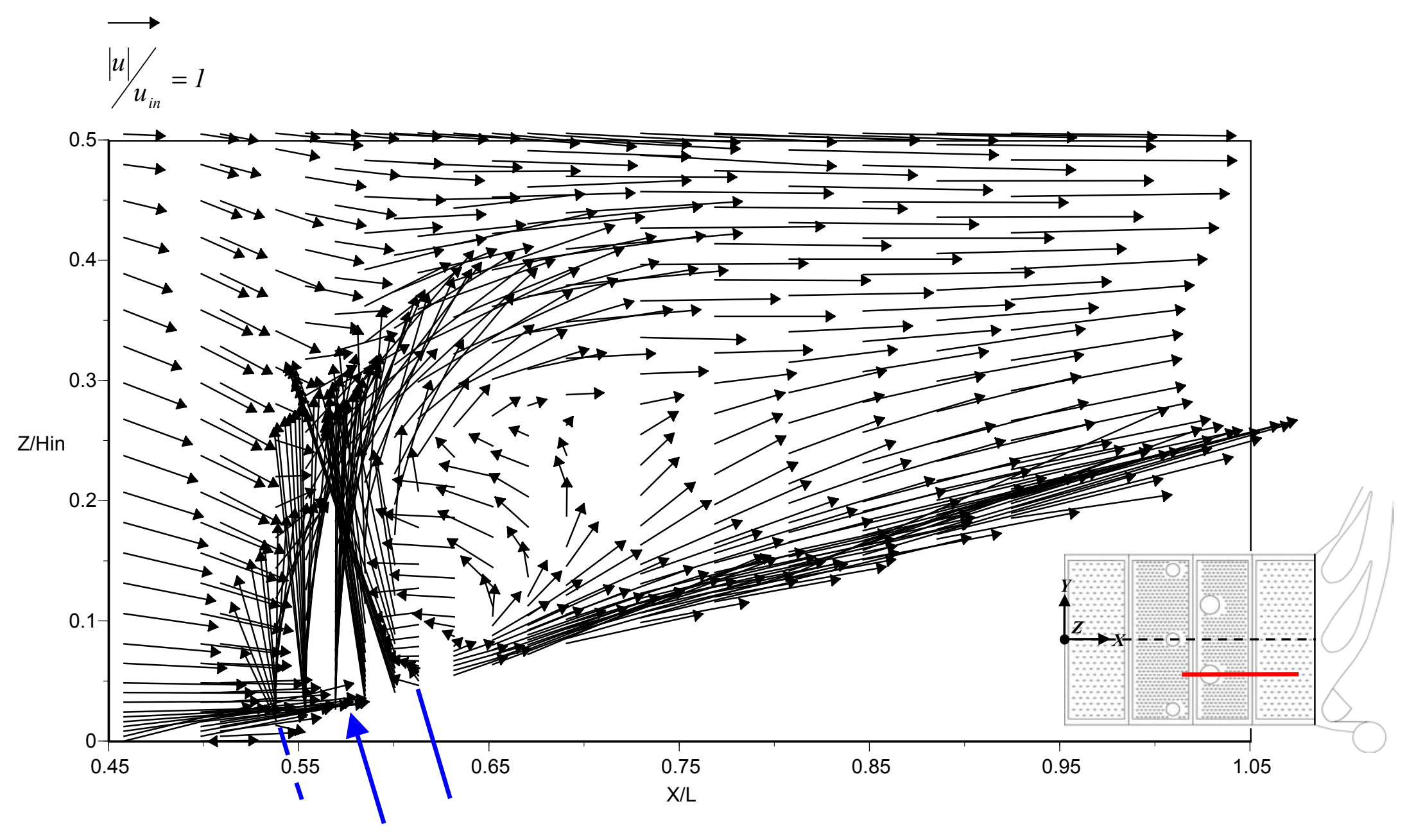

Figure 5.17 Streamwise, $u / u_{i n}$, and vertical, $w / u_{i n}$, vectors for plane $2 \mathrm{~s} . I=32, M=6$, and $D R=1.12$ for the dilution jet. 


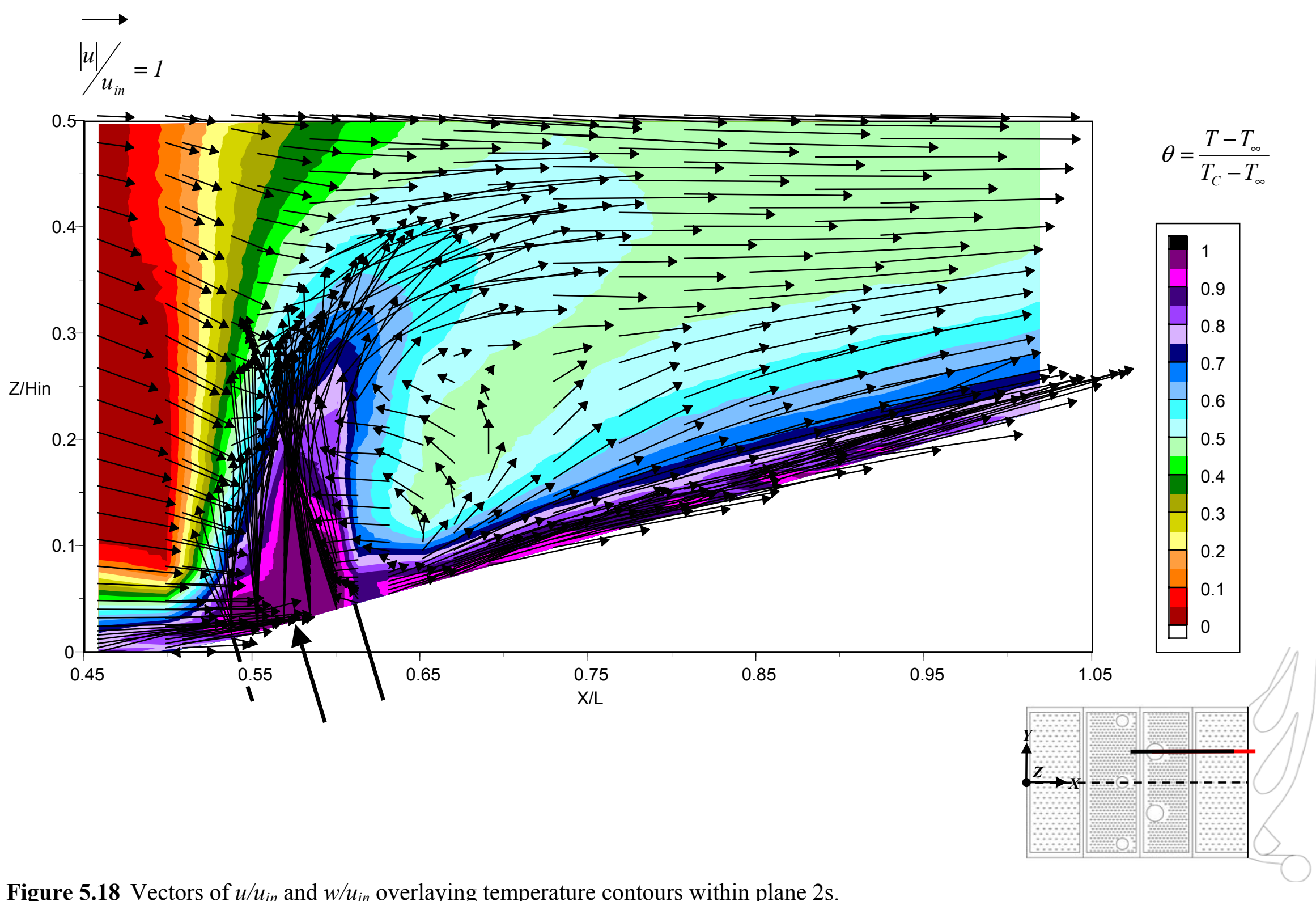

Figure 5.18 Vectors of $u / u_{\text {in }}$ and $w / u_{\text {in }}$ overlaying temperature contours within plane $2 \mathrm{~s}$. $I=32, M=6$, and $D R=1.12$ for the dilution jet. 


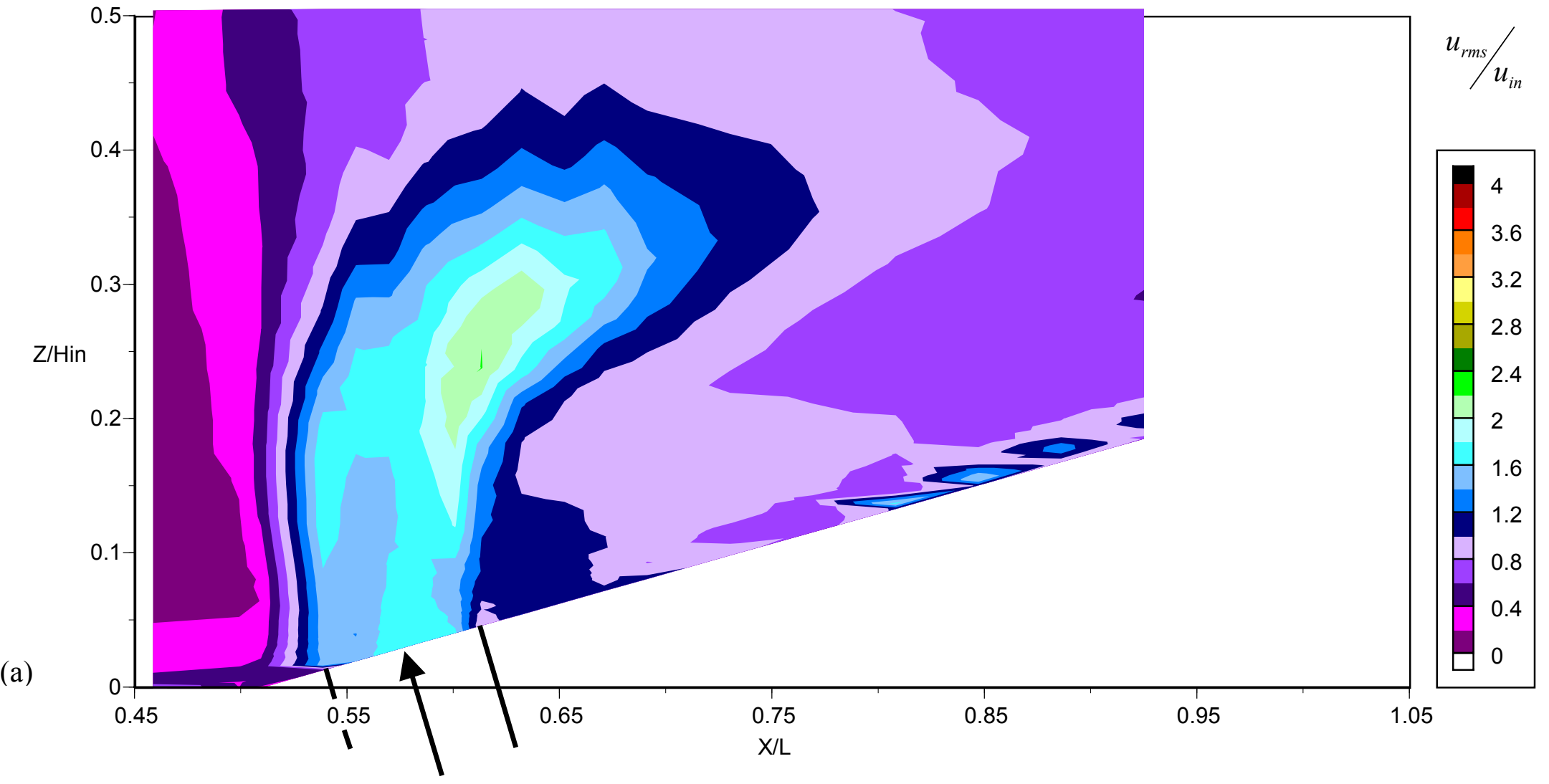

Figure 5.19 Contours of the rms fluctuations for the flow vectors in plane $2 \mathrm{~s}$; (a) $u_{r m s} / u_{\text {in }}$ and (b) $w_{r m s} / u_{i n}$. The dilution jet has an $I=32, M=6$, and $D R=1.12$. 


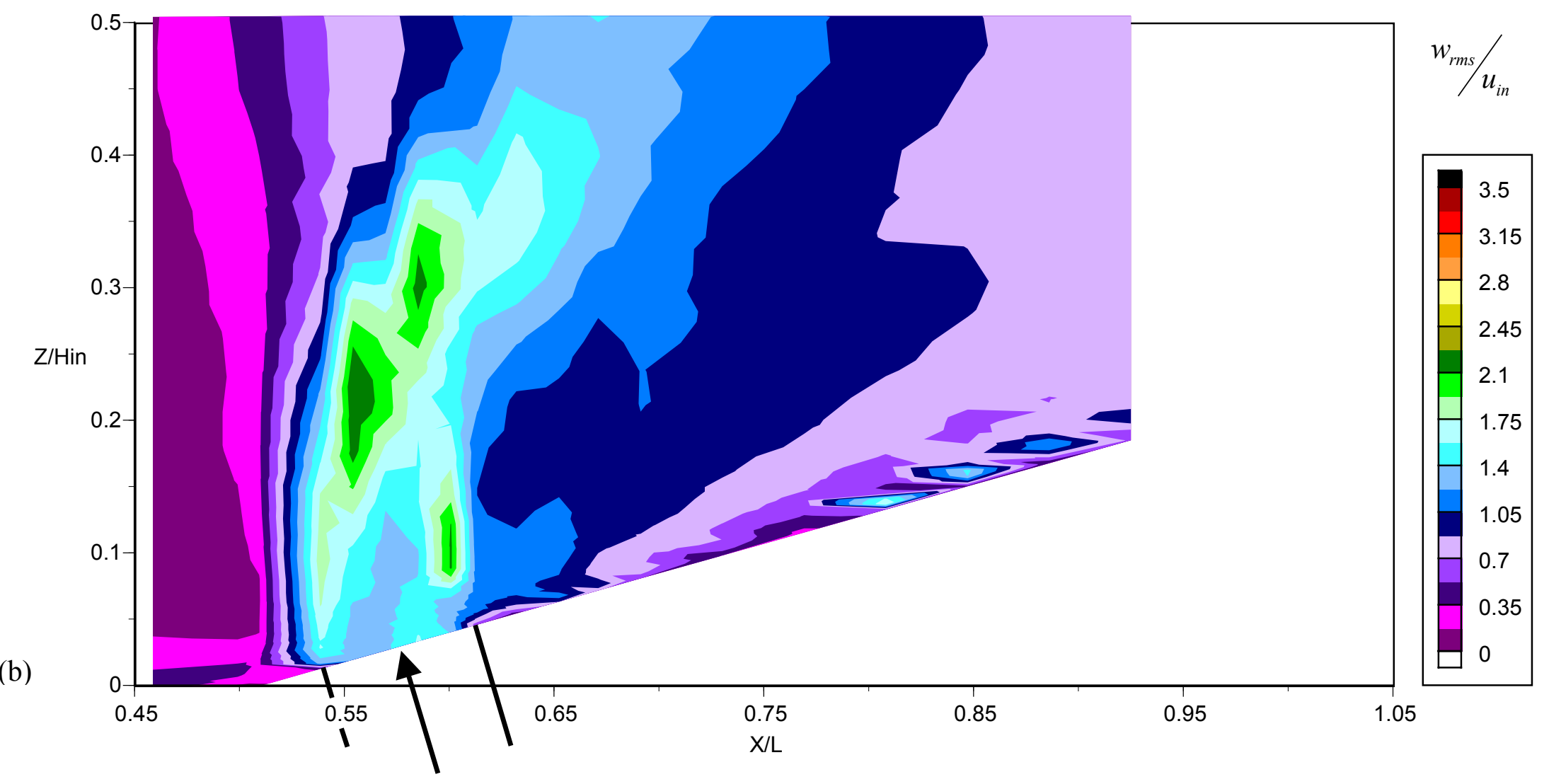

Figure 5.19 Contours of the rms fluctuations for the flow vectors in plane $2 \mathrm{~s}$; (a) $u_{r m s} / u_{\text {in }}$ and (b) $w_{r m s} / u_{i n}$. The dilution jet has an $I=32, M=6$, and $D R=1.12$. 


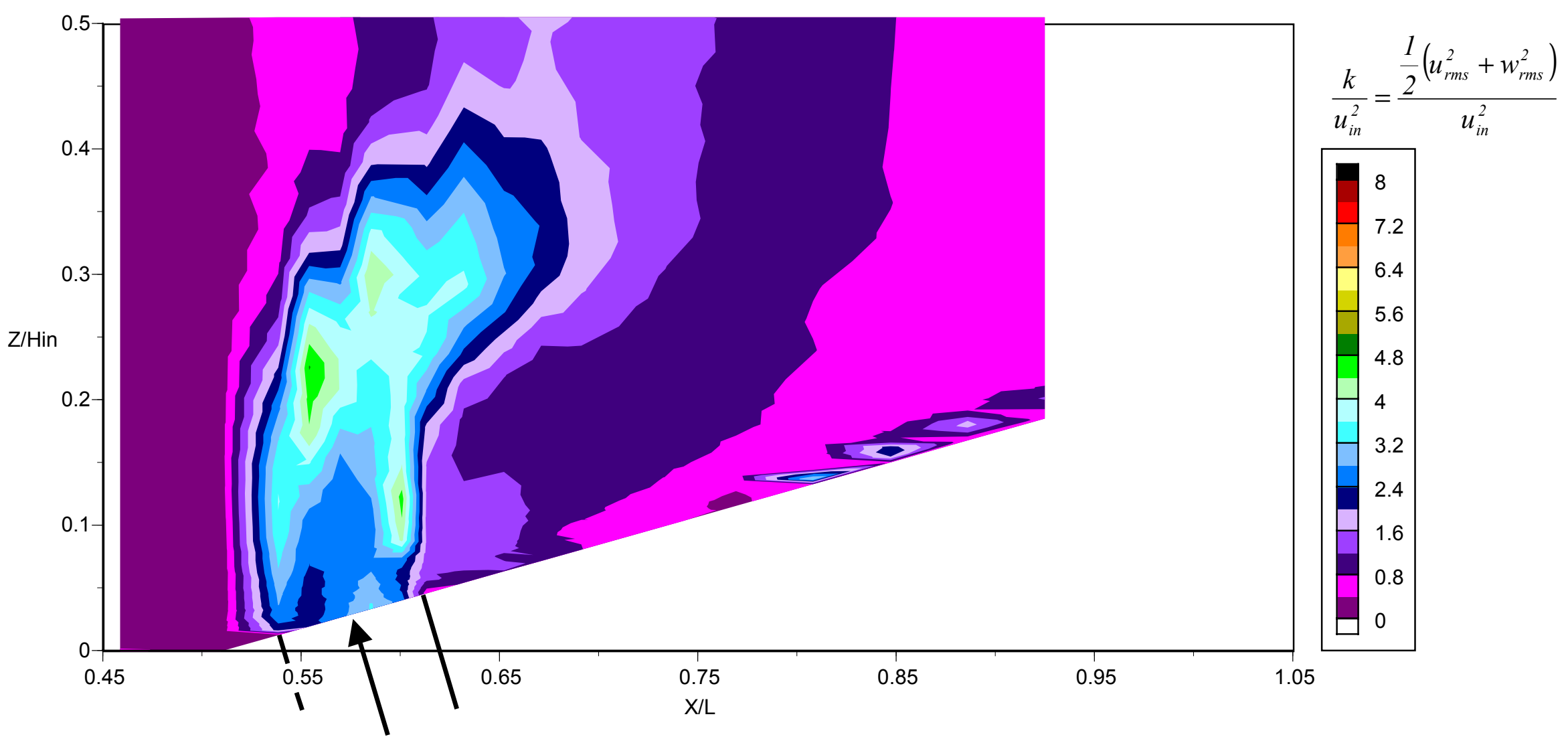

Figure 5.20 Contours of the normalized combustor turbulent kinetic energy, $k / u_{i n}^{2}$, in plane 2 s. 


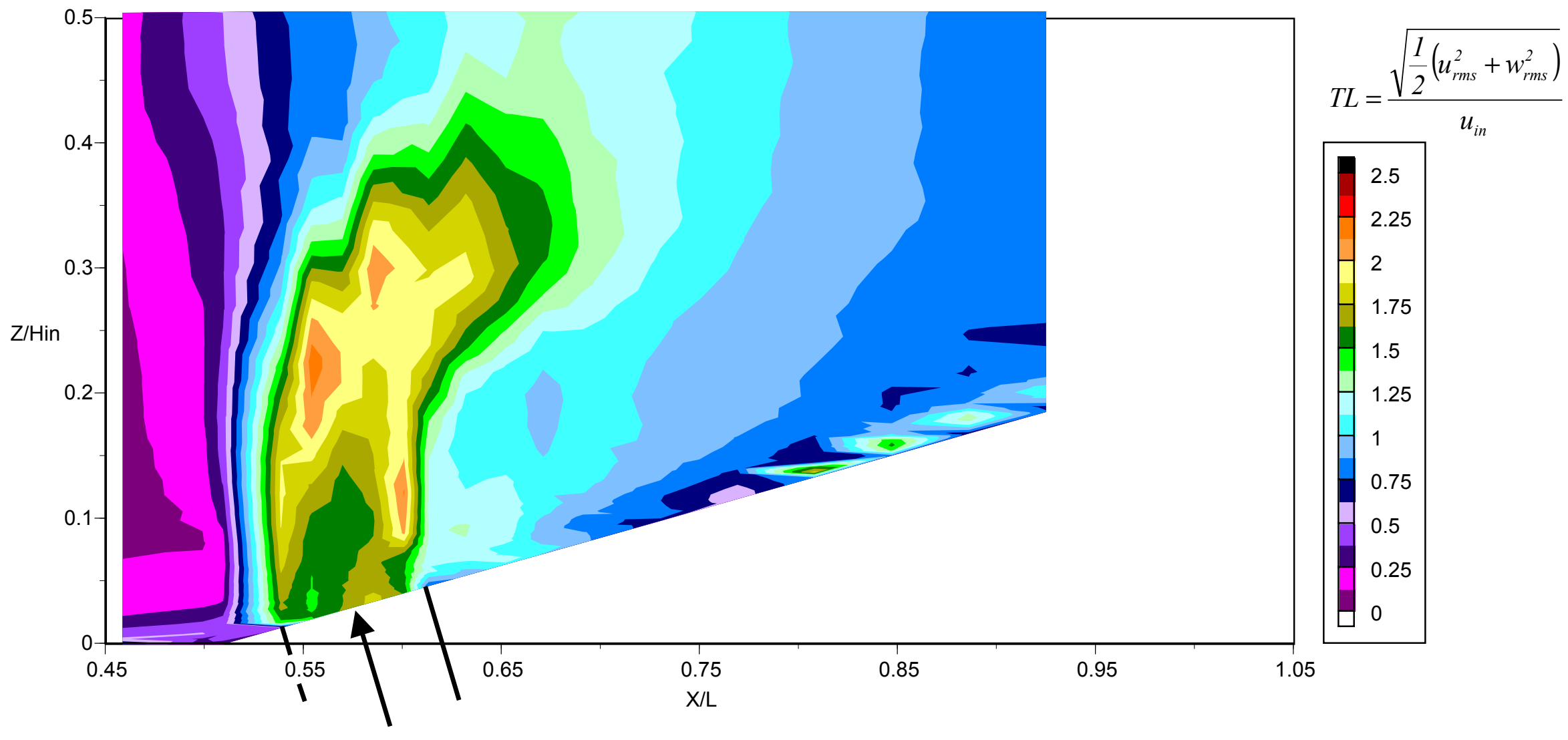

Figure 5.21 Turbulence levels, $T L$, within plane $2 \mathrm{~s}$. 


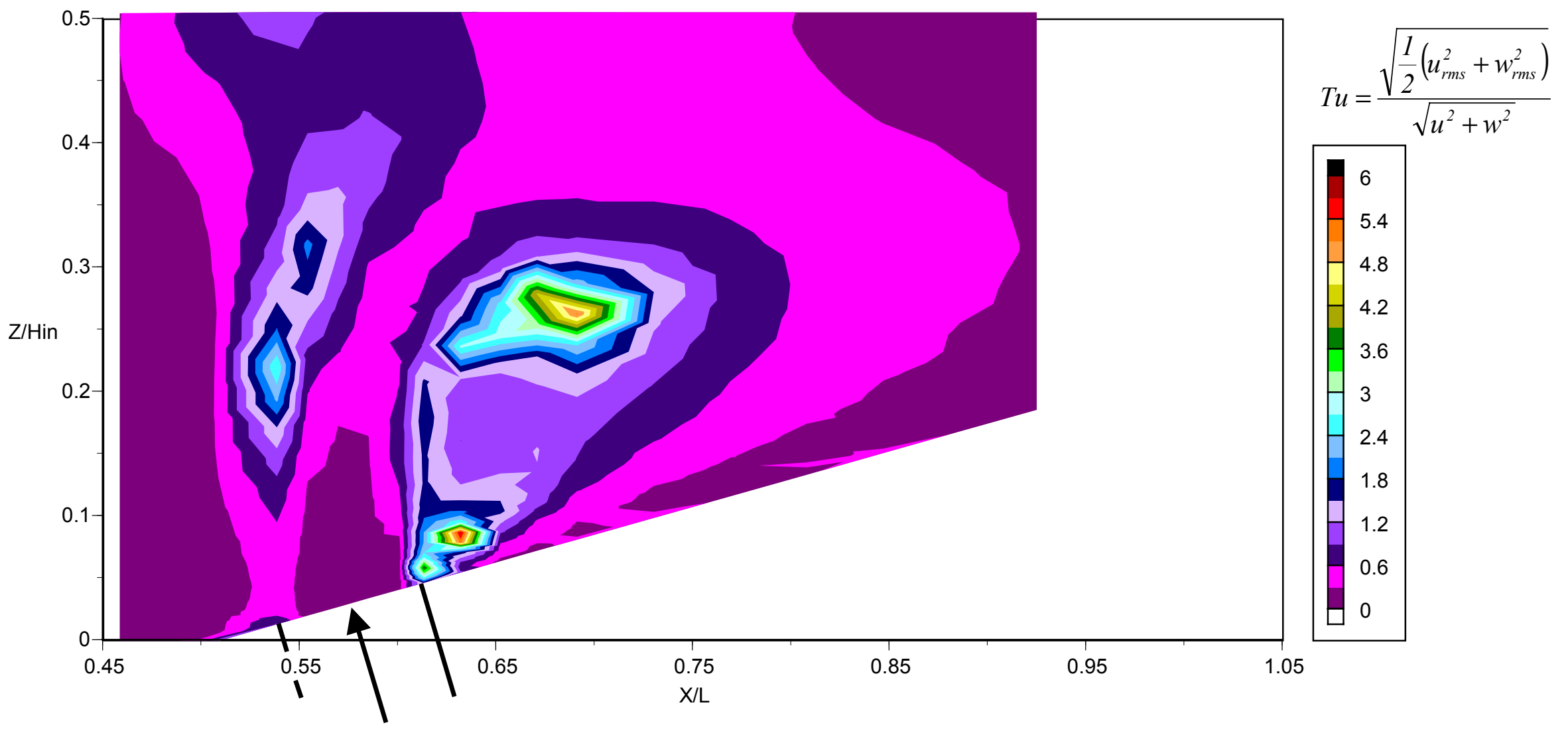

Figure 5.22 Contours of the local turbulence level, $T u$, within plane $2 \mathrm{~s}$. 


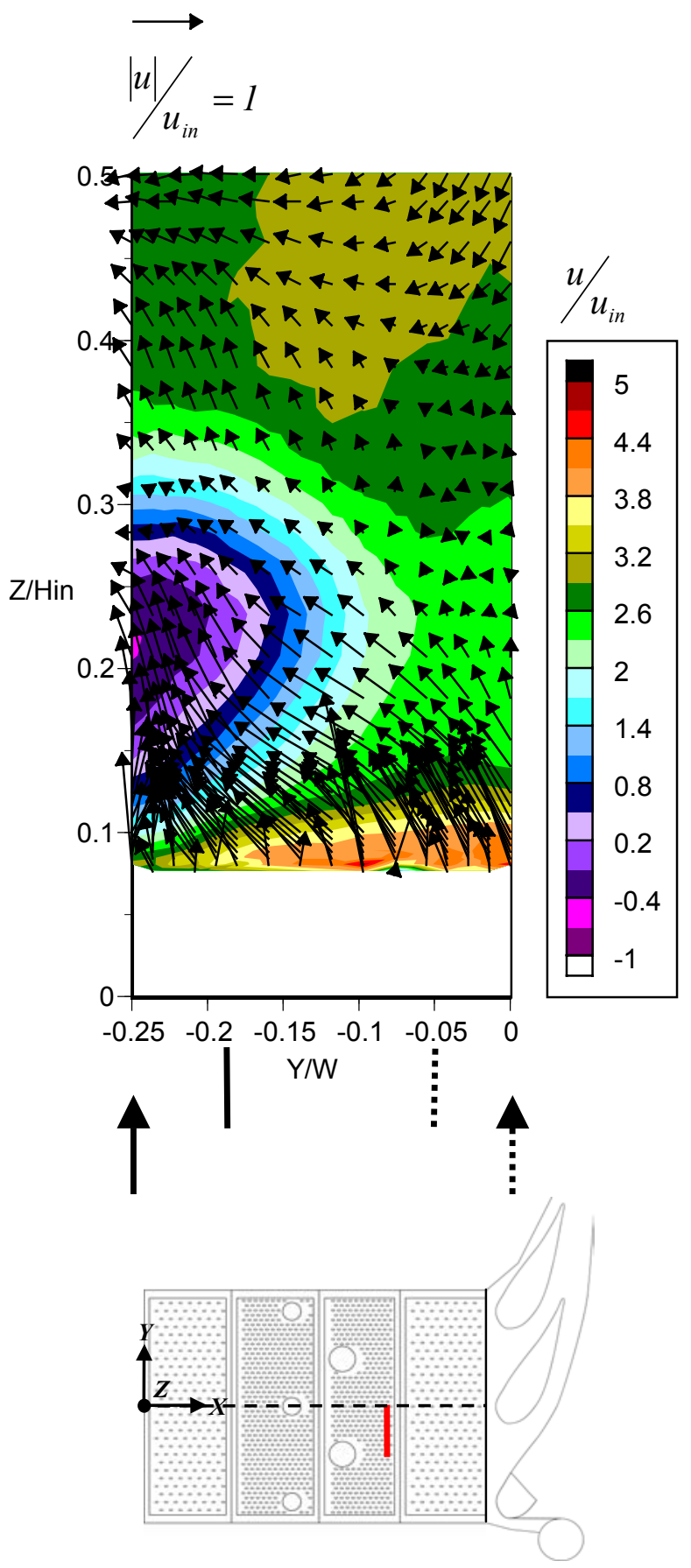

Figure 5.23 Three-component velocity measurements for plane $2 p$, post-dilution row two; contours of $u / u_{i n}$ and vectors of $v / u_{i n}$ and $w / u_{i n}$. The second row dilution jet (on the left) has an $I=32, M=6$, and $D R=1.12$, while the first row dilution jet (on the right) has an $I=128, M=12$, and $D R=1.12$. 


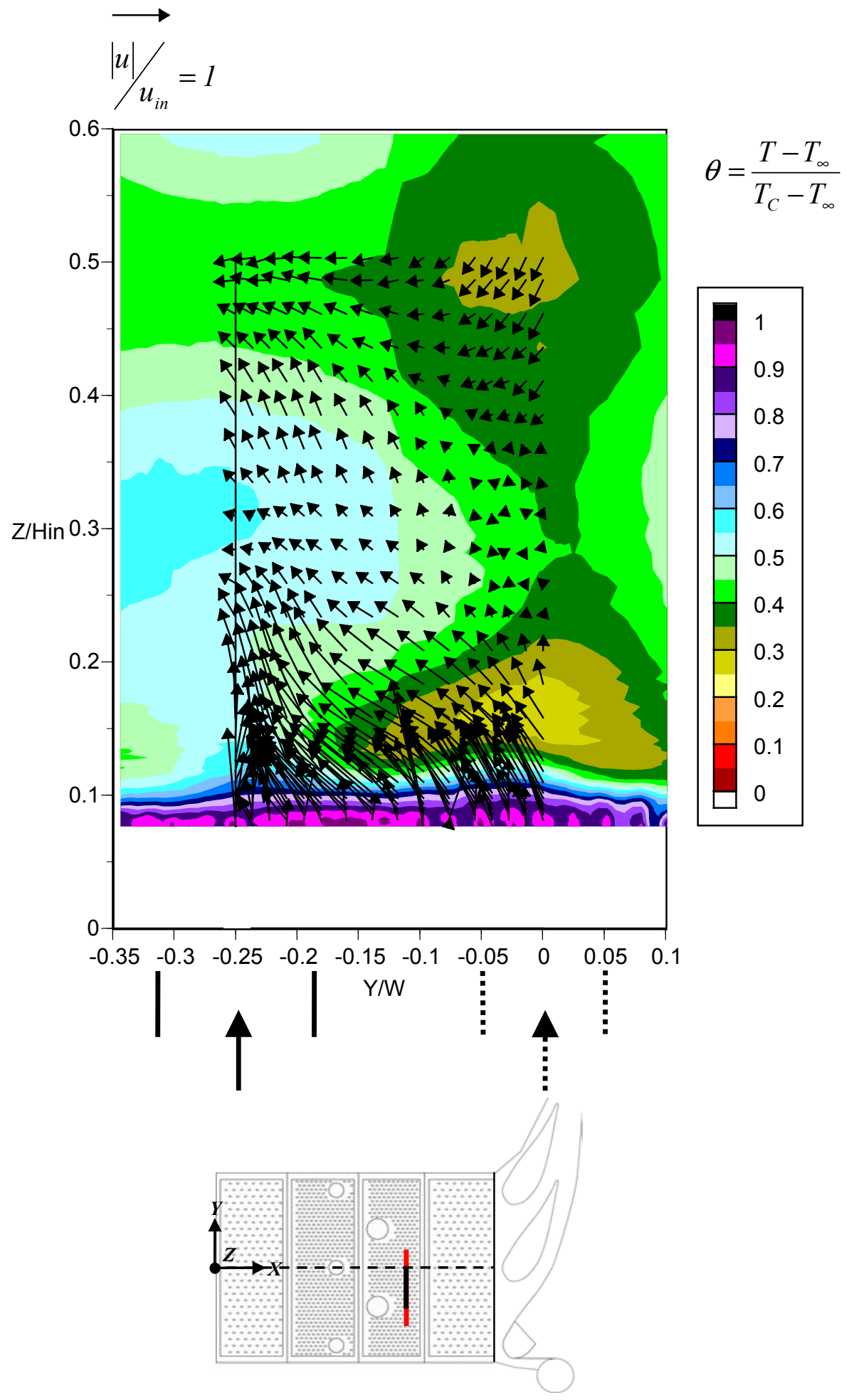

Figure 5.24 Vectors of $v / u_{\text {in }}$ and $w / u_{\text {in }}$ overlying the temperature contours within plane $2 \mathrm{p}$ (post-dilution row two). $I=32, M=6$, and $D R=1.12$ for the second row dilution jet, and $I=128, M=12$, and $D R=1.12$ for the first row dilution jet. 

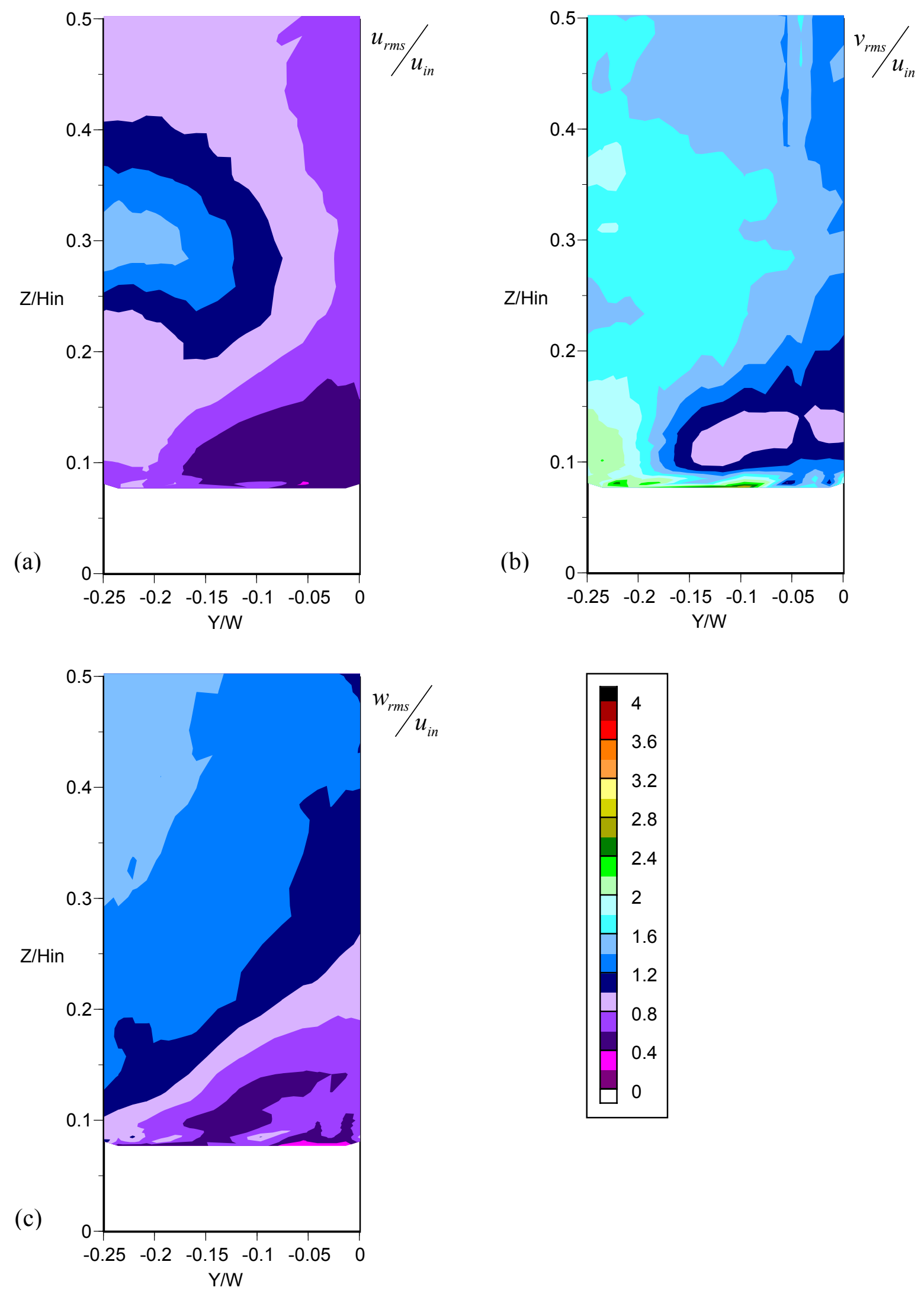

Figure 5.25 Contours of the rms fluctuations for the flow field in plane $2 \mathrm{p}$; (a) $u_{r m s} / u_{i n}$, (b) $v_{r m s} / u_{i n}$, and (c) $w_{r m s} / u_{\text {in }}$ (second row $I=32, M=6$, and $D R=1.12$ ). 


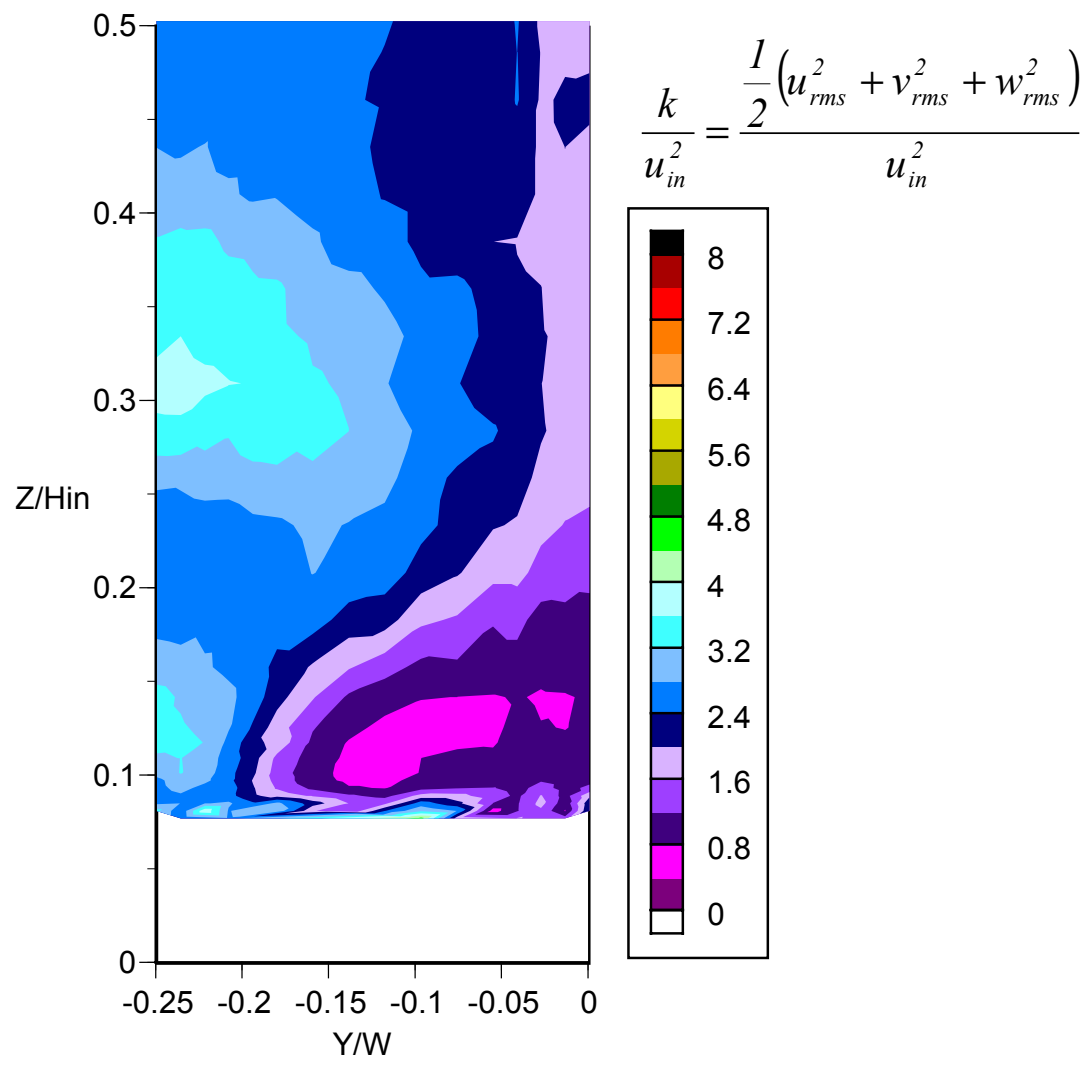

Figure 5.26 Contours of the combustor turbulent kinetic energy, $k / u_{i n}^{2}$, in plane $2 p$. 


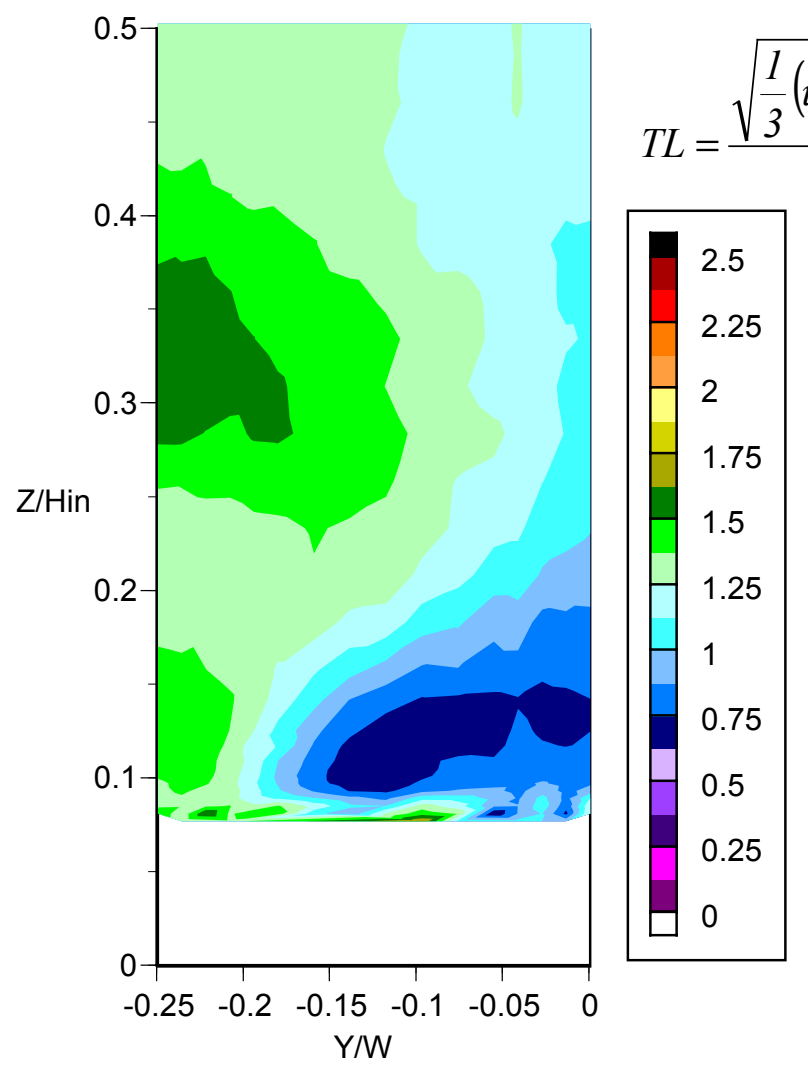

Figure 5.27 Contours of the turbulence levels, $T L$, in plane 2p.

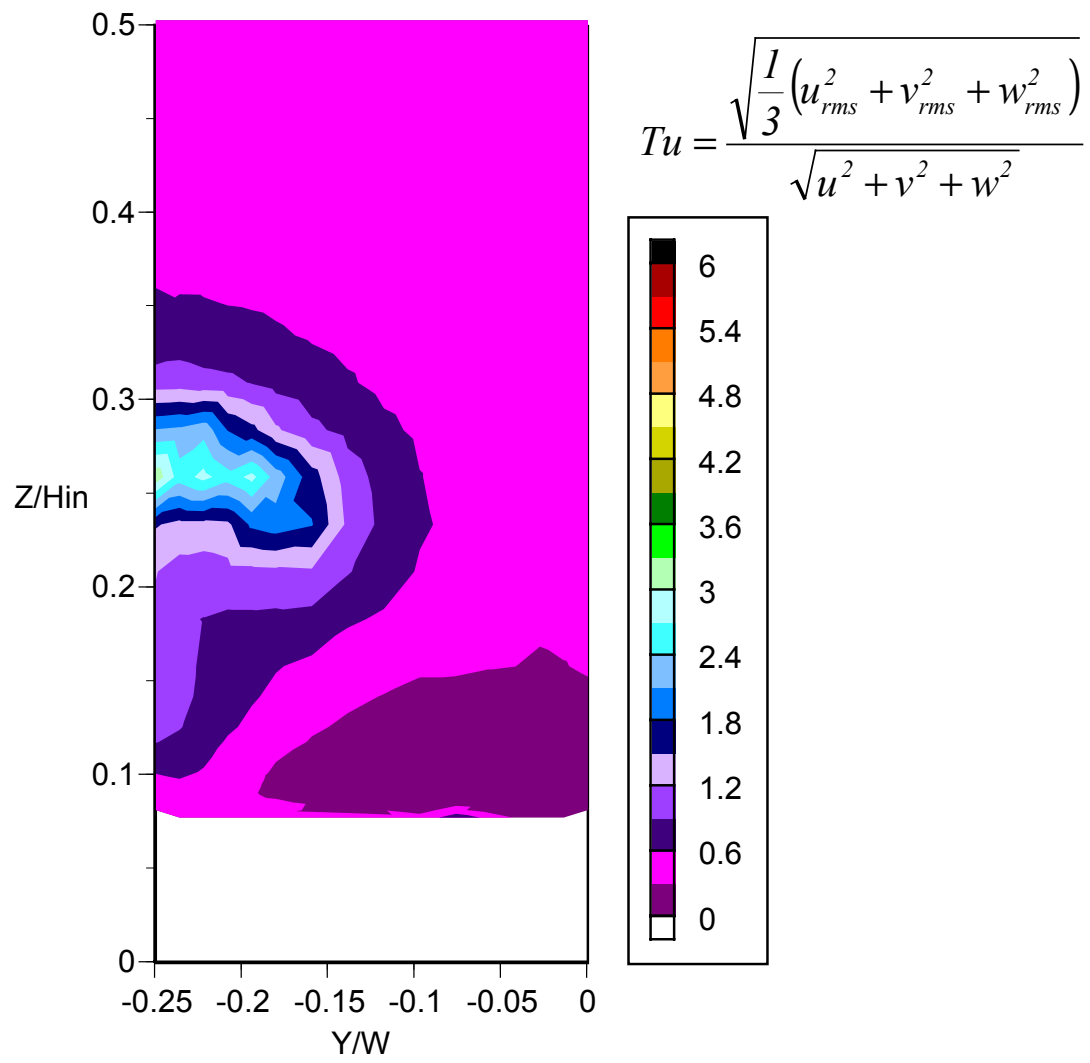

Figure 5.28 Contours of the local turbulence levels, $T u$, within plane $2 p$ (normalized local mass-averaged velocity $u / u_{\text {in }}=2.60$ ). 
Experimental

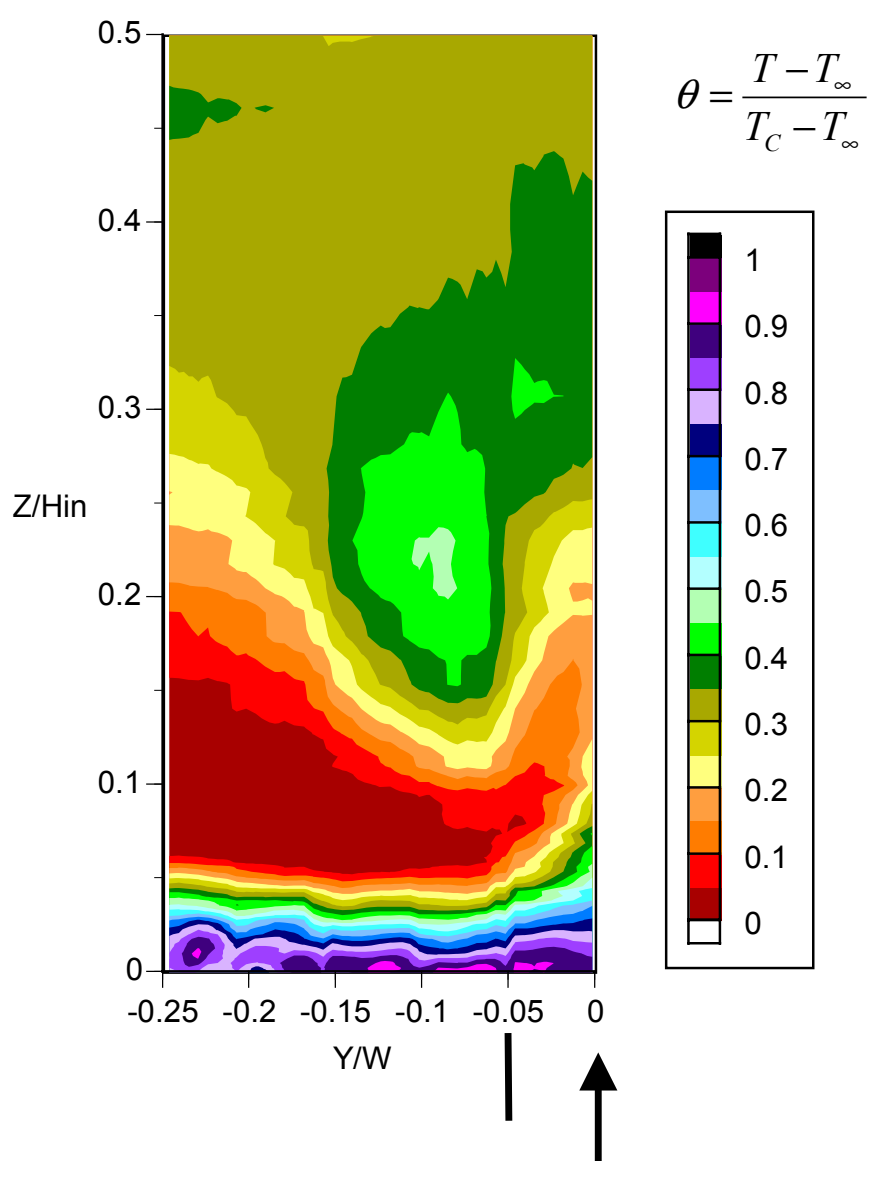

CFD Predictions

(Stitzel, 2001)

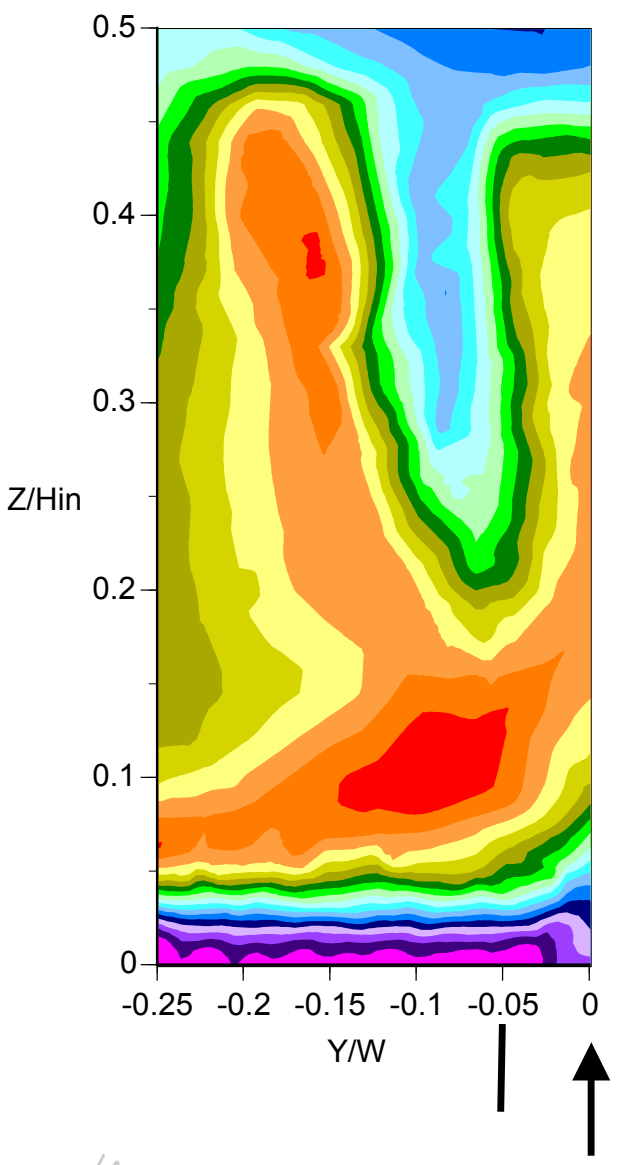

Figure 5.29 A comparison of normalized temperature contours measured experimentally to those predicted computationally in plane $1 \mathrm{p}$. 


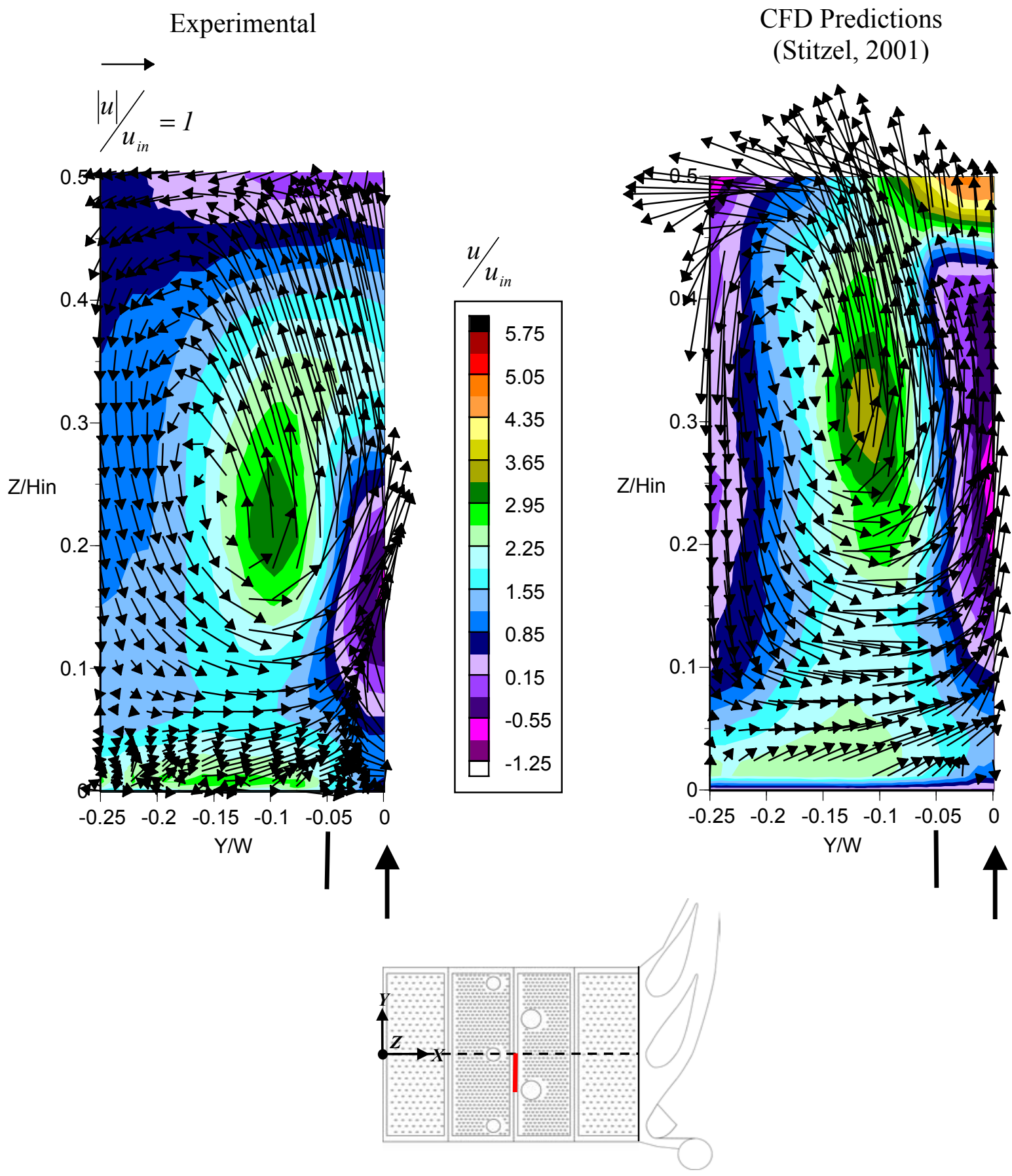

Figure 5.30 Comparison of normalized velocity plots showing contours of $u / u_{i n}$, and vectors of $v / u_{\text {in }}$ and $w / u_{\text {in }}$ for plane $1 \mathrm{p}$. 
Experimental

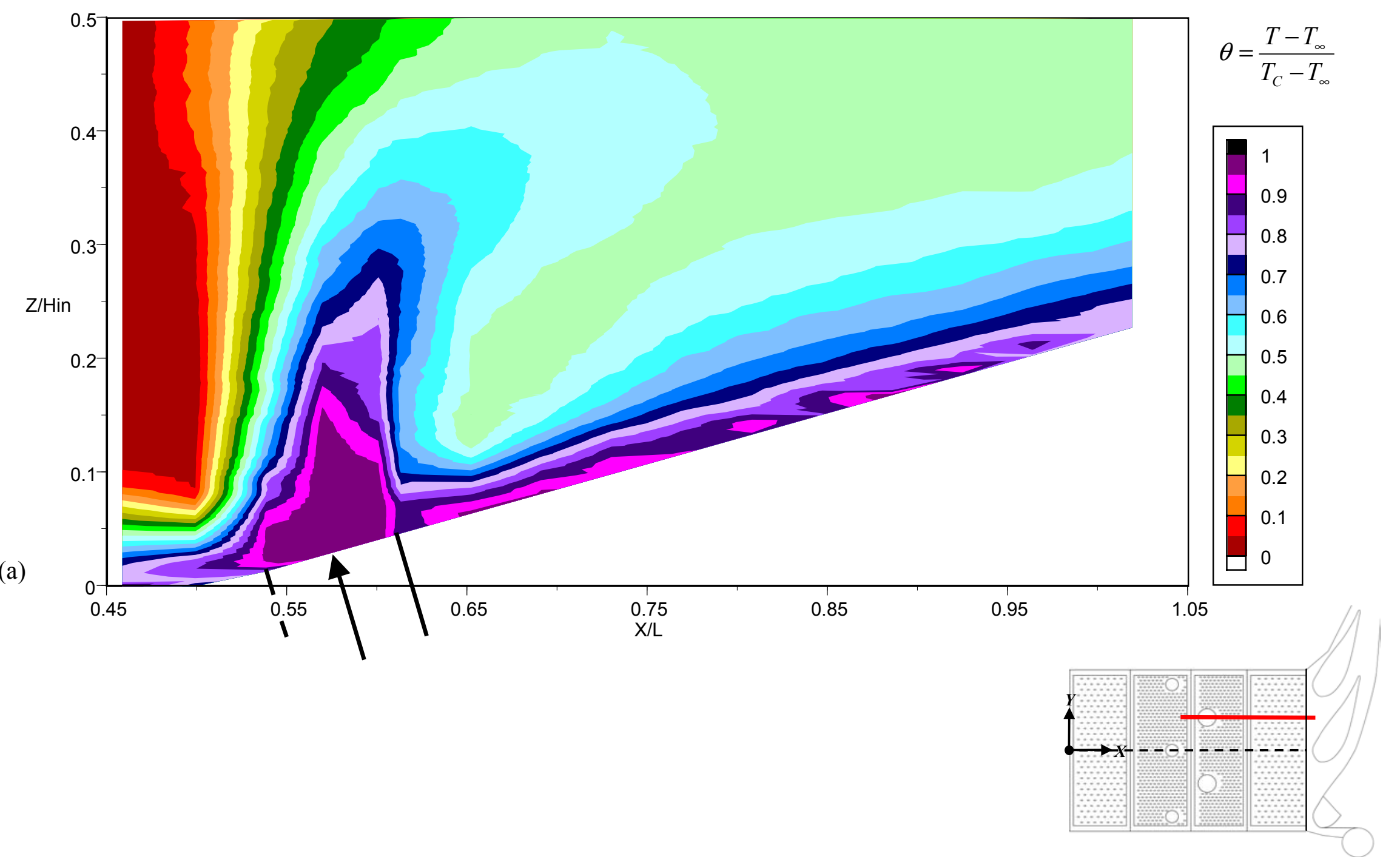

Figure 5.31 Comparison of temperature contours for plane 2s; (a) experimental, (b) computational. 
CFD Predictions (Stitzel, 2001)

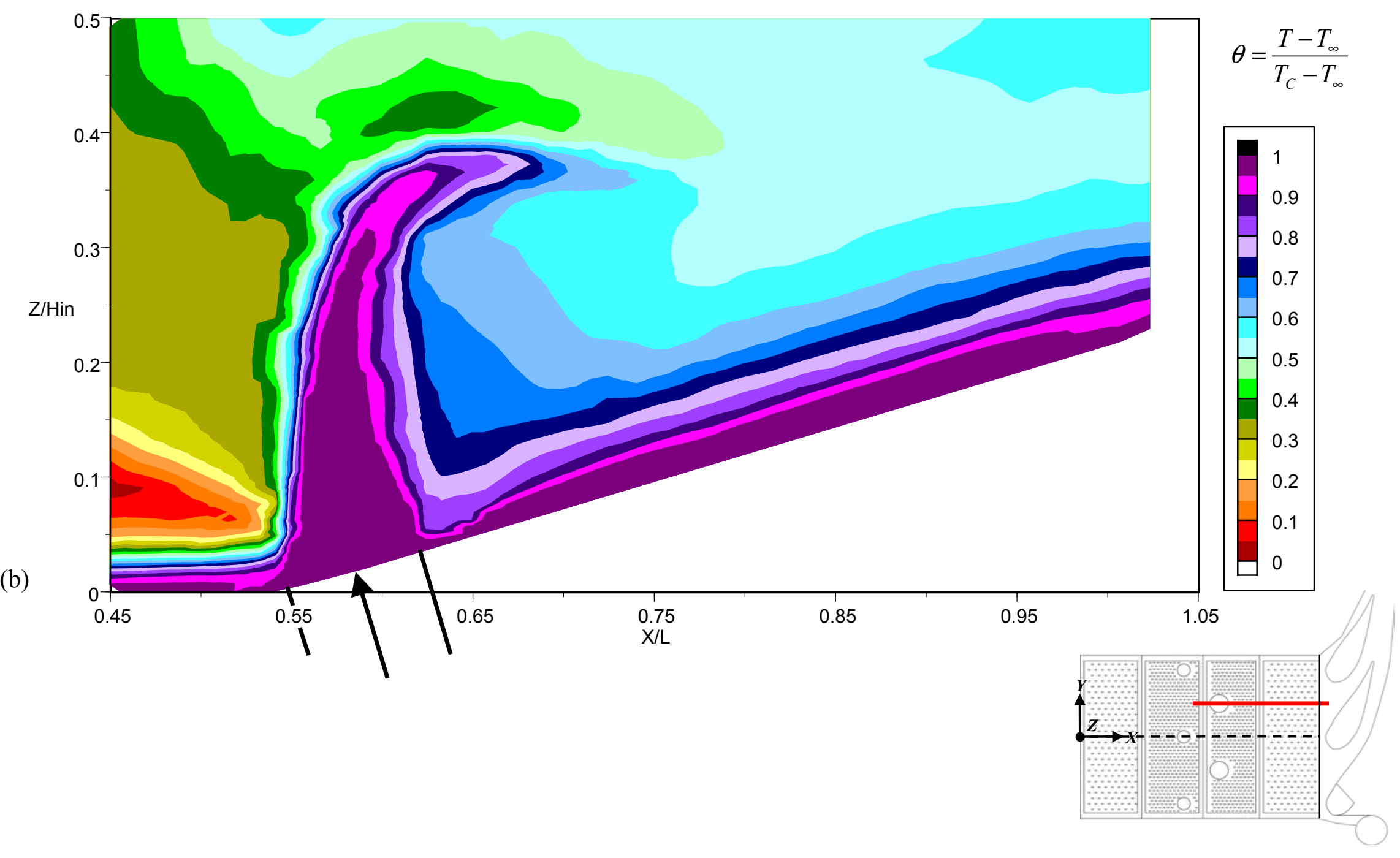

Figure 5.31 Comparison of temperature contours for plane 2s; (a) experimental, (b) computational. 


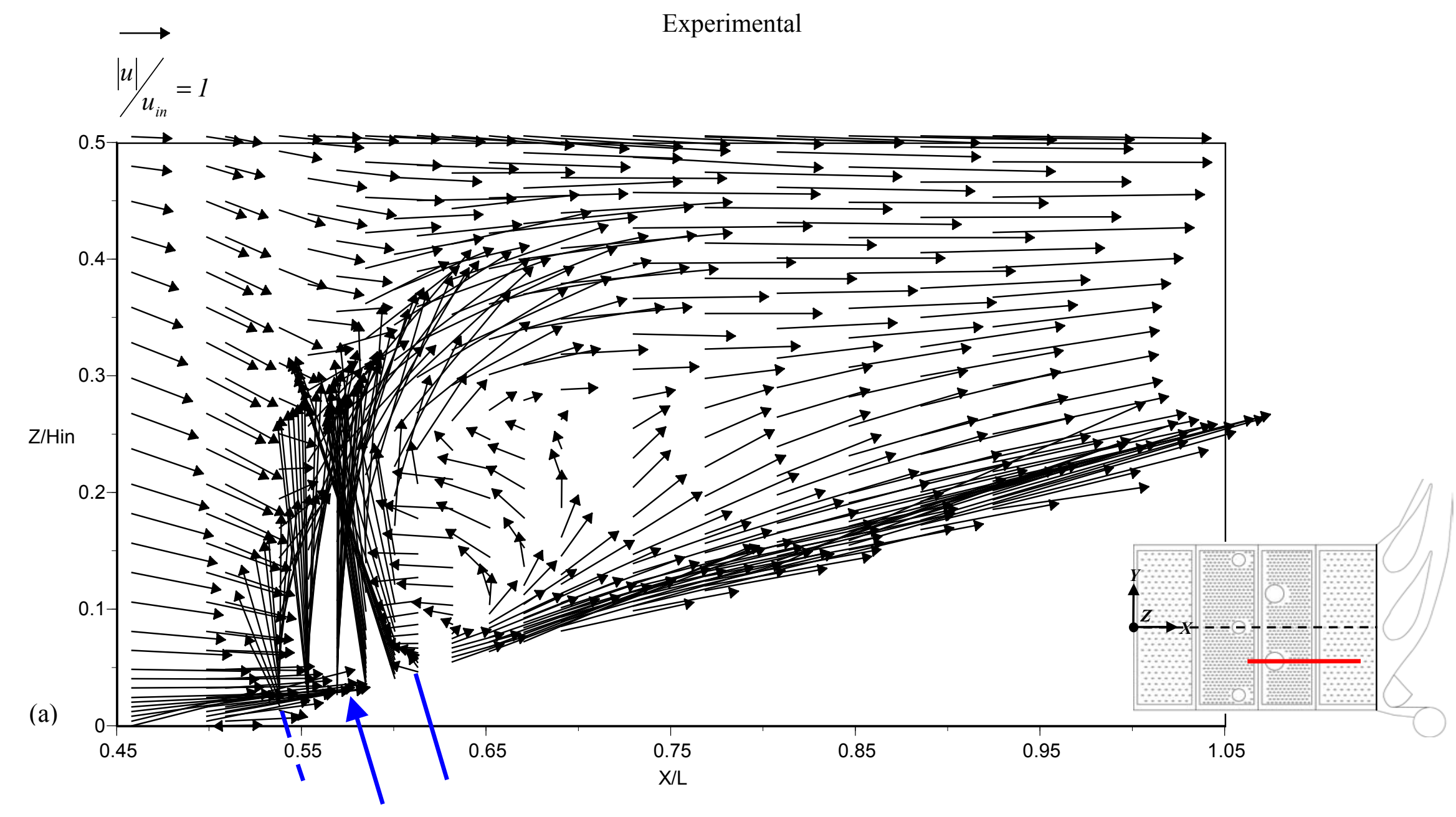

Figure 5.32 Comparison of $u / u_{i n}$ and $w / u_{i n}$ velocity vectors plane $2 \mathrm{~s}$; (a) experimental, (b) computational. 
CFD Predictions (Stitzel, 2001)

$|u| / u_{\text {in }}=1$

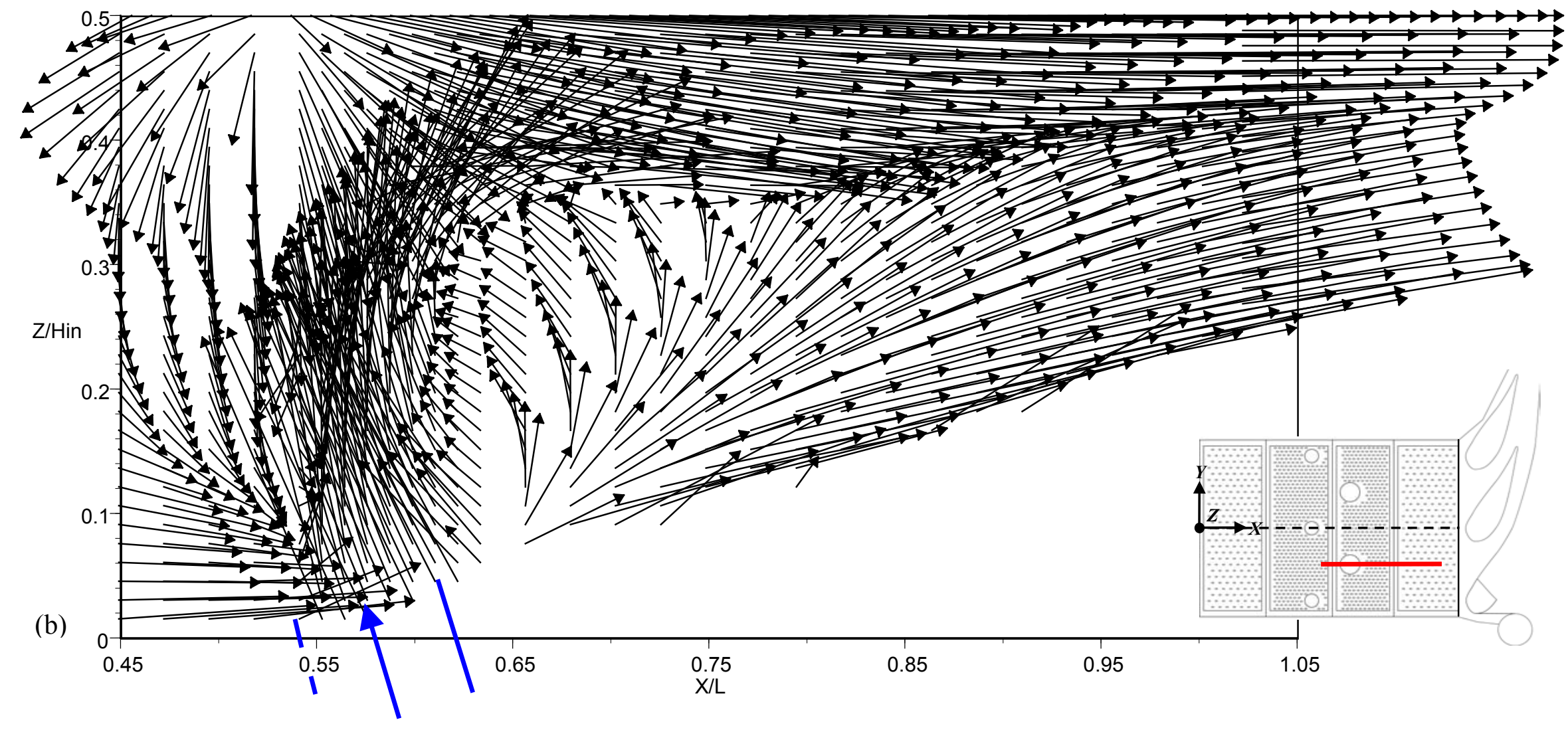

Figure 5.32 Comparison of $u / u_{\text {in }}$ and $w / u_{\text {in }}$ velocity vectors plane $2 \mathrm{~s}$; (a) experimental, (b) computational. 


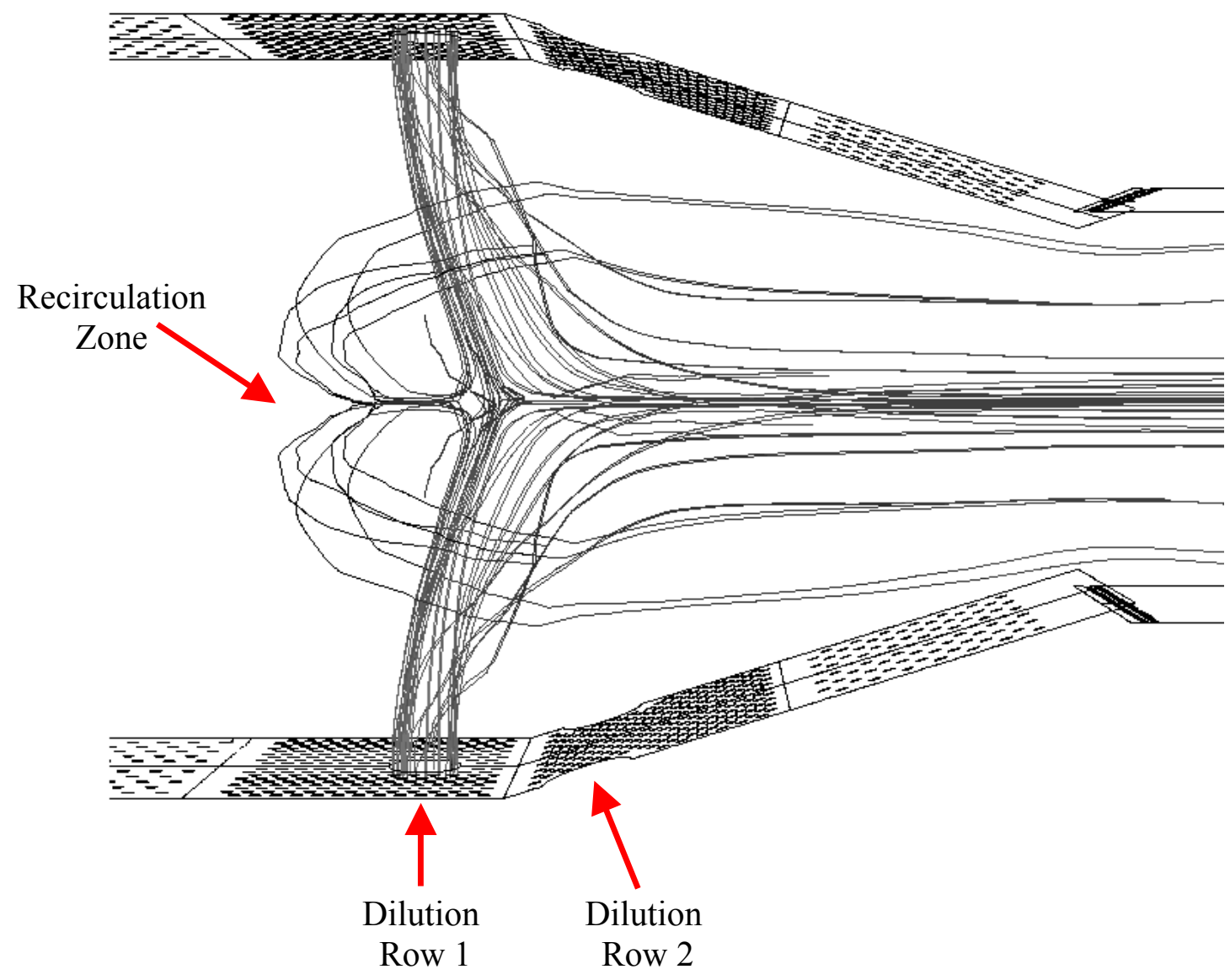

Figure 5.33 Computational predictions of the streamlines from dilution row one (Stitzel, 2001). 
Experimental

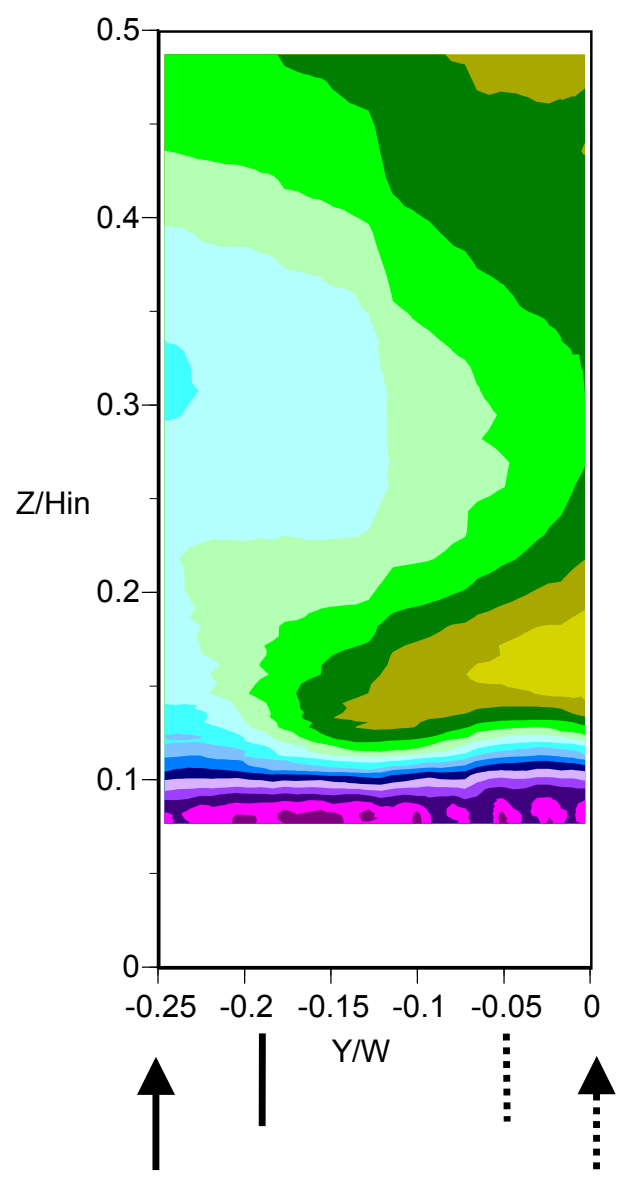

CFD Predictions

(Stitzel, 2001)

$$
\theta=\frac{T-T_{\infty}}{T_{C}-T_{\infty}}
$$
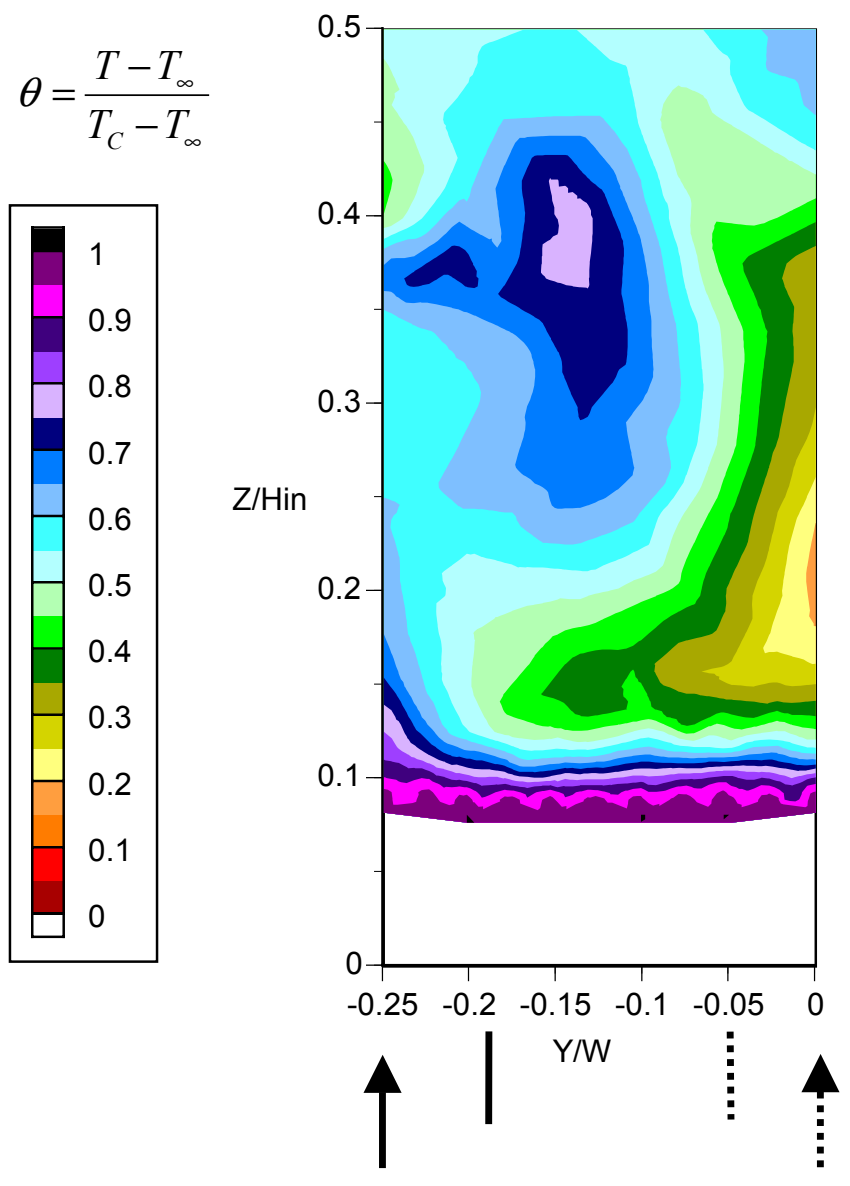

Figure 5.34 Comparison of normalized temperature contours for plane 2p; CFD results on the right, experimental on the left. 


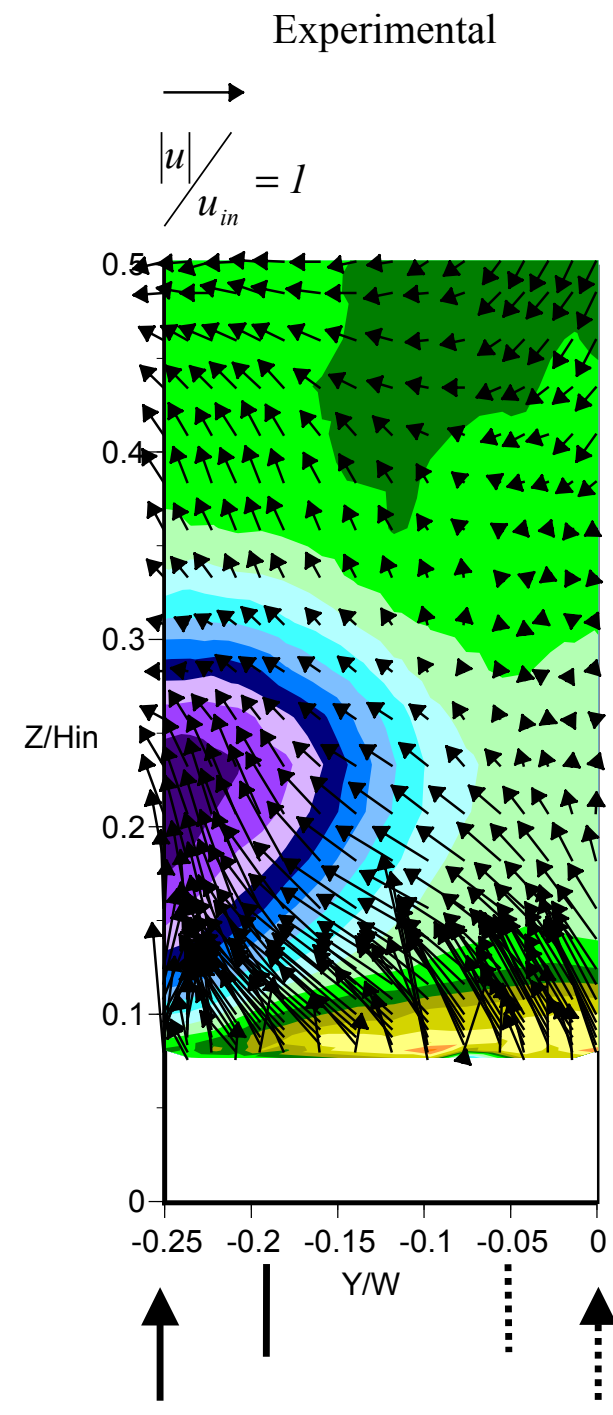

CFD Predictions

(Stitzel, 2001)

$u / u_{\text {in }}$

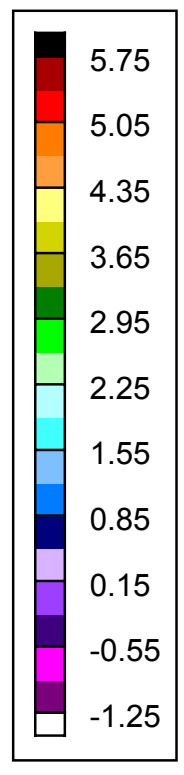

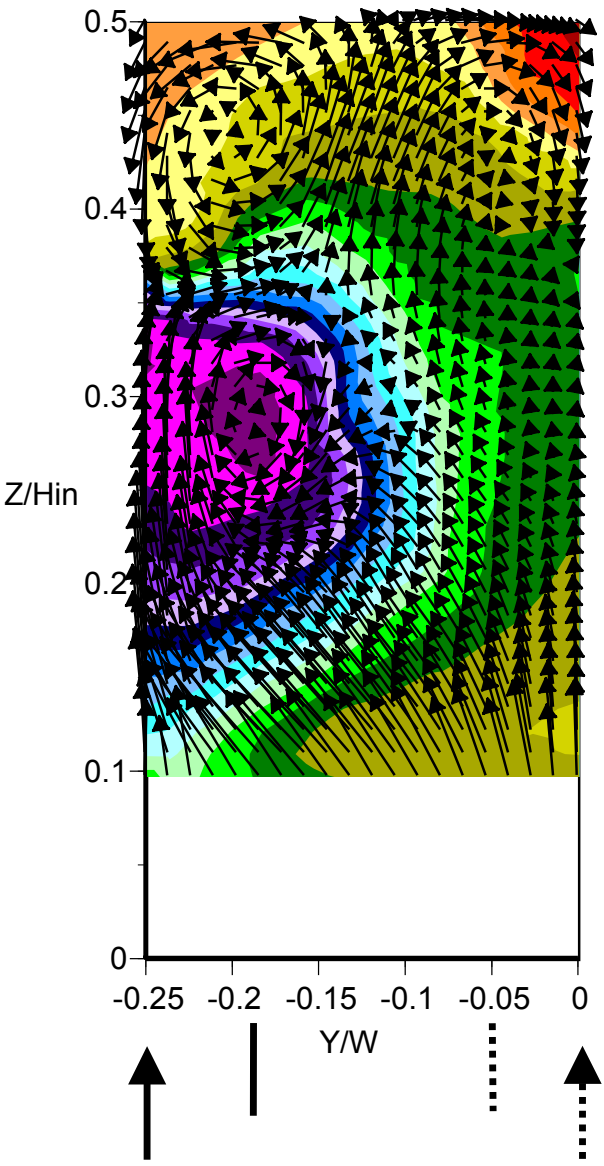

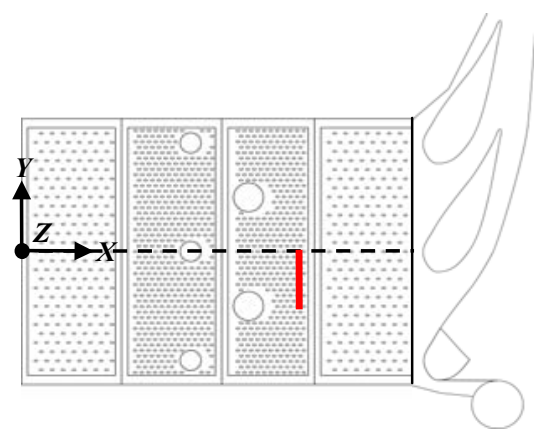

Figure 5.35 Comparison of normalized velocity plots showing contours of $u / u_{i n}$, and vectors of $v / u_{\text {in }}$ and $w / u_{\text {in }}$ for plane $2 \mathrm{p}$; CFD results on the right, experimental on the left. 


\section{Chapter 6: Conclusions and Recommendations for Future Work}

In an effort to provide a better understanding of the thermal and flow mixing phenomena of film-cooling and dilution flows within the combustor of an aero-engine, a database of thermal and flow fields was developed. This data will allow designers to better compare their computational predictions of the behavior of gas turbine combustors, in addition to reducing non-uniformities before entering the turbine vane cascade. An optimized combustor design will help to maximize the effectiveness of the coolant along the turbine vane endwall and prevent premature wear in the combustor and turbine by preventing hot streaks.

The combustor simulator used in this study was a complete linear representation of an aero-engine with the design provided by our sponsor. Upstream of a turbine vane sector, the simulator combined the effects of two rows of streamwise staggered and spanwise aligned dilution jets with film-cooling along the combustor liner walls. Moreover, flow conditions representative of an actual aircraft engine were used when taking the reported measurements. In all, a database documenting the thermal and flow fields within a full three-dimensional, linear combustor simulator was measured. The three-component laser Doppler velocimeter measurements were made to quantify the flow field while a thermocouple rake was used to quantify the thermal field.

This chapter summarizes the major results of this study. The last section concludes with suggestions and recommendations for any future work within this area.

\subsection{Film-Cooling Thermal Field Results}

The first tests conducted were to document the thermal fields downstream of multiple rows of film-cooling jets while determining the effect of tripling the momentum flux out of the combustor liner panels $(I=3$ and 9$)$. Recall that the thermal field measurements for the film-cooling study were made two cooling hole diameters downstream of 15 rows of staggered film-cooling holes. The film-cooling jets closest to the measurement plane (the last row) had their coolest region on the wall with a 
secondary cool region residing slightly further off the wall. This secondary region was a remnant of the upstream row of film-cooling jets that exited from an aligned cooling hole. The adjacent jets in the staggered pattern exhibited only one large core of cooler fluid located off the wall. With only one core of cooler fluid, the data suggests that the two regions had mixed out in a streamwise distance of nine cooling hole diameters. Also apparent was the fact that the core of the jets from the upstream row was further off the wall. As the jets progressed downstream, the momentum flux of the jets resulted in a jet trajectory moving away from the wall.

The major difference between the two film-cooling flow cases considered was in the thickness of the film-cooling layers. The thickness of the film-cooling layer was $28 \%$ thicker for the $I=9$ case as compared with the $I=3$ case. This greater thickness implies that the penetration depth for the $I=9$ case was greater than for the $I=3$ case. Though the film-cooling layer was thicker, the core of the jets remained nominally at the same temperature level for both momentum fluxes. Furthermore, for an $I=9$ the temperatures near the wall and between the jets were slightly cooler as compared to the $I=3$ case.

The effect of increasing the combustor liner momentum flux relative to the interaction with the downstream dilution jets was also noted. Even though the filmcooling was increased, the dilution jet exhibited the same features. Slightly higher temperature levels near the wall were measured for the $I=9$ case relative to the $I=3$ case. No major cooling improvements, however, were experienced along the liner wall for the higher coolant flow case. Directly downstream of the dilution jet, the thermal field contours indicated that the upward motion of the dilution jet entrained the filmcoolant. The core of the kidney-shaped structure containing the counter rotating vortices formed as a result of the jet in crossflow was slightly larger, had a higher penetration depth, and was slightly cooler at the core for the $I=9$ case as compared to the $I=3$ case.

\subsection{Thermal and Flow Field Results for High Momentum Dilution Jets}

A streamwise temperature profile through a first row dilution jet with a high momentum flux ratio $(I=128)$ showed that the jet penetrated to $15 \%$ of the total 
combustor inlet height before the mainstream redirected the jet in the primary flow direction. An overall bulk reduction in the combustor's core temperature of $25 \%$ occurred due to the flow injection from the first row of dilution.

Of particular interest was the film-cooling interaction with the dilution jet. At the leading edge, a relatively thick layer of film-coolant stagnated onto the dilution jet. At the trailing edge, the dilution jet entrained much of the film-coolant, leaving behind a thin film of coolant. Also at the leading edge of the jet, the temperature gradients were quite high at the jet-mainstream interface. Downstream of the dilution jet, the thermal contours spread due to turbulent mixing induced by the dilution flow.

A temperature profile nearly eight film-cooling hole diameters downstream of the trailing edge of the high momentum dilution hole showed a large mushroom shaped temperature profile. This profile resulted from the counter-rotating vortex pair described by others in the open literature for jets in crossflow. Because this temperature profile was taken downstream of two aligned jets, the centers of the counter-rotating vortex pair were separated in the pitchwise direction more than for a single jet in crossflow. No interaction was noted between adjacent dilution holes as a result of the relatively large pitchwise hole spacing (5.4 dilution hole diameters).

The presence of ample film-cooling behind the dilution jet was noted even though only one row of film-cooling jets was present downstream the dilution hole. A horseshoe vortex at the leading edge of the dilution jet had transported film-coolant from upstream to the trailing edge of the dilution jet. Moreover, with increasing pitchwise distance from the core of the jet, the entrainment effects weakened. This was confirmed as the cool cores of the numerous film-cooling jets become increasingly visible with growing distance from the jet centerline. On either side of the dilution jet, no mixing of the hot combustor gasses with the dilution jet or the film-cooling was seen. A measured hot streak of gas passed between the holes in the first row of dilution.

Three-component velocity measurements showed a counter-rotating vortex pair resulting from the shear produced from the dilution jet-mainstream interaction. The centers of the counter-rotating vortex pair were spread relatively far apart due to the opposing dilution jets. The size of the counter-rotating vortex pairs was large as it extended over half the combustor simulator inlet height and $20 \%$ of its width. 
Near the center of the counter-rotating vortex, streamwise velocities over three times that of the inlet velocity were measured. The contours of the streamwise velocity components were also mushroom shaped with the peak values slightly off the core of the vortex. Along the dilution jet centerline, negative streamwise velocities were measured indicating the recirculating region just downstream of the jet. These negative velocities were consistent with the thermal fields, where the film-cooling was entrained by the dilution jet. Streamwise velocities of zero at the midspan indicated a stagnation region, which was created due to the impact region of the top and bottom dilution jets.

Contour plots of the turbulence levels, $T L$, indicated that the largest levels occurred slightly off of the counter-rotating vortex center where the largest streamwise velocity components existed and also occurred in the jet impact region. The largest local turbulence, $T u$, was found at the midspan along the dilution jet centerline where the impact region dominated and the flow was nearly stagnant. In general, turbulence levels were quite high at an average of $24 \%$ over most of the combustor width just downstream of this first row of dilution jets.

\subsection{Thermal and Flow Field Results for Low Momentum Dilution Jets}

Injected at a $15.8^{\circ}$ angle from the vertical axis, the staggered second row of dilution holes were approximately 1.4 times larger in diameter than the first row. The momentum flux for the second row of dilution jets was significantly smaller than the first at $I=32$. The streamwise thermal contours indicated a sudden change in trajectory of the dilution jet core from initially towards the upstream and then re-directed towards the downstream, after the jet exited into the mainstream. When comparing the respective penetration heights between the first and second rows of dilution, the first row penetrated to a 1.2 hole diameters, whereas the second row penetrated to roughly the same distance at 1.1 hole diameters.

Slightly before the leading edge of the second row dilution jet, the streamwise thermal contours indicated that spreading of the first row dilution jets had not yet diluted the mainstream flow at this pitch location. At the trailing edge of the jet, the presence of 
film-cooling was indicative of a possible horse-shoe vortex wrapping the coolant around the dilution jet. The thermal field results showed a large recirculating region where much of the film-cooling was entrained and carried away by the dilution jet leaving behind a thinner film of coolant downstream of the injection. Proceeding downstream of the second dilution row, the film thickness grew substantially due to the high turbulence levels produced by the dilution jets.

A pitchwise thermal field downstream of the second row dilution depicted the first and second rows of dilution. At this point, two warmer regions behind the first row dilution jet are visible. It was likely that these warm regions near midspan and the wall were the result of unmixed warmer fluid flowing around the stagnation region, and the warmer fluid trapped behind recirculating region. Adding to the overwhelming lack of uniformity at this point within the combustor was the fact that the core of the second row dilution jet was almost twice as cold as the first row. With only $31 \%$ of the combustor's length left and no more rows of dilution, a streaky, non-uniform temperature profile at the combustor exit was highly likely.

Streamwise vectors along the second row dilution jet centerline, showed that the flow was angled downward as it impacted the leading edge of the jet. This behavior resulted from the flow being deflected under the stagnation region created at the midspan by the first row of dilution jets. Downstream of the jet ejection, the vectors clearly illustrated the separated, recirculating region where again the film-coolant flow was entrained by the shear forces created by the dilution jet.

Downstream of the second row of dilution holes, the flow was continuously accelerated along the combustor surface due to the contraction. Specifically, the flow field vectors showed an acceleration in the near wall region due to added film-cooling flow, and above the jet injection at the mid-span due to the flow bypassing the dilution jet blockage. In general, as the flow was accelerated, the velocity profiles become more uniform across the combustor height towards the exit. Traces of the faster film-cooling and midspan regions, however, were still visible in the streamwise velocity profiles at the combustor exit.

Large values of the local turbulence levels were found throughout the dilution jet especially at the leading and trailing edges and at the film-cooling hole exits. At the 
leading and trailing edges of the jet, the largest shear layers existed due to the interaction of the jet with the mainstream and the large recirculation region. The average local turbulence level near the combustor simulator exit was $24 \%$.

Three component velocity measurements, taken one dilution hole diameter downstream of the trailing edge of a second row dilution hole, hardly resemble those behind the first row of dilution jets. A strong kidney vortex was not seen at this location. Slight traces of it were found near the combustor mid-span, however it is most likely that the high turbulence levels have caused considerable reductions in the swirl velocities. One major characteristic that remained was the streamwise recirculating region behind the dilution jet.

\subsection{Comparisons of Experimental Measurements to Computational Predictions}

Comparisons of the data experimentally measured to that predicted computationally showed similarities, as well as a number of dissimilarities. Predicted flow field data indicated the characteristic counter-rotating vortices and the entrainment behind the dilution jet injection. However, temperature predictions of the first row of dilution jets exhibited a cold spike of fluid rather than the larger kidney-shaped temperature profile measured experimentally. At the midspan where the first row dilution jets impact one another, computational predictions indicated relatively large streamwise velocities while experimental measurements showed the same velocity to be nearly zero.

The computational results generally appeared much less diffuse than the experimental data, which showed an underprediction in the mixing of the flow, especially in the impact region of the first row of dilution jets. This under-prediction in mixing was due to an underprediction in turbulence. For example, the thickness in the film-coolant behind the second row of dilution jets was much greater for experimental measurements than for the CFD predictions. Another byproduct of this underprediction of turbulence was that the penetration of the dilution jets was higher for the CFD. The jets appeared more coherent, while creating an especially large recirculation zone at the impact of the 
first row dilution jets. Downstream of the second row of dilution jets, computational results showed three vortices. These vortices were not seen in the experimental results, which was most likely due to the high turbulence levels having mixed out the flow. It is important to note that strong jets in crossflow are difficult to predict and although CFD predictions showed similarities to measured data, the need for experimental verification is evident from these comparisons.

\subsection{Recommendations for Future Work}

Based of the results of this research, the following recommendations are made for future research within this area. Additional flow field measurements should be made at the leading edge of the dilution jets to possibly document the presence and effects of a horse-shoe vortex. It would be interesting to create a streamwise progression of flow and thermal measurements across the pitch downstream of the dilution jet. This would be useful in tracking the progression of the counter-rotating vortex pair and determining more about its mixing characteristics through the combustor. Another suggestion is to change the momentum flux of the dilution jets in an effort to find the optimum combination for thorough mixing and uniform combustor exit profiles in both velocity and temperature. 


\section{References}

Ahmed, S. A., 1998, "Velocity Measurements and Turbulence Statistics of a Confined Isothermal Swirling Flow," Experimental Thermal and Fluid Science, vol. 17, pp. 256-264.

Ames, F. E., and Moffat, R. J., 1990, "Effects of Simulated Combustor Turbulence on Boundary Layer Heat Transfer," Heat Transfer in Turbulent Flow 1990: AIAA/ASME Thermophysics and Heat Transfer Conference, Seattle Washington, June 18-20, 1990.

Anacleto, P., Heitor, M. V., and Moreira, A. L. N., 1996, "The Mean and Turbulent Flowfields in a Model RQL Gas-Turbine Combustor," Experiments in Fluids, vol. 22, pp. 153-164.

Barringer, M. D., 2001, "Design and benchmarking of a Combustor Simulator Relevant to Gas Turbine Engines," Master's Thesis, Department of Mechanical Engineering, Virginia Polytechnic Institute and State University.

Barringer, M. D., Richard, O. T., Walter, J. P., Stitzel, S. M., and Thole, K. A., 2002, "Flow Field Simulations of a Gas Turbine Combustor," Journal of Turbomachinery, vol. 124, pp. 508-516.

Bicen, A. F., Tse, D., and Whitelaw, J. H., 1988, "Flow and Combustion Characteristics of an Annular Combustor," Combustion and Flame, vol. 72, pp. 175-192.

Blomeyer, M., Krautkremer, B., Hennecke, D. K., and Doerr, T., 1999, "Mixing Zone Optimization of a Rich-Burn/Quick-Mix/Lean-Burn Combustor," Journal of Propulsion and Power, vol. 15, no. 2, pp. 288-295. 
Busnaina, A. A., 1987, "Lateral Jet Injection into Isothermal Combustor Flowfields," Chemical Engineering Communications, vol. 54 (1-6), pp. 265-278.

Cameron, C. D., Brouwer, J., Wood, C. P., and Samuelson, G. S., 1989, “A Detailed Characterization of the Velocity and Thermal Fields in a Model Can Combustor with Wall Injection," Journal of Engineering for Gas Turbines and Power, vol. 111, pp. 31-35.

Colban, W. F., 2001, "Effects of Realistic Combustor Exit Profiles on a Turbine Vane Endwall," Master's Thesis, Department of Mechanical Engineering, Virginia Polytechnic Institute and State University.

Crocker, D. S., Smith, C. E., and Myers, G. D., 1994, "Pattern Factor Reduction in a Reverse Flow Gas Turbine Combustor Using Angled Dilution Jets," ASME Paper No. 94-GT-406.

Doerr, T., Blomeyer, M., and Hennecke, D. K., 1997, “Optimization of Multiple Jets Mixing With a Confined Crossflow," Journal of Engineering for Gas Turbines and Power, vol. 119, pp. 315-321.

Ebbinghaus, A., and Swithenbank, J., 1995, "Mixing of Multiple Jets in a Can Combustor," ASME Paper No. 95-GT-257.

Goebel, S. G., Abauf, N., Lovett, J. A., and Lee, C. P., 1993, "Measurements of Combustor Velocity and Turbulence Profiles," ASME Paper No. 93-GT-228.

Goldstein, R. J., Lau, K. Y., and Leung, C. C., 1983, "Velocity and Turbulence Measurements in Combustion Systems," Experiments in Fluids, vol. 1, pp. 93-99. 
Gritsch, M., Martiny, M., Schulz, A., Kim, S., and Wittig, S., 1998, “Gas Turbine Heat Transfer: Newest Developments in Component Performance," Heat Transfer 1998: Proceedings of the $11^{\text {th }}$ International Heat Transfer Conference (IHTC), Kyongju, Korea, August 23-28, 1998.

Halls, B. A, 1970, “Nozzle Guide Vane Cooling,” AGARD, Cp No. 73, Paper 25.

Holdeman, J. D., 1993, "Mixing of Multiple Jets with a Confined Subsonic Crossflow," Prog. Energy Combust. Sci., vol. 19, pp. 31-70.

Koutmos, P., and McGuirk, J. J., 1989, "Isothermal Flow in a Gas Turbine Combustor A Benchmark Experimental Study," Experiments in Fluids, vol. 7, pp. 344-354.

Lawson, R. J., 1993, “Computational Modeling of an Aircraft Engine Combustor to Achieve Target Exit Temperature Profiles,” ASME Paper No. 93-GT-164.

Lefebvre, A. H., 1999, Gas Turbine Combustion (Philadelphia, PA.: Taylor \& Francis).

Liou, T. M., and Wu, Y. Y., 1992, "LDV Measurements of the Flowfield in a Simulated Combustor with Axial and Side Inlets," Experimental Thermal and Fluid Science, vol. 5, pp. 401-409.

Malecki, R. E., Rhie, C. M., McKinney, R. G., Ouyang, H., Syed, S. A., Colket, M. B., and Madabhushi, R. K., 2001, "Application of an Advanced CFD-Based Analysis System to the PW6000 Combustor to Optimize Exit Temperature Distribution Part I: Description and Validation of the Analysis Tool," ASME Paper No. 2001GT-0062.

Moffat, J. R., 1988, "Describing the Uncertainties in Experimental Results," Experimental Thermal and Fluid Science, vol. 1, pp. 3-17. 
Moss, R. W., 1992, “The Effects of Turbulence Length Scale on Heat Transfer,” Ph. D. Dissertation, Department of Engineering Science, University of Oxford, Report No. OUEL 1924/92.

National Instruments, 2002, http://www.ni.com (Austin, TX.: National Instruments Corporation)

Pietrzyk, J. R., 1989, “Experimental Study of the Interaction of Dense jets with a Crossflow for Gas Turbine Applications,” Ph. D. Dissertation, Department of Mechanical Engineering, University of Texas at Austin.

Pratt \& Whitney, 1988, The Aircraft Gas Turbine Engine and Its Operation, $3^{\text {rd }}$ Edition, (East Hartford, CT.: United Technologies Corporation).

Radomsky, R. W., "High Freestream Turbulence Studies on a Scaled-Up Stator Vane,” Ph. D. Dissertation, Department of Mechanical Engineering, University of Wisconsin at Madison.

Richards, C. D., and Samuelsen, G. S., 1992, “The Role of Primary Jets in the Dome Region Aerodynamics of a Model Can Combustor," Journal of Engineering for Gas Turbines and Power, vol. 114, pp. 20-26.

Rolls-Royce, 1992, The Jet Engine (Derby, England: The Technical Publications Department, Rolls-Royce plc.).

Rydholm, H. A., 1998, “An Experimental Investigation of the Velocity and Temperature Fields of Cold Jets Injected Into a Hot Crossflow," Journal of Turbomachinery, vol. 120 , pp. 320-326.

Sgarzi, O., and Leboeuf, F., 1997, "Analysis of Vortices in Three-Dimensional Jets Introduced in a Cross-Flow Boundary-Layer,” ASME Paper No. 97-GT-517. 
Sinha, A. K., Bogard, D. G., and Crawford, M. E., 1990, "Gas Turbine Film-Cooling: Flowfield Due to a Second Row of Holes," ASME Paper No. 90-GT-44.

Snyder, T. S., Stewart, J. F., Stoner, M. D., and McKinney, R. G., “Application of an Advanced CFD-Based Analysis System to the PW6000 Combustor to Optimize Exit Temperature Distribution - Part II: Comparison of Predictions to Full Annular Rig Test Data,” ASME Paper No. 2001-GT-0064.

Stevens, S. J., and Carotte, J. F., 1990, "Experimental Studies of Combustor Dilution Zone Aerodynamics, Part I: Mean Flowfields," Journal of Propulsion, vol. 6, no. 3, pp. 297-304.

Stitzel, S. M., 2001, "Flow Field Computations of Combustor-Turbine Interaction in a Gas Turbine Engine,” Master's Thesis, Department of Mechanical Engineering, Virginia Polytechnic Institute and State University.

TSI, 1995, LDV/APV System Installation Manual (St. Paul, MN.: TSI Incorporated).

TSI, 1999, Information was provided to a previous student through personal communication.

TSI, 2000, FIND for Windows (Version 1.4) - Instruction Manual (St. Paul, MN.: TSI Incorporated).

Van Fossen, G. J., and Bunker R. S., 2000, “Augmentation of Stagnation Region Heat Transfer Due to Turbulence From a DLN Can Combustor," Journal of Turbomachinery, vol. 123, no. 1, pp. 140-146.

Van Fossen, G. J., and Bunker R. S., 2002, "Augmentation of Stagnation Region Heat Transfer Due to Turbulence From an Advanced Dual-Annular Combustor," ASME Paper No. GT-2002-30184. 
Wilson, D. G., and Korakianitis, T., 1998, The Design of High-Efficiency

Turbomachinery and Gas Turbines (Upper Saddle River, NJ.: Prentice-Hall, Inc.). 


\section{Appendix A: Characteristics of Pressure Transducers}

This appendix documents the all of the available pressure transducers, some of which were used in setting the flow conditions for the experiments conducted in this study. A complete listing of transducer models, serial numbers, coefficients, and nonlinearity is given. Note that the coefficients shown here are calculated according to the original manufacturer's calibration data. They are subject to change over time, particularly the zero offset coefficient, and should always be checked before conducting any experiments to ensure accurate readings. 
Table A.1 Pressure Transducer Specifications; Omega Serial Number: 60518167

General:

\begin{tabular}{|c|c|}
\hline Manufacturer & Omega \\
\hline Model Number & PX653 \\
\hline Serial Number & 60518167 \\
\hline Range & $0-0.50^{\prime \prime} \mathrm{H}_{2} \mathrm{O}$ \\
\hline
\end{tabular}

Calibration Data:

\begin{tabular}{|c|c|}
\hline Applied Pressure (in- $\left.\mathbf{H}_{2} \mathbf{O}\right)$ & Transducer Output \\
\hline 0.000 & 0.999 \\
\hline 0.125 & 2.001 \\
\hline 0.250 & 2.999 \\
\hline 0.375 & 3.998 \\
\hline 0.500 & 5.000 \\
\hline
\end{tabular}

\begin{tabular}{|c|c|}
\hline Slope Coefficient (in- $\left.\mathbf{H}_{\mathbf{2}} \mathbf{O} / \mathbf{V}_{\text {DC }}\right)$ & 0.125 \\
\hline Offset Coefficient (in- $\left.\mathbf{H}_{\mathbf{2}} \mathbf{O}\right)$ & -0.125 \\
\hline Non-Linearity & $\pm 0.25 \%$ Full-Scale \\
\hline
\end{tabular}


Table A.2 Pressure Transducer Specifications; Omega Serial Number: 60518721

General:

\begin{tabular}{|c|c|}
\hline Manufacturer & Omega \\
\hline Model Number & PX653 \\
\hline Serial Number & 60518721 \\
\hline Range & $0-2.0^{\prime \prime}$ \\
\hline
\end{tabular}

Calibration Data:

\begin{tabular}{|c|c|}
\hline Applied Pressure (in- $\left.\mathbf{H}_{2} \mathbf{O}\right)$ & Transducer Output \\
\hline 0.000 & 0.999 \\
\hline 0.500 & 2.001 \\
\hline 1.000 & 2.999 \\
\hline 1.500 & 3.998 \\
\hline 2.000 & 5.000 \\
\hline
\end{tabular}

\begin{tabular}{|c|c|}
\hline Slope Coefficient (in- $\left.\mathbf{H}_{\mathbf{2}} \mathbf{O} / \mathbf{V}_{\text {DC }}\right)$ & 0.500 \\
\hline Offset Coefficient (in- $\left.\mathbf{H}_{\mathbf{2}} \mathbf{O}\right)$ & -0.500 \\
\hline Non-Linearity & $\pm 0.25 \%$ Full-Scale \\
\hline
\end{tabular}


Table A.3 Pressure Transducer Specifications; Setra Serial Number: 1083663

General:

\begin{tabular}{|c|c|}
\hline Manufacturer & Setra \\
\hline Model Number & 264 \\
\hline Serial Number & 1083663 \\
\hline Range & $0-0.50^{\prime \prime} \mathrm{H}_{2} \mathrm{O}$ \\
\hline
\end{tabular}

Calibration Data:

\begin{tabular}{|c|c|}
\hline Applied Pressure (in- $\left.\mathbf{H}_{2} \mathbf{O}\right)$ & Transducer Output \\
\hline 0.0023 & 0.0769 \\
\hline 0.0523 & 0.5668 \\
\hline 0.1021 & 1.0652 \\
\hline 0.1521 & 1.5639 \\
\hline 0.2022 & 2.0640 \\
\hline 0.2512 & 2.5579 \\
\hline 0.3020 & 3.0703 \\
\hline 0.3507 & 3.5567 \\
\hline 0.4022 & 4.0711 \\
\hline 0.4524 & 4.5718 \\
\hline 0.5020 & 5.0676 \\
\hline
\end{tabular}

\begin{tabular}{|c|c|}
\hline Slope Coefficient (in- $\left.\mathbf{H}_{\mathbf{2}} \mathbf{O} / \mathbf{V}_{\text {DC }}\right)$ & 0.1000 \\
\hline Offset Coefficient (in- $\left.\mathbf{H}_{2} \mathbf{O}\right)$ & -0.0046 \\
\hline Non-Linearity & $\pm 0.206 \%$ Full-Scale \\
\hline
\end{tabular}


Table A.4 Pressure Transducer Specifications; Setra Serial Number: 1083664

General:

\begin{tabular}{|c|c|}
\hline Manufacturer & Setra \\
\hline Model Number & 264 \\
\hline Serial Number & 1083664 \\
\hline Range & $0-0.50 " \mathrm{H}_{2} \mathrm{O}$ \\
\hline
\end{tabular}

Calibration Data:

\begin{tabular}{|c|c|}
\hline Applied Pressure (in- $\left.\mathbf{H}_{\mathbf{2}} \mathbf{O}\right)$ & Transducer Output \\
\hline 0.0020 & 0.0688 \\
\hline 0.0522 & 0.5670 \\
\hline 0.1023 & 1.0672 \\
\hline 0.1523 & 1.5703 \\
\hline 0.2023 & 2.0752 \\
\hline 0.2522 & 2.5791 \\
\hline 0.3025 & 3.0877 \\
\hline 0.3522 & 3.5886 \\
\hline 0.4019 & 4.0842 \\
\hline 0.4522 & 4.5850 \\
\hline 0.5023 & 5.0795 \\
\hline
\end{tabular}

\begin{tabular}{|c|c|}
\hline Slope Coefficient (in- $\left.\mathbf{H}_{\mathbf{2}} \mathbf{O} / \mathbf{V}_{\mathbf{D C}}\right)$ & 0.0996 \\
\hline Offset Coefficient (in- $\left.\mathbf{H}_{\mathbf{2}} \mathbf{O}\right)$ & -0.0045 \\
\hline Non-Linearity & $\pm 0.206 \%$ Full-Scale \\
\hline
\end{tabular}


Table A.5 Pressure Transducer Specifications; Setra Serial Number: 1185966

General:

\begin{tabular}{|c|c|}
\hline Manufacturer & Setra \\
\hline Model Number & 264 \\
\hline Serial Number & 1185966 \\
\hline Range & $0-5.0^{\prime \prime} \mathrm{H}_{2} \mathrm{O}$ \\
\hline
\end{tabular}

Calibration Data:

\begin{tabular}{|c|c|}
\hline Applied Pressure (in- $\left.\mathbf{H}_{\mathbf{2}} \mathbf{O}\right)$ & Transducer Output \\
\hline 0.0190 & 0.0480 \\
\hline 0.4959 & 0.5215 \\
\hline 1.0124 & 1.0402 \\
\hline 1.5141 & 1.5447 \\
\hline 2.0124 & 2.0428 \\
\hline 2.5101 & 2.5436 \\
\hline 3.0238 & 3.0597 \\
\hline 3.5686 & 3.6057 \\
\hline 4.0017 & 4.0364 \\
\hline 4.5033 & 4.5358 \\
\hline 5.0186 & 5.0473 \\
\hline
\end{tabular}

\begin{tabular}{|c|c|}
\hline Slope Coefficient (in- $\left.\mathbf{H}_{\mathbf{2}} \mathbf{O} / \mathbf{V}_{\text {DC }}\right)$ & 0.9988 \\
\hline Offset Coefficient (in- $\left.\mathbf{H}_{2} \mathbf{O}\right)$ & -0.0284 \\
\hline Non-Linearity & $\pm 0.206 \%$ Full-Scale \\
\hline
\end{tabular}


Table A.6 Pressure Transducer Specifications; Setra Serial Number: 1185967

General:

\begin{tabular}{|c|c|}
\hline Manufacturer & Setra \\
\hline Model Number & 264 \\
\hline Serial Number & 1185967 \\
\hline Range & $0-5.0^{\prime \prime} \mathrm{H}_{2} \mathrm{O}$ \\
\hline
\end{tabular}

Calibration Data:

\begin{tabular}{|c|c|}
\hline Applied Pressure (in- $\left.\mathbf{H}_{\mathbf{2}} \mathbf{O}\right)$ & Transducer Output \\
\hline 0.0246 & 0.0722 \\
\hline 0.5237 & 0.5660 \\
\hline 1.0233 & 1.0649 \\
\hline 1.5183 & 1.5617 \\
\hline 2.0205 & 2.0658 \\
\hline 2.5217 & 2.5693 \\
\hline 3.0212 & 3.0710 \\
\hline 3.5099 & 3.5610 \\
\hline 4.0209 & 4.0763 \\
\hline 4.4858 & 4.5410 \\
\hline 5.0213 & 5.0746 \\
\hline
\end{tabular}

\begin{tabular}{|c|c|}
\hline Slope Coefficient (in- $\left.\mathbf{H}_{\mathbf{2}} \mathbf{O} / \mathbf{V}_{\mathbf{D C}}\right)$ & 0.9974 \\
\hline Offset Coefficient (in- $\left.\mathbf{H}_{\mathbf{2}} \mathbf{O}\right)$ & -0.0418 \\
\hline Non-Linearity & $\pm 0.206 \%$ Full-Scale \\
\hline
\end{tabular}


Table A.7 Pressure Transducer Specifications; Setra Serial Number: 1258460

General:

\begin{tabular}{|c|c|}
\hline Manufacturer & Setra \\
\hline Model Number & 264 \\
\hline Serial Number & 1258460 \\
\hline Range & $0-0.25^{\prime \prime} \mathrm{H}_{2} \mathrm{O}$ \\
\hline
\end{tabular}

Calibration Data:

\begin{tabular}{|c|c|}
\hline Applied Pressure (in- $\left.\mathbf{H}_{\mathbf{2}} \mathbf{O}\right)$ & Transducer Output \\
\hline 0.0011 & 0.0835 \\
\hline 0.0255 & 0.5596 \\
\hline 0.0512 & 1.0728 \\
\hline 0.0760 & 1.5736 \\
\hline 0.1012 & 2.0668 \\
\hline 0.1259 & 2.5825 \\
\hline 0.1503 & 3.0674 \\
\hline 0.1762 & 3.5745 \\
\hline 0.2004 & 4.0627 \\
\hline 0.2251 & 4.5614 \\
\hline 0.2507 & 5.0705 \\
\hline
\end{tabular}

\begin{tabular}{|c|c|}
\hline Slope Coefficient (in- $\left.\mathbf{H}_{\mathbf{2}} \mathbf{O} / \mathbf{V}_{\text {DC }}\right)$ & 0.0500 \\
\hline Offset Coefficient (in- $\left.\mathbf{H}_{2} \mathbf{O}\right)$ & -0.0026 \\
\hline Non-Linearity & $\pm 0.98 \%$ Full-Scale \\
\hline
\end{tabular}


Table A.8 Pressure Transducer Specifications; Setra Serial Number: 1548165

General:

\begin{tabular}{|c|c|}
\hline Manufacturer & Setra \\
\hline Model Number & 267 \\
\hline Serial Number & 1548165 \\
\hline Range & $0-10.0^{\prime \prime} \mathrm{H}_{2} \mathrm{O}$ \\
\hline
\end{tabular}

Calibration Data:

\begin{tabular}{|c|c|}
\hline Applied Pressure (in- $\left.\mathbf{H}_{\mathbf{2}} \mathbf{O}\right)$ & Transducer Output \\
\hline 0.0999 & 0.1510 \\
\hline 1.0921 & 1.1387 \\
\hline 2.0993 & 2.1475 \\
\hline 3.0932 & 3.1567 \\
\hline 4.0888 & 4.1412 \\
\hline 5.0963 & 5.1511 \\
\hline 6.0943 & 6.1585 \\
\hline 7.0998 & 7.1555 \\
\hline 8.0985 & 8.1699 \\
\hline 9.0997 & 9.1546 \\
\hline 10.0001 & 10.0521 \\
\hline
\end{tabular}

\begin{tabular}{|c|c|}
\hline Slope Coefficient (in- $\left.\mathbf{H}_{\mathbf{2}} \mathbf{O} / \mathbf{V}_{\text {DC }}\right)$ & 0.9990 \\
\hline Offset Coefficient (in- $\left.\mathbf{H}_{\mathbf{2}} \mathbf{O}\right)$ & -0.0510 \\
\hline Non-Linearity & $\pm 0.44 \%$ Full-Scale \\
\hline
\end{tabular}


Table A.9 Pressure Transducer Specifications; Setra Serial Number: 1753678

General:

\begin{tabular}{|c|c|}
\hline Manufacturer & Setra \\
\hline Model Number & 264 \\
\hline Serial Number & 1753678 \\
\hline Range & $0-2.5^{\prime \prime} \mathrm{H}_{2} \mathrm{O}$ \\
\hline
\end{tabular}

Calibration Data:

\begin{tabular}{|c|c|}
\hline Applied Pressure (in- $\left.\mathbf{H}_{2} \mathbf{O}\right)$ & Transducer Output \\
\hline 0.0181 & 0.0953 \\
\hline 0.2748 & 0.6039 \\
\hline 0.4962 & 1.0501 \\
\hline 0.7888 & 1.6324 \\
\hline 0.9756 & 2.0064 \\
\hline 1.2621 & 2.5792 \\
\hline 1.5481 & 3.1497 \\
\hline 1.7745 & 3.6002 \\
\hline 2.0716 & 4.1943 \\
\hline 2.2738 & 4.5984 \\
\hline 2.5233 & 5.0958 \\
\hline
\end{tabular}

\begin{tabular}{|c|c|}
\hline Slope Coefficient (in- $\left.\mathbf{H}_{\mathbf{2}} \mathbf{O} / \mathbf{V}_{\mathbf{D C}}\right)$ & 0.5008 \\
\hline Offset Coefficient (in- $\left.\mathbf{H}_{\mathbf{2}} \mathbf{O}\right)$ & -0.0291 \\
\hline Non-Linearity & $\pm 0.206 \%$ Full-Scale \\
\hline
\end{tabular}


Table A.10 Pressure Transducer Specifications; Setra Serial Number: 1753679 General:

\begin{tabular}{|c|c|}
\hline Manufacturer & Setra \\
\hline Model Number & 264 \\
\hline Serial Number & 1753679 \\
\hline Range & $0-5.0^{\prime \prime} \mathrm{H}_{2} \mathrm{O}$ \\
\hline
\end{tabular}

Calibration Data:

\begin{tabular}{|c|c|}
\hline Applied Pressure (in- $\left.\mathbf{H}_{\mathbf{2}} \mathbf{O}\right)$ & Transducer Output \\
\hline 0.0498 & 0.1051 \\
\hline 0.5972 & 0.6459 \\
\hline 1.0333 & 1.0812 \\
\hline 1.4923 & 1.5421 \\
\hline 2.0214 & 2.0739 \\
\hline 2.5428 & 2.5989 \\
\hline 3.0003 & 3.0590 \\
\hline 3.5453 & 3.6066 \\
\hline 4.0281 & 4.0893 \\
\hline 4.5293 & 4.5885 \\
\hline 5.0354 & 5.0874 \\
\hline
\end{tabular}

\begin{tabular}{|c|c|}
\hline Slope Coefficient (in- $\left.\mathbf{H}_{\mathbf{2}} \mathbf{O} / \mathbf{V}_{\text {DC }}\right)$ & 0.9983 \\
\hline Offset Coefficient (in- $\left.\mathbf{H}_{2} \mathbf{O}\right)$ & -0.0503 \\
\hline Non-Linearity & $\pm 0.206 \%$ Full-Scale \\
\hline
\end{tabular}




\section{Appendix B: TSI-to-Lab Non-Orthogonal and Tilt Velocity Transformations}

This appendix shows the original non-orthogonal and tilt transformations given by TSI, the company that developed the LDV system used in this study. Detailed schematics are given documenting TSI's original coordinate system, probe orientation, and variables. Following these schematics, additional figures show the probe orientation and variables used for the lab coordinate system unique to this study. Descriptions describe the alterations made to TSI's transformations, thus allowing the determination of the proper desired quantities.

\section{TSI Nomenclature:}

$u_{1}=$ mean velocity in the $X$-direction in the tilted coordinate system
$u_{2}=$ mean velocity in the $Y$-direction in the tilted coordinate system
$u_{3}=\quad$ mean velocity in the $Z$-direction in the tilted coordinate system
$u_{b}=\quad$ mean measured velocity by the blue laser beam pair crossing
$u_{g} \quad=\quad$ mean measured velocity by the green laser beam pair crossing
$u_{v} \quad=\quad$ mean measured velocity by the violet laser beam pair crossing
$u_{x} \quad=\quad$ mean orthogonal velocity along the $X$-axis in the TSI coordinate system
$u_{y} \quad=\quad$ mean orthogonal velocity along the $Y$-axis in the TSI coordinate system
$u_{z} \quad=\quad$ mean orthogonal velocity along the $Z$-axis in the TSI coordinate system

Greek:

$$
\begin{array}{lll}
\theta= & \text { tilt angle } \\
\theta_{g}= & \begin{array}{l}
\text { probe angle off the } Y \text {-axis applied to the two-component (blue and green) } \\
\text { LDV probe }
\end{array} \\
\theta_{v}= & \begin{array}{l}
\text { probe angle off the } Y \text {-axis applied to the single-component (violet) LDV } \\
\text { probe }
\end{array}
\end{array}
$$




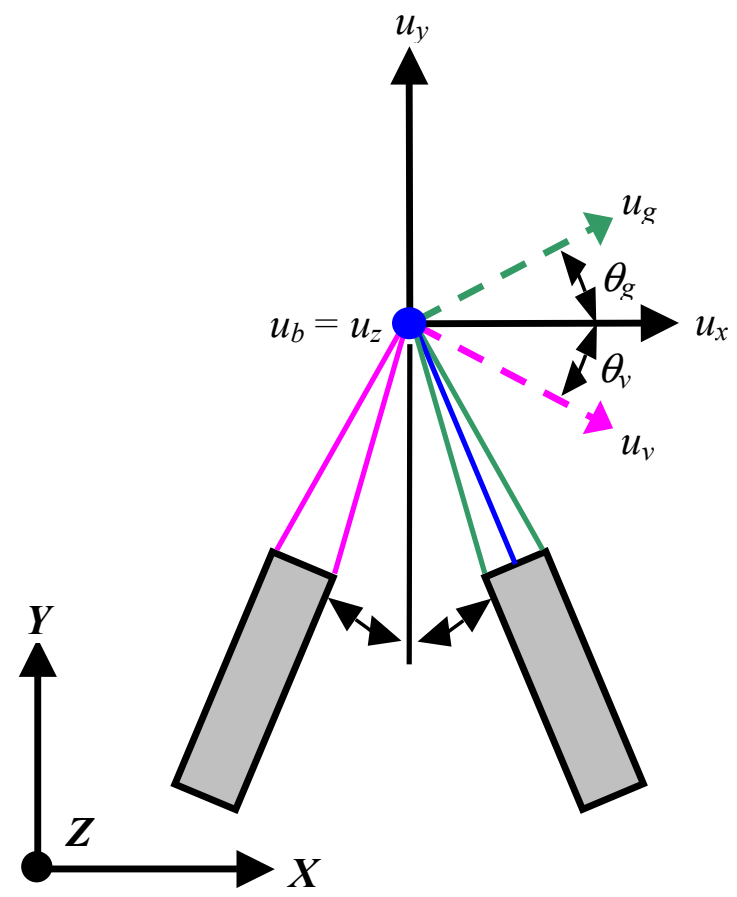

Figure B.1 TSI's recommended three-component non-orthogonal to orthogonal velocity transformation. This diagram shows their coordinate system, probe orientation, and variables (TSI, 2000).

$$
\begin{aligned}
& a_{1}=\frac{\sin \theta_{v}}{\sin \left(\theta_{g}+\theta_{v}\right)}, b_{1}=\frac{\sin \theta_{g}}{\sin \left(\theta_{g}+\theta_{v}\right)} \\
& c_{1}=\frac{\cos \theta_{v}}{\sin \left(\theta_{g}+\theta_{v}\right)}, d_{1}=\frac{-\cos \theta_{g}}{\sin \left(\theta_{g}+\theta_{v}\right)} \\
& {\left[\begin{array}{l}
u_{x} \\
u_{y} \\
u_{z}
\end{array}\right]=\left[\begin{array}{lll}
a_{1} & 0 & b_{1} \\
c_{1} & 0 & d_{1} \\
0 & 1 & 0
\end{array}\right]\left[\begin{array}{l}
u_{g} \\
u_{b} \\
u_{v}
\end{array}\right]} \\
& u_{x}=\frac{u_{g} \sin \theta_{v}+u_{v} \sin \theta_{g}}{\sin \left(\theta_{g}+\theta_{v}\right)} \\
& u_{y}=\frac{u_{g} \cos \theta_{v}-u_{v} \cos \theta_{g}}{\sin \left(\theta_{g}+\theta_{v}\right)} \\
& u_{z}=u_{b}
\end{aligned}
$$

The blue beam pair measures the component of velocity $\left(u_{z}\right)$ out of the plane of the paper, while the green and violet beam pairs measure the non-orthogonal components $\left(u_{g}\right.$ and $u_{v}$ ) of velocity. The desired quantities are the orthogonal values of $u_{x}, u_{y}$, and $u_{z}$. 


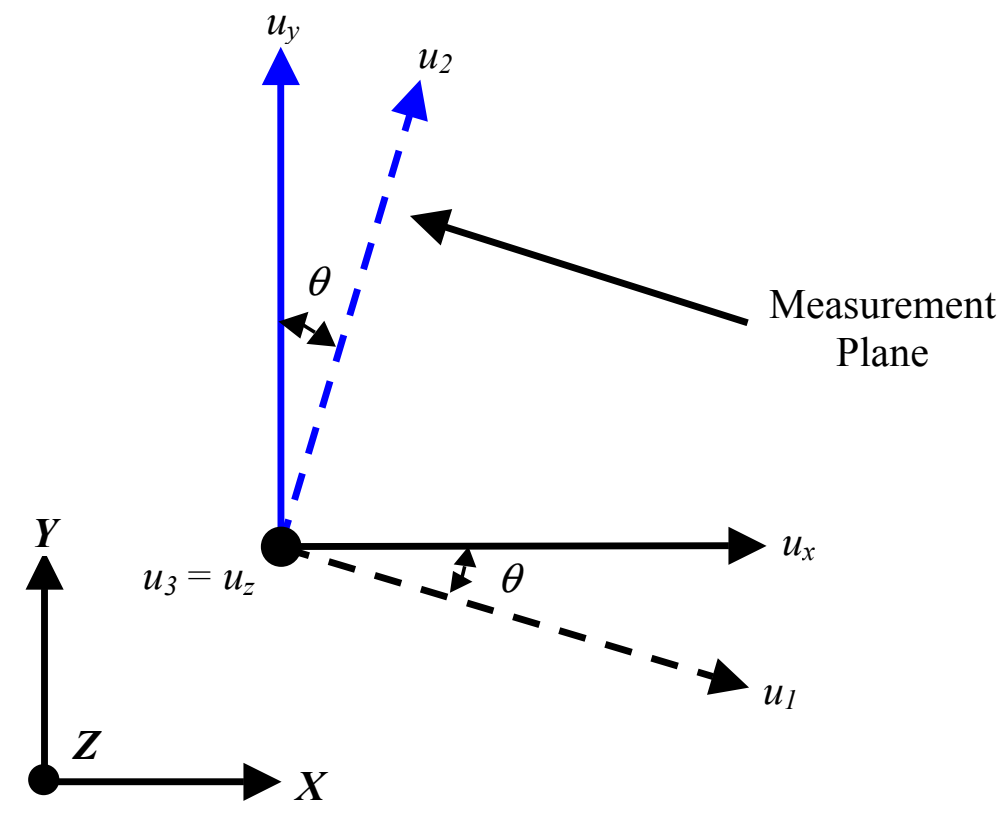

Figure B.2 TSI's recommended three-component tilt transformation, including their specific coordinate system, probe orientation, and variables (TSI, 2000).

$$
\begin{aligned}
& u_{x}=u_{1} \cos \theta+u_{2} \sin \theta \\
& u_{y}=-u_{1} \sin \theta+u_{2} \cos \theta \\
& u_{z}=u_{3}
\end{aligned}
$$

Here, $u_{1}, u_{2}$, and $u_{3}$ represent the (transformed) orthogonal components of velocity. However, the coordinate system for $u_{1}, u_{2}$, and $u_{3}$ is tilted with respect to the lab coordinate system. The desired velocity quantities are in the lab coordinate system $\left(u_{x}, u_{y}\right.$, and $\left.u_{z}\right)$. 


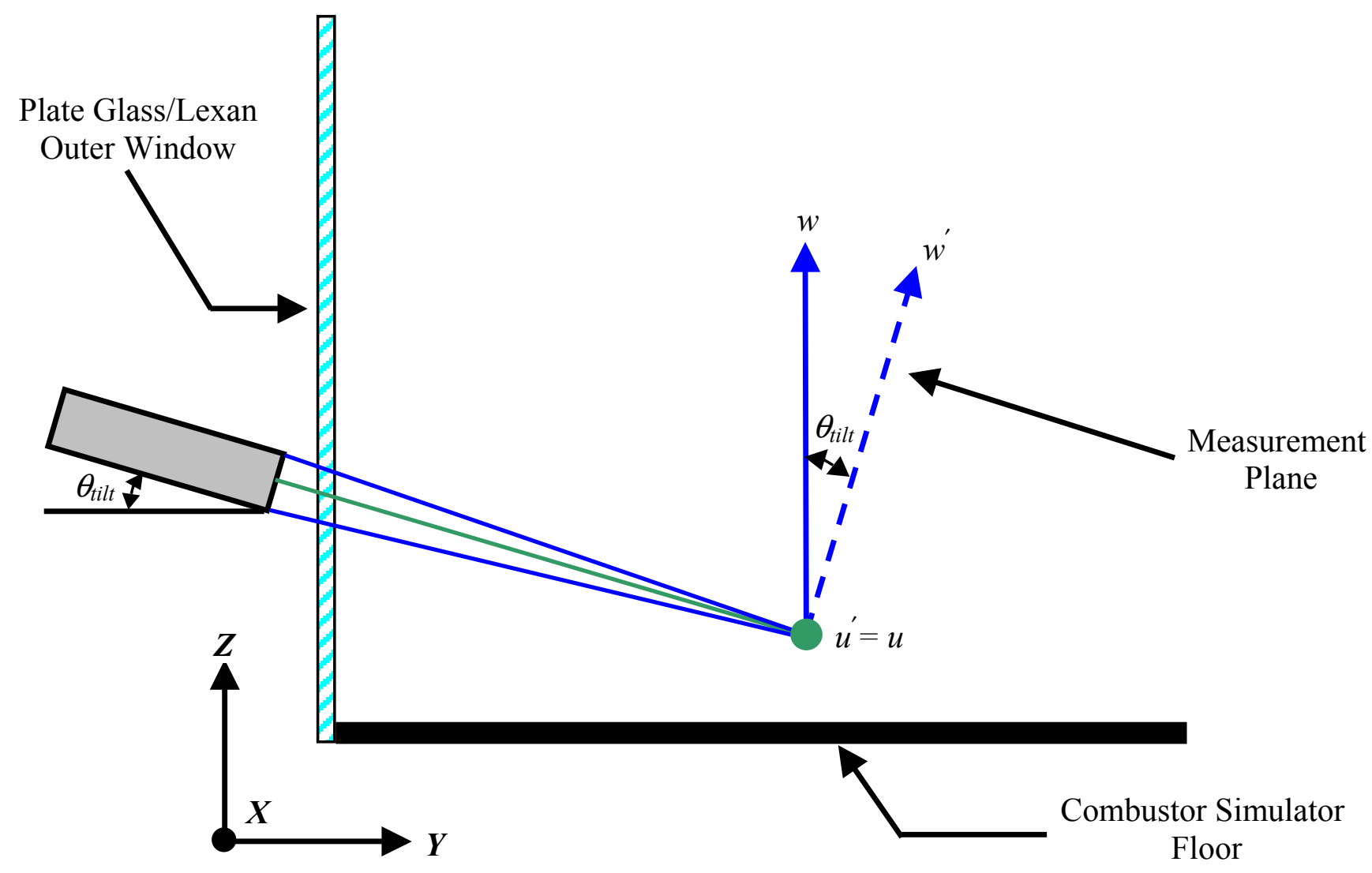

Figure B.3 The two-component transformation used in this study, including the specific coordinate system, probe orientation, and variables defined for the combustor simulator described in Chapters 3 and 4 (TSI, 2000).

$$
\begin{aligned}
& u=u^{\prime} \\
& w=\cos \left(\theta_{\text {tilt }}\right) \cdot w^{\prime}
\end{aligned}
$$

Note that the coordinate system used here is different than that of TSI's in Figure B.2. Only two-components of velocity were resolved in plane $2 \mathrm{~s}$; therefore, $u_{x}$ and $u_{1}$ in Figure B.2 and equation B. 2 are equal to zero. Lastly, $u^{\prime}, w^{\prime}$, and $\theta_{\text {tilt }}$ are equal to $u_{3}, u_{2}$, and $\theta$, respectively, in Figure B.2. 
Note: The positions of the probes are interchanged from TSI's configuration in Figure B.1. Therefore the elements of the matrix in equation B. 2 become:

$$
\left[\begin{array}{l}
u_{x} \\
u_{y} \\
u_{z}
\end{array}\right]=\left[\begin{array}{ccc}
a_{1} & 0 & b_{1} \\
-c_{1} & 0 & -d_{1} \\
0 & 1 & 0
\end{array}\right]\left[\begin{array}{l}
u_{g} \\
u_{b} \\
u_{v}
\end{array}\right]
$$
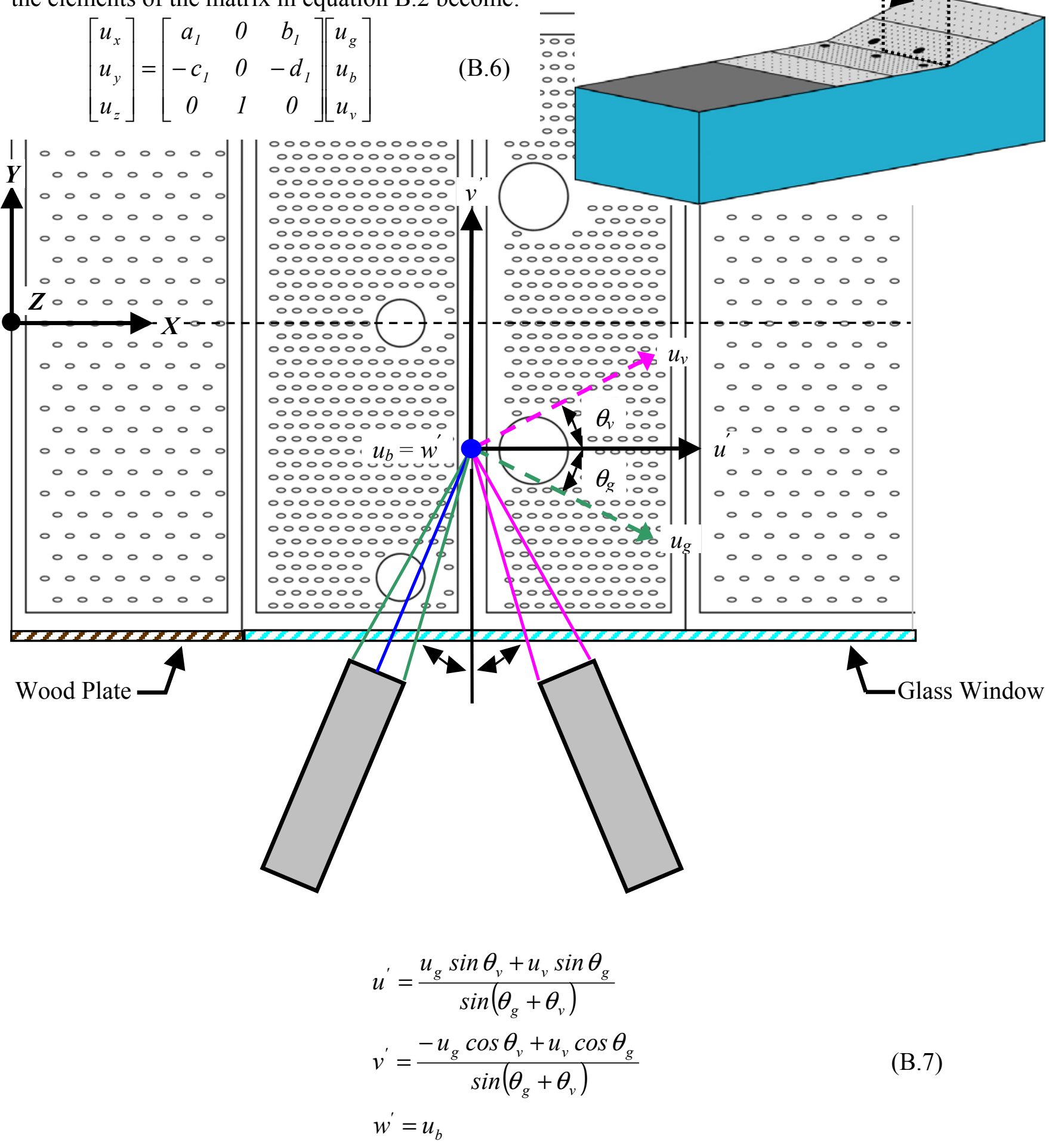

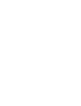
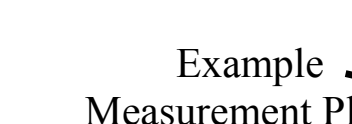

Figure B.4 Three-component non-orthogonal to orthogonal transformation used in this study. Again, showing the coordinate system, probe orientation, and variables specific to this study (TSI, 2000). 


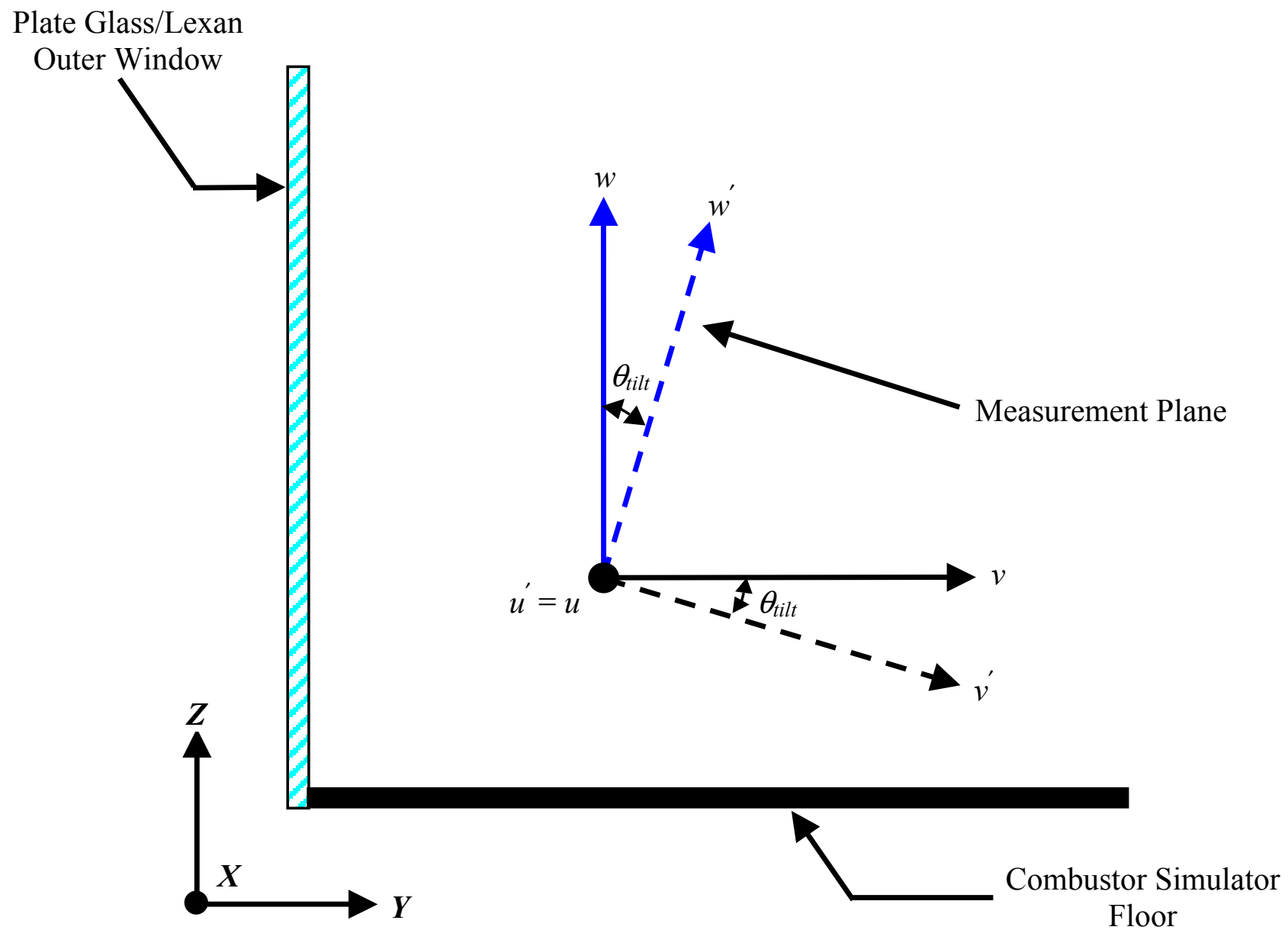

Figure B.5 The three-component tilt transformation used in this study, including the coordinate system, probe orientation, and variables specific to this study (TSI, 2000).

$$
\begin{aligned}
& u=u^{\prime} \\
& v=v^{\prime} \cos \theta_{\text {tilt }}+w^{\prime} \sin \theta_{\text {tilt }} \\
& w=-v^{\prime} \sin \theta_{\text {tilt }}+w^{\prime} \cos \theta_{\text {tilt }}
\end{aligned}
$$

Again, the coordinate system used here is different than that of TSI's in Figure B.2. All three-components of velocity were measured for planes $1 p$ and $2 p$. Therefore, $u^{\prime}, v^{\prime}, w^{\prime}$, and $\theta_{\text {tilt }}$ are equal to $u_{3}, u_{1}, u_{2}$, and $\theta$, respectively, in Figure B.2. 


\section{Appendix C: Uncertainty Calculations}

This appendix shows the uncertainty analysis calculations. Note that these calculations show the intermediate variables and their respective uncertainties. Then these intermediate variables and uncertainties are used to calculate the final variable and its uncertainty. For example, when determining the uncertainty in the normalized streamwise velocity, $u / u_{i n}$, which was taken using the three-component LDV system, the uncertainties in $u$ and $u$ are calculated before finally determining the overall uncertainty in the normalized streamwise component of velocity.

\section{Uncertainty Nomenclature:}

$U=$ The total uncertainty in a variable including precisian and bias uncertainties. The particular variable for which the uncertainty pertains to is denoted by the subscript.

$u_{\text {norm }}=$ normalized mean streamwise velocity, $u / u_{\text {in }}$

$u_{r m s-n o r m}=$ normalized root-mean-square of the streamwise velocity, $u_{r m s} / u_{\text {in }}$

$v_{\text {norm }}=$ normalized mean pitchwise velocity, $v / u_{\text {in }}$

$v_{r m s-n o r m}=$ normalized root-mean-square of the pitchwise velocity, $v_{r m s} / u_{i n}$

$w_{\text {norm }}=$ normalized mean spanwise velocity, $w / u_{\text {in }}$

$w_{\text {rms-norm }}=$ normalized root-mean-square of the spanwise velocity, $w_{r m s} / u_{\text {in }}$ 
Thermal Field Measurements, $\theta$

Equations:

$$
\begin{gathered}
\theta=\frac{T_{\infty}-T}{T_{\infty}-T_{c}} \\
\frac{\partial \theta}{\partial T}=\frac{1}{T_{c}-T_{\infty}} \\
\frac{\partial \theta}{\partial T_{\infty}}=\frac{T-T_{c}}{\left(T_{c}-T_{\infty}\right)^{2}} \\
\frac{\partial \theta}{\partial T_{c}}=\frac{T_{\infty}-T}{\left(T_{c}-T_{\infty}\right)^{2}} \\
U_{\theta}=\sqrt{\left(\frac{\partial \theta}{\partial T} \cdot U_{T}\right)^{2}+\left(\frac{\partial \theta}{\partial T_{\infty}} \cdot U_{T_{\infty}}\right)^{2}+\left(\frac{\partial \theta}{\partial T_{c}} \cdot U_{T_{c}}\right)^{2}}
\end{gathered}
$$

High Value of $\theta$

Value Location: Plane $2 \mathrm{~s}(0.538,-0.250,0.059)$

\begin{tabular}{|c|c|c|c|c|}
\hline Variable & Value & $\begin{array}{c}\text { Precision } \\
\text { Uncertainty }\end{array}$ & $\begin{array}{c}\text { Bias } \\
\text { Uncertainty }\end{array}$ & $\begin{array}{c}\text { Total } \\
\text { Uncertainty }\end{array}$ \\
\hline$\theta$ & 0.92 & - & - & 0.016 \\
\hline$T\left({ }^{\circ} \mathrm{C}\right)$ & 25.08 & 0.40 & 0.20 & 0.45 \\
\hline$T_{\infty}\left({ }^{\circ} \mathrm{C}\right)$ & 53.54 & 0.36 & 0.20 & 0.41 \\
\hline$T_{c}\left({ }^{\circ} \mathrm{C}\right)$ & 22.10 & 0.36 & 0.20 & 0.41 \\
\hline
\end{tabular}

Low Value of $\theta$

Value Location: Plane 2s (0.499, -0.250, 0.097)

\begin{tabular}{|c|c|c|c|c|}
\hline Variable & Value & $\begin{array}{c}\text { Precision } \\
\text { Uncertainty }\end{array}$ & $\begin{array}{c}\text { Bias } \\
\text { Uncertainty }\end{array}$ & $\begin{array}{c}\text { Total } \\
\text { Uncertainty }\end{array}$ \\
\hline$\theta$ & 0.044 & - & - & 0.016 \\
\hline$T\left({ }^{\circ} \mathrm{C}\right)$ & 56.93 & 0.40 & 0.20 & 0.45 \\
\hline$T_{\infty}\left({ }^{\circ} \mathrm{C}\right)$ & 58.54 & 0.36 & 0.20 & 0.41 \\
\hline$T_{c}\left({ }^{\circ} \mathrm{C}\right)$ & 22.10 & 0.36 & 0.20 & 0.41 \\
\hline
\end{tabular}


Streamwise Velocity, $u$, for Two-Component LDV Measurements (Tilt Transformation Only)

Equations:

$$
u=u^{\prime}
$$

High Value of $u$

Value Location: Plane 2s $(0.808,-0.250,0.137)$

\begin{tabular}{|c|c|c|c|c|}
\hline Variable & Value & $\begin{array}{c}\text { Precision } \\
\text { Uncertainty }\end{array}$ & $\begin{array}{c}\text { Bias } \\
\text { Uncertainty }\end{array}$ & $\begin{array}{c}\text { Total } \\
\text { Uncertainty }\end{array}$ \\
\hline$u(\mathrm{~m} / \mathrm{s})$ & 9.97 & 0.13 & 0.10 & $0.16(1.6 \%)$ \\
\hline
\end{tabular}

Low Value of $u$

Value Location: Plane 2s $(0.600,-0.250,0.065)$

\begin{tabular}{|c|c|c|c|c|}
\hline Variable & Value & $\begin{array}{c}\text { Precision } \\
\text { Uncertainty }\end{array}$ & $\begin{array}{c}\text { Bias } \\
\text { Uncertainty }\end{array}$ & $\begin{array}{c}\text { Total } \\
\text { Uncertainty }\end{array}$ \\
\hline$u(\mathrm{~m} / \mathrm{s})$ & -2.87 & 0.13 & 0.03 & $0.13(4.5 \%)$ \\
\hline
\end{tabular}


Normalized Streamwise Velocity, $u_{\text {norm }}$, for Two-Component LDV Measurements (Tilt Transformation Only)

Equations:

$$
\begin{aligned}
& u_{\text {norm }}=\frac{u}{u_{\text {in }}} \\
& \frac{\partial u_{\text {norm }}}{\partial u}=\frac{1}{u_{\text {in }}} \\
& \frac{\partial u_{\text {norm }}}{\partial u_{\text {in }}}=\frac{-u}{u_{\text {in }}^{2}} \\
& U_{u_{\text {norm }}}=\sqrt{\left(\frac{\partial u_{\text {norm }}}{\partial u} \cdot U_{u}\right)^{2}+\left(\frac{\partial u_{\text {norm }}}{\partial u_{\text {in }}} \cdot U_{u_{\text {in }}}\right)^{2}}
\end{aligned}
$$

High Value of $u_{\text {norm }}$

Value Location: Plane 2s $(0.808,-0.250,0.137)$

\begin{tabular}{|c|c|c|c|c|}
\hline Variable & Value & $\begin{array}{c}\text { Precision } \\
\text { Uncertainty }\end{array}$ & $\begin{array}{c}\text { Bias } \\
\text { Uncertainty }\end{array}$ & $\begin{array}{c}\text { Total } \\
\text { Uncertainty }\end{array}$ \\
\hline$u_{\text {norm }}$ & 5.97 & - & - & $0.46(7.8 \%)$ \\
\hline$u(\mathrm{~m} / \mathrm{s})$ & 9.97 & - & - & 0.16 \\
\hline$u_{\text {in }}(\mathrm{m} / \mathrm{s})$ & 1.67 & 0.13 & 0.02 & 0.13 \\
\hline
\end{tabular}

Low Value of $u_{\text {norm }}$

Value Location: Plane $2 \mathrm{~s}(0.600,-0.250,0.065)$

\begin{tabular}{|c|c|c|c|c|}
\hline Variable & Value & $\begin{array}{c}\text { Precision } \\
\text { Uncertainty }\end{array}$ & $\begin{array}{c}\text { Bias } \\
\text { Uncertainty }\end{array}$ & $\begin{array}{c}\text { Total } \\
\text { Uncertainty }\end{array}$ \\
\hline$u_{\text {norm }}$ & -1.72 & - & - & $0.15(8.8 \%)$ \\
\hline$u(\mathrm{~m} / \mathrm{s})$ & -2.87 & - & - & 0.13 \\
\hline$u_{\text {in }}(\mathrm{m} / \mathrm{s})$ & 1.67 & 0.13 & 0.02 & 0.13 \\
\hline
\end{tabular}


Spanwise Velocity, $w$, for Two-Component LDV Measurements (Tilt Transformation Only)

Equations:

$$
\begin{aligned}
& w=\cos \left(\theta_{\text {tilt }}\right) \cdot w^{\prime} \\
& \frac{\partial w}{\partial \theta_{\text {tilt }}}=-w^{\prime} \sin \left(\theta_{\text {tilt }}\right) \\
& \frac{\partial w}{\partial w^{\prime}}=\cos \left(\theta_{\text {tilt }}\right) \\
& U_{w}=\sqrt{\left(\frac{\partial w}{\partial \theta_{\text {tilt }}} \cdot U_{\theta_{\text {tilt }}}\right)^{2}+\left(\frac{\partial w}{\partial w^{\prime}} \cdot U_{w^{\prime}}\right)^{2}}
\end{aligned}
$$

High Value of $w$

Value Location: Plane 2s (0.600, -0.250, 0.057)

\begin{tabular}{|c|c|c|c|c|}
\hline Variable & Value & $\begin{array}{c}\text { Precision } \\
\text { Uncertainty }\end{array}$ & $\begin{array}{c}\text { Bias } \\
\text { Uncertainty }\end{array}$ & $\begin{array}{c}\text { Total } \\
\text { Uncertainty }\end{array}$ \\
\hline$w(\mathrm{~m} / \mathrm{s})$ & 12.67 & - & - & $0.15(1.2 \%)$ \\
\hline$\theta_{\text {tilt }}$ & $7.33^{\circ}$ & - & $1.00^{\circ}$ & $1.00^{\circ}$ \\
\hline$w^{\prime}(\mathrm{m} / \mathrm{s})$ & 12.77 & 0.074 & 0.13 & 0.15 \\
\hline
\end{tabular}

Low Value of $w$

Value Location: Plane 2s $(0.509,-0.250,0.207)$

\begin{tabular}{|c|c|c|c|c|}
\hline Variable & Value & $\begin{array}{c}\text { Precision } \\
\text { Uncertainty }\end{array}$ & $\begin{array}{c}\text { Bias } \\
\text { Uncertainty }\end{array}$ & $\begin{array}{c}\text { Total } \\
\text { Uncertainty }\end{array}$ \\
\hline$w^{(\mathrm{m} / \mathrm{s})}$ & -1.30 & - & - & $0.075(5.7 \%)$ \\
\hline$\theta_{\text {tilt }}$ & $7.33^{\circ}$ & - & $1.00^{\circ}$ & $1.00^{\circ}$ \\
\hline$w^{\prime}(\mathrm{m} / \mathrm{s})$ & -1.31 & 0.074 & 0.013 & 0.075 \\
\hline
\end{tabular}


Normalized Spanwise Velocity, $w_{\text {norm }}$, for Two-Component LDV Measurements (Tilt Transformation Only)

Equations:

$$
\begin{aligned}
& w_{\text {norm }}=\frac{w}{u_{\text {in }}} \\
& \frac{\partial w_{\text {norm }}}{\partial w}=\frac{1}{u_{\text {in }}} \\
& \frac{\partial w_{\text {norm }}}{\partial u_{\text {in }}}=\frac{-w}{u_{\text {in }}^{2}} \\
& U_{w_{\text {norm }}}=\sqrt{\left(\frac{\partial w_{\text {norm }}}{\partial w} \cdot U_{w}\right)^{2}+\left(\frac{\partial w_{\text {norm }}}{\partial u_{\text {in }}} \cdot U_{u_{\text {in }}}\right)^{2}}
\end{aligned}
$$

High Value of $w_{\text {norm }}$

Value Location: Plane 2s $(0.600,-0.250,0.057)$

\begin{tabular}{|c|c|c|c|c|}
\hline Variable & Value & $\begin{array}{c}\text { Precision } \\
\text { Uncertainty }\end{array}$ & $\begin{array}{c}\text { Bias } \\
\text { Uncertainty }\end{array}$ & $\begin{array}{c}\text { Total } \\
\text { Uncertainty }\end{array}$ \\
\hline$w_{\text {norm }}$ & 7.59 & - & - & $0.58(7.7 \%)$ \\
\hline$w(\mathrm{~m} / \mathrm{s})$ & 12.67 & - & - & 0.15 \\
\hline$u_{\text {in }}(\mathrm{m} / \mathrm{s})$ & 1.67 & 0.13 & 0.017 & 0.13 \\
\hline
\end{tabular}

Low Value of $w_{\text {norm }}$

Value Location: Plane 2s $(0.509,-0.250,0.207)$

\begin{tabular}{|c|c|c|c|c|}
\hline Variable & Value & $\begin{array}{c}\text { Precision } \\
\text { Uncertainty }\end{array}$ & $\begin{array}{c}\text { Bias } \\
\text { Uncertainty }\end{array}$ & $\begin{array}{c}\text { Total } \\
\text { Uncertainty }\end{array}$ \\
\hline$w_{\text {norm }}$ & -0.78 & - & - & $0.074(9.5 \%)$ \\
\hline$w(\mathrm{~m} / \mathrm{s})$ & -1.3 & - & - & 0.075 \\
\hline$u_{\text {in }}(\mathrm{m} / \mathrm{s})$ & 1.67 & 0.13 & 0.017 & 0.13 \\
\hline
\end{tabular}


Streamwise Velocity, $u^{\prime}$, for Three-Component LDV Measurements

(Non-Orthogonal Transformation Only)

Equations:

$$
\begin{aligned}
& u^{\prime}=\frac{u_{g} \sin \theta_{v}+u_{v} \sin \theta_{g}}{\sin \left(\theta_{g}+\theta_{v}\right)} \\
& \frac{\partial u^{\prime}}{\partial u_{g}}=\frac{\sin \theta_{v}}{\sin \left(\theta_{g}+\theta_{v}\right)} \\
& \frac{\partial u^{\prime}}{\partial u_{v}}=\frac{\sin \theta_{g}}{\sin \left(\theta_{g}+\theta_{v}\right)} \\
& \frac{\partial u^{\prime}}{\partial \theta_{g}}=\frac{\left(u_{v}-u_{g} \cos \left(\theta_{g}+\theta_{v}\right)\right) \sin \theta_{v}}{\sin ^{2}\left(\theta_{g}+\theta_{v}\right)} \\
& \frac{\partial u^{\prime}}{\partial \theta_{v}}=\frac{\left(u_{g}-u_{v} \cos \left(\theta_{g}+\theta_{v}\right)\right) \sin \theta_{g}}{\sin ^{2}\left(\theta_{g}+\theta_{v}\right)} \\
& U_{u^{\prime}}=\sqrt{\left(\frac{\partial u^{\prime}}{\partial u_{g}} \cdot U_{u_{g}}\right)^{2}+\left(\frac{\partial u^{\prime}}{\partial u_{v}} \cdot U_{u_{v}}\right)^{2}+\left(\frac{\partial u^{\prime}}{\partial \theta_{g}} \cdot U_{\theta_{g}}\right)^{2}+\left(\frac{\partial u^{\prime}}{\partial \theta_{v}} \cdot U_{\theta_{v}}\right)^{2}}
\end{aligned}
$$

High Value of $u$

Value Location: Plane $1 p(0.500,-0.438,0.131)$

\begin{tabular}{|c|c|c|c|c|}
\hline Variable & Value & $\begin{array}{c}\text { Precision } \\
\text { Uncertainty }\end{array}$ & $\begin{array}{c}\text { Bias } \\
\text { Uncertainty }\end{array}$ & $\begin{array}{c}\text { Total } \\
\text { Uncertainty }\end{array}$ \\
\hline$u^{\prime}(\mathrm{m} / \mathrm{s})$ & 6.88 & - & - & $0.11(1.5 \%)$ \\
\hline$u_{g}(\mathrm{~m} / \mathrm{s})$ & 6.86 & 0.13 & 0.069 & 0.14 \\
\hline$u_{v}(\mathrm{~m} / \mathrm{s})$ & 6.52 & 0.13 & 0.065 & 0.14 \\
\hline$\theta_{g}$ & $13.33^{\circ}$ & - & $1.00^{\circ}$ & $1.00^{\circ}$ \\
\hline$\theta_{v}$ & $13.33^{\circ}$ & - & $1.00^{\circ}$ & $1.00^{\circ}$ \\
\hline
\end{tabular}


Low Value of $u^{\prime}$

Value Location: Plane $1 p(0.500,0.0,0.156)$

\begin{tabular}{|c|c|c|c|c|}
\hline Variable & Value & $\begin{array}{c}\text { Precision } \\
\text { Uncertainty }\end{array}$ & $\begin{array}{c}\text { Bias } \\
\text { Uncertainty }\end{array}$ & $\begin{array}{c}\text { Total } \\
\text { Uncertainty }\end{array}$ \\
\hline$u^{\prime}(\mathrm{m} / \mathrm{s})$ & -0.96 & - & - & $0.092(9.6 \%)$ \\
\hline$u_{g}(\mathrm{~m} / \mathrm{s})$ & -1.10 & 0.13 & 0.011 & 0.13 \\
\hline$u_{v}(\mathrm{~m} / \mathrm{s})$ & -0.76 & 0.13 & 0.0080 & 0.13 \\
\hline$\theta_{g}$ & $13.33^{\circ}$ & - & $1.00^{\circ}$ & $1.00^{\circ}$ \\
\hline$\theta_{v}$ & $13.33^{\circ}$ & - & $1.00^{\circ}$ & $1.00^{\circ}$ \\
\hline
\end{tabular}


Streamwise Velocity, $u$, for Three-Component LDV Measurements (Non-Orthogonal and Tilt Transformations)

Equations:

$$
u=u^{\prime}
$$

High Value of $u$

Value Location: Plane $1 p(0.500,-0.438,0.131)$

\begin{tabular}{|c|c|c|c|c|}
\hline Variable & Value & $\begin{array}{c}\text { Precision } \\
\text { Uncertainty }\end{array}$ & $\begin{array}{c}\text { Bias } \\
\text { Uncertainty }\end{array}$ & $\begin{array}{c}\text { Total } \\
\text { Uncertainty }\end{array}$ \\
\hline$u(\mathrm{~m} / \mathrm{s})$ & 6.88 & - & - & $0.11(1.5 \%)$ \\
\hline
\end{tabular}

Low Value of $u$

Value Location: Plane 1p (0.500, 0.0, 0.156)

\begin{tabular}{|c|c|c|c|c|}
\hline Variable & Value & $\begin{array}{c}\text { Precision } \\
\text { Uncertainty }\end{array}$ & $\begin{array}{c}\text { Bias } \\
\text { Uncertainty }\end{array}$ & $\begin{array}{c}\text { Total } \\
\text { Uncertainty }\end{array}$ \\
\hline$u(\mathrm{~m} / \mathrm{s})$ & -0.96 & - & - & $0.092(9.6 \%)$ \\
\hline
\end{tabular}


Normalized Streamwise Velocity, $\boldsymbol{u}_{\text {norm}}$, for Three-Component LDV Measurements (Non-Orthogonal and Tilt Transformations)

Equations:

$$
\begin{aligned}
& u_{\text {norm }}=\frac{u}{u_{\text {in }}} \\
& \frac{\partial u_{\text {norm }}}{\partial u}=\frac{1}{u_{\text {in }}} \\
& \frac{\partial u_{\text {norm }}}{\partial u_{\text {in }}}=\frac{-u}{u_{\text {in }}^{2}} \\
& U_{u_{\text {norm }}}=\sqrt{\left(\frac{\partial u_{\text {norm }}}{\partial u} \cdot U_{u}\right)^{2}+\left(\frac{\partial u_{\text {norm }}}{\partial u_{\text {in }}} \cdot U_{u_{\text {in }}}\right)^{2}}
\end{aligned}
$$

High Value of $u_{\text {norm }}$

Value Location: Plane $1 p(0.500,-0.438,0.131)$

\begin{tabular}{|c|c|c|c|c|}
\hline Variable & Value & $\begin{array}{c}\text { Precision } \\
\text { Uncertainty }\end{array}$ & $\begin{array}{c}\text { Bias } \\
\text { Uncertainty }\end{array}$ & $\begin{array}{c}\text { Total } \\
\text { Uncertainty }\end{array}$ \\
\hline$u_{\text {norm }}$ & 4.12 & - & - & $0.32(7.7 \%)$ \\
\hline$u(\mathrm{~m} / \mathrm{s})$ & 6.88 & - & - & 0.11 \\
\hline$u_{\text {in }}(\mathrm{m} / \mathrm{s})$ & 1.67 & 0.13 & 0.017 & 0.13 \\
\hline
\end{tabular}

Low Value of $u_{\text {norm }}$

Value Location: Plane $1 \mathrm{p}(0.500,0.0,0.156)$

\begin{tabular}{|c|c|c|c|c|}
\hline Variable & Value & $\begin{array}{c}\text { Precision } \\
\text { Uncertainty }\end{array}$ & $\begin{array}{c}\text { Bias } \\
\text { Uncertainty }\end{array}$ & $\begin{array}{c}\text { Total } \\
\text { Uncertainty }\end{array}$ \\
\hline$u_{\text {norm }}$ & -0.57 & - & - & $0.070(12.3 \%)$ \\
\hline$u(\mathrm{~m} / \mathrm{s})$ & -0.96 & - & - & 0.092 \\
\hline$u_{\text {in }}(\mathrm{m} / \mathrm{s})$ & 1.67 & 0.13 & 0.017 & 0.13 \\
\hline
\end{tabular}


Pitchwise Velocity, $v^{\prime}$, for Three-Component LDV Measurements

(Non-Orthogonal Transformation Only)

Equations:

$$
\begin{gathered}
v^{\prime}=\frac{-u_{g} \cos \theta_{v}+u_{v} \cos \theta_{g}}{\sin \left(\theta_{g}+\theta_{v}\right)} \\
\frac{\partial v^{\prime}}{\partial u_{g}}=\frac{-\cos \theta_{v}}{\sin \left(\theta_{g}+\theta_{v}\right)} \\
\frac{\partial v^{\prime}}{\partial u_{v}}=\frac{\cos \theta_{g}}{\sin \left(\theta_{g}+\theta_{v}\right)} \\
\frac{\partial v^{\prime}}{\partial \theta_{g}}=\frac{\left(-u_{v}+u_{g} \cos \left(\theta_{g}+\theta_{v}\right)\right) \cos \theta_{v}}{\sin ^{2}\left(\theta_{g}+\theta_{v}\right)} \\
\frac{\partial v^{\prime}}{\partial \theta_{v}}=\frac{\left(u_{g}-u_{v} \cos \left(\theta_{g}+\theta_{v}\right)\right) \cos \theta_{g}}{\sin ^{2}\left(\theta_{g}+\theta_{v}\right)} \\
U_{v^{\prime}}=\sqrt{\left(\frac{\partial v^{\prime}}{\partial u_{g}} \cdot U_{u_{g}}\right)^{2}+\left(\frac{\partial v^{\prime}}{\partial u_{v}} \cdot U_{u_{v}}\right)^{2}+\left(\frac{\partial v^{\prime}}{\partial \theta_{g}} \cdot U_{\theta_{g}}\right)^{2}+\left(\frac{\partial v^{\prime}}{\partial \theta_{v}} \cdot U_{\theta_{v}}\right)^{2}}
\end{gathered}
$$

High Value of $v^{\prime}$

Value Location: Plane $1 p(0.500,-0.076,0.131)$

\begin{tabular}{|c|c|c|c|c|}
\hline Variable & Value & $\begin{array}{c}\text { Precision } \\
\text { Uncertainty }\end{array}$ & $\begin{array}{c}\text { Bias } \\
\text { Uncertainty }\end{array}$ & $\begin{array}{c}\text { Total } \\
\text { Uncertainty }\end{array}$ \\
\hline$v^{\prime}(\mathrm{m} / \mathrm{s})$ & 2.95 & - & - & $0.43(14.5 \%)$ \\
\hline$u_{g}(\mathrm{~m} / \mathrm{s})$ & 2.46 & 0.13 & 0.025 & 0.13 \\
\hline$u_{v}(\mathrm{~m} / \mathrm{s})$ & 3.82 & 0.13 & 0.038 & 0.13 \\
\hline$\theta_{g}$ & $13.33^{\circ}$ & - & $1.00^{\circ}$ & $1.00^{\circ}$ \\
\hline$\theta_{v}$ & $13.33^{\circ}$ & - & $1.00^{\circ}$ & $1.00^{\circ}$ \\
\hline
\end{tabular}


Low Value of $v^{\prime}$

Value Location: Plane $1 p(0.500,-0.500,0.065)$

\begin{tabular}{|c|c|c|c|c|}
\hline Variable & Value & $\begin{array}{c}\text { Precision } \\
\text { Uncertainty }\end{array}$ & $\begin{array}{c}\text { Bias } \\
\text { Uncertainty }\end{array}$ & $\begin{array}{c}\text { Total } \\
\text { Uncertainty }\end{array}$ \\
\hline$v^{\prime}(\mathrm{m} / \mathrm{s})$ & -4.51 & - & - & $0.46(10.2 \%)$ \\
\hline$u_{g}(\mathrm{~m} / \mathrm{s})$ & 3.32 & 0.13 & 0.033 & 0.13 \\
\hline$u_{v}(\mathrm{~m} / \mathrm{s})$ & 1.24 & 0.13 & 0.012 & 0.13 \\
\hline$\theta_{g}$ & $13.33^{\circ}$ & - & $1.00^{\circ}$ & $1.00^{\circ}$ \\
\hline$\theta_{v}$ & $13.33^{\circ}$ & - & $1.00^{\circ}$ & $1.00^{\circ}$ \\
\hline
\end{tabular}


Pitchwise Velocity, $v$, for Three-Component LDV Measurements (Non-Orthogonal and Tilt Transformations)

Equations:

$$
\begin{gathered}
v=v^{\prime} \cos \theta_{\text {tilt }}+w^{\prime} \sin \theta_{\text {tilt }} \\
\frac{\partial v}{\partial v^{\prime}}=\cos \theta_{\text {tilt }} \\
\frac{\partial v}{\partial w^{\prime}}=\sin \theta_{\text {tilt }} \\
\frac{\partial v}{\partial \theta_{\text {tilt }}}=w^{\prime} \cos \theta_{\text {tilt }}-v^{\prime} \sin \theta_{\text {tilt }} \\
U_{v}=\sqrt{\left(\frac{\partial v}{\partial v^{\prime}} \cdot U_{v^{\prime}}\right)^{2}+\left(\frac{\partial v}{\partial w^{\prime}} \cdot U_{w^{\prime}}\right)^{2}+\left(\frac{\partial v}{\partial \theta_{\text {tilt }}} \cdot U_{\theta_{\text {tile }}}\right)^{2}}
\end{gathered}
$$

High Value of $v$

Value Location: Plane $1 p(0.500,-0.076,0.131)$

\begin{tabular}{|c|c|c|c|c|}
\hline Variable & Value & $\begin{array}{c}\text { Precision } \\
\text { Uncertainty }\end{array}$ & $\begin{array}{c}\text { Bias } \\
\text { Uncertainty }\end{array}$ & $\begin{array}{c}\text { Total } \\
\text { Uncertainty }\end{array}$ \\
\hline$v(\mathrm{~m} / \mathrm{s})$ & 3.26 & - & - & $0.43(13.1 \%)$ \\
\hline$v^{\prime}(\mathrm{m} / \mathrm{s})$ & 2.95 & - & - & 0.43 \\
\hline$w^{\prime}(\mathrm{m} / \mathrm{s})$ & 2.64 & 0.074 & 0.026 & 0.079 \\
\hline$\theta_{\text {tilt }}$ & $7.33^{\circ}$ & - & $1.00^{\circ}$ & $1.00^{\circ}$ \\
\hline
\end{tabular}

Low Value of $v$

Value Location: Plane $1 p(0.500,-0.500,0.065)$

\begin{tabular}{|c|c|c|c|c|}
\hline Variable & Value & $\begin{array}{c}\text { Precision } \\
\text { Uncertainty }\end{array}$ & $\begin{array}{c}\text { Bias } \\
\text { Uncertainty }\end{array}$ & $\begin{array}{c}\text { Total } \\
\text { Uncertainty }\end{array}$ \\
\hline$v(\mathrm{~m} / \mathrm{s})$ & -4.15 & - & - & $0.46(11.0 \%)$ \\
\hline$v^{\prime}(\mathrm{m} / \mathrm{s})$ & -4.51 & - & - & 0.46 \\
\hline$w^{\prime}(\mathrm{m} / \mathrm{s})$ & 2.56 & 0.074 & 0.026 & 0.079 \\
\hline$\theta_{t i l t}$ & $7.33^{\circ}$ & - & $1.00^{\circ}$ & $1.00^{\circ}$ \\
\hline
\end{tabular}


Normalized Pitchwise Velocity, $v_{\text {norm }}$, for Three-Component LDV Measurements (Non-Orthogonal and Tilt Transformations)

Equations:

$$
\begin{aligned}
& v_{\text {norm }}=\frac{v}{u_{\text {in }}} \\
& \frac{\partial v_{\text {norm }}}{\partial v}=\frac{1}{u_{\text {in }}} \\
& \frac{\partial v_{\text {norm }}}{\partial u_{\text {in }}}=\frac{-v}{u_{\text {in }}^{2}} \\
& U_{v_{\text {norm }}}=\sqrt{\left(\frac{\partial v_{\text {norm }}}{\partial v} \cdot U_{v}\right)^{2}+\left(\frac{\partial v_{\text {norm }}}{\partial u_{\text {in }}} \cdot U_{u_{\text {in }}}\right)^{2}}
\end{aligned}
$$

High Value of $v_{\text {norm }}$

Value Location: Plane $1 p(0.500,-0.076,0.131)$

\begin{tabular}{|c|c|c|c|c|}
\hline Variable & Value & $\begin{array}{c}\text { Precision } \\
\text { Uncertainty }\end{array}$ & $\begin{array}{c}\text { Bias } \\
\text { Uncertainty }\end{array}$ & $\begin{array}{c}\text { Total } \\
\text { Uncertainty }\end{array}$ \\
\hline$v_{\text {norm }}$ & 1.95 & - & - & $0.30(15.1 \%)$ \\
\hline$v(\mathrm{~m} / \mathrm{s})$ & 3.26 & - & - & 0.43 \\
\hline$u_{\text {in }}(\mathrm{m} / \mathrm{s})$ & 1.67 & 0.13 & 0.017 & 0.13 \\
\hline
\end{tabular}

Low Value of $v_{\text {norm }}$

Value Location: Plane $1 p(0.500,-0.500,0.065)$

\begin{tabular}{|c|c|c|c|c|}
\hline Variable & Value & $\begin{array}{c}\text { Precision } \\
\text { Uncertainty }\end{array}$ & $\begin{array}{c}\text { Bias } \\
\text { Uncertainty }\end{array}$ & $\begin{array}{c}\text { Total } \\
\text { Uncertainty }\end{array}$ \\
\hline$v_{\text {norm }}$ & -2.48 & - & - & $0.33(13.4 \%)$ \\
\hline$v(\mathrm{~m} / \mathrm{s})$ & -4.15 & - & - & 0.46 \\
\hline$u_{\text {in }}(\mathrm{m} / \mathrm{s})$ & 1.67 & 0.13 & 0.017 & 0.127 \\
\hline
\end{tabular}


Spanwise Velocity, $w^{\prime}$, for Three-Component LDV Measurements

(Non-Orthogonal Transformation Only)

Equations:

$$
w^{\prime}=u_{b}
$$

High Value of $w^{\prime}$

Value Location: Plane $1 p(0.500,-0.438,0.232)$

\begin{tabular}{|c|c|c|c|c|}
\hline Variable & Value & $\begin{array}{c}\text { Precision } \\
\text { Uncertainty }\end{array}$ & $\begin{array}{c}\text { Bias } \\
\text { Uncertainty }\end{array}$ & $\begin{array}{c}\text { Total } \\
\text { Uncertainty }\end{array}$ \\
\hline$w^{\prime}(\mathrm{m} / \mathrm{s})$ & 5.79 & 0.074 & 0.058 & $0.094(1.6 \%)$ \\
\hline
\end{tabular}

Low Value of $w^{\prime}$

Value Location: Plane $1 p(0.500,-0.375,0.131)$

\begin{tabular}{|c|c|c|c|c|}
\hline Variable & Value & $\begin{array}{c}\text { Precision } \\
\text { Uncertainty }\end{array}$ & $\begin{array}{c}\text { Bias } \\
\text { Uncertainty }\end{array}$ & $\begin{array}{c}\text { Total } \\
\text { Uncertainty }\end{array}$ \\
\hline$w^{\prime}(\mathrm{m} / \mathrm{s})$ & -2.00 & 0.074 & 0.020 & $0.077(3.8 \%)$ \\
\hline
\end{tabular}


Spanwise Velocity, $w$, for Three-Component LDV Measurements (Non-Orthogonal and Tilt Transformations)

Equations:

$$
\begin{gathered}
w=-v^{\prime} \sin \theta_{\text {tilt }}+w^{\prime} \cos \theta_{\text {tilt }} \\
\frac{\partial w}{\partial v^{\prime}}=-\sin \theta_{\text {tilt }} \\
\frac{\partial w}{\partial w^{\prime}}=\cos \theta_{\text {tilt }} \\
\frac{\partial w}{\partial \theta_{\text {tilt }}}=-v^{\prime} \cos \theta_{\text {tilt }}-w^{\prime} \sin \theta_{\text {tilt }} \\
U_{w}=\sqrt{\left(\frac{\partial w}{\partial v^{\prime}} \cdot U_{v^{\prime}}\right)^{2}+\left(\frac{\partial w}{\partial w^{\prime}} \cdot U_{w^{\prime}}\right)^{2}+\left(\frac{\partial w}{\partial \theta_{\text {tilt }}} \cdot U_{\theta_{\text {tile }}}\right)^{2}}
\end{gathered}
$$

High Value of $w$

Value Location: Plane $1 \mathrm{p}(0.500,-0.438,0.232)$

\begin{tabular}{|c|c|c|c|c|}
\hline Variable & Value & $\begin{array}{c}\text { Precision } \\
\text { Uncertainty }\end{array}$ & $\begin{array}{c}\text { Bias } \\
\text { Uncertainty }\end{array}$ & $\begin{array}{c}\text { Total } \\
\text { Uncertainty }\end{array}$ \\
\hline$w(\mathrm{~m} / \mathrm{s})$ & 5.80 & - & - & $0.11(1.9 \%)$ \\
\hline$v^{\prime}(\mathrm{m} / \mathrm{s})$ & -0.49 & - & - & 0.42 \\
\hline$w^{\prime}(\mathrm{m} / \mathrm{s})$ & 5.79 & 0.074 & 0.058 & 0.094 \\
\hline$\theta_{\text {tilt }}$ & $7.33^{\circ}$ & - & $1.00^{\circ}$ & $1.00^{\circ}$ \\
\hline
\end{tabular}

Low Value of $w$

Value Location: Plane $1 \mathrm{p}(0.500,-0.375,0.131)$

\begin{tabular}{|c|c|c|c|c|}
\hline Variable & Value & $\begin{array}{c}\text { Precision } \\
\text { Uncertainty }\end{array}$ & $\begin{array}{c}\text { Bias } \\
\text { Uncertainty }\end{array}$ & $\begin{array}{c}\text { Total } \\
\text { Uncertainty }\end{array}$ \\
\hline$w^{\prime}(\mathrm{m} / \mathrm{s})$ & -1.81 & - & - & $0.096(5.3 \%)$ \\
\hline$v^{\prime}(\mathrm{m} / \mathrm{s})$ & -1.36 & - & - & 0.40 \\
\hline$w^{\prime}(\mathrm{m} / \mathrm{s})$ & -2.00 & 0.074 & 0.020 & 0.077 \\
\hline$\theta_{\text {tilt }}$ & $7.33^{\circ}$ & - & $1.00^{\circ}$ & $1.00^{\circ}$ \\
\hline
\end{tabular}


Normalized Spanwise Velocity, $w_{\text {norm }}$, for Three-Component LDV Measurements (Non-Orthogonal and Tilt Transformations)

Equations:

$$
\begin{aligned}
& w_{\text {norm }}=\frac{w}{u_{\text {in }}} \\
& \frac{\partial w_{\text {norm }}}{\partial w}=\frac{1}{u_{\text {in }}} \\
& \frac{\partial w_{\text {norm }}}{\partial u_{\text {in }}}=\frac{-w}{u_{\text {in }}^{2}} \\
& U_{w_{\text {norm }}}=\sqrt{\left(\frac{\partial w_{\text {norm }}}{\partial w} \cdot U_{w}\right)^{2}+\left(\frac{\partial w_{\text {norm }}}{\partial u_{\text {in }}} \cdot U_{u_{\text {in }}}\right)^{2}}
\end{aligned}
$$

High Value of $w_{\text {norm }}$

Value Location: Plane $1 p(0.500,-0.438,0.232)$

\begin{tabular}{|c|c|c|c|c|}
\hline Variable & Value & $\begin{array}{c}\text { Precision } \\
\text { Uncertainty }\end{array}$ & $\begin{array}{c}\text { Bias } \\
\text { Uncertainty }\end{array}$ & $\begin{array}{c}\text { Total } \\
\text { Uncertainty }\end{array}$ \\
\hline$w_{\text {norm }}$ & 3.47 & - & - & $0.11(7.8 \%)$ \\
\hline$w(\mathrm{~m} / \mathrm{s})$ & 5.80 & - & - & 0.11 \\
\hline$u_{\text {in }}(\mathrm{m} / \mathrm{s})$ & 1.67 & 0.13 & 0.017 & 0.13 \\
\hline
\end{tabular}

Low Value of $w_{\text {norm }}$

Value Location: Plane $1 p(0.500,-0.375,0.131)$

\begin{tabular}{|c|c|c|c|c|}
\hline Variable & Value & $\begin{array}{c}\text { Precision } \\
\text { Uncertainty }\end{array}$ & $\begin{array}{c}\text { Bias } \\
\text { Uncertainty }\end{array}$ & $\begin{array}{c}\text { Total } \\
\text { Uncertainty }\end{array}$ \\
\hline$w_{\text {norm }}$ & -1.09 & - & - & $0.10(9.3 \%)$ \\
\hline$w(\mathrm{~m} / \mathrm{s})$ & -1.81 & - & - & 0.096 \\
\hline$u_{\text {in }}(\mathrm{m} / \mathrm{s})$ & 1.67 & 0.13 & 0.017 & 0.13 \\
\hline
\end{tabular}


Normalized Streamwise RMS Levels, $\boldsymbol{u}_{\text {rms-norm }}$, for Three-Component LDV Measurements (Non-Orthogonal and Tilt Transformations)

Equations:

$$
\begin{gathered}
u_{r m s-n o r m}=\frac{u_{r m s}}{u_{i n}} \\
\frac{\partial u_{r m s-n o r m}}{\partial u_{r m s}}=\frac{1}{u_{i n}} \\
\frac{\partial u_{r m s-n o r m}}{\partial u_{i n}}=\frac{-u_{r m s}}{u_{i n}^{2}} \\
U_{u_{r m s-n o r m}}=\sqrt{\left(\frac{\partial u_{r m s-n o r m}}{\partial u_{r m s}} \cdot U_{u_{r m s}}\right)^{2}+\left(\frac{\partial u_{r m s-n o r m}}{\partial u_{i n}} \cdot U_{u_{i n}}\right)^{2}}
\end{gathered}
$$

High Value of $u_{\text {rms-norm }}$

Value Location: Plane $1 \mathrm{p}(0.500,-0.500,0.257)$

\begin{tabular}{|c|c|c|c|c|}
\hline Variable & Value & $\begin{array}{c}\text { Precision } \\
\text { Uncertainty }\end{array}$ & $\begin{array}{c}\text { Bias } \\
\text { Uncertainty }\end{array}$ & $\begin{array}{c}\text { Total } \\
\text { Uncertainty }\end{array}$ \\
\hline$u_{r m s-n o r m}$ & 1.91 & - & - & $0.16(8.4 \%)$ \\
\hline$u_{r m s}(\mathrm{~m} / \mathrm{s})$ & 3.19 & 0.12 & - & 0.12 \\
\hline$u_{\text {in }}(\mathrm{m} / \mathrm{s})$ & 1.67 & 0.13 & 0.017 & 0.13 \\
\hline
\end{tabular}

Low Value of $u_{\text {rms-norm }}$

Value Location: Plane $1 \mathrm{p}(0.500,0.236,0.081)$

\begin{tabular}{|c|c|c|c|c|}
\hline Variable & Value & $\begin{array}{c}\text { Precision } \\
\text { Uncertainty }\end{array}$ & $\begin{array}{c}\text { Bias } \\
\text { Uncertainty }\end{array}$ & $\begin{array}{c}\text { Total } \\
\text { Uncertainty }\end{array}$ \\
\hline$u_{r m s-n o r m}$ & 0.11 & - & - & $0.070(61.7 \%)$ \\
\hline$u_{r m s}(\mathrm{~m} / \mathrm{s})$ & 0.19 & 0.12 & - & 0.12 \\
\hline$u_{\text {in }}(\mathrm{m} / \mathrm{s})$ & 1.67 & 0.13 & 0.017 & 0.13 \\
\hline
\end{tabular}


Normalized Pitchwise RMS Levels, $v_{r m s-n o r m}$, for Three-Component LDV Measurements (Non-Orthogonal and Tilt Transformations)

Equations:

$$
\begin{gathered}
v_{r m s-n o r m}=\frac{v_{r m s}}{u_{i n}} \\
\frac{\partial v_{r m s-n o r m}}{\partial v_{r m s}}=\frac{1}{u_{i n}} \\
\frac{\partial v_{r m s-n o r m}}{\partial u_{\text {in }}}=\frac{-v_{r m s}}{u_{i n}^{2}} \\
U_{v_{\text {rms }- \text { norm }}}=\sqrt{\left(\frac{\partial v_{r m s-n o r m}}{\partial v_{r m s}} \cdot U_{v_{r m s}}\right)^{2}+\left(\frac{\partial v_{r m s-n o r m}}{\partial u_{i n}} \cdot U_{u_{i n}}\right)^{2}}
\end{gathered}
$$

High Value of $v_{\text {rms-norm }}$

Value Location: Plane $1 \mathrm{p}(0.500,-0.438,0.131)$

\begin{tabular}{|c|c|c|c|c|}
\hline Variable & Value & $\begin{array}{c}\text { Precision } \\
\text { Uncertainty }\end{array}$ & $\begin{array}{c}\text { Bias } \\
\text { Uncertainty }\end{array}$ & $\begin{array}{c}\text { Total } \\
\text { Uncertainty }\end{array}$ \\
\hline$v_{\text {rms-norm }}$ & 3.02 & - & - & $0.24(7.9 \%)$ \\
\hline$v_{\text {rms }}(\mathrm{m} / \mathrm{s})$ & 5.05 & 0.12 & - & 0.12 \\
\hline$u_{\text {in }}(\mathrm{m} / \mathrm{s})$ & 1.67 & 0.13 & 0.017 & 0.13 \\
\hline
\end{tabular}

Low Value of $v_{\text {rms-norm }}$

Value Location: Plane $1 p(0.500,-0.313,0.0)$

\begin{tabular}{|c|c|c|c|c|}
\hline Variable & Value & $\begin{array}{c}\text { Precision } \\
\text { Uncertainty }\end{array}$ & $\begin{array}{c}\text { Bias } \\
\text { Uncertainty }\end{array}$ & $\begin{array}{c}\text { Total } \\
\text { Uncertainty }\end{array}$ \\
\hline$v_{\text {rms-norm }}$ & 0.18 & - & - & $0.071(38.8 \%)$ \\
\hline$v_{\text {rms }}(\mathrm{m} / \mathrm{s})$ & 0.30 & 0.12 & - & 0.12 \\
\hline$u_{\text {in }}(\mathrm{m} / \mathrm{s})$ & 1.67 & 0.13 & 0.017 & 0.13 \\
\hline
\end{tabular}


Normalized Spanwise RMS Levels, $w_{r m s-n o r m}$, for Three-Component LDV Measurements (Non-Orthogonal and Tilt Transformations)

Equations:

$$
\begin{gathered}
w_{r m s-n o r m}=\frac{w_{r m s}}{u_{i n}} \\
\frac{\partial w_{r m s-n o r m}}{\partial w_{r m s}}=\frac{1}{u_{i n}} \\
\frac{\partial w_{r m s-n o r m}}{\partial u_{i n}}=\frac{-w_{r m s}}{u_{i n}^{2}} \\
U_{w_{r m s-n o r m}}=\sqrt{\left(\frac{\partial w_{r m s-n o r m}}{\partial w_{r m s}} \cdot U_{w_{r m s}}\right)^{2}+\left(\frac{\partial w_{r m s-n o r m}}{\partial u_{i n}} \cdot U_{u_{i n}}\right)^{2}}
\end{gathered}
$$

High Value of $w_{\text {rms-norm }}$

Value Location: Plane $1 p(0.500,0.0,0.485)$

\begin{tabular}{|c|c|c|c|c|}
\hline Variable & Value & $\begin{array}{c}\text { Precision } \\
\text { Uncertainty }\end{array}$ & $\begin{array}{c}\text { Bias } \\
\text { Uncertainty }\end{array}$ & $\begin{array}{c}\text { Total } \\
\text { Uncertainty }\end{array}$ \\
\hline$w_{\text {rms-norm }}$ & 2.44 & - & - & $0.20(8.1 \%)$ \\
\hline$w_{\text {rms }}(\mathrm{m} / \mathrm{s})$ & 4.07 & 0.12 & - & 0.12 \\
\hline$u_{\text {in }}(\mathrm{m} / \mathrm{s})$ & 1.67 & 0.13 & 0.017 & 0.13 \\
\hline
\end{tabular}

Low Value of $w_{\text {rms-norm }}$

Value Location: Plane $1 p(0.500,-0.313,0.081)$

\begin{tabular}{|c|c|c|c|c|}
\hline Variable & Value & $\begin{array}{c}\text { Precision } \\
\text { Uncertainty }\end{array}$ & $\begin{array}{c}\text { Bias } \\
\text { Uncertainty }\end{array}$ & $\begin{array}{c}\text { Total } \\
\text { Uncertainty }\end{array}$ \\
\hline$w_{\text {rms-norm }}$ & 0.13 & - & - & $0.072(55.3 \%)$ \\
\hline$w_{\text {rms }}(\mathrm{m} / \mathrm{s})$ & 0.22 & 0.12 & - & 0.12 \\
\hline$u_{\text {in }}(\mathrm{m} / \mathrm{s})$ & 1.67 & 0.13 & 0.017 & 0.13 \\
\hline
\end{tabular}




\section{Vita}

\section{Sachin Suresh Vakil}

Sachin Suresh Vakil was born in Carbondale, IL, on July 7, 1978. As a young child he lived in Ohio and Massachusetts before finally settling in Reston, VA, in April of 1984. He earned his Bachelor of Science in Mechanical Engineering from the University of Virginia, where he also completed one year of graduate school. In June of 2001, he transferred to Virginia Tech to finish his Master's degree. Working under his

advisor, Dr. Karen Thole, in the VTExCCL lab, Sachin finished his Master of Science in Mechanical Engineering late in November of 2002. 